\title{
VELOCITY ANALYSIS BASED ON MINIMUM ENTROPY
}

Diemer de Vries 


\section{VELOCITY ANALYSIS BASED ON MINIMUM ENTROPY}
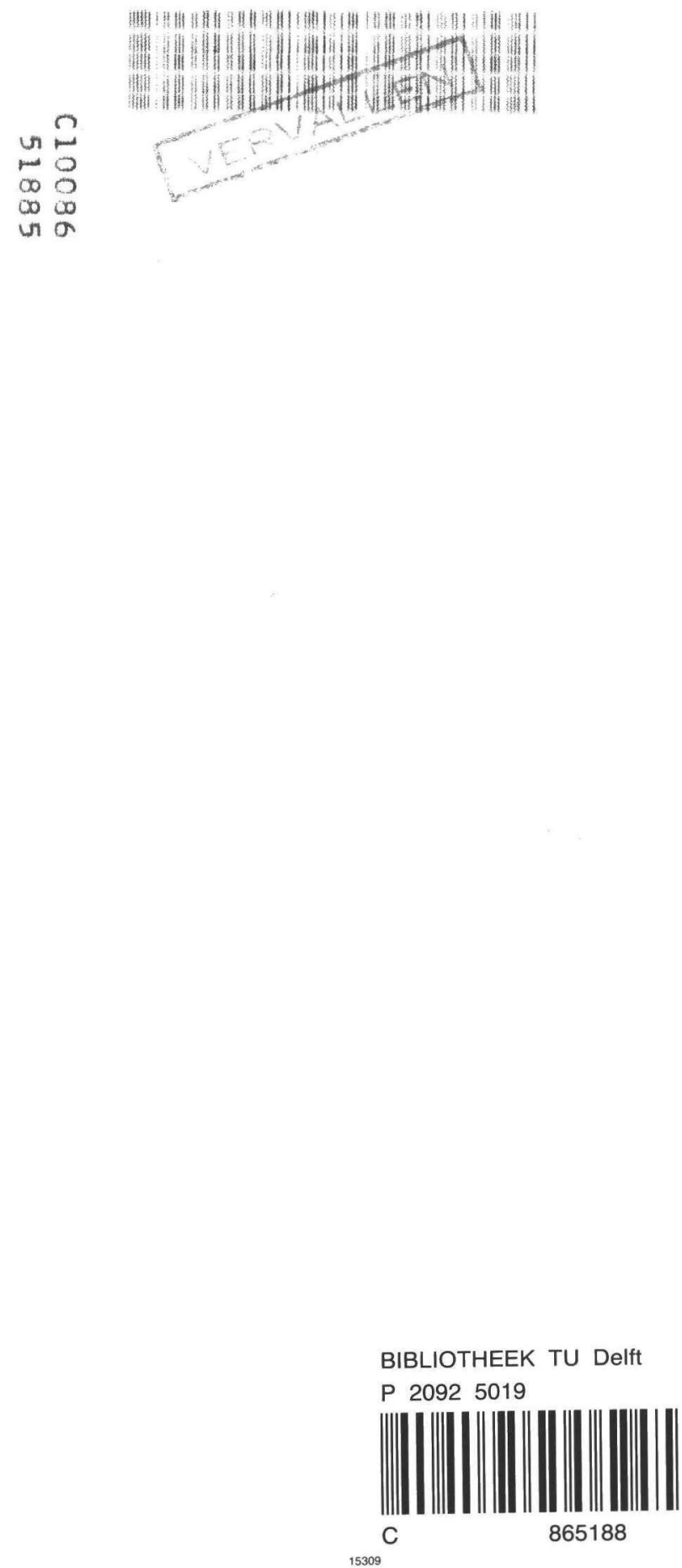


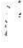




\title{
VELOCITY ANALYSIS BASED ON MINIMUM ENTROPY
}

\author{
PROEFSCHRIFT ter verkrijging van \\ de graad van doctor in de \\ technische wetenschappen \\ aan de Technische Hogeschool Delft, \\ op gezag van de Rector Magnificus, \\ prof.ir. B.P.Th. Veltman, \\ in het openbaar te \\ verdedigen ten overstaan \\ van het College van Dekanen op \\ donderdag 7 juni 1984 \\ te 16.00 uur door

\section{DIEMER DE VRIES} \\ natuurkundig ingenieur \\ geboren te Munnekeburen
}

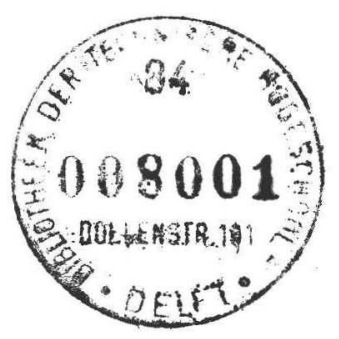

Gebotekst Zoetermeer/1984 
Dit proefschrift is goedgekeurd door de promotor prof.dr.ir. A.J. Berkhout 
Voor Nel, Tjalling en

het nog ongeboren tweede kind 



\section{PREFACE}

Within the group of Acoustics of the Delft University of Technology, development of echo-acoustical processing techniques has become the most important topic on the research program. In echo-acoustical research, velocity analysis requires ample attention, since appropriate processing of echo-acoustical data is possible only if the velocity distribution of the medium through which the acoustic waves have propagated is accurately known. In addition, velocity may provide valuable information on the medium properties. Operational velocity analysis techniques as described in the literature apply to reflection energy only; the velocity information available in diffraction energy is not used. Therefore, there exists a great need for velocity analysis techniques which are a1so applicable to data with an abundant amount of diffraction energy.

The idea that lateral dispersion of inverted data, as quantified by minimum entropy norms, could be a suitable criterion for velocity analysis of diffraction data was born in Delft a few years ago. In the present thesis, the evaluation of this idea is discussed, resulting in an operational technique called MEVA: Minimum Entropy Velocity Analysis.

I fee1 very privileged that I had the opportunity to do the research for my thesis in a dynamic team like the Delft group of Acoustics. I wish to express my special gratitude to the leader of that group, my promotor, professor A.J. Berkhout. I am afraid that, without his permanent critical, stimulating and constructive attention - which only seldom drove me to despair - this thesis would never have been completed.

I express my thanks also to my colleagues and some cooperating students, for being prepared to discuss my ideas and problems, for providing data sets suitable to test my theories and techniques, for giving me the opportunity to use their hardware and software, and to generate some illustrative figures. 
VIII

A special word of gratitude deserves our secretary Hanneke Mulder, who not only gave me a lot of mental support, but also typed out my preliminary manuscripts, so that I could read what I really had written down.

I a1so thank $\mathrm{mr}$. De Knegt of the drawing-office and $\mathrm{mr}$. Suiters of the photography service of our department, and mrs. Gerda Boone for their contributions to the final design of this book.

That MEVA will find useful application in echo-acoustical practice is my sincere wish.

Diemer de Vries

June 1984 


\section{CONTENTS}

VELOCITY ANALYSIS - IMPORTANCE, POSSIBILITIES AND

\section{LIMITATIONS}

I.1

INTRODUCTION

I. 2

IMPORTANCE OF VELOCITY ANALYSIS . . . . . . . . . . . . . 2

I.3

SUMMARY OF APPROACHES TO VELOCITY ANALYSIS . • . • . . . . . 6

I. 4 SURVEY OF VELOCITY ANALYSIS TECHNIQUES • • • • • • • • • • 11

I.4.1

General remarks . . . . . . . . . . . . . . . 11

I.4.2

Velocity analysis techniques concerning reflection energy • 11

$1.4 \cdot 3$

Velocity analysis techniques concerning refraction energy . 15

I. 4.4

I. 5

Velocity analysis techniques concerning diffraction energy 16

I.6 BASIC ELEMENTS OF MINIMUM ENTROPY VELOCITY ANALYSIS • • • • 17 OUTLINE OF THE FOLLOWING CHAPTERS . . . . . . . . . • . 18

II.1 INTRODUCTION • • . . . . • . . . . . . . . . . . . 21

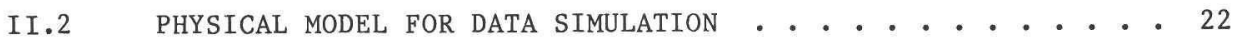

II.2.1 General physical model . . . . . . . . . . . . . . 22

II.2.2 Physical model for simulation of pressure-to-pressure primaries . . . . . . . . . . . . . . . 24

II.3 MODELING AND INVERSION IN ZERO-OFFSET TECHNIQUES • • • • • 34

II.3.1 Adaption of the physical primary model to the zero-offset configuration . . . . . . . . . . . . . . 34

II.3.2 Inversion scheme for zero-offset data . . . . . . . . . 37

II.4 EXAMPLES •. . . . . . . . . . . . . . . . . . 43

III INFLUENCE OF VELOCITY ERRORS ON ACOUSTIC INVERSION . . . 47

III.1 INTRODUCTION • • • • • • • • • • • • • • • • • • • • 47

III.2 INFLUENCE OF VELOCITY ERRORS ON THE SPATIAL WAVELET RESULTING AFTER MATCHED FILTERING . . . . . . . . . . . . . . 47

III.3 INFLUENCE OF VELOCITY ERRORS ON THE IMAGED RESULT IN THE SPACE-TIME DOMAIN . . . . . . . . . . . . . . . . 52 
IV

ENTROPY ................. . . 63

IV.1 INTRODUCTION .................. 63

IV.2 SHANNON'S ENTROPY AND RELATED NORMS . . . . . . . . 64

IV.2.1 Historical notes . . . . . . . . . . 64

IV.2.2 Shannon's entropy: a measure of a priori uncertainty . . 65

IV.2.3 A measure of a priori certainty . . . . . . . 67

IV.2.4 Entropy of an amplitude distribution: a measure of resolving power................... 69

IV.2.5 Entropy of an amplitude distribution related to uncertainty 72

IV.3 MINIMUM ENTROPY NORMS . . . . . . . . . . . 72

IV.3.1 Influence of amplitude parameter $a_{i} . . . . . . . .72$

IV.3.2 Generalization of the minimum entropy norm concepts . . 75

IV.3.3 Minimum entropy norms in literature . . . . . . . 81

IV.4 SENSITIVITY OF MINIMUM ENTROPY NORMS TO VARIATIONS IN

ELEMENTARY DATA TRACE PROPERTIES . . . . . . . . . 84

IV.5 MAXIMUM ENTROPY SPECTRAL ANALYSIS . . . . . . . . . 91

IV.6 CONCLUSIONS ................... . . 91

V MINIMUM ENTROPY IN RELATION TO VELOCITY ANALYSIS . . . . 93

V.1 INTRODUCTION . . . . . . . . . . . . . . . 93

V.2 APPLICABILITY OF MINIMUM ENTROPY NORMS IN SPACE-FREQUENCY

DOMAIN . . . . . . . . . . . . . . . 93

V.3 MINIMUM ENTROPY VELOCITY ANALYSIS .......... 109

V.3.1 Velocity analysis using minimum entropy norms in the spacefrequency domain ............. 109

V.3.2 Velocity analysis using minimum entropy norms in the space-

time domain .................. 115

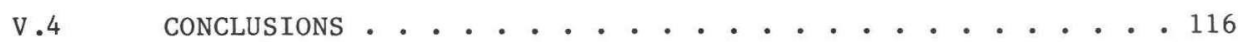

VI APPLICATIONS . . . . . . . . . . . . . 117

VI.1 INTRODUCTION ............................... 117

VI.2 SIMULATED ZERO-OFFSET DATA FROM DIFFRACTOR DISTRIBUTIONS • 117

VI.2.1 Distributions of elementary diffractors . . . . . . 117

VI.2.2 Diffracting discontinuities on reflectors . . . . . . 124

VI.3 SEISMIC APPLICATIONS . . . . . . . . . 127

VI.3.1 Simulated CMP data from one horizontal boundary . . . . 127

VI.3.2 Simulated CMP data from a model of three dipping layers . . 129

VI.3.3 Stacked field data . . . . . . . . . . 134 
VI.4 MEDICAL APPLICATIONS ....................... 137

VI.4.1 Zero-offset data measured on a phantom . . . . . 137

VI.4.2 Zero-offset data measured on real tissue . . . . . 138

VI.5 PRACTICAL RECOMMENDATIONS FOR MEVA APPLICATION $\ldots . \cdots 138$

\section{APPENDIX A}

A NOTE OF THE EFFECT OF VELOCITY ERRORS IN COMPUTERIZED

ACOUSTIC FOCUSING TECHNIQUES . . . . . . . . . . 141

APPENDIX B

MAXIMUM ENTROPY SPECTRAL ANALYSIS . . . . . . . . 145

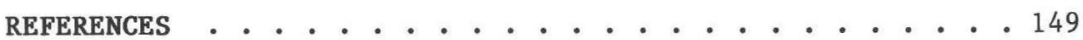

SUMMARY . . . . . . . . . . . . 157

SAMENVATTING ............................ 161

CURRICULUM VITAE . . . . . . . . . . . . . 165 



\section{CHAPTER I:}

\section{VELOCITY ANALYSIS - IMPORTANCE, POSSIBILITIES AND LIMITATIONS}

\section{I.1 INTRODUCTION}

The objective of this thesis is the development of a new velocity analysis technique which makes use of the spatial dispersion of acoustic wave fields. The technique can be employed in all areas where acoustic imaging is applied: exploration seismology, (sub)bottom profiling, medical diagnostics, nondestructive material testing.

In the above applications, the importance of velocity analysis appears in several ways:

(1) accurate knowledge of velocity information is required to optimize wave field extrapolation results as used in spatial inversion techniques,

(2) knowledge of propagation velocity provides extra information on characteristic properties of a medium (e.g. lithology, state of human tissue, composition and condition of materials). More specifically in seismic processing, velocity information is also indispensable for the data reduction procedure known as 'stacking'.

During the past decades, many papers on velocity analysis have been published. It appears that operational velocity analysis techniques apply to $\mathrm{r}$ e $\mathrm{f} 1 \mathrm{e}$ c$t i o n$ data as generated by continuous boundaries. In this context, diffraction energy as generated by discontinuities along a boundary is treated as detrimental information which should be suppressed. This the more remarkable, since diffraction data contain as much velocity information as reflection 
data. In this thesis, a velocity analysis technique is developed which aims at extracting velocity information from $d i f f r a t a$, thus being applicable to configurations as complex geologic structures, human tissues, inhomogeneous materials, etc. On the other hand, the proposed technique may be applied to reflection data as well, thus forming an interesting alternative to existing methods.

In this introductory chapter, firstly the importance of velocity analysis, as indicated above, will be considered in more detail. Next, different approaches with their advantages and limitations are discussed, followed by a survey of available techniques. Then, the basic ideas behind the velocity analysis technique developed in this thesis are summarized. Finally, an outline of the other chapters is given.

\section{I.2 IMPORTANCE OF VELOCITY ANALYSIS}

The essential role of propagation velocity in acoustic imaging is most simply shown as follows. Echo data are recorded as a function of $t i m$. After spatial $i \mathrm{n} v$ e $\mathrm{s} i \circ \mathrm{n}$, the result represents the acoustic image of the medium as a function of $d$ e $p t h$. Obviously, correct 'mapping' of time into depth is determined by the $v$ e 1 o c $i$ t $y$ distribution of the medium. Hence, without accurate knowledge of this velocity distribution a reliable image can never be obtained. When this knowledge is not available a priori, the crucial problem to be solved is how to extract velocity information from the recorded data before, or incorporated in, the inversion process.

The essence of spatial inversion is that, for different positions of source(s) and detector(s), the recorded reflection and diffraction data are d o w n $\mathrm{w}$ a r d e $\mathrm{x} t \mathrm{r}$ a p o 1 a $\mathrm{t}$ e $\mathrm{d}$ to the discontinuities in the medium from which they originate. In computerized inversion techniques, downward extrapolation is performed using an inversion operator in which propagation velocity plays a vital role. After downward extrapolation to a certain depth 1 evel $\mathrm{z}_{\mathrm{m}}$, an estimation of the reflectivity at depth $\mathrm{z}_{\mathrm{m}}$ is obtained by $\mathrm{i} \mathrm{m}$ a $\mathrm{g} i \mathrm{n} g$, i.e. by selecting the data in a time window around $t=0$. By repeating this procedure for all depth levels of interest $\left(z_{m}=z_{1}, z_{2}, \ldots, z_{M}\right)$, an acoustic image of the medium is formed. The combined process of inversion 
(downward extrapolation) and imaging is in seismic nomenclature denoted as m i g r a $t$ i o n.

It should be noted that in real-time acoustic imaging - often applied in medical diagnostics and then better known as acoustic fo $c$ u s $s$ in $g$ the above concept also holds: focussing is an approximation of wave field inversion. For a well-resolved and correctly positioned focus point it is necessary that a good estimate of the medium velocity or velocity distribution is used.

Fig. I-1 shows the effect of a velocity error on the image of a finite, tilted reflector with a central opening ('reflectivity drop'). It is seen in Figs. I-1c,e that a velocity error causes a $p$ o s i t $i$ o $\mathrm{i} n \mathrm{~g}$ error as well as deterioration of 1 a $t$ e $r$ a 1 r e s o 1 u $t i \circ n$ : the edges of the reflector image are not well defined, i.e. the image becomes more d i s p e r s e d.

Besides being a parameter of high importance in the inversion process, propagation velocity is a characteristic material constant. Hence, accurate knowledge of velocities can be used for $c h$ a $r a t e r i z a t i o n$ of materials. In tabel I/1, the propagation velocities of pressure waves are given for several media as found in the three areas of application mentioned in the introduction: sediments (seismology), tissues (medical diagnostics) and construction materials (non-destructive testing).

It is seen that, for the human $t i s s u$ e $s$ considered (except fat), the velocity values are found within a small range around $1560 \mathrm{~m} / \mathrm{s}$. Hence, tissue characterization on velocity criteria is only possible if the velocity values can be accurately determined. For tissues in vivo, characterization is even more difficult, since propagation velocity varies during contraction (Mol, 1981).

Finally, we mention the necessity of velocity analysis for a specific $s$ e $i \mathrm{~s}$ m i c application. In conventional seismic processing, data traces for which source and detector positions have the same midpoint (Common Mid Point gather), are combined ('stacked') to one trace, i.e. enhancement of signal-to-noise ratio is obtained by $d$ a $t a r$ e d u c t $i$ o $n$. The principle is illustrated in Fig. I-2 for a horizontal reflector. Note that the CMP 

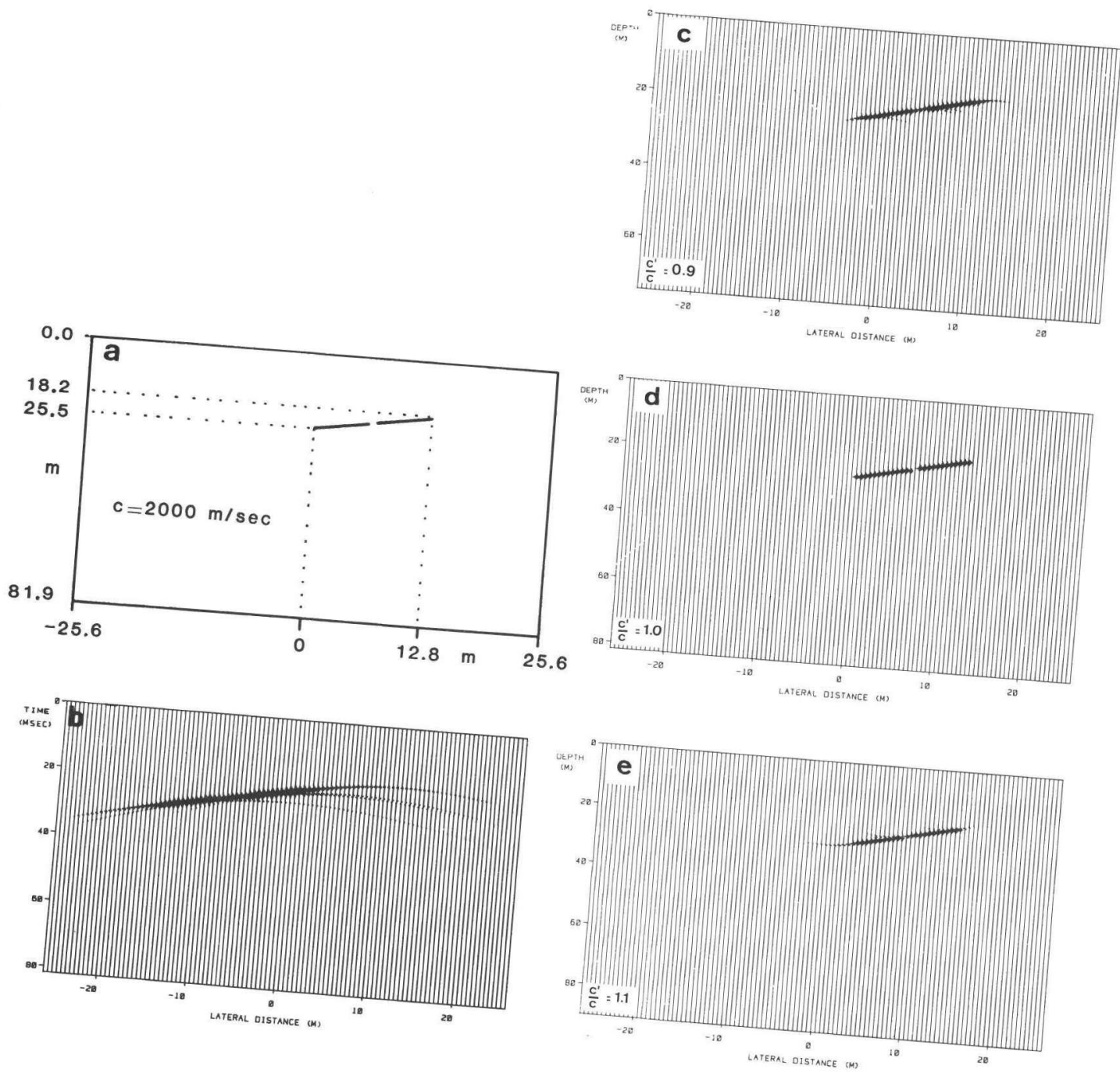
tilted reflector with a central 'reflectivity drop'.

b: zero-offset response

c: image after extrapolation with $-10 \%$ relative velocity error

e: id. with $+10 \%$ relative velocity error 
Table I/1

Some media and their propagation velocities for pressure waves.

\begin{tabular}{|l|l|l|}
\hline sediments & medium & velocity [m/s] \\
(seismology) & weathered layer & ca. 1000 \\
& unconsolidated & \\
& sands and clays & ca. 2000 \\
& consolidated & \\
& sands and clays & ca. 3000 \\
& carbonate rocks & ca. 4000 \\
\hline tissues & fat & $1445 \pm 35$ \\
(medical & spleen & $1555 \pm 35$ \\
diagnostics) & 1 iver & $1580 \pm 30$ \\
& kidney & $1563 \pm 5$ \\
& heart & ca. 1570 \\
& blood & ca. 1570 \\
& bone & ca. 3000 \\
\hline materials & concrete 4500 \\
(non-destructive & steel & ca. 5900 \\
testing) & perspex & ca. 2700 \\
\hline
\end{tabular}

gather forms a $\mathrm{h}$ y $\mathrm{p}$ e $\mathrm{r}$ b o $1 \mathrm{i}$ c reflection pattern (Fig. I-2b). It is seen that stacking is a two-step process: the CMP gather is lined up by 'normal moveout correction' (Fig. I-2c) and added to approximate a zero-offset trace at the gather midpoint (Fig. I-2d). For the simple case considered here, appropriate normal moveout correction is fully determined by the propagation velocity between surface and reflector. 


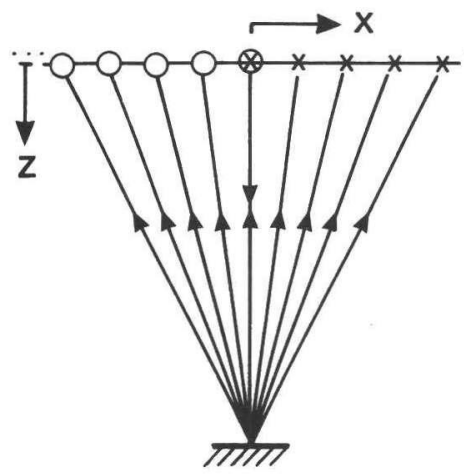

a

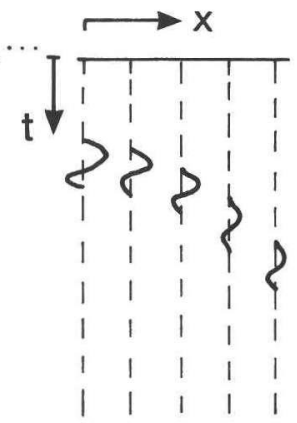

b

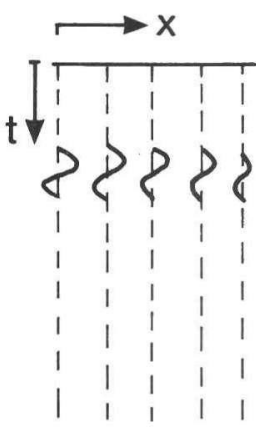

C

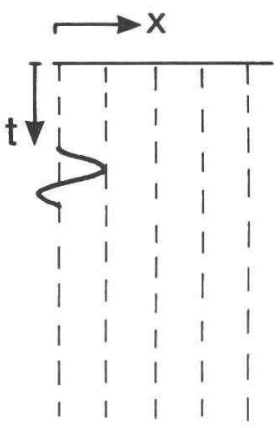

d

Figure I-2: Stacking of a common midpoint gather.

a: source-detector configuration and ray geometry for a horizontal reflector

$\mathrm{b}$ : common midpoint gather

c: common midpoint gather after normal moveout correction

d: post-stack trace, approximating a zero-offset trace at the midpoint

From the considerations in this section the conclusion can be drawn that accurate velocity analysis is very important in acoustic (especially seismic) imaging.

\section{I.3 SUMMARY OF APPROACHES TO VELOCITY ANALYSIS}

In order to explore the interior structure of a medium, the area of interest may be covered with a sufficiently dense grid of sources and detectors, and an aliasing-free c o m p l e $t$ e $d$ a $t$ a $s$ e $t$ may be recorded, i.e. for each source the response is monitored by a full range of detectors, see Fig. I-3a. Then, complete acoustic information about the medium will be obtained. 


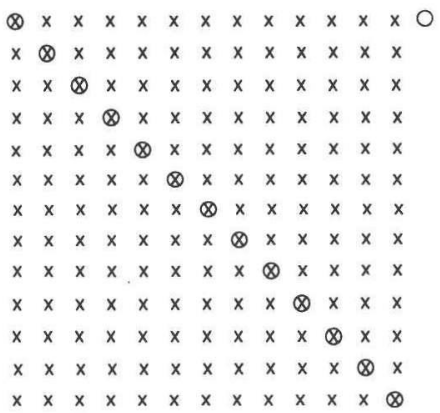

a

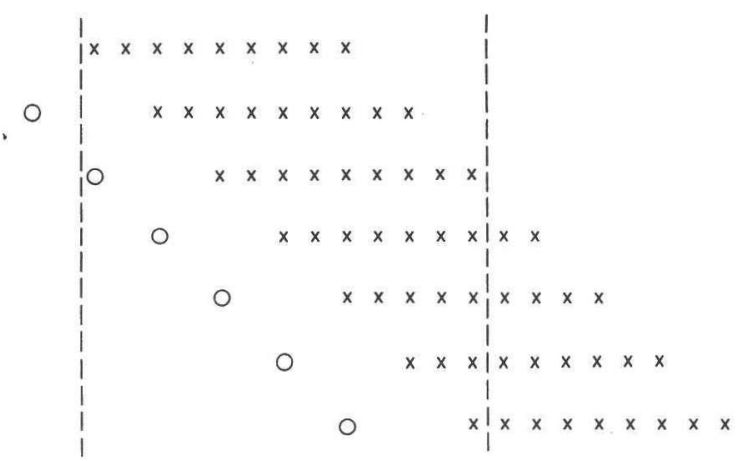

b

Figure I-3: Schematic configurations of sources $(0)$ and detectors $(x)$ to obtain

a: a complete data set

b: a multi-trace data set

Note that each row represents the geometric configuration for one physical experiment.

As will be argued more extensively in chapter II, a complete data set can elegantly be represented in the form of a data $m a t r i x$, see Fig. I-4.

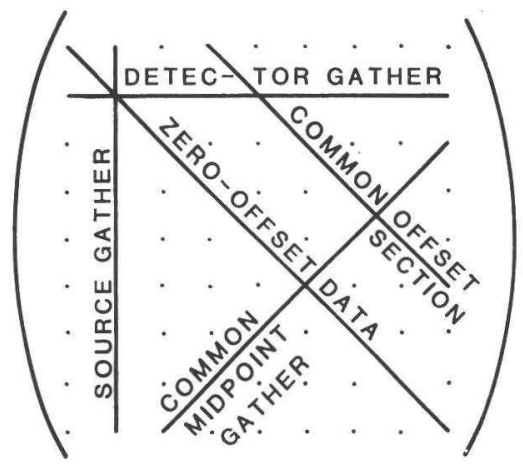

Figure I-4: Representation of a complete data set as a data matrix.

In this matrix, each column represents the data generated in one physical experiment, i.e. the responses of one source at the respective detectors. We denote such a column of the data matrix as a source gather ${ }^{1}$ ). Each row

1) In seismic nomenclature, the data from one physical experiment is called a seismic record. 
represents the response of all sources at a given detector, i.e. a detector gather. It is easily seen that common midpoint gathers as discussed in the previous section appear as antidiagonals in the data matrix. The zero-offset data form the main diagonal. The subdiagonals represent common offset sections, giving for each source the response at a detector on a given distance (offset).

A complete data set may include reflection as well as refraction and diffraction energy. Hence, spatial inversion of a complete data set without any data reduction ('pre-stack inversion') yields full acoustic information about the medium.

In spatial inversion of a complete data set, the data of each physical experiment, i.e. each column of the data matrix, is inverted. The result thus obtained is gathered according to the depth points of origin, thus forming Common Depth Point gathers. All CDP gathers are stacked and combined to form a true CDP-stacked section, representing the acoustic image of the medium considered. Performing this migration process, v e 1 o c $i$ t y information can be extracted in three different ways:

(1) CDP gathers have maximum lateral c o h e r e n c y if the correct velocity distribution of the medium has been used in the inversion process, (2) CDP-stacked traces have maximum a m p 1 i t u d e (of the envelope) if obtained with correct velocity,

(3) the complete CDP-stacked section (i.e. the pre-stack migration result) has minimum 1 a $t$ e $r$ a 1 d $s$ p $r s i o n$ if obtained with correct velocity. As shown in this thesis, lateral dispersion can be properly quanti-

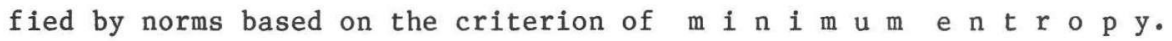

Inversion of complete data sets requires acquisition and processing of huge numbers of data. Hence, for practical and economic reasons, complete data sets are approximated by $\mathrm{m} \mathrm{u} 1 \mathrm{t} \mathrm{i}-\mathrm{t} \mathrm{r}$ a $\mathrm{c}$ e $\mathrm{d}$ a $\mathrm{t}$ a $\mathrm{s}$ e $\mathrm{t} s$ obtained with a sparsed source-detector configuration, an example of which is given in Fig. I-3b. Note that missing detectors correspond with zero's in the columns of the data matrix.

Traditionally in seismic applications, multi-trace data sets are arranged to c $\circ \mathrm{mm} \circ \mathrm{n} \mathrm{mid} \circ \mathrm{int}$ (CMP) $\mathrm{g}$ a $\mathrm{th}$ e $\mathrm{r}$ when used as input for velocity analysis procedures. This preference of CMP gathers has two basic reasons: 
(1) In many practical situations, reflection energy within one CMP gather is generated by a s m a 11 part of each boundary: see Fig. I-2a for the simple situation of a horizontal reflector. Hence, in many practical situations the boundaries may be considered as $f 1$ a $t$ within a CMP gather range.

(2) In many practical situations, reflection data from a flat boundary within one CMP gather form patterns which may be considered as $h$ y $p$ e b $\circ 1 \mathrm{i}$, see Fig. I-2b. The asymptotes of each hyperbola are determined by the structure and the $v$ e 1 o c $i t y$ distribution of the medium between surface and reflectivity boundary considered. Hence, velocity information can be obtained by processing simple hyperbolic responses.

Velocity information can be extracted from CMP gathers in several ways:

(1) after normal moveout correction (Fig. I-2c), a CMP gather has maximum c o h e r e $\mathrm{n}$ c $\mathrm{y}$ if the correction has been performed according to the correct hyperbola. Also, in this case,

(2) the stacked trace (Fig. I-2d) has maximum reflection a m p 1 i $t$ d e .

Moreover, it can be shown that an estimation of the stack can be obtained by $\mathrm{m} i \mathrm{~g} r \mathrm{t} i \mathrm{n} g$ the CMP gather. Hence, velocity information can be extracted from

(3) the maximum reflection a m p 1 i $t$ u d e of the migrated CMP gather, or, as proposed in this thesis,

(4) the lateral extension of the migrated CMP gather quantified by $m i n i-$ m u m e n $t r o$ p y norms.

In practical applications CMP velocity analysis is concentrated on reflection energy only. To cope with refraction data as well, special techniques are required, e.g. $\tau_{-p}$ mapping (see subsection I.4.3). Diffraction energy is actually detrimental, due to interference with the hyperbolic reflection patterns. In case of highly discontinuous complex media, echo data is measured in which diffraction energy predominates. Hence, for such media CMP velocity analysis is not a realistic proposition: other approaches are to be developed.

As mentioned in the introduction of this chapter, operational velocity analysis techniques extracting velocity information specifically from diffraction energy are scarcely indicated in literature. In this thesis such a technique is proposed. The basic idea behind this technique is that the 1 a $t$ e r a 1 
d $i s p e r s i \circ n$ of diffraction energy is $m i n i m$ a 1 if inverted with c o r r e c t ve 1 o c i t y, see also Fig. I-1. Lateral dispersion appears as a lateral extension effect which properly can be quantified by norms based on the criterion of $\mathrm{m} i \mathrm{n} i \mathrm{mu}$ e $\mathrm{n} \mathrm{t} \mathrm{r}$ o $\mathrm{p} \mathrm{y}$. Instead of CMP gathers, c o m m o $n$ of $\mathrm{f} s$ e $t$ (including zero-offset) data should be used for velocity analysis on diffraction data, i.e. the distance between sources and detectors should be constant (including zero). Note that, instead of zerooffset data, stacked data (see Fig. I-2d) can be used as well.

The above classification of velocity analysis approaches is summarized in table I-2. In the next section a survey is given how these principles are worked out in various velocity analysis techniques as proposed in the 1iterature.

Table I/2

Classification of different approaches to velocity analysis.

\begin{tabular}{|c|c|c|}
\hline data arrangement & $\begin{array}{l}\text { data to be } \\
\text { included in } \\
\text { velocity analysis }\end{array}$ & $\begin{array}{l}\text { velocity analysis } \\
\text { criteria }\end{array}$ \\
\hline $\begin{array}{l}\text { multi-record } \\
\text { data set }\end{array}$ & $\begin{array}{l}\text { reflection } \\
\text { refraction } \\
\text { diffraction }\end{array}$ & $\begin{array}{l}\text { * coherency of } \\
\text { CDP gather } \\
\text { *maximum amplitude of } \\
\text { stacked CDP gather } \\
\text { *minimum entropy of } \\
\text { CDP-stacked section }\end{array}$ \\
\hline CMP gathers & reflection & $\begin{array}{l}\text { * coherency of normal } \\
\text { moveout corrected } \\
\text { CMP gather } \\
\text { *maximum amplitude of } \\
\text { stacked CMP gather } \\
\text { *maximum amplitude or } \\
\text { minimum entropy of } \\
\text { migrated CMP gather }\end{array}$ \\
\hline $\begin{array}{l}\text { common offset } \\
\text { sections (inclu- } \\
\text { ding zero-offset } \\
\text { and stacked data) }\end{array}$ & diffraction & $\begin{array}{l}\text { *minimum entropy of } \\
\text { migrated common } \\
\text { offset section }\end{array}$ \\
\hline
\end{tabular}




\subsection{SURVEY OF VELOCITY ANALYSIS TECHNIQUES}

\subsubsection{General remarks}

In human tissues, the propagation velocities of pressure waves approximate the value $1560 \mathrm{~m} / \mathrm{s}$ within a few percents, as was seen in Table I/1. Hence, in imaging techniques employed in $m$ e $d i c$ a 1 diagnostics, this value is commonly used independently of the specific tissues considered. Due to the random and relatively small fluctuations in the actual velocity distribution, this simplified approach often yields imaged results of acceptable quality. In non-destructive testing of $\mathrm{m}$ a $t$ e $\mathrm{r} i \mathrm{a} 1 \mathrm{~s}$, the test specimen usually consists of a single bulk medium (e.g. concrete, steel), the velocity of which is known by good approximation.

In $\mathrm{s}$ e $\mathrm{i} \mathrm{s} \mathrm{m} i \mathrm{cs}$, however, the sediments of interest show large velocity variations $(500 \mathrm{~m} / \mathrm{s}<\mathrm{c}<5000 \mathrm{~m} / \mathrm{s})$ in both vertical and lateral directions. Therefore, accurate velocity analysis is a topic of increasing interest in this field and most operational velocity analysis techniques have their origin in geophysical research.

In this paragraph, we shall give a survey on velocity analysis techniques of contemporary interest, paying attention to underlying principles and applicability. For reasons of surveyability, we arrange the velocity analysis techniques in three categories, viz.:

(1) techniques using $\mathrm{r}$ e $\mathrm{f} l$ e $\mathrm{c} t \mathrm{i}$ o $\mathrm{n}$ energy as input data,

(2) techniques using $\mathrm{r}$ e f r a c t $i \circ \mathrm{n}$ energy as input data,

(3) techniques using $d i f f r a c t i o n$ energy as input data.

\section{I.4.2 Velocity analysis techniques concerning reflection energy}

Most velocity analysis techniques are based on processing of CMP $\mathrm{r}$ e $\mathrm{f} 1 \mathrm{e} \mathrm{c}-$ $t i \circ n$ data. As discussed in section I.3, such data form - at least approximately - hy p e r b 1 i c patterns in the two-dimensional space-time $(x, t)$ domain. The apex of each hyperbola is given by the zero-offset (midpoint) travel time to the boundary considered, whereas the asymptotes are described by

$$
\mathrm{x}= \pm \mathrm{v}_{\text {stack }} \mathrm{t}
$$


The value of velocity $\mathrm{v}_{\text {stack }}$ is determined by depths, shapes and velocities of a11 layers between surface and boundary. $v_{\text {stack }}$ is denoted as such, since stacking of CMP data yields optimal result if the normal moveout correction (see Fig. I-2c) is performed according to the hyperbola with correct apex and asymptotes, i.e. correct $\mathrm{v}_{\text {stack }}$. Thus, optimization of the moveout-corrected result in terms of $c \circ \mathrm{h}$ e e $\mathrm{n} \mathrm{y}$ or the stacked data in terms of a $\mathrm{m}-$ p 1 i t u d e forms a technique for velocity analysis.

The above velocity analysis technique has some limitations. As mentioned before, it only deals with reflection data as generated by approximately flat boundaries. Moreover, the CMP response is purely hyperbolic only for a homogeneous overburden. For an inhomogeneous overburden the responses are approximately hyperbolic for small offsets only. The approximation deteriorates with increasing dips and increasing curvatures. Due to these imperfections, the accuracy of the above velocity analysis technique decreases with depth.

Velocity analysis based on processing of reflection data has been applied long since. Duirbaum (1954) published the theoretical basis for the hyperbolic mode1. Dix (1955) indicates how, for a horizontally layered system, the interval velocities of the respective layers can be recursively calculated

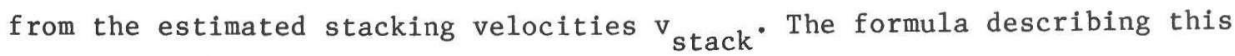
recursive relation has become a classic under the name 'Dix formula'. Taner and Koehler (1969) formulate coherency criteria to estimate $v_{\text {stack }}$ and they apply, with limited accuracy, the Dix formula also to dipping boundaries. Brown (1969) considers the influence of long-offset data. In the early seventies, much work has been done to extend the validity of the Dix model to dipping boundaries: Levin (1971), Larner and Rooney (1973), Shah (1973), Everett (1974). Krey (1976) generalizes, for small offsets, the theory of Dïrbaum to arbitrary dipping or curved boundaries in three dimensions and Hubral (1976) proves the applicability of this theory for practical situations. May and Straley (1979) refine the mathematical description of the deeper boundary responses, taking higher order terms into account. Berryhill (1979) applies wavefield 'datuming' (a kind of velocity replacement technique) to obtain better CMP results for the underlying strata. Khattri et al. (1980) propose the Fibonacci estimation technique as an alternative to coherency techniques. Hajnal and Sereda (1981) give an analysis of possible errors made in applying the Dix formula. Recently, extension of reflection data velocity 
analysis to media with lateral velocity variations has been discussed: Hubral (1980), Lynn and Claerbout (1982), Loinger (1983).

Since only reflection data obey the simple hyperbolic CMP model illustrated in Fig. I-2, the presence of $r$ e $f r$ a $c t i o n$ and particularly

d $i \mathrm{f} f \mathrm{r}$ a $\mathrm{c} t \mathrm{i}$ o $\mathrm{n}$ energy deteriorates the applicability of CMP velocity analysis techniques. This was also mentioned by Taner et a1. (1970) and later by Blackburn (1980). Arguing that downward wave field extrapolation focusses diffraction energy and thus diminishes its smearing effect, Doherty and Claerbout (1976) propose to $\mathrm{m} \mathrm{ig} \mathrm{r}$ a $t$ e the pre-stack multi-trace data with a roughly estimated velocity before picking CMP gathers and estimating $v_{\text {stack }}$ by application of the usual coherency techniques. A more thorough approach is to migrate the pre-stack data with different velocity values, estimating directly the migration velocity by applying coherency techniques to the migrated result. This procedure was proposed by Gardner et al. (1974) and Sattlegger (1975), and applied in practice by Dohr and Stiller (1975) and Sattlegger et al. (1976). Owusu et al. (1983) extend the application of this method to three-dimensional data using a fast migration algorithm.

As mentioned in section I.3, migration of a CMP gather yields an estimate of the stack to be obtained from that gather. From paraxial (i.e. small offset) CMP data an accurate stack estimate can be found even after downward extrapolation with an erroneous velocity: then, imaging must be performed using a time window not around $t_{i m}=0$ as usual, but around a time $t_{i m} \neq 0$. The stack is we11-resolved but positioned at an erroneous depth: velocity error and depth error are exchanged. The actual value of $t_{i m}$ depends on the velocity error, so that this value contains velocity information. This phenomenon was first described by Doherty and Claerbout (1974). Yilmaz and Chambers (1980) evaluated the possibility of velocity extraction, applying a special mapping procedure to determine $t_{i m}$ and using maximum amplitude as a criterion for optimal migration. In a paper on the effects of velocity errors on migration and focussing, De Vries and Berkhout (1984) place the exchangeability of velocity errors and depth errors, as present in recursive migration techniques, in a wave theoretical context. They also indicate the possibility to extract velocity information from the time shift denoted above as $t_{i m}$. 


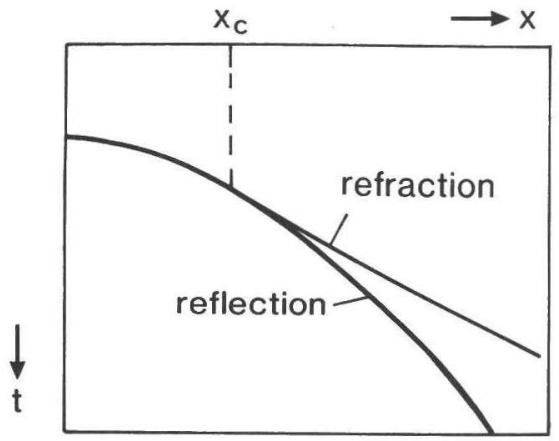

a

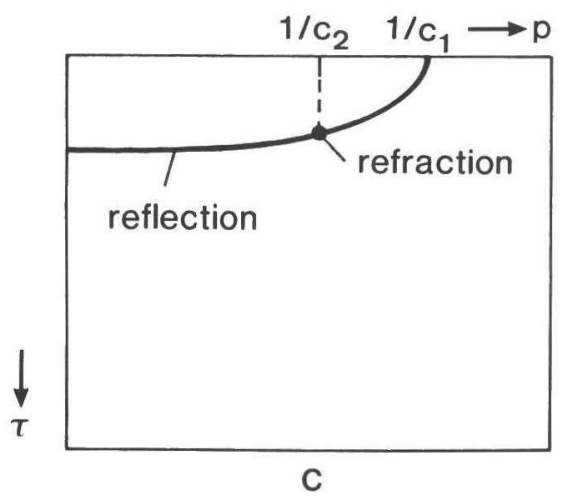

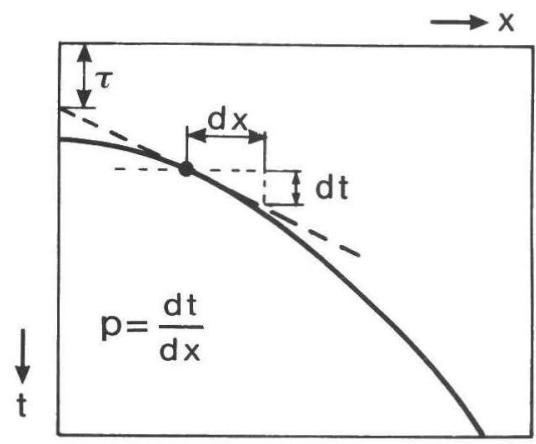

b

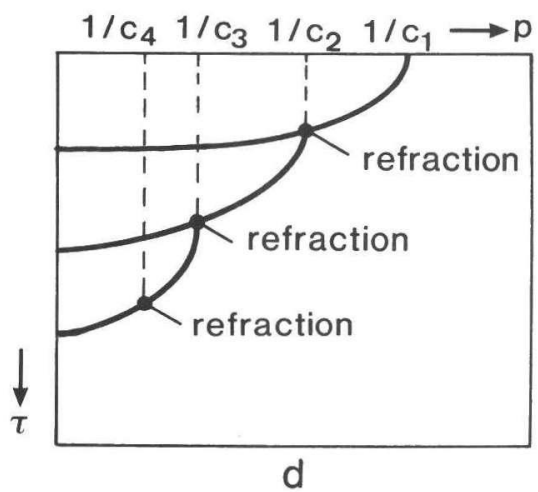

Figure I-5: Schematic illustration of $\tau-p$ mapping (slant stacking) of CMP reflection and refraction data

a: CMP gather on a horizontal boundary between two media with velocities $c_{1}$ and $c_{2}$ ( $x_{c}$ is half offset corresponding with critical angle)

$\mathrm{b}$ : to the definition of parameters $\mathrm{p}$ and $\tau$

c: CMP gather after $\tau-p$ mapping

d: CMP gather on a horizontal 3-boundary configuration after $\tau-p$ mapping 


\section{I.4.3 Velocity analysis techniques concerning refraction energy}

Compressional p-waves incident on an acoustic boundary under critical angle generate, besides a reflected wave, a $r$ e $\mathrm{f} \mathrm{r}$ a $\mathrm{c} t \mathrm{e} d$ wave. This refracted p-wave (in geophysics also called 'head wave') travels along the boundary with the velocity $c_{2}$ of the lower layer. Refraction energy is radiated to the sur-

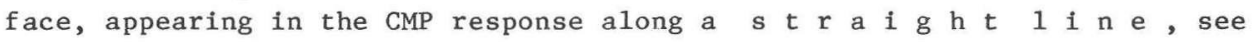
Fig. I-5a. By transforming the x-coordinate into horizontal 'slowness' parameter $\mathrm{p}$ and the $\mathrm{t}$-coordinate into intercept time $\tau$, see Fig. I-5b, the hyperbolic part of the CMP response is transformed into an e $11 \mathrm{ips}$ e and the linear part (representing refraction energy) into a 'focus' $p \circ i \mathrm{n}$ at $p=1 / c_{2}$, see Fig. I-5c. For a configuration of horizontal boundaries this process of ' $\tau-p$ mapping' results in a pattern of ellipse-like curves, the intersections of which denote refraction energy and are positioned at $\mathrm{p}=1 / \mathrm{c}_{\mathrm{n}+1}$, see Fig. I-5d. Hence, the positions of the refraction energy points after $\tau-p$ mapping yield velocity information.

As a logic continuation of the work by Doherty and Claerbout (1976), Schultz and Claerbout (1978) introduce the principles of the $\tau-p$ mapping procedure mentioned above, which they denote as $s 1$ a $\mathrm{t} s \mathrm{tack} i \mathrm{ng}$, since the transformation is carried out by summing the data along slanting straight lines (see also Fig. I-5b). They indicate how velocity analysis techniques using coherency criteria can be applied to both reflection and refraction data after slant stacking, also in media with lateral velocity variations. Diebold and Stoffa (1981) follow the same principles, whereas Stoffa, Diebold and Buhl (1982) explicitly include wide aperture data. Clayton and McMechan (1981) apply velocity analysis to slant stacked refraction data after migration, leaving reflection data out of consideration. In a quite different approach, Weglein et al. (1981) use slant stacked data including refractions as input for an algorithm inverting reflection coefficients to velocity data based on the Lippmann-Schwinger and Schroedinger equations. Schultz (1982) presents a method by which interval velocities are determined applying coherency techniques to recursively migrated slant stacked data. Aki and Richards (1980) indicate how in the $(\tau, p)$ domain velocity analysis can be applied to media with velocity gradients. Research on this topic is nowadays done in the group of acoustics of the Delft University of Technology.

As a final remark, it should be mentioned that a more extensive survey on velocity analysis techniques as applied in seismics is given by Hubral and Krey (1980). 


\section{I.4.4 Velocity analysis techniques concerning diffraction data}

As mentioned in the previous subsections, and more systematically analyzed by Taner et a1. (1970) and Blackburn (1980), CMP gathers loose their simple geometric properties when comprising an abundant amount of scattering $\mathrm{d}$ i f $f \mathrm{r}$ a $\mathrm{ct} i$ o $n$ energy. Hence, for complex, laterally discontinuous structures, velocity analysis techniques based on the hyperbolic CMP concept are not applicable.

As indicated in section I.3, two approaches may be adopted to perform velocity analysis in the presence of abundant diffraction data:

(1) pre-stack inversion of multi-trace data sets. We saw (subsection I.4.2) that this approach was discussed by Gardner et al. (1974), Sattlegger (1975), Doherty and Claerbout (1976), Owusu et a1. (1983). In the philosophy behind velocity analysis techniques as considered by the above authors diffraction energy is 'tolerated' as an inevitable part of the input data to be coped with, and certainly not as a source of velocity information.

(2) treatment of diffraction data as a s p e c i f i c source of velocity information. Then, recorded data should be gathered such that diffraction energy is clearly profiled. For this purpose, com mo $n$ of $\mathrm{f}$ e $t$

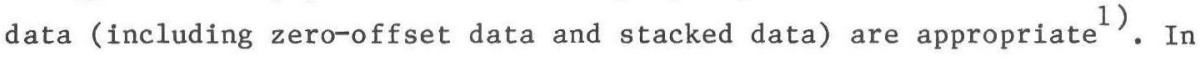
common offset data acquisition, the response of a single diffractor has a hyperbolic shape, as has the response of a reflector in CMP data acquisition.

Several authors have proposed ideas to tackle the problem of velocity analysis in complex media using the second approach outlined above. Since medical applications of echo-acoustics are usually performed with zero-offset scanning devices, it can be understood that researchers from this field have largely contributed to the development of diffraction data analysis.

1) This is confirmed by Hubral (1975), where he states: "Diffraction curves which are observed on stacked sections ... provide useful subsurface information about a diffractor in a similar way as CDP (= CMP, DdV) arriva1 times provide information about a reflector. They can therefore be considered for the purpose of interval velocity computations and time-to-depth conversions as we11". 
Sanzgiri (1977) discusses a zero-offset velocity analysis technique which seems applicable to flat reflectors only. Dameron $(1979,1980)$ describes a zero-offset velocity analysis technique applicable to media with weak velocity variations in one dimension. Much work on velocity analysis has been done at Denver University, U.S.A., resulting in a series of papers on zero-offset or common offset velocity inversion algorithms applicable to media where the velocity variations are small perturbations on a (preferably constant) reference value: Cohen and Bleistein (1979), Gray and Bleistein (1980), Gray et al. (1980), Gray (1981 a, b), Bleistein and Cohen (1982). Raz (1981 a,b) presents a general theory on velocity profile inversion, the practical applicability of which is not yet clear. Robinson et a1, (1982) use the cross-correlation between two ultrasound zero-offset scans acquired from the same target to determine an 'image shift' from which velocity information can be obtained. Worth mentioning are also the efforts to extend velocity analysis techniques as used in transmission tomography to reflection and diffraction data: Wade et a1. (1978), Kenue and Greenleaf (1982), Kaveh et al. (1982), Hiller and Ermert (1982). Until now, the results seem to be not practically applicable.

The conclusion must be drawn that a velocity analysis technique with general applicability to common offset (including zero-offset) diffraction data is not available. As a contribution to the fulfillment of this gap, the author proposes the 'minimum entropy' technique which is the subject of this thesis. The basic elements of this technique are summarized in the next section.

\section{I.5 BASIC ELEMENTS OF MINIMUM ENTROPY VELOCITY ANALYSIS}

As mentioned before and seen in Fig. I-lb, diffraction energy appears in zerooffset records as hyperbolic patterns. M $\mathrm{i} g \mathrm{r}$ a $\mathrm{t}$ o $\mathrm{n}$ of the data has a $f \circ c u s s i n g$ effect on the diffraction energy: migration with a we11chosen operator containing the right $v$ e 1 o c i t y value makes the diffraction patterns collapse (Fig. I- 1 c) thus assigning minimum 1 a $t$ e $r$ a 1 d $i s p e r s i o n$ to the data. Hence, the lateral extension of migrated zero-offset (and, similarly, common offset) diffraction patterns can be used as a criterion for velocity analysis.

It will be shown that in the space-frequency domain the lateral extension of migrated zero-offset (or common offset) diffraction patterns - representing the focussing quality of the inversion operator - can be described in terms of 
band-limited spatial wavelets having minimum d $i s$ e $r s i o n$ in case of correct migration velocity.

A suitable way of quantifying wavelet dispersion is the application of norms known in geophysical 1iterature as 'minimum entropy' norms. Therefore, we

shall refer to our technique as $\underline{M} \mathrm{i} n \mathrm{im} \mathrm{u} \underline{\mathrm{E}} \mathrm{n} \mathrm{t} \mathrm{r}$ o $\mathrm{p}$ y $\underline{\mathrm{V}} \mathrm{e}$ o c $i-$ $\mathrm{t} y$ A $\mathrm{n}$ a $1 \mathrm{y} \mathrm{s} \mathrm{i} \mathrm{s}$, abbreviated as MEVA.

Since the spatial wavelet concept describing inverted zero-offset diffraction data is also valid for inverted CMP reflection data, MEVA also applies to reflection data, forming an alternative to the techniques mentioned in subsection I.4.2.

The principles and preliminary results of MEVA were first published by De Vries and Berkhout (1982). Recently, Hanlan et al. (1983) presented a paper in which a velocity analysis technique is proposed extracting velocity information from migrated diffraction data by a statistical whitening process.

\section{I.6 OUTLINE OF THE FOLLOWING CHAPTERS}

In chapter II, a wave theoretical model for zero-offset data is presented. Inversion of zero-offset data is described using a spatial matched filter performing phase correction as well as amplitude weighting. The inverted result is presented in terms of band-1imited spatial wavelets determined by spatial amplitude and phase spectra.

In chapter III, the influence of velocity errors on inversion is discussed. Particularly, effects on the phase spectrum and, hence, on the dispersion of the spatial wavelets are considered.

In chapter IV, the concept of 'entropy' and especially 'minimum entropy' is considered. Minimum entropy norms are defined and interpreted as measures of resolving power of a data set, specified by dispersion and sparsity. Basic properties of minimum entropy norms are evaluated.

In chapter $V$, the applicability of minimum entropy norms to spatial wavelets is shown, making them a tool for velocity analysis (MEVA). Elementary applications are presented. 
In chapter VI, applications of MEVA are discussed. Simulated as well as measured data sets are considered, including zero-offset as well as CMP data from various application fields. MEVA is shown to be a powerful technique with a wide applicability. 


\section{CHAPTER II: \\ WAVE THEORETICAL APPROACH TO ACOUSTIC MODELING AND INVERSION}

\section{II.1. INTRODUCTION}

In any echo-acoustical technique, two basic processes are to be discerned, viz. the forward process and the inverse process.

In the $\mathrm{f}$ o $\mathrm{r}$ w a $\mathrm{r}$ d process, a sound wave is generated at the surface of the system to be investigated. This sound wave propagates downward through the underlying medium, is reflected and diffracted by inhomogeneities and layer boundaries, then propagates upward, and finally is recorded in some aperture area at the surface. The forward process is also called $\mathrm{m}$ o d e $1 \mathrm{i} \mathrm{n} g$ process.

In the $i \mathrm{n} v$ e $\mathrm{s}$ e process, an acoustic image of the medium below the aperture area is formed by appropriate processing of the recorded data. This processing is called image reconstruction, spatial inversion, focussing or, especially in seismics, $m i g r$ a $t$ i o $n$. Basically, all inverse techniques aim at elimination of the propagation effects introduced by the forward process, as well as the influences of source and detector characteristics.

Both forward and inverse process can be represented by physical models based on acoustic wave theory. Propagation is described by the scalar acoustic wave equation, reflection and diffraction by the boundary conditions imposed to that equation. Kirchhoff used the wave equation, together with Huygens's principle stating that any wave front may be considered as a configuration of secondary sources, to develop his theorem which enables forward extrapolation of a 
sound field on a closed surface to any point enclosed. Rayleigh modified this theorem for data on (infinite) plane surfaces. Based on this, forward propagation of sound waves through a medium can be described by extrapolation operators which are often called generalized Rayleigh-operators. Berkhout (1982) shows that, in general, these operators can be elegantly represented in matrix notation, and in special cases as a spatial convolution. On the other hand, the inverse process where propagation effects are elininated can be described by inverse extrapolation operators, derived from the forward operators by matrix inversion or deconvolution. In later chapters about velocity analysis (chps. V, VI), mainly $z$ e $r$ o - o f $\mathrm{f} s$ e $t$ data will be considered, i.e. data acquired by positioning sources and detectors at the same places in the aperture area. Therefore, in this chapter emphasis will be laid on the discussion of a model describing forward and inverse processing of zero-offset data. Since many elements of this derivation are clearly described by Berkhout (1982), references to this textbook are made where possible.

\section{II.2 PHYSICAL MODEL FOR DATA SIMULATION}

\section{II.2.1 General physical model}

In Fig. II-1, the solid lines denote a three-dimensional medium consisting of irregularly shaped layers below a horizontal surface plane on which a configuration of sound sources $(0)$ and detectors $(x)$ is arranged. In order to model the sound field at the detector positions, we cover the medium with a grid of horizontal planes in each point of which the reflection properties are given, depending generally on frequency and angle of incidence. For perfect modeling,

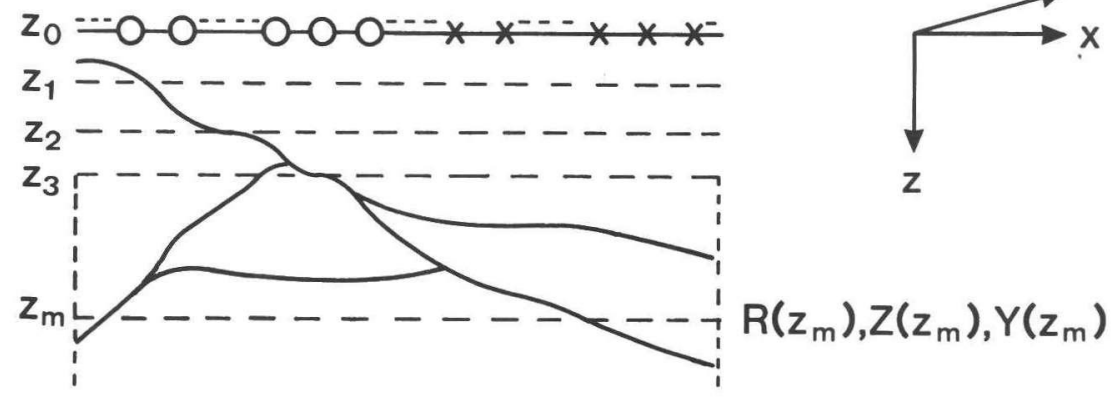

Figure II-1: General physical model for echo-acoustical data. 
the vertical distance between the horizontal planes should be infinitesimally small, but in practical situations a finite interval may be taken depending on the wavelengths of the acoustic waves as well as on the density and propagation velocity gradients in the medium considered. Between the horizontal planes, the propagation is described by the appropriate homogeneous extrapolation operator. There are three types of operators describing forward extrapolation of a sound field from a plane through a homogeneous medium to a point, denoted as WI, WII and WIII. Their applicability depends on the field parameters considered: operator WI describes extrapolation from normal particle velocity to pressure, WII describes extrapolation from pressure to pressure or from normal velocity to normal velocity, WIII describes extrapolation from pressure to normal velocity. The derivation of those operators from wave theory is described by Berkhout (1982, chp. 5).

When the medium consists of s o 1 i d materials, a compressional sound wave, often denoted as p-wave, may be converted into other wave types, such as shear waves, bending waves a.o.. Since a - certainly interesting - study of this wave conversion falls beyond the scope of this thesis, we consider in our model o n $1 \mathrm{y}$ t h e p - w a ve s treating the other wave types as noise, as is done in most studies on inversion. This means that, in the horizontal planes considered, only the reflection properties for $p$-waves have to be specified. The form in which those properties must be described depends on the source and detector types. If the source has a monopole character and hence defines normal particle velocity, whereas the detector has a dipole characteristic thus being pressure-sensitive, the reflection properties should be given in terms of local impedance $Z$. If source and detector are of the same type, the reflection properties must be specified in terms of reflectivity R. Finally, in case of a pressure source and a velocity detector, the local admittance $Y$ is the suitable parameter to describe reflection properties. Thus, the propagation between two planes can in all cases be formulated by the homogeneous operator denoted above as WII.

In our mode1, we shall assume pressure sources and pressure detectors only, so that reflection properties will always be described in terms of pressure $r$ e $f 1$ e c $t i v i t y$. This does not affect the general validity of our model, since the other cases can simply be transformed to the above one: a velocity source is dealt with by replacing in the first step $\left(z_{0} \rightarrow_{z_{1}}\right)$ operator WII by operator WI, a velocity detector by replacing in the last step $\left(z_{1} \rightarrow z_{0}\right)$ operator WII by operator WIII. See Berkhout (1982, pg. 162). 
Besides primary reflection, between aperture plane and boundaries multiple reflections will occur. In our model, we shall neglect such multiples for simplicity reasons. Berkhout (1982, sections $6.9,6.10,7.6$ ) indicates how, if desired, multiples can be taken into account in forward as well as in inverse processing by relatively simple algorithms.

Applying the limitations mentioned above, the one-way wave equation may be used leading to a $\mathrm{p} r \mathrm{i} \mathrm{m}$ a $\mathrm{r} y$ simulation model for $\mathrm{p}$-waves which we shall discuss in more detail in the next subsection.

\section{II.2.2 Physical model for simulation of pressure-to-pressure primaries}

In Fig. II-2 the data flow in modeling two-dimensional multi-record pressure primaries from one depth level $\mathrm{z}_{\mathrm{m}}$ is shown. Fig. II-2a shows the schematic geometry, whereas Fig. II-2b represents the physical model in the form of a block diagram.

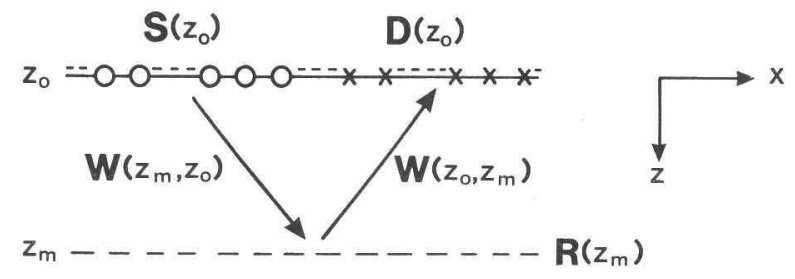

a

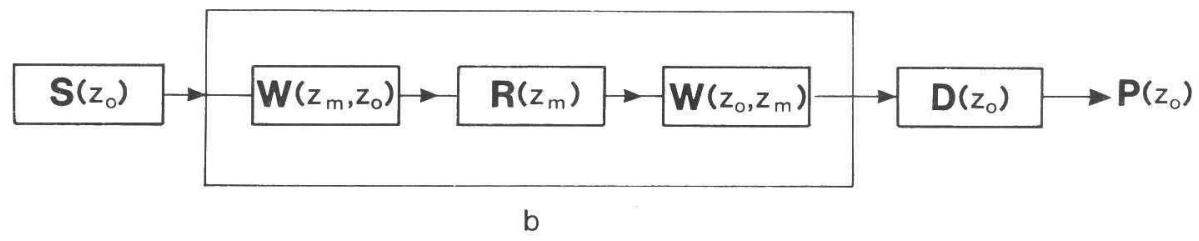

Figure II-2: Physical two-dimensional mode1 for simulation of pressure primary data from one depth level.

a. schematic geometry

b. block diagram

As Berkhout (1982, sections 6.2, 6.6) explains, the elements of the block scheme generally can be represented by m a $t \mathrm{r} i \mathrm{c}$ e so that the modeling 
process can be described by a series of matrix multiplications, summed over all depth layers of interest:

$$
\mathbf{P}\left(\mathrm{z}_{\mathrm{o}}\right)=\mathbf{D}\left(\mathrm{z}_{\mathrm{o}}\right)\left[\sum_{\mathrm{m}} \mathbf{W}\left(\mathrm{z}_{\mathrm{o}}, \mathrm{z}_{\mathrm{m}}\right) \mathbf{R}\left(\mathrm{z}_{\mathrm{m}}\right) \mathbf{W}\left(\mathrm{z}_{\mathrm{m}}, \mathrm{z}_{\mathrm{o}}\right)\right] \mathbf{S}\left(\mathrm{z}_{\mathrm{o}}\right) .
$$

Here $\mathbf{S}\left(\mathrm{z}_{0}\right)$ and $\mathbf{D}\left(\mathrm{z}_{0}\right)$ define the characteristics of sources and detectors respectively, $\mathbf{R}\left(\mathrm{z}_{\mathrm{m}}\right)$ describes the pressure reflectivity at depth level $\mathrm{z}_{\mathrm{m}}$, $W\left(z_{m}, z_{o}\right)$ and $W\left(z_{o}, z_{m}\right)$ the sound wave propagation from surface $z_{o}$ to depth level $z_{\mathrm{m}}$ and vice versa, whereas $\mathbf{P}\left(z_{0}\right)$ denotes the multi-record pressure recording at the surface.

The basic aims of forward and inverse processing can simply be indicated using matrix equation (II-1):

(1) in $\mathrm{f}$ o $\mathrm{r}$ w a $\mathrm{r} d$ processing, matrices $\mathbf{S}, \mathbf{D}, \mathbf{W}$ and $\mathbf{R}$ are known and primary pressure response $\mathbf{P}$ is calculated by a process of matrix multiplication,

(2) in $i \mathrm{n} v$ e $\mathrm{r}$ s e processing, $\mathbf{P}$ is recorded, $\mathbf{S}$ and $\mathbf{D}$ are supposed to be known, matrices $\mathbf{W}$ are (approximately) known and an estimation of $\mathbf{R}$ is found by a process of matrix inversion.

Next, we shall discuss the matrices composing Eq. (II-1) in some more detail. The elements of all matrices are described in the s p a c e - f r e q u e n c y domain.

(1) source matrix $\underline{\mathbf{S}}\left(\mathrm{z}_{0}\right)$

$\mathbf{S}\left(z_{0}\right)$ is the source matrix. Each c $\circ 1 \mathrm{u} m \mathrm{n}$ represents a source array used for one seismic experiment. Note that if elementary pressure sources (i.e. dipole sources) are used, each matrix column $\vec{S}_{n}\left(z_{0}\right)$ contains only one nonzero element the position of which indicates the position on the $x$-axis. Hence, written as a function in the space-frequency domain the $n^{\text {th }}$ column reads:

$$
S_{n}\left(x, z_{0}, w_{i}\right)=S\left(x, z_{0}, w_{i}\right) \delta(x-n \Delta x) .
$$

Such an elementary source can be considered as a spatial pulse at surface level $z_{0}$. In case of extended source arrays (length $2 K+1$ ), the position of the central non-zero column element indicates the array centre position on the $x$-axis: 


$$
S_{n}\left(x, z_{0}, w_{i}\right)=S\left(x, z_{0}, w_{i}\right) \sum_{i=-K}^{K} \delta(x-(n+i) \Delta x) .
$$

(2) downward propagation matrix $\underline{\mathbf{W}}\left(\mathrm{z}_{\mathrm{m}}, \mathrm{z}_{\mathrm{o}}\right)$

Primary energy propagation from surface level $z_{0}$ to depth 1 evel $z_{m}$ is described by matrix $\mathbf{W}\left(z_{m}, z_{0}\right)$ :

$$
\mathbf{S}\left(\mathrm{z}_{\mathrm{m}}\right)=\mathbf{W}\left(\mathrm{z}_{\mathrm{m}}, \mathrm{z}_{\mathrm{o}}\right) \mathbf{S}\left(\mathrm{z}_{\mathrm{o}}\right) \text {. }
$$

Let us consider one physical experiment, performed with a dipole source with unit strength - i.e. a spatial unit pulse - positioned at $\left(n \Delta x, z_{0}\right)$. Then, only the $n^{\text {th }}$ column vectors of $\mathbf{S}\left(z_{m}\right)$ and $\mathbf{S}\left(z_{0}\right)$ are of interest, $\vec{S}_{n}\left(z_{0}\right)$ having one non-zero element $\mathrm{S}_{\mathrm{nn}}\left(\mathrm{z}_{\mathrm{o}}\right)$ :

$$
\mathrm{S}_{\mathrm{nn}}\left(\mathrm{z}_{\mathrm{o}}\right)=\mathrm{S}_{\mathrm{n}}\left(\mathrm{n} \Delta \mathrm{x}, \mathrm{z}_{\mathrm{o}}, \mathrm{w}_{\mathrm{i}}\right)=1
$$

We now write:

$$
\vec{S}_{n}\left(z_{m}\right)=W\left(z_{m}, z_{o}\right) \vec{S}_{n}\left(z_{o}\right),
$$

or, considering $\mathrm{N}$ discrete data points at each depth level:

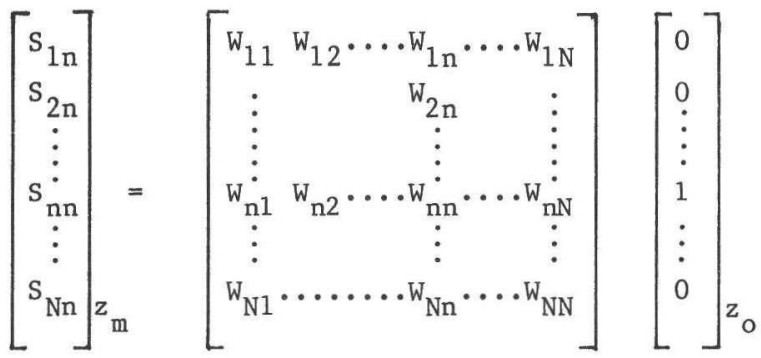

It is easily seen that $\vec{S}_{n}\left(z_{m}\right)$ as well as the $n^{\text {th }}$ c o 1 u $\mathrm{m}$ of matrix $\mathbf{W}\left(\mathrm{z}_{\mathrm{m}}, \mathrm{z}_{\mathrm{o}}\right)$ are 'spatial wavelets' which can be interpreted as the $\mathrm{s}$ p a t i a 1 i m p 1 s e $r$ e $p$ o $n$ s e to a source in point $\left(n \Delta x, z_{0}\right)$, see also Fig. II-3a.

In an analogous way it can be seen that the $n^{\text {th }} r$ o $w$ vector of matrix $\mathbf{W}\left(z_{\mathrm{m}}, \mathrm{z}_{\mathrm{o}}\right)$ represents the response in point $\left(\mathrm{n} \Delta \mathrm{x}, \mathrm{z}_{\mathrm{m}}\right)$ to a full array of dipole unit sources at surface level $z_{0}$, see Fig. II-3b. 


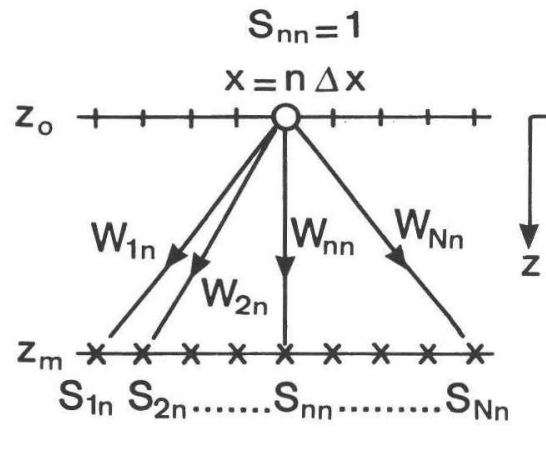

a

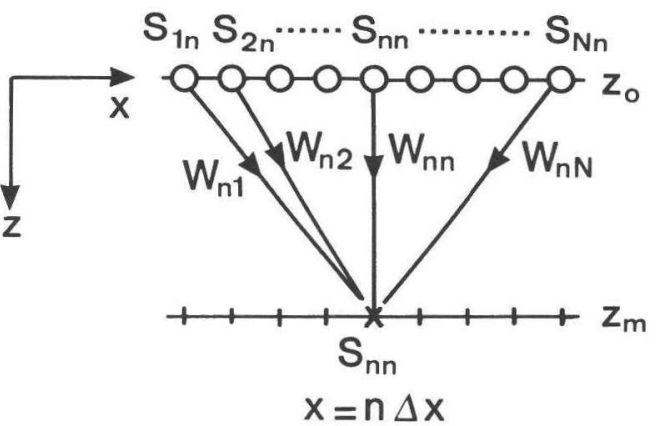

b

Figure II-3: Illustration to the interpretation of column vectors (a) and row vectors (b) of propagation matrix $\mathbf{W}\left(\mathrm{z}_{\mathrm{m}}, \mathrm{z}_{\mathrm{o}}\right)$.

For a homogeneous medium, the elements $\mathrm{W}_{\mathrm{pq}}$ of matrix $\mathbf{W}\left(\mathrm{z}_{\mathrm{m}}, \mathrm{z}_{\mathrm{o}}\right)$ are given by homogeneous extrapolation operator WII, multiplied with spatial increment $\Delta x$ to which we here assign unit value for simplicity. Berkhout (1982, section 5.6) shows that, for a homogeneous two-dimensional medium, operator WII and, hence, $\mathrm{W}_{\mathrm{pq}}$ is specified for $\mathrm{kr}_{\mathrm{pq}} \gg 1$ as:

$$
w_{p q}\left(x, z, w_{i}\right)=\Delta z_{m} \sqrt{\frac{j k}{2 \pi}} \frac{\exp \left(-j k r_{p q}\right)}{r_{p q} 3 / 2} \text {, }
$$

with:

$$
\begin{aligned}
\sqrt{j} & =\frac{1}{2} \sqrt{2}(1+j), \\
\Delta_{m} & =\left|z_{0}-z_{m}\right|, \\
k & =k(x, z)=\omega_{i} / c(x, z), \\
r_{p q}^{2} & =[(p-q) \Delta x]^{2}+\left(\Delta z_{m}\right)^{2},
\end{aligned}
$$

see Fig. II-4. Note that operator WII can be interpreted as the pressure field of a dipole source positioned at $\left(q \Delta x, z_{0}\right)$. 


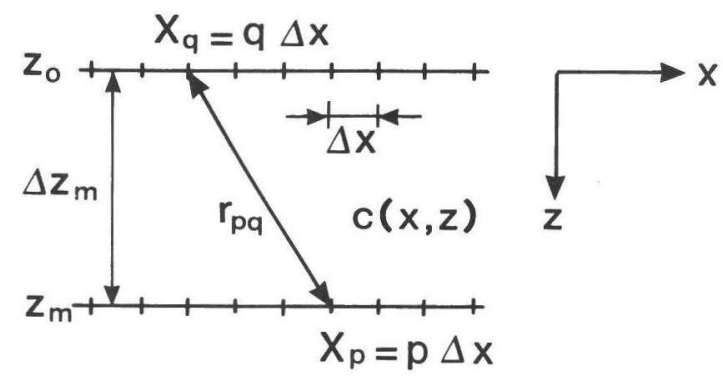

Figure II-4: Geometry to the two-dimensional homogeneous operator WII.

Due to the practical necessity of data truncation (aperture limitation), the elements of $\mathbf{W}\left(z_{\mathrm{m}}, z_{0}\right)$ are of interest only within a limited range of $r$. For example, the significant elements of the $n^{\text {th }}$ column vector, Fig. II-3a, lay within a limited range - say, $2 \mathrm{~L}-1$ points - around $\mathrm{x}=\mathrm{n} \Delta \mathrm{x}$. Then $\mathrm{k}(\mathrm{x}, \mathrm{z})$, Eq. ( $I-5 d$ ), is approximately determined by the velocity distribution around this point. Hence, matrix $W_{\left(z_{m}, z_{o}\right)}$ can be represented as a b a $n d$ matrix with a width of $2 \mathrm{~L}^{-1}$ points around the main diagonal:

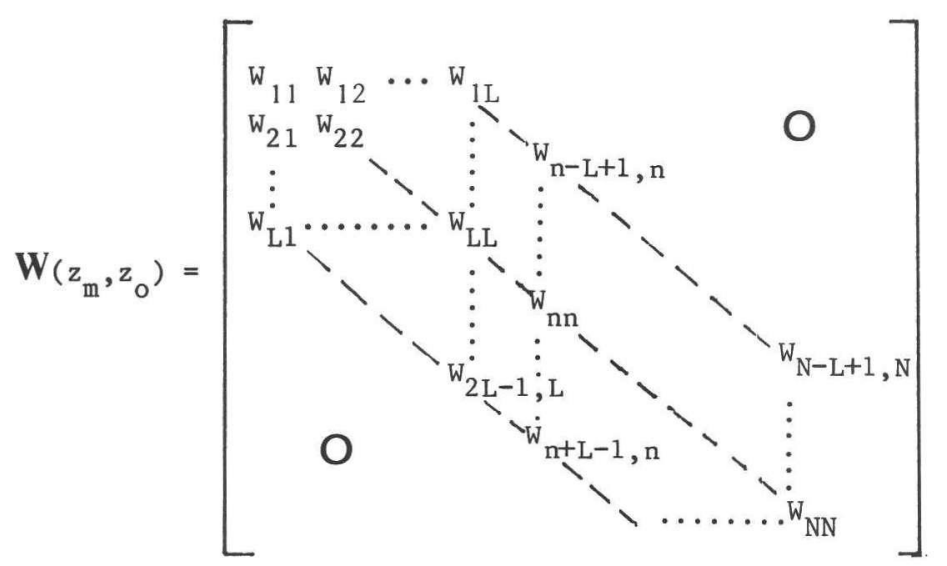

If there are no lateral velocity variations in the medium, all column vectors are shifted versions of each other, so that $W\left(z_{m}, z_{0}\right)$ is a $s y m e t r i c$ $T \circ$ e p 1 i t $z$ matrix: 


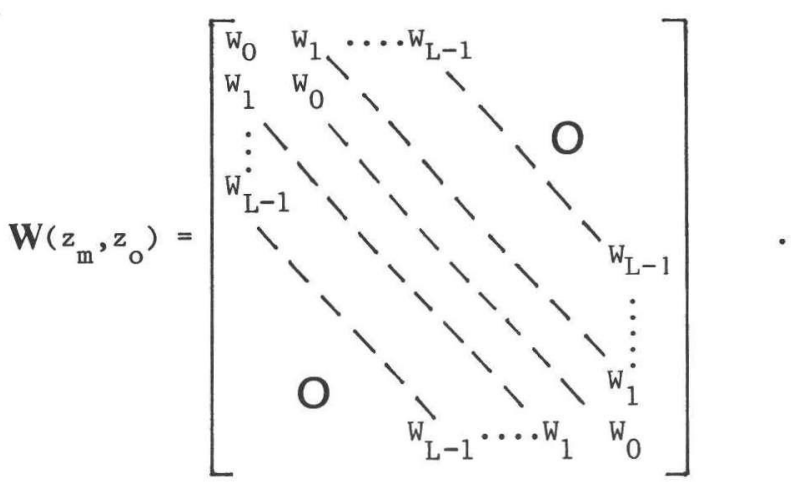

$(\mathrm{II}-7)$

In this case, the matrix representation of wave propagation can be replaced by a simpler notation since all columns now contain identical elements, representing a spatial wavelet $W$ which is invariant in source (centre) position $x_{q}$ :

$$
S\left(x_{p}, z_{m}, w_{i}\right)=\sum_{q} W\left(x_{p}-x_{q}, \Delta z_{m}, w_{i}\right) S\left(x_{q}, z_{o}, w_{i}\right) .
$$

This expression denotes a $s$ a $t i$ a 1 c $\circ \mathrm{n} v \circ 1$ u $t i \circ n$ in $x$, to be written as:

$$
S\left(x, z_{m}, w_{i}\right)=W\left(x, \Delta z_{m}, w_{i}\right) * S\left(x, z_{o}, \omega_{i}\right),
$$

see also Fig. II-5.

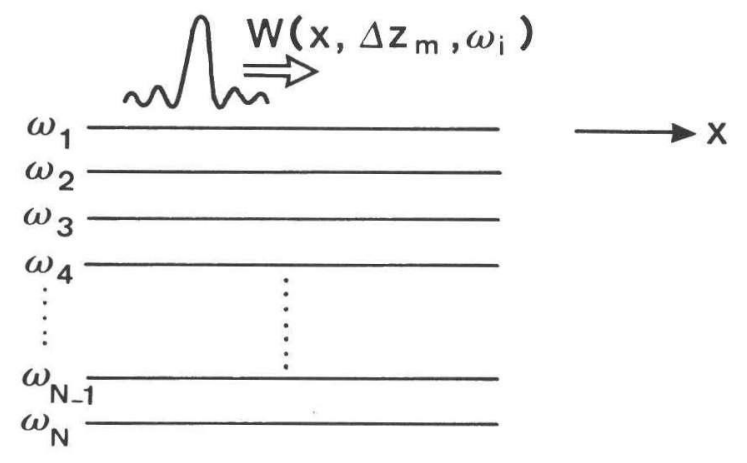

Figure II-5: Propagation from level $\mathrm{z}_{0}$ to level $\mathrm{z}_{\mathrm{m}}$ interpreted as a spatial convolution.

In this case of lateral homogeneity, the homogeneous operator, Eq. (II-5a), can be Fourier transformed (finite DFT) to the wavenumber-frequency domain, resulting in: 


$$
\begin{aligned}
& \widetilde{w}\left(k_{x}, \Delta z_{m}, \omega_{i}\right)=\exp \left[-j\left(k^{2}-k_{x}^{2}\right)^{\frac{1}{2}} \Delta z_{m}\right] \text { for } k_{x}^{2} \leqq k^{2}, \\
& \widetilde{w}\left(k_{x}, \Delta z_{m}, \omega_{i}\right)=\exp \left[-\left(k_{x}^{2}-k^{2}\right) \Delta z_{m}\right] \text { for } k_{x}^{2}>k^{2} .
\end{aligned}
$$

The spatial convolution given in Eq. (II-8b) is transformed to a m u $1 \mathrm{t} i$ p 1 i c a $t$ o $n$ in this domain:

$$
\widetilde{S}\left(k_{x}, z_{m}, \omega_{i}\right)=\widetilde{W}\left(k_{x}, \Delta_{m}, \omega_{i}\right) \widetilde{S}\left(k_{x}, z_{o}, \omega_{i}\right) .
$$

The spatial limitations of the rows and columns composing matrix $\mathbf{W}\left(\mathrm{z}_{\mathrm{m}}, \mathrm{z}_{\mathrm{o}}\right)$, together with the practical limitation of temporal bandwidth $\left(\omega_{i} \leqq \omega_{\max }\right)$ can be expressed in limitations of the spatial bandwidth of $\widetilde{w}\left(k_{x}, \Delta z_{m}, \omega_{i}\right)$ :

$$
\left|\mathrm{k}_{\mathrm{x}}\right| \leqq \mathrm{k}_{\mathrm{x}, \max }
$$

Hence, operator $W\left(x, \Delta z_{m}, w_{i}\right)$ is often called a b a n d - 1 i m i t e d spatial wavelet.

It should be mentioned here that, as Berkhout (1982, par. 6.2) derives, the wave propagation from one layer to another has a r e c u r s i v e character, which means in matrix notation:

$\mathbf{W}\left(z_{m}, z_{o}\right)=W\left(z_{m}, z_{m-1}\right) \mathbf{T}\left(z_{m-1}\right) W\left(z_{m-1}, z_{m-2}\right) \ldots W_{\left(z_{2}, z_{1}\right)} \mathbf{T}\left(z_{1}\right) \mathbf{W}\left(z_{1}, z_{o}\right) .($ II -12$)$

In this expression, matrix $\mathbf{T}\left(z_{1}\right)$ specifies the transmission properties of depth level $z_{1}$, etc. In this way, variations in the medium velocity in $\mathrm{ve} \mathrm{r}-$ t $i$ c a 1 direction can always be coped with.

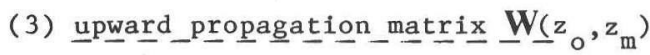

Matrix $W\left(z_{0}, z_{m}\right)$, describing the wave propagation from depth leve1 $z_{m}$ to sur-

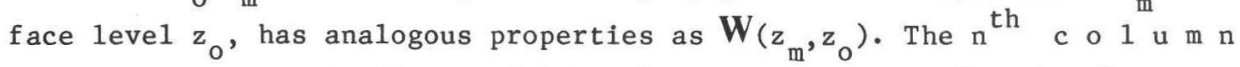
vector now represents the spatial impulse response at surface level $z_{0}$ to a source positioned in $\left(n \Delta x, z_{m}\right)$ - see Fig. II-6a - whereas the $n^{\text {th }} r \circ w$ vector gives the response in $\left(n \triangle x, z_{0}\right)$ to an array of dipole sources at depth leve1 $z_{m}$, Fig. II-6b. 


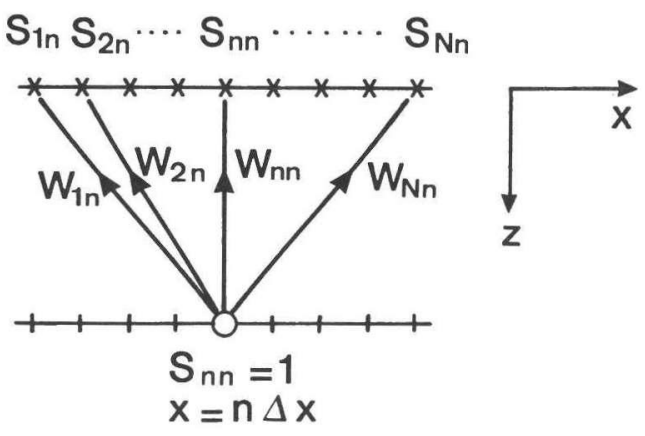

a

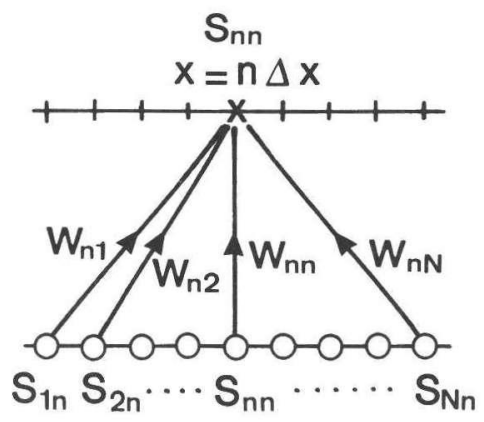

b

Figure II-6: Illustration to the interpretation of column vectors (a) and row vectors (b) of propagation matrix $\mathbf{W}\left(\mathrm{z}_{\mathrm{o}}, \mathrm{z}_{\mathrm{m}}\right)$.

It is simply seen by comparing Figs. II-3 and II-6 that, in the absence of vertical variations, $\mathbf{W}\left(\mathrm{z}_{\mathrm{o}}, \mathrm{z}_{\mathrm{m}}\right)$ can be derived from $\mathbf{W}\left(\mathrm{z}_{\mathrm{m}}, \mathrm{z}_{\mathrm{o}}\right)$ by interchanging rows and columns:

$$
\mathbf{W}\left(z_{0}, z_{m}\right)=W^{T}\left(z_{m}, z_{o}\right)
$$

If there are no lateral variations as well, $\mathbf{W}$ is symmetric and hence:

$$
W\left(z_{0}, z_{m}\right)=W\left(z_{m}, z_{o}\right)
$$

(4) reflectivity matrix $\underline{\mathbf{R}}\left(\mathrm{z}_{\mathrm{m}}\right)$

Matrix $\mathbf{R}\left(\mathrm{z}_{\mathrm{m}}\right)$ denotes the pressure reflectivity of depth level $\mathrm{z}_{\mathrm{m}}$, specifying which part of the incident pressure field - given by $\mathbf{S}\left(z_{m}\right)$ - is radiated back to the surface:

$$
\mathbf{P}\left(z_{m}\right)=\mathbf{R}\left(z_{m}\right) \mathbf{S}\left(z_{m}\right)
$$

Note that in general the reflectivity is dependent on frequency and angle of incidence.

Considering the $n^{\text {th }}$ column vector of $\mathbf{S}\left(\mathrm{z}_{\mathrm{m}}\right)$, denoting the incident pressure field around $\left(n \Delta x, z_{m}\right)$, we write: 


$$
\overrightarrow{\mathrm{P}}_{\mathrm{n}}\left(\mathrm{z}_{\mathrm{m}}\right)=\mathbf{R}\left(\mathrm{z}_{\mathrm{m}}\right) \overrightarrow{\mathrm{S}}_{\mathrm{n}}\left(\mathrm{z}_{\mathrm{m}}\right),
$$

or:

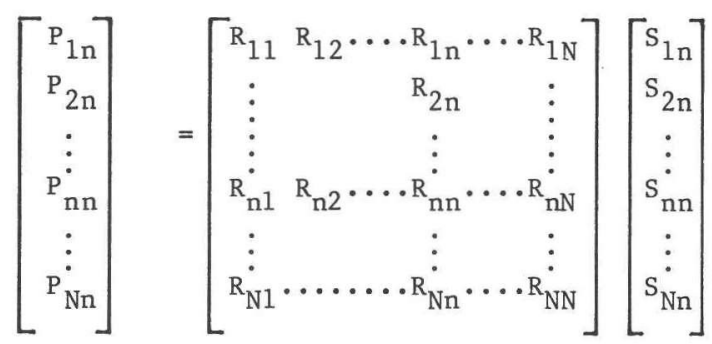

It is seen that the $\mathrm{n}^{\text {th }}$ c $\circ 1 \mathrm{um} \mathrm{n}$ of $\mathbf{R}\left(\mathrm{z}_{\mathrm{m}}\right)$ defines the contribution to $\overrightarrow{\mathrm{P}}_{n}\left(\mathrm{z}_{\mathrm{m}}\right)$ from the incident pressure at point $\left(\mathrm{n} \Delta \mathrm{x}, \mathrm{z}_{\mathrm{m}}\right)$, i.e. element $\mathrm{S}_{\mathrm{nn}}$, see Fig. II-7a. The $n^{\text {th }} r \circ w$ of $\mathbf{R}\left(z_{m}\right)$ specifies how the total incident field around $\left(\mathrm{n} \Delta \mathrm{x}, \mathrm{z}_{\mathrm{m}}\right)$ contributes to the reflected pressure $\mathrm{i} n$ that point, see Fig. II $-7 b$.

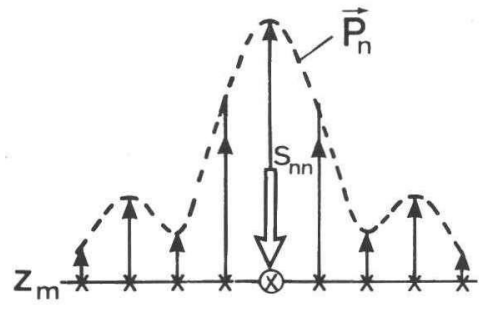

a

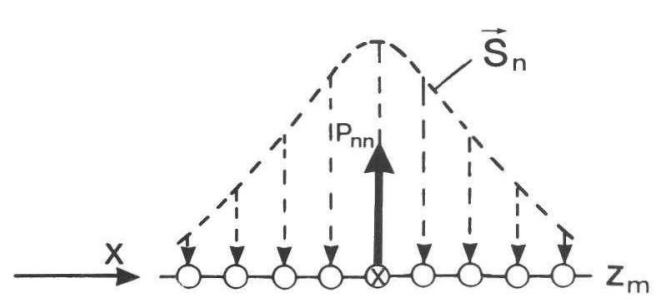

b

Figure II-7: Illustration to the interpretation of columns (a) and rows (b) of reflectivity matrix $\mathbf{R}\left(\mathrm{z}_{\mathrm{m}}\right)$.

Hence, the $n^{\text {th }}$ row of $\mathbf{R}\left(\mathrm{z}_{\mathrm{m}}\right)$ defines the angle-dependent reflection coefficient at $\left(n \Delta x, z_{m}\right)$. In the special case that the reflection coefficient is not angle-dependent, the subsurface is 'locally reacting': $P_{n n}$ is determined by $\mathrm{s}_{\mathrm{nn}}$ on1y and, hence, $\mathbf{R}\left(\mathrm{z}_{\mathrm{m}}\right)$ is a diagonal matrix.

(5) detector matrix $\underline{\text { D }}\left(\mathrm{z}_{0}\right)$

Matrix $\mathbf{D}\left(z_{0}\right)$ determines the sensitivity and directivity properties of the detector arrays at the surface. The $n^{\text {th }} r$ o w of $\mathbf{D}\left(z_{0}\right)$ specifies those properties for an array around point $\left(n \triangle x, z_{0}\right)$. Hence, for an arrangement of single detectors $\mathbf{D}\left(z_{0}\right)$ is a diagonal matrix. 


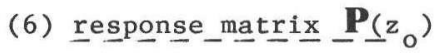

Matrix $\mathbf{P}\left(z_{0}\right)$ represents the multi-trace primary pressure data at surface level $z_{0}$. Element $P_{p q}$ specifies the pressure contribution at detector (centre) position $\left(\mathrm{p} \Delta \mathrm{x}, \mathrm{z}_{\mathrm{o}}\right)$ due to a source at - or a source array around point $\left(\mathrm{q} \Delta \mathrm{x}, \mathrm{z}_{\mathrm{o}}\right)$. Hence, as was illustrated in Fig. I-4:

- each column of matrix $\mathbf{P}\left(z_{0}\right)$ represents a s o u r c e gather, i.e. the response of all detectors to one source,

- each row represents a d e $t$ e $c t o r$ gather, i.e. the response of one detector to a11 sources,

- the main antidiagonal represents the $c \circ m m \circ n$ m $i d p i n t$ gather around the aperture centre, $i . e$. the responses of detectors in $\left(p \Delta x, z_{0}\right)$ to sources in $\left(-p \Delta x, z_{0}\right)$, where $p=-K,-(K-1), \ldots,-1,0,1, \ldots, k-1, K$; the other antidiagonals represent common midpoint gathers around other aperture points,

- the main diagonal represents the $z$ e $r$ o - of f s e t data, i.e. the responses of detectors placed at the same positions as the sources,

- the subdiagonals represent $c \circ m m \circ n$ of $\mathrm{s}$ e $t$ sections, for which the offset between sources and detectors is constant.

Note that, if only zero-offset data are recorded, $\mathbf{P}\left(z_{0}\right)$ is a diagonal matrix.

\section{Final remark:}

In this section, we considered a two-dimensional model. The same treatment holds for the three-dimensional situation, where each matrix element represents a vector. The homogeneous operator, describing the wave propagation between points $\left(\mathrm{x}_{\mathrm{A}}, \mathrm{y}_{\mathrm{A}}, \mathrm{z}_{\mathrm{o}}\right)$ and $\left(\mathrm{x}_{\mathrm{B}}, \mathrm{y}_{\mathrm{B}}, \mathrm{z}_{\mathrm{m}}\right)$ then reads:

$$
W\left(x, y, z, w_{i}\right)=\frac{\Delta z_{m}}{2 \pi} \frac{1+j k r}{r^{3}} \exp (-j k r),
$$

with

$$
\mathrm{r}^{2}=\left(\mathrm{x}_{\mathrm{A}}-\mathrm{x}_{\mathrm{B}}\right)^{2}+\left(\mathrm{y}_{\mathrm{A}}-\mathrm{y}_{\mathrm{B}}\right)^{2}+\left(\Delta_{\mathrm{m}}\right)^{2}
$$

The double Fourier transform to the wavenumber-frequency domain reads:

$$
\begin{aligned}
& \widetilde{W}\left(k_{x}, k_{y}, z, \omega_{i}\right)=\exp \left[-j\left(k^{2}-k_{x}^{2}-k_{y}^{2}\right)^{\frac{1}{2}} \Delta_{m}\right] \text { for } k_{x}^{2}+k_{y}^{2} \leq k^{2}, \quad \text { (II-18a) } \\
& \widetilde{W}\left(k_{x}, k_{y}, z, \omega_{i}\right)=\exp \left[-\left(k_{x}^{2}+k_{y}^{2}-k^{2}\right)^{\frac{1}{2}} \Delta_{m}\right] \quad \text { for } k_{x}^{2}+k_{y}^{2}>k^{2}, \quad \text { (II-18b) }
\end{aligned}
$$




\section{II.3 MODELING AND INVERSION IN ZERO-OFFSET TECHNIQUES}

\section{II.3.1. Adaptation of the physical primary model to the zero-offset configuration}

If data acquisition is performed in $z$ e $r$ o o f $f \mathrm{~s} t$, for each physical experiment source and detector are placed at the same point. Hence, assuming point sources and detectors, the matrices $\mathbf{S}\left(z_{0}\right)$ and $\mathbf{D}\left(z_{0}\right)$ are diagonal matrices. In zero-offset techniques, the reflectivity of any boundary may, with good approximation ${ }^{1}$, be described as 'locally reacting', since only one angle of incidence is of importance, viz. the angle normal to the boundary in the point considered. Hence, the reflection coefficient is quasi angle-independent, which corresponds with local reaction. Hence, for zero-offset acquisition also $\mathbf{R}\left(\mathrm{z}_{\mathrm{m}}\right)$ can be described as a diagonal matrix.

Taking into account the diagonal properties of $\mathbf{S}\left(\mathrm{z}_{0}\right), \mathbf{D}\left(\mathrm{z}_{\mathrm{o}}\right)$ and $\mathbf{R}\left(\mathrm{z}_{\mathrm{m}}\right)$, we now write, for the response of one depth level $z_{m}$, the general matrix equation

$$
\mathbf{P}\left(\mathrm{z}_{\mathrm{o}}\right)=\mathbf{D}\left(\mathrm{z}_{\mathrm{o}}\right) \mathbf{W}\left(\mathrm{z}_{\mathrm{o}}, \mathrm{z}_{\mathrm{m}}\right) \mathbf{R}\left(\mathrm{z}_{\mathrm{m}}\right) \mathbf{W}\left(\mathrm{z}_{\mathrm{m}}, \mathrm{z}_{\mathrm{o}}\right) \mathbf{S}\left(\mathrm{z}_{\mathrm{o}}\right)
$$

as:

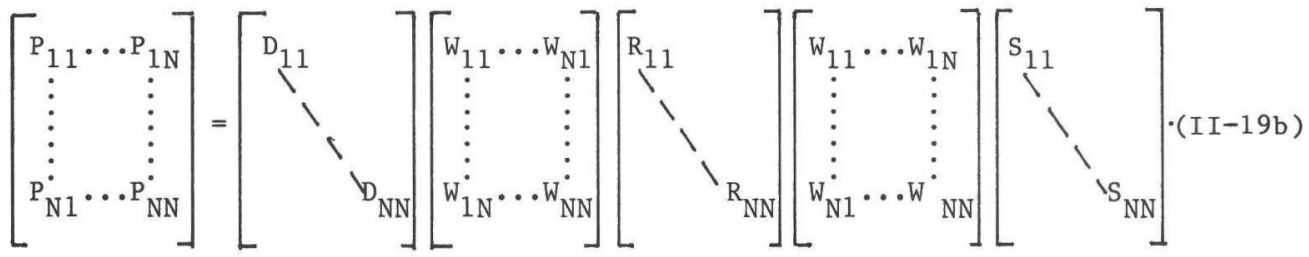

The diagonal source and detector matrices can be included in the propagation matrices as weighting functions, hence:

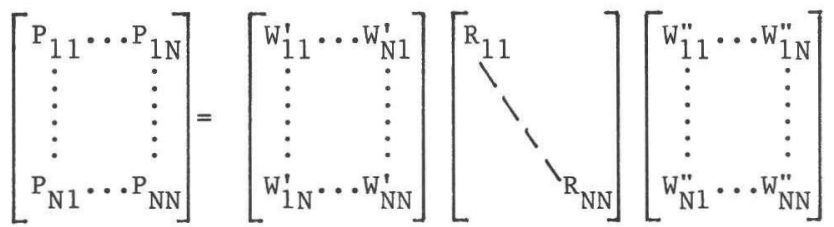

1) Here, the $c u r v a t u r e$ of the incident wavefront is neglected, which introduces an error $-\mathbf{R}\left(z_{m}\right)$ has some non-zero subdiagonals - which in most practical situations has no significant influence. 
In zero-offset acquisition techniques, only the $m$ a $i \mathrm{n} d \mathrm{i} g$ o $\mathrm{n}$ a 1 of response matrix $\mathbf{P}\left(z_{0}\right)$ is considered. Due to the diagonal property of matrix $\mathbf{R}\left(z_{m}\right)$, the diagonal elements of $\mathbf{P}\left(z_{0}\right)$ can be written as:

$$
\mathrm{P}_{\mathrm{mm}}=\sum_{i} \mathrm{~W}_{\mathrm{im}}^{\prime} \mathrm{W}_{\mathrm{im}}^{\prime \prime} \mathrm{R}_{\mathrm{ii}}
$$

Using the subscript $z O$ to indicate the zero-offset situation we write Eq. (II-20) in matrix notation as follows:

$$
\overrightarrow{\mathrm{P}}_{Z O}\left(z_{0}\right)=\mathbf{W}_{Z O}\left(z_{0}, z_{\mathrm{m}}\right) \overrightarrow{\mathrm{R}}_{\mathrm{ZO}}\left(\mathrm{z}_{\mathrm{m}}\right)
$$

where

$$
\begin{aligned}
& \overrightarrow{\mathrm{P}}_{\mathrm{ZO}}\left(\mathrm{z}_{0}\right)=\left(\mathrm{P}_{11}, \mathrm{P}_{22}, \ldots, \mathrm{P}_{\mathrm{NN}}\right)^{\mathrm{T}}, \\
& \overrightarrow{\mathrm{R}}_{\mathrm{ZO}}\left(\mathrm{z}_{\mathrm{m}}\right)=\left(\mathrm{R}_{11}, \mathrm{R}_{22}, \ldots, \mathrm{R}_{\mathrm{NN}}\right)^{\mathrm{T}},
\end{aligned}
$$

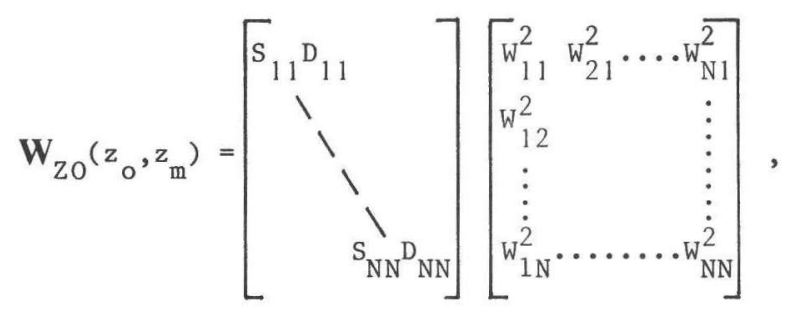

$W_{p q}$ being given by the homogeneous extrapolation operator WII, Eq. (II-5a), and superscript ()$^{\mathrm{T}}$ denoting transposition.

If there are no lateral velocity variations in the medium, matrix equation (II-21a) can be replaced by a s p a t a 1 c o n vol u t i o n - see also Fig. II-5 - so that the zero-offset primary pressure response of a two-dimensional configuration of $m$ depth layers can be written as:

$$
\mathrm{P}_{\mathrm{ZO}}\left(\mathrm{x}, \mathrm{z}_{\mathrm{o}}, \omega_{\mathrm{i}}\right)=\mathrm{S}\left(\mathrm{w}_{\mathrm{i}}\right) \sum_{\mathrm{m}} \mathrm{W}_{\mathrm{ZO}}\left(\mathrm{x}, \Delta_{\mathrm{m}}, \mathrm{w}_{\mathrm{i}}\right) * \mathrm{R}_{\mathrm{ZO}}\left(\mathrm{x}, \mathrm{z}_{\mathrm{m}}, \mathrm{w}_{\mathrm{i}}\right)
$$

where $S\left(\omega_{i}\right)$ denotes the spectral strength of the source-detector combination and $\mathrm{W}_{\mathrm{ZO}}\left(\mathrm{x}, \Delta_{\mathrm{z}_{\mathrm{m}}}, \omega_{i}\right)$ is the squared WII-operator. Throughout our discussion of zero-offset techniques - which play a dominant role in the following chapters we shall use this notation in the form of a convolution, even if there are lateral velocity variations. Then, the operator has to be interpreted as a space-variant convolution. 
For a two-dimensional homogeneous medium, the zero-offset extrapolation operator $\mathrm{W}_{\mathrm{ZO}}\left(\mathrm{x}, \Delta_{\mathrm{m}}, \mathrm{w}_{\mathrm{i}}\right)$ reads:

$$
\begin{aligned}
W_{Z O}\left(x, \Delta z_{m}, \omega_{i}\right) & =w^{2}\left(x, \Delta z_{m}, \omega_{i}\right) \\
& =\left(\Delta z_{m}\right)^{2} \frac{j k}{2 \pi} \frac{\exp (-2 j k r)}{r^{3}}
\end{aligned}
$$

which is not a solution of the linear wave equation. Berkhout (1982, sect. 6.8) shows that in practical situations, $W_{Z 0}\left(x, \Delta z_{m}, \omega_{i}\right)$ may be approximated with sufficient precision by the non-squared homogeneous operator WII after substitution of the d o u b 1 e frequency value or the $h$ a $1 \mathrm{f}$ velocity value:

$$
\begin{aligned}
W_{Z O}\left(x, \Delta z_{m}, \omega_{i}\right) & \approx W\left(x, \Delta z_{m}, 2 \omega_{i}\right) \\
& =\Delta z_{m} \sqrt{\frac{j k}{\pi}} \frac{\exp (-2 j k r)}{r^{3 / 2}} .
\end{aligned}
$$

Hence, the spatial Fourier transform of $\mathrm{W}_{\mathrm{ZO}}\left(\mathrm{x}, \Delta \mathrm{z}_{\mathrm{m}}, \omega_{i}\right)$ is approximated by:

$$
\begin{array}{lll}
\widetilde{\mathrm{W}}_{\mathrm{ZO}}\left(\mathrm{k}_{\mathrm{x}}, \Delta \mathrm{z}_{\mathrm{m}}, \omega_{\mathrm{i}}\right) \approx \exp \left[-\mathrm{j}\left(4 \mathrm{k}^{2}-\mathrm{k}_{\mathrm{x}}^{2}\right)^{\frac{1}{2}} \Delta \mathrm{z}_{\mathrm{m}}\right] & \text { for } \mathrm{k}_{\mathrm{x}}^{2} \leq 4 \mathrm{k}^{2}, & \text { (II-25a) } \\
\widetilde{\mathrm{W}}_{\mathrm{ZO}}\left(\mathrm{k}_{\mathrm{x}}, \Delta \mathrm{z}_{\mathrm{m}}, \omega_{\mathrm{i}}\right) \approx \exp \left[-\left(\mathrm{k}_{\mathrm{x}}^{2}-4 \mathrm{k}^{2}\right)^{\frac{1}{2}} \Delta \mathrm{z}_{\mathrm{m}}\right] & \text { for } \mathrm{k}_{\mathrm{x}}^{2}>4 \mathrm{k}^{2} . & \text { (II-25b) }
\end{array}
$$

It should be realized that we now introduced a $\mathrm{m}$ a $\mathrm{t}$ h e $\mathrm{m}$ a $\mathrm{t}$ i $\mathrm{c}$ a 1 model of the physical situation, covering the depth planes with 'mathematical dipoles', the strength of which is given by reflectivity distribution $\mathrm{R}_{\mathrm{ZO}}\left(\mathrm{x}, \mathrm{z}_{\mathrm{m}}, \mathrm{w}_{\mathrm{i}}\right)$.

Two final remarks should be made here:

(1) Berkhout (1982, section 6.7 ) shows that replacement of propagation matrices $\mathbf{W}\left(z_{0}, z_{m}\right)$ and $\mathbf{W}\left(z_{m}, z_{0}\right)$ by one two-way propagation matrix is also possible for $\mathrm{c} \circ \mathrm{mm}$ o $\mathrm{n}$ of $\mathrm{f}$ e $t$ data. Hence, considerations on processing of zero-offset data can easily be extended to common offset data by replacing matrix $\mathbf{W}_{\mathrm{ZO}}$ (or spatial wavelet $\mathrm{W}_{\mathrm{ZO}}$ ) by a matrix $\mathbf{W}_{\mathrm{CO}}$ (or spatial wavelet $\mathrm{W}_{\mathrm{CO}}$ ) representing two-way propagation of common offset data.

(2) Fig. II-8 shows that modeling the zero-offset response of a single diffractor and modeling a $c \circ m m o n m i d p o i n t$ gather on a horizontal $r$ e $f 1$ e $c$ t o $r$ involve identical travel times yielding similar 
h y p e r b 01 i c responses. This means that spatial wavelet $\mathrm{W}_{\mathrm{ZO}}$ describing wave propagation in the first situation is identical to spatial wavelet $\mathrm{W}_{\mathrm{CMP}}$ applying to the second situation. Hence, considerations on processing of zero-offset diffractor responses can easily be extended to hyperbolic CMP gathers of reflection data.

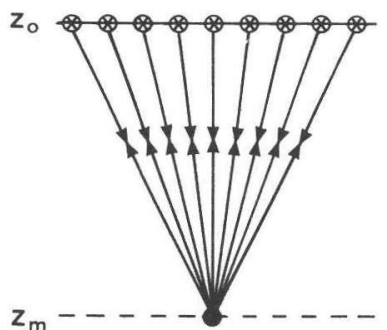

a

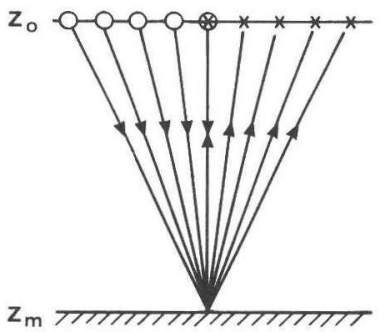

$\mathrm{C}$

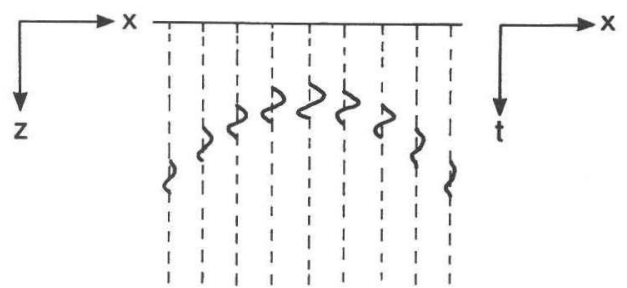

b

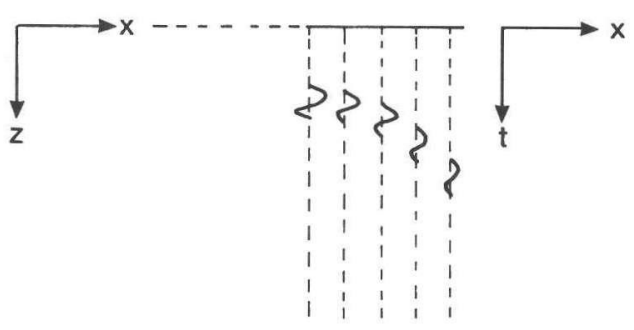

d

Figure II-8: Analogy between zero-offset diffraction data and common midpoint reflection data.

a: ray geometry of zero-offset modeling of a single diffractor below a homogeneous layer

b: zero-offset response of a single diffractor

c: ray geometry of CMP modeling of a horizontal reflector below a homogeneous layer

d: CMP gather on a horizontal reflector

\section{II.3.2. Inversion scheme for zero-offset data}

In subsection II.2.2 it was made clear that, in general, an inversion process involves a sequence of matrix inversions (i.e. solution of a series of linear equations) in order to solve the reflectivity matrices $\mathbf{R}\left(\mathrm{z}_{\mathrm{m}}\right)$ from matrix 
equation (II-1). Since, however, for $z$ e $r \circ-\circ f f s$ e $t$ primary data Eq. (II-1) can be written in the form of a single spatial convolution, Eq. (II-22), inversion of zero-offset data only requires a single d e c o n v $01 \mathrm{u}-$ $t i \circ n$ procedure in order to eliminate the effects of propagation operator $\mathrm{W}_{\mathrm{ZO}}\left(\mathrm{x}, \Delta \mathrm{z}_{\mathrm{m}}, \omega_{i}\right)$. Hence, omitting for simplicity the spectral factor $\mathrm{S}\left(\omega_{i}\right)$, for each depth level $\mathrm{z}_{\mathrm{m}}$ an estimate of reflectivity distribution $\mathrm{R}_{\mathrm{ZO}}\left(\mathrm{x}, \mathrm{z}_{\mathrm{m}}, \omega_{\mathrm{i}}\right)$ is represented by:

$$
\begin{aligned}
\left\langle\mathrm{R}_{\mathrm{ZO}}\left(\mathrm{x}, \mathrm{z}_{\mathrm{m}}, \omega_{i}\right)\right\rangle & =\mathrm{F}\left(\mathrm{x}, \Delta_{\mathrm{m}}, \omega_{i}\right) * \mathrm{P}_{\mathrm{ZO}}\left(\mathrm{x}, \mathrm{z}_{\mathrm{o}}, \omega_{i}\right) \\
& =\mathrm{F}\left(\mathrm{x}, \Delta_{\mathrm{m}}, \omega_{i}\right) * \mathrm{~W}_{\mathrm{ZO}}\left(\mathrm{x}, \Delta_{\mathrm{m}}, \omega_{i}\right) * \mathrm{R}_{\mathrm{ZO}}\left(\mathrm{x}, \mathrm{z}_{\mathrm{m}}, \omega_{i}\right),
\end{aligned}
$$

where $F\left(x, \Delta z_{m}, \omega_{i}\right)$ is to be interpreted as a spatial deconvolution operator. It is clear that a fully correct estimate of the reflectivity distribution is obtained if, and only if:

$$
\mathrm{F}\left(\mathrm{x}, \Delta_{\mathrm{m}}, \mathrm{w}_{\mathrm{i}}\right) * \mathrm{~W}_{\mathrm{Z} 0}\left(\mathrm{x}, \Delta_{\mathrm{m}}, \mathrm{w}_{\mathrm{i}}\right)=\delta(\mathrm{x}),
$$

which in the wavenumber-frequency domain corresponds with:

$$
\widetilde{F}\left(k_{x}, \Delta z_{m}, \omega_{i}\right) \widetilde{W}_{Z O}\left(k_{x}, \Delta z_{m}, \omega_{i}\right)=1 \text {, for all } k_{x} \cdot
$$

Substitution of Eq. (II-25) into Eq. (II-28) yields:

$$
\begin{aligned}
& \widetilde{F}\left(k_{x}, \Delta z_{m}, \omega_{i}\right)=\exp \left[j\left(4 k^{2}-k_{x}^{2}\right)^{\frac{1}{2}} \Delta z_{m}\right] \text { for } k_{x}^{2} \leqq 4 k^{2}, \\
& \widetilde{F}\left(k_{x}, \Delta z_{m}, \omega_{i}\right)=\exp \left[+\left(k_{x}^{2}-4 k^{2}\right)^{\frac{1}{2}} \Delta z_{m}\right] \text { for } k_{x}^{2}>4 k^{2} .
\end{aligned}
$$

Eq. (II-29b) shows that this operator has an unstable character: evanescent components of the recorded field are 'blown up'. Besides, the propagation operator $\mathrm{W}_{Z O}\left(\mathrm{x}, \Delta \mathrm{z}_{\mathrm{m}}, \omega_{i}\right)$ is usually spatially band-limited due to aperture and frequency limitations, and of ten has a smaller spatial bandwidth than the always-present spatially coherent noise. For $k_{x}$-values where the signal-tonoise ratio is low, this noise is also 'blown up' by a broadband deconvolution operator.

Hence, conditions (II-27,28) can never be reached so that a perfect estimate of reflectivity distribution $\mathrm{R}_{\mathrm{zO}}\left(\mathrm{x}, \mathrm{z}_{\mathrm{m}}, \omega_{\mathrm{i}}\right)$ can never be obtained. Berkhout 
(1982, section 7.2) proposes three alternatives to achieve an o $\mathrm{p} t \mathrm{i}$ a 1 estimate:

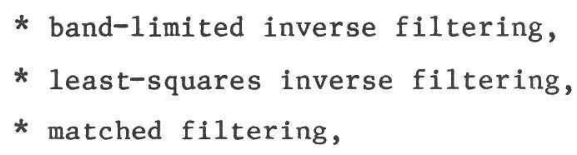

expressing preference for the last technique because of its simplicity and practical applicability. Properties of inversion operators based on the acoustic wave equation are also discussed by De Vries and Berkhout (1981).

In $m$ a $t \mathrm{ch}$ e $\mathrm{f} i \mathrm{t}$ e $\mathrm{r} i \mathrm{n} \mathrm{g}$, the deconvolution operator is chosen, in the wavenumber-frequency domain, as:

$$
\widetilde{\mathrm{F}}\left(\mathrm{k}_{\mathrm{x}}, \Delta \mathrm{z}_{\mathrm{m}}, \omega_{i}\right)=\widetilde{\mathrm{W}}_{\mathrm{ZO}}^{*}\left(\mathrm{k}_{\mathrm{x}}, \Delta \mathrm{z}_{\mathrm{m}}, \omega_{i}\right),
$$

where ( $)^{*}$ denotes complex conjugation. Hence, for zero-offset data without spatial bandwidth limitation, we find according to Eq. (II-25):

$$
\begin{aligned}
& \widetilde{F}\left(k_{x}, \Delta z_{m}, \omega_{i}\right)=\exp \left[j\left(4 k^{2}-k_{x}^{2}\right)^{\frac{1}{2}} \Delta z_{m}\right] \quad \text { for } k_{x}^{2} \leqq 4 k^{2}, \quad \text { (II-31a) } \\
& \widetilde{F}\left(k_{x}, \Delta z_{m}, \omega_{i}\right)=\exp \left[-\left(k_{x}^{2}-4 k^{2}\right)^{\frac{1}{2}} \Delta z_{m}\right] \quad \text { for } k_{x}^{2}>4 k^{2} \text {. (II-31b) }
\end{aligned}
$$

Fourier transformation yields the following expression in the space-frequency domain:

$$
F\left(x, \Delta z_{m}, \omega_{i}\right)=\Delta z_{m} \sqrt{\frac{-j k}{\pi}} \frac{\exp (2 j k r)}{r^{3 / 2}} .
$$

It is seen that for proper matched filtering the correct wavenumber value $k$ and, hence, the correct velocity distribution in layer $\Delta z_{m}$ should be inserted into the deconvolution operator. Then, neglecting absorption effects, matched filtering represents a pure $s$ a $t i$ a 11 y $z$ e $r o-p h$ a $s$ i $g$ procedure within the spatial bandwidth of interest ${ }^{1}$ ). Hence, for the matched

1) In practice, data acquisition is performed in an aperture of finite length which means that operator $\mathrm{W}_{\mathrm{ZO}}\left(\mathrm{x}, \Delta \mathrm{z}_{\mathrm{m}}, \mathrm{w}_{\mathrm{i}}\right)$ is multiplied with a box-window and, hence, $\widetilde{W}_{z 0}\left(k_{x}, \Delta z_{m}, \omega_{i}\right)$ is convolved with a sinc-function. This convolution leads to a ripple in the spatial amplitude spectrum known as the Gibbs-phenomenon, which can be suppressed by spatial windowing. Although, hence, $\left|\widetilde{W}_{Z O}\right|$ is not exactly white within the spatial bandwidth considered, the white filter $\widetilde{F}$ is used for inversion. 
filtered result we write:

$$
\begin{aligned}
& \tilde{\mathrm{U}}_{\mathrm{z} 0}\left(\mathrm{k}_{\mathrm{x}}, \mathrm{z}_{\mathrm{m}}, \omega_{i}\right) \\
& \left.\tilde{\mathrm{F}}_{\mathrm{x}}, \mathrm{k}_{\mathrm{z}}, \mathrm{w}_{\mathrm{i}}\right) \tilde{\mathrm{W}}_{\mathrm{Z} 0}\left(\mathrm{k}_{\mathrm{x}}, \Delta_{\mathrm{z}_{\mathrm{m}}}, \omega_{i}\right)=1,\left|\mathrm{k}_{\mathrm{x}}\right| \leqq \mathrm{k}_{\mathrm{x}, \max }<2 \mathrm{k},
\end{aligned}
$$

and hence:

$$
\begin{aligned}
& \mathrm{U}_{\mathrm{ZO}}\left(\mathrm{x}, \mathrm{z}_{\mathrm{m}}, \mathrm{w}_{\mathrm{i}}\right) \\
& \mathrm{F}\left(\mathrm{x}, \Delta_{\mathrm{z}_{\mathrm{m}}}, \omega_{i}\right) * \mathrm{~W}_{\mathrm{Z} 0}\left(\mathrm{x}, \Delta_{\mathrm{z}_{\mathrm{m}}}, \mathrm{w}_{\mathrm{i}}\right)=\text { const. } \frac{\sin \left(\mathrm{k}_{\mathrm{x}, \max } \mathrm{x}\right)}{\mathrm{k}_{\mathrm{x}, \max } \mathrm{x}},
\end{aligned}
$$

which is a real zero-phase function. If, however, erroneous velocity values are chosen, the deconvolved result is no longer zero-phase, which means that the dispersion of the resulting spatial wavelet increases and, hence, the lateral resolution of the estimated reflectivity distribution decreases. These effects will be discussed in more detail in chapter III.

Finally, we present two migration schemes for zero-offset data based on matched filtering, viz. a recursive and a non-recursive scheme. The $r$ e c u r s i v e scheme is formulated by Berkhout (1982, section 7.9) and applies if velocity variations must be taken into account, The medium is divided into depth layers such that, within the aperture, these layers can be considered as homogeneous. Since the wave field is migrated layer by layer, this recursive technique is also called a 's t r i p p i n g ' technique. For each depth level, the reflectivity distribution is estimated in the space-frequency domain:

$$
\begin{aligned}
& \left\langle\mathrm{R}_{\mathrm{ZO}}\left(\mathrm{x}, \mathrm{z}_{1}, \omega_{\mathrm{i}}\right)\right\rangle=\mathrm{W}^{*}\left(\mathrm{x}, \Delta \mathrm{z}_{1}, 2 \omega_{i}\right) * \mathrm{P}_{\mathrm{ZO}}\left(\mathrm{x}, \mathrm{z}_{\mathrm{o}}, \omega_{\mathrm{i}}\right) \\
& \left.<\mathrm{R}_{\mathrm{ZO}}\left(\mathrm{x}, \mathrm{z}_{2}, \omega_{\mathbf{i}}\right)=\mathrm{w}^{*}\left(\mathrm{x}, \Delta \mathrm{z}_{2}, 2 \omega_{i}\right) *<\mathrm{R}_{\mathrm{ZO}}\left(\mathrm{x}, \mathrm{z}_{1}, \omega_{\mathrm{i}}\right)\right\rangle \\
& \begin{array}{c}
\vdots \\
\vdots \\
\left\langle\mathrm{R}_{\mathrm{ZO}}\left(\mathrm{x}, \mathrm{z}_{\mathrm{m}}, \omega_{1}\right)\right\rangle \mathrm{W}^{*}\left(\mathrm{x}, \Delta \mathrm{z}_{\mathrm{m}}, 2 \omega_{i}\right) *\left\langle\mathrm{R}_{\mathrm{ZO}}\left(\mathrm{x}, \mathrm{z}_{\mathrm{m}-1}, \omega_{i}\right)\right\rangle
\end{array}
\end{aligned}
$$

with

$$
\Delta \mathrm{z}_{\mathrm{m}}=\left|\mathrm{z}_{\mathrm{m}}{ }^{-\mathrm{z}_{\mathrm{m}-1}}\right|
$$


Imaging is performed by Fourier transformation of each estimated reflectivity distribution to the space-time domain, taking the value for $t=0$ :

$$
\left\langle\mathrm{r}_{\mathrm{ZO}}\left(\mathrm{x}, \mathrm{z}_{\mathrm{m}}, \mathrm{t}=0\right)\right\rangle=\frac{1}{\pi} \operatorname{Re} \int_{\omega_{\min }}^{\omega}\left\langle\mathrm{R}_{\mathrm{ZO}}\left(\mathrm{x}, \mathrm{z}_{\mathrm{m}}, \omega_{i}\right)\right\rangle \mathrm{d} \omega .
$$

$N \circ n-r$ e c u r $\mathrm{s} v$ e schemes can be applied if, within the entire aperture, a constant velocity may be used. Then, migration can be performed in the wavenumber-frequency domain, using a simple and fast algorithm. In one depth step, the medium is imaged down to the deepest level of interest, so that such techniques are also known as ' $\mathrm{m}$ a $\mathrm{p}$ p i $\mathrm{ng}$ ' techniques. The scheme given here is based on a paper by Stolt (1978) and formulated by Berkhout (1982, section 8.5). Basically, the scheme calculates the wave field at $t=0$ for all depth levels using the matched filtering operator in the wave-frequency domain given in Eq. (II-29), as follows:

$$
\begin{aligned}
p(x, z, t=0) & =\frac{1}{2 \pi} \int_{\omega} P(x, z, \omega) d \omega \\
& =\left(\frac{1}{2 \pi}\right)^{2} \int_{\omega} d \omega \int_{k_{x}}\left[\widetilde{P}\left(k_{x}, 0, \omega\right) \exp \left\{j\left(4 k^{2}-k_{x}^{2}\right)^{\frac{3}{2}} z\right\}\right] \exp \left(-j k_{x} x\right) d k_{\dot{x}}
\end{aligned}
$$

Introducing $\mathrm{k}_{\mathrm{z}}$ according to:

$$
\begin{aligned}
k_{z} & =\left(4 k^{2}-k_{x}^{2}\right)^{\frac{1}{2}} \\
& =\left(4 \frac{\omega^{2}}{c^{2}}-k_{x}^{2}\right)^{\frac{1}{2}},
\end{aligned}
$$

Eq. (II-36) is rewritten as:

$p(x, z, t=0)=\left(\frac{1}{2 \pi}\right)^{2} \int_{k_{z}} \exp \left(j k_{z} z\right) d k_{z} \int_{k_{x}} \frac{k_{z}}{\left(k_{z}^{2}+k_{x}^{2}\right)^{\frac{1}{2}}} \tilde{P}^{\prime}\left(k_{z}, 0, k_{z}\right) \exp \left(-j k_{x} x\right) d k_{x}$,

with $\quad \widetilde{P}^{\prime}\left(k_{x}, 0, k_{z}\right)=\frac{c}{2} \widetilde{P}\left(k_{x}, 0, \frac{c}{2} \sqrt{k_{z}^{2}+k_{x}^{2}}\right)$.

The mapping procedure as formulated by Eq. (II-38a) is performed in four steps: (1) A double Fourier transform is applied to the recorded data:

$$
\mathrm{p}(\mathrm{x}, 0, t) \stackrel{\mathrm{FT}}{\Rightarrow} \mathrm{P}(\mathrm{x}, 0, \omega) \stackrel{\mathrm{FT}^{-1}}{\Rightarrow} \widetilde{\mathrm{P}}\left(\mathrm{k}_{\mathrm{x}}, 0, \omega\right) \text {. }
$$




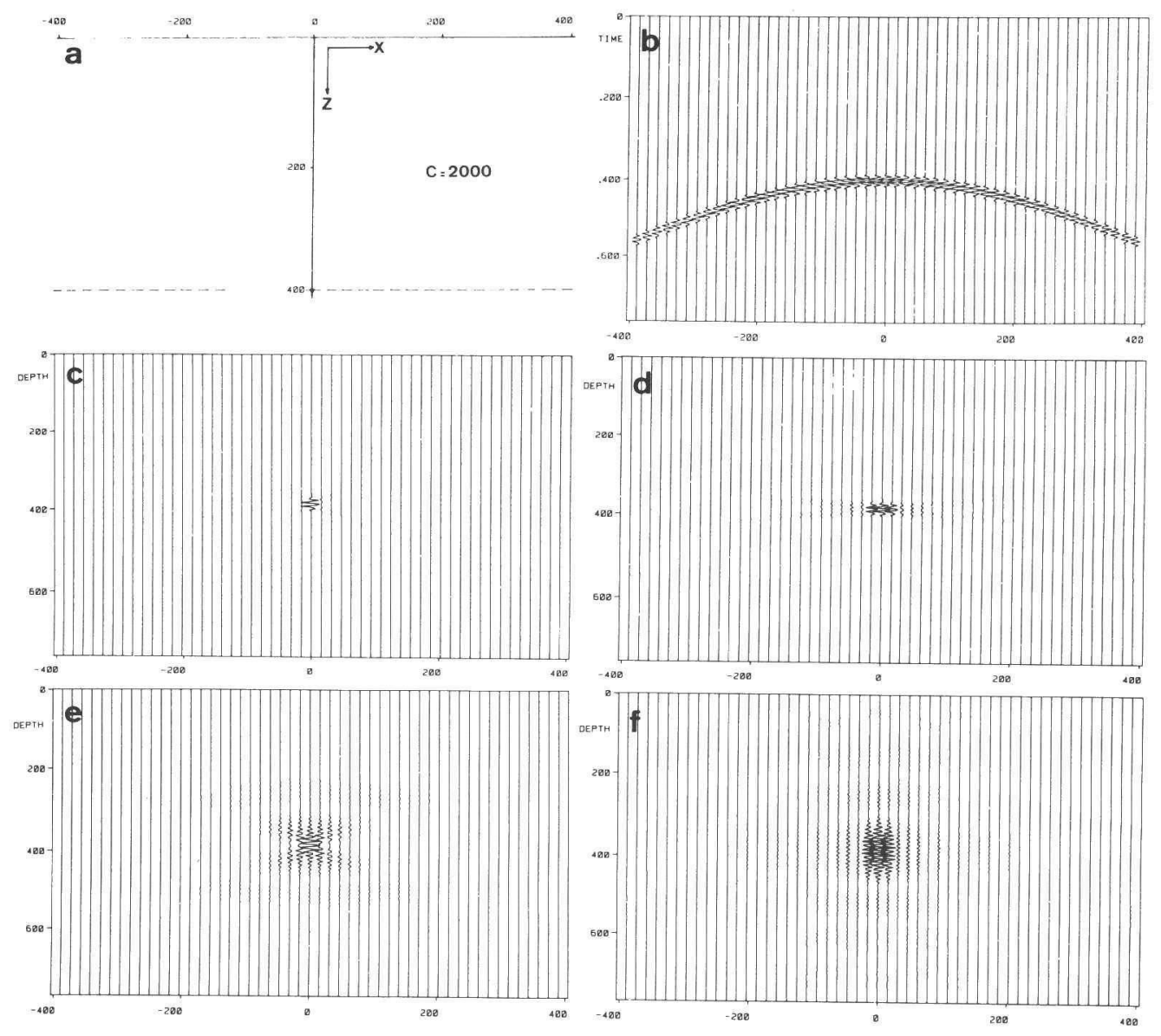

Figure II-9: Response and images of a dipole diffractor.

a: geometry

b: zero-offset response

c: optimal image obtained by matched filtering

d: image for limited spatial bandwidth

e: image for limited temporal bandwidth

f: image for limited spatial and temporal bandwidth 
(2) $\widetilde{P}\left(k_{x}, 0, w\right)$ is transformed to the $\left(k_{x}, k_{z}\right)$ domain according to $\mathrm{Eq} \cdot(\mathrm{II}-38 \mathrm{~b})$ :

$$
\widetilde{\mathrm{P}}\left(\mathrm{k}_{\mathrm{x}}, 0, \omega\right) \Rightarrow \widetilde{\mathrm{P}}^{\prime}\left(\mathrm{k}_{\mathrm{x}}, 0, \mathrm{k}_{\mathrm{z}}\right) \text {. }
$$

(3) $\widetilde{\mathrm{P}}^{\prime}\left(\mathrm{k}_{\mathrm{x}}, 0, \mathrm{k}_{z}\right)$ is multiplied with the weighting factor $\mathrm{k}_{z} /\left(\mathrm{k}_{z}^{2}+\mathrm{k}_{\mathrm{x}}^{2}\right)^{\frac{1}{2}}$.

(4) To the result of step (3) a double Fourier transform is applied:

$$
\frac{k_{z}}{\left(k_{z}^{2}+k_{x}^{2}\right)^{\frac{1}{2}}} \widetilde{P}^{\prime}\left(k_{x}, 0, k_{z}\right) \stackrel{F T}{\Rightarrow} P^{\prime \prime}\left(x, 0, k_{z}\right) \stackrel{F^{-1}}{\Rightarrow} p(x, z, t=0) .
$$

\section{II.4 EXAMPLES}

In order to conclude this chapter in an illustrative way, examples will be shown of forward as well as inverse processing of simulated data.

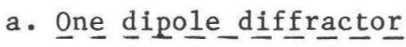

In Fig. II-9a, the geometry is given of a configuration consisting of a single dipole diffractor positioned centrally below a line aperture. Fig. II-9b shows the modeled zero-offset pressure response, using a broadband source pulse $\left(\omega_{\min }<\omega<\omega_{\max }\right) \cdot$ Fig. II-9c shows the imaged result in the space-time domain after matched filtering as described in subsection II.3.2, using full spatial and temporal bandwidth. Figs. II-9d,e,f show the images after bandwidth 1imitation: in Fig. II-9d the spatial bandwidth is limited to $\left|k_{x}\right| \leq k \sin (\pi / 12)$, in Fig. II-9e the temporal bandwidth has been reduced with a factor 10 around the original central frequency, in Fig. II-9f both limitations are applied simultaneously. It is seen that $s p$ a $t i$ a 1 bandwidth limitation leads to 1 a $t e^{-}$ $r$ a 1 broadening of the image, whereas $t$ e m p o r a 1 bandwidth limitation gives rise to dispersion in $\mathrm{v}$ e $\mathrm{r} t \mathrm{i}$ a 1 direction ${ }^{1)}$.

It should be noted that the data displayed in Fig. II-9 can be physically interpreted in two different ways (see also subsection II.3.1):

(1) as zero-offset data obtained from a point diffractor representing a local element of a density contrast,

1) However, since temporal and spatial frequency are related through wavenumber $k$, there is mutual influence between temporal and spatial bandwidth limitation, as is seen in the figures. 

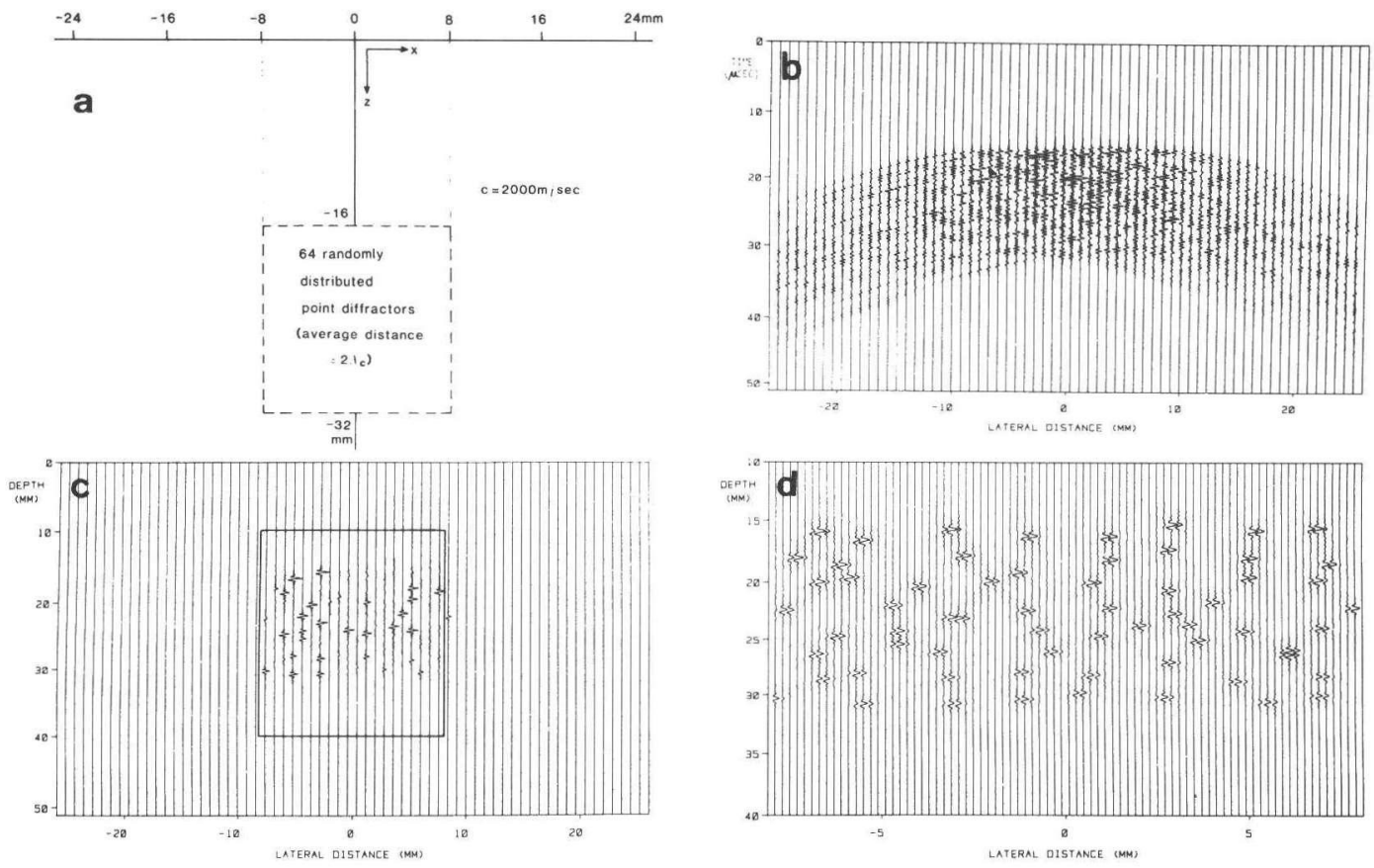

Figure II-10: Response and image of a diffractor distribution.

a: geometry

b: zero-offset response

c: mapped image

d: mapped image, enlarged

In $b$ and $c$ only one of each five traces has been plotted, whereas in $d$ a11 traces are presented. 

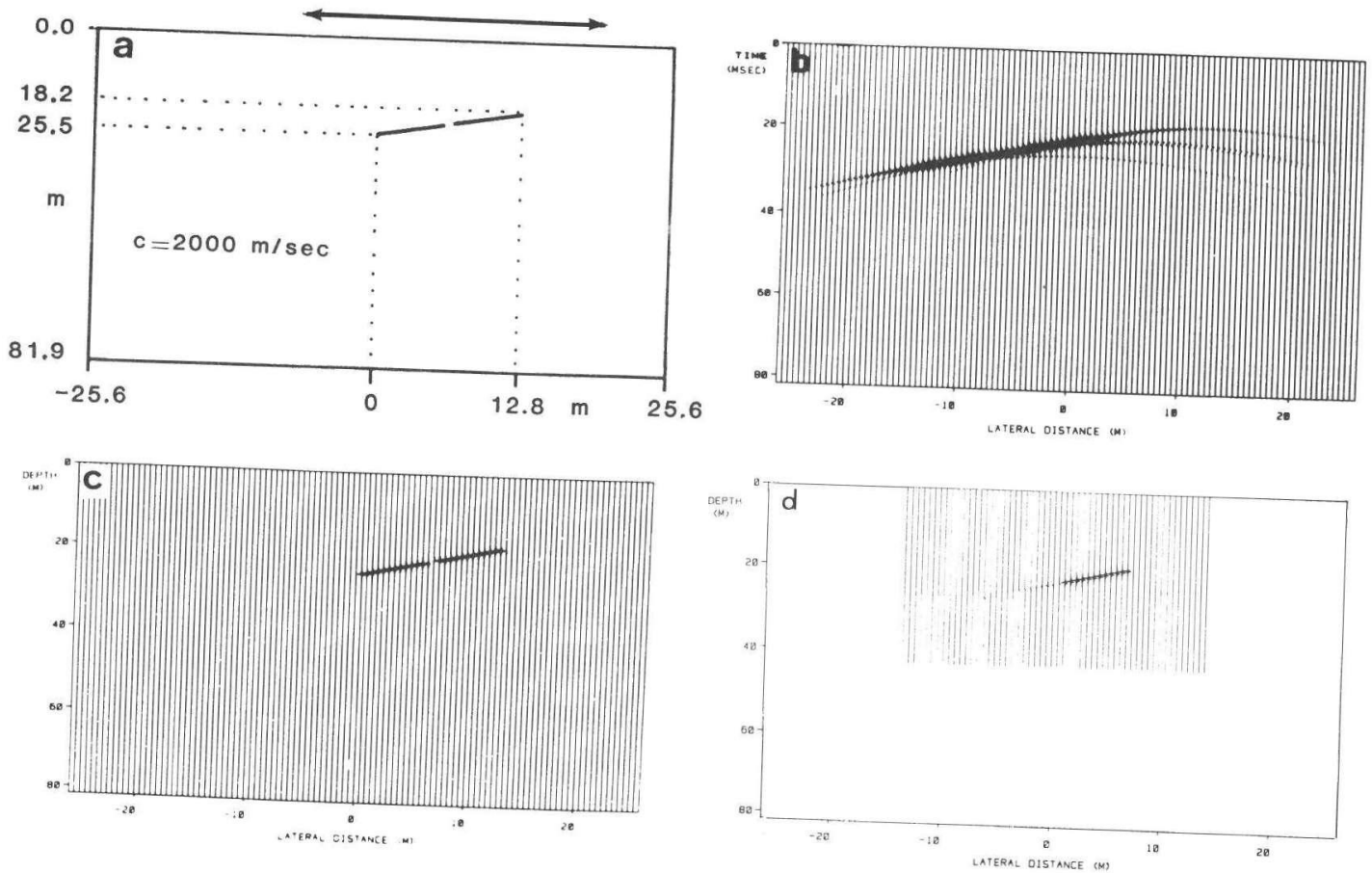

Figure II-11: Response and image of a dipping finite reflector with a central 'reflectivity drop'.

a: geometry

b: zero-offset response

c: mapped image

d: mapped image after aperture limitation 
(2) as a common midpoint gather obtained from a locally reacting plane reflector representing a density contrast between two layers.

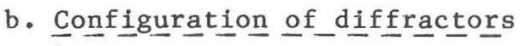

Fig. II-10a shows a configuration of 64 dipole diffractors, randomly distributed over a square depth field below an aperture line. Fig. II-10b shows the modeled zero-offset response and Figs. II-10c,d the imaged result after nonrecursive mapping in the wavenumber-frequency domain, using full spatial and temporal bandwidth.

\section{c. Dipping reflecte-tor}

Fig. II-1la shows the geometry of a finite reflector with a tilt angle $\left(\alpha=30^{\circ}\right)$ in respect to the aperture line and a small opening ('reflectivity drop') in the centre. Fig. II-11b gives the zero-offset response at the aperture line. Fig. II-1lc displays the imaged result obtained with non-recursive mapping in the wavenumber-frequency domain. Comparison of Figs. II-11a and $b$ shows that the zero-offset response of a dipping reflector 'shifts away' in lateral direction from the reflector position. Hence, if the response is recorded within an aperture covering the reflector area only - as indicated above Fig. II-11a - only a limited part of the reflected wave field is acquired. Inversion of this windowed response yields an image which does not resemble the reflector model, as is shown in Fig. II-11d. Hence, for imaging of dipping reflectors wide apertures must be chosen. 


\section{CHAPTER III:}

\section{INFLUENCE OF VELOCITY ERRORS ON ACOUSTIC INVERSION}

\section{III.1. INTRODUCTION}

In chapter II it has been shown that proper data inversion, e.g. by application of spatial matched filtering, leads to an image having a spatial zero-phase spectrum. However, this is only true if the correct velocity distribution of the medium is inserted into the inversion operator. In this chapter, the effects of velocity errors on the inverted result in various domains will be discussed, especially for zero-offset data.

III.2. INFLUENCE OF VELOCITY ERRORS ON THE SPATIAL WAVELET RESULTING AFTER MATCHED FILTERING

In subsection II.3.2 we have shown that, for absorption-free media, matched filtering is a spatial zero-phasing operation, i.e. the resulting spatial wavelet has a spatial zero-phase spectrum. For zero-offset data from a depth leve1 $\mathrm{z}$ we derived, see Eq. (II-33a):

$$
\left.\begin{array}{l}
\widetilde{U}_{Z 0}\left(k_{x}, z, \omega_{i}\right)= \\
\widetilde{F}_{x}\left(k_{x}, \omega_{i}\right) \widetilde{W}_{Z O}\left(k_{x}, z, \omega_{i}\right)= \\
\widetilde{W}_{Z 0}^{*}\left(k_{x}, z, \omega_{i}\right) \widetilde{W}_{Z O}\left(k_{x}, z, \omega_{i}\right)= \\
\exp \left(j k_{z} z\right) \exp \left(-j k_{z} z\right)=1,
\end{array}\right\}\left|k_{x}\right| \leqq k_{x, \max }<2 k \quad \text { (III-1a) }
$$

with

$$
k_{z}=\left(4 k^{2}-k_{x}^{2}\right)^{\frac{1}{2}}
$$



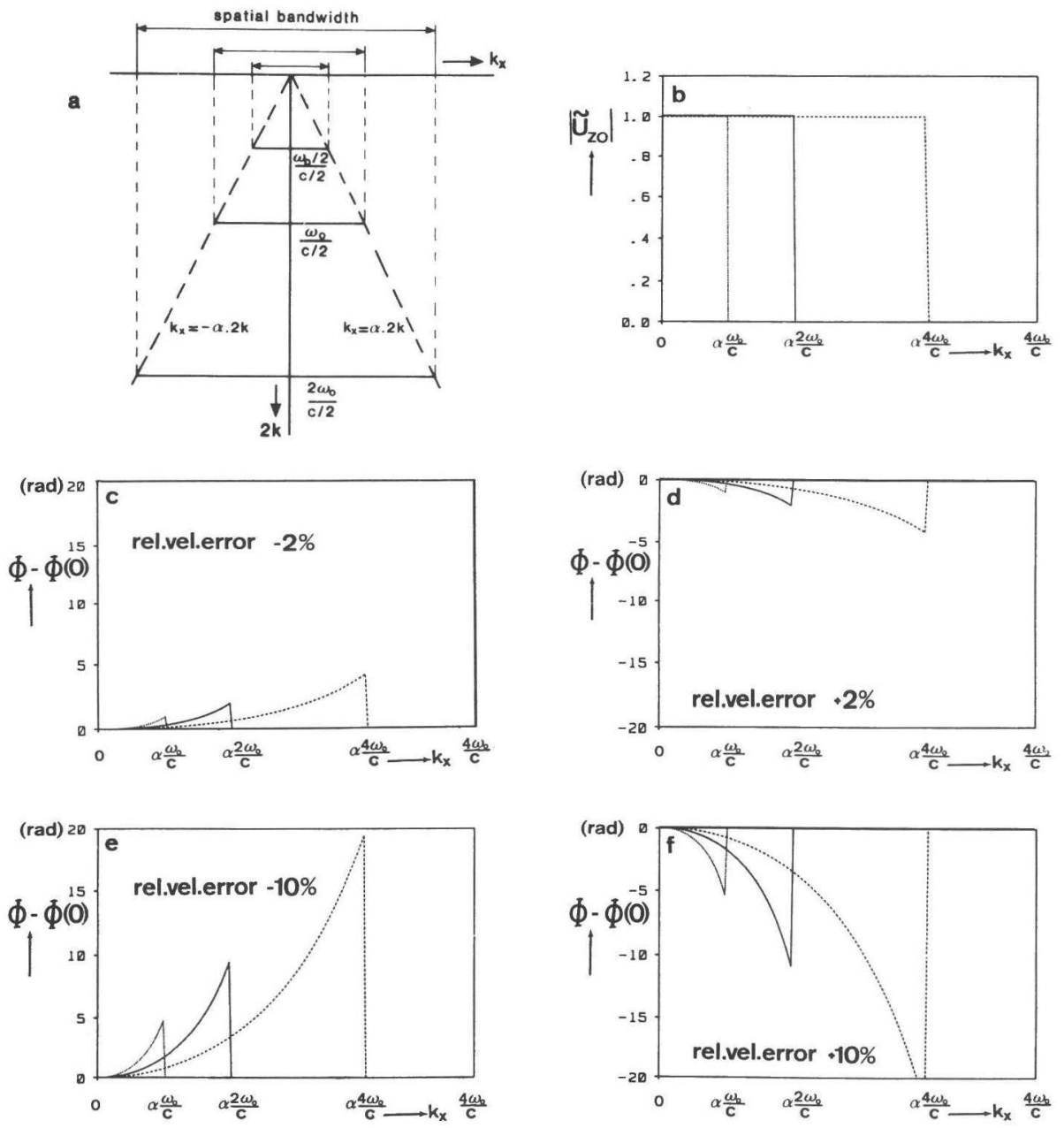

Figure III-1: Spatial phase spectra $\Phi\left(\mathrm{k}_{\mathrm{x}}\right)$ of $\tilde{\mathrm{U}}_{\mathrm{ZO}}\left(\mathrm{k}_{\mathrm{x}}, \mathrm{z}=20 \lambda_{0}, \omega_{i}\right)$ for various velocity errors and $\omega_{i}=\frac{1}{2} \omega_{0}, \omega_{0}, 2 \omega_{0}, \lambda_{0}$ being the wavelength for $\omega_{0}$. Bandwidth parameter $\alpha=\frac{1}{2} \sqrt{2}$.

a: spatial bandwidth as a function of frequency ( $k_{x}^{-k}$ diagram)

b: spatial amplitude spectra

c: $\Phi\left(k_{x}\right)-\Phi(0)$ for relative velocity error $-2 \%$

d: id., $+2 \%$

e: id., $-10 \%$

f: id., $+10 \%$

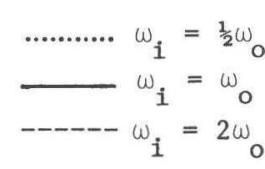


If, however, instead of the true velocity value $c$ an e $r \mathrm{r}$ o $\mathrm{n}$ e o u $\mathrm{s}$ value $c^{\prime}$ is inserted into operator $\widetilde{F}\left(k_{x}, z, \omega_{i}\right)$, the spatial amplitude spectrum of $\widetilde{U}_{Z O}\left(k_{x}, z, \omega_{i}\right)$ remains unaffected but the spatial $p$ h a $s$ e spectrum is no longer zero:

$$
\widetilde{U}_{Z 0}\left(k_{x}, z, \omega_{i}\right)=\exp \left[j\left(k_{z}^{\prime} z-k_{z} z\right)\right],\left|k_{x}\right| \leqq k_{x, \max }<2 k
$$

with

$$
\begin{aligned}
& k_{z}^{\prime}=\left(4 k^{\prime 2}-k_{x}^{2}\right)^{\frac{1}{2}}, \\
& k^{\prime}=\omega_{i} / c^{\prime} .
\end{aligned}
$$

It is seen that the error in the spatial phase angle increases with depth level $z$. The influence of other parameters $\left(\omega_{i}, c^{\prime}-c\right)$ becomes clearer if we consider the approximation of Eq. (III-2) for $\left|k_{x}\right|<<k$, which corresponds with sma11 aperture angles. Then:

$$
\begin{aligned}
& \mathrm{k}_{\mathrm{z}} \approx 2 \mathrm{k}-\frac{\mathrm{k}_{\mathrm{x}}^{2}}{4 \mathrm{k}}, \\
& \mathrm{k}_{\mathrm{z}}^{\prime} \approx 2 \mathrm{k}^{\prime}-\frac{\mathrm{k}_{\mathrm{x}}^{2}}{4 \mathrm{k}^{\prime}},
\end{aligned}
$$

and hence

$$
\widetilde{U}_{z 0}\left(k_{x}, z, \omega_{i}\right) \approx \exp \left[j\left\{2 \omega_{i}\left(\frac{1}{c^{\prime}}-\frac{1}{c}\right)-\frac{k_{x}^{2}}{4 \omega_{i}}\left(c^{\prime}-c\right)\right\} z\right] .
$$

It is now seen that:

(1) the spatial phase angle has a non-zero value for $k_{x}=0$, the sign of which depends on the sign of the velocity error. The absolute value depends on the velocity value as well as the velocity error and increases with frequency.

(2) the spatial phase angle increases or decreases - depending on the velocity error sign - with $\left|k_{x}\right|$ in a parabolic sense, the 'steepness' depending on absolute velocity error and frequency.

The above considerations are illustrated in Fig. III-1 and Table III/1. Function $\widetilde{U}_{z 0}\left(k_{x}, z, \omega_{i}\right)$ as given by Eq. (III-2a) is considered for three frequencies $\left(\omega_{i}=\frac{1}{2} \omega_{0}, \omega_{0}\right.$ and $\left.2 \omega_{0}\right)$ and a depth level $z=20 \lambda_{0}, \lambda_{0}$ being the wavelength corresponding with $\omega_{\circ}$. For each frequency, $\widetilde{Z}_{\mathrm{ZO}}$ is band-1imited such that $\left|k_{x}\right| \leqq 2 \alpha k$ where $\alpha$ is chosen $\frac{1}{2} \sqrt{2}$. Fig. III-la shows how the spatial bandwidth increases with frequency. Fig. III-lb gives the spatial amplitude 


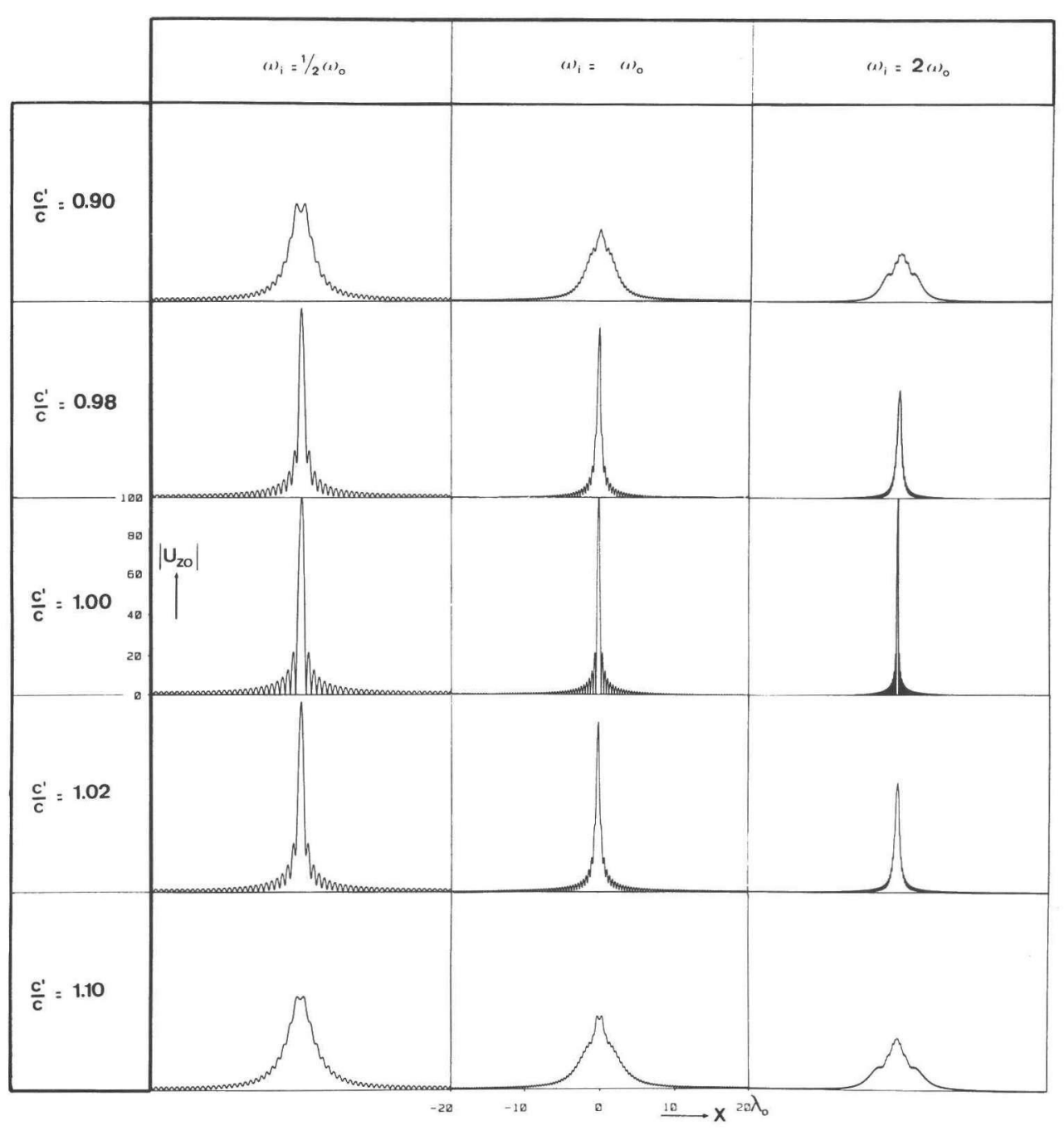

Figure III-2: Directivity patterns of spatial wavelet $U_{Z O}\left(x, z, \omega_{i}\right)$ for various velocity error and frequency values; $\lambda_{0}=2 \pi \mathrm{c} / \omega_{0}$. 
spectra for the three frequencies, being white within the bandwidth considered. Figs. III-lc,d,e,f show how spatial phase $\Phi\left(\mathrm{k}_{\mathrm{x}}\right)$ changes with $\mathrm{k}_{\mathrm{x}}$, in respect to the values $\Phi(0)$ for $k_{x}=0$, for velocity errors of $-2 \%,+2 \%,-10 \%$ and $+10 \%$ respectively. In each figure, the results for the three frequencies considered are displayed. The values of $\Phi(0)$ are given in Table III/1, for all frequency and velocity error combinations. As expected from Eq. (III-3c), for a given velocity error $\Phi(0)$ is proportional with frequency.

\section{Table III/1}

Spatial phase $\Phi(0)$ of $\widetilde{U}_{Z 0}$ for $k_{x}=0$, for velocity errors and frequencies considered in Fig. III-1.

\begin{tabular}{|c|c|c|c|}
\hline 7 & $\frac{1}{2} \omega_{0}$ & $\omega_{0}$ & $2 \omega_{0}$ \\
\hline .90 & $4.44 \pi$ & $8.88 \pi$ & $17.78 \pi$ \\
\hline .98 & $0.82 \pi$ & $1.63 \pi$ & $3.27 \pi$ \\
\hline 1.02 & $-0.78 \pi$ & $-1.57 \pi$ & $-3.14 \pi$ \\
\hline 1.10 & $-3.64 \pi$ & $-7.27 \pi$ & $-14.55 \pi$ \\
\hline
\end{tabular}

In Fig. III-2, the modulus patterns are given of the corresponding spatial wavelets $U_{Z 0}\left(x, z, w_{i}\right)$, i.e. the spatial Fourier transform of Eq. (III-2a). Note that these figures can be interpreted as $d i r$ e $t i v i t y$ patterns. In case of correct velocity, the modulus value for $x=0$ has been normalized to 100 for each frequency.

The following phenomena are evidently shown:

(1) The lateral d is p e r s i o $n$ of the directivity patterns increases with increasing velocity error.

(2) If the correct velocity value is used, the lateral dispersion of the directivity patterns decreases with increasing frequency, as expected. In case of velocity errors, however, this frequency-dependence vanishes.

(3) For a given frequency, the amplitude for $x=0$ decreases with increasing velocity error, and even may no longer form the absolute maximum of the pattern. This is in agreement with the well-known fact - see e.g. Berkhout (1974) - that a two-sided signal has maximum amplitude for $x=0$ if the phase spectrum is zero. The decrease of amplitude for $x=0$ due to velocity errors increases with frequency.

(4) As can be seen in Eq. (III-3c), $\widetilde{U}_{\mathrm{ZO}}$ is not a symmetric function of velocity error $c^{\prime}-c$. Hence, directivity patterns and phase spectra are not congruent for velocity errors with opposite sign. 
III.3. INFLUENCE OF VELOCITY ERRORS ON THE INVERTED RESULT IN THE SPACETIME DOMAIN

In the $s \mathrm{p}$ a $\mathrm{c}$ e - f $\mathrm{r}$ e $\mathrm{q} u$ e $\mathrm{n} \mathrm{y}$ domain, the spatial wavelet resulting after deconvolution of zero-offset propagation operator $\omega_{z 0}\left(x, z, k=\omega_{i} / c\right)$ with a matched filter $F\left(x, z, k^{\prime}=\omega_{i} / c^{\prime}\right)$ reads:

$$
\mathrm{U}_{\mathrm{ZO}}\left(\mathrm{x}, \mathrm{z}, \mathrm{w}_{\mathrm{i}}\right)=\mathrm{F}\left(\mathrm{x}, \mathrm{z}, \mathrm{k}^{\prime}\right) * \mathrm{~W}_{\mathrm{ZO}}(\mathrm{x}, \mathrm{z}, \mathrm{k})
$$

or, applying the coordinates indicated in Fig. III-3:

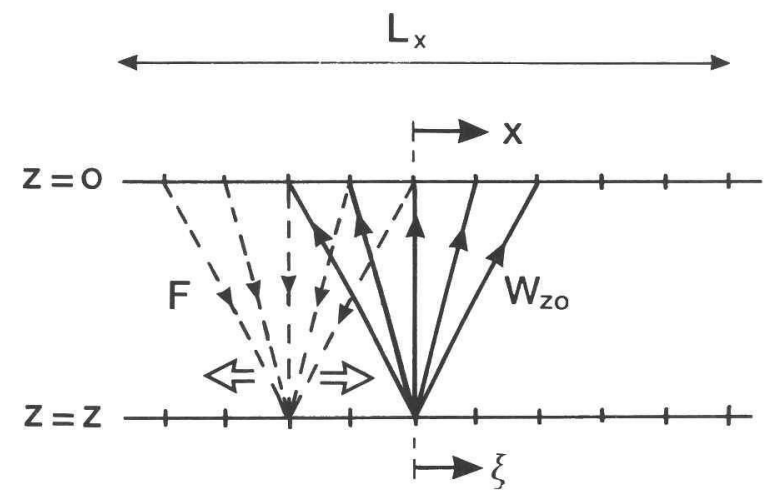

Figure III-3: Illustration to Eq. (III-4).

$$
\mathrm{U}_{\mathrm{ZO}}\left(\xi, z, \omega_{i}\right)=\int_{\mathrm{L}_{\mathrm{X}}(\mathrm{z}=0)} \mathrm{F}\left(\mathrm{x}-\xi, z, \mathrm{k}^{\prime}\right) \mathrm{W}_{\mathrm{ZO}}(\mathrm{x}, \mathrm{z}, \mathrm{k}) \mathrm{dx}
$$

or, substituting Eq. (II-30):

with

$$
\begin{aligned}
U_{Z O}\left(\xi, z, \omega_{i}\right) & =\int_{L_{X}(z=0)} W_{Z O}^{*}\left(x-\xi, z, k^{\prime}\right) W_{Z O}(x, z, k) d x \\
& =\int_{L_{X}} \sqrt{k^{\prime} k} \frac{z^{2}}{\pi} \frac{\exp \left(2 j k^{\prime} \rho\right)}{\rho^{3 / 2}} \frac{\exp (-2 j k r)}{r^{3 / 2}} d x
\end{aligned}
$$

$$
\begin{aligned}
& r^{2}=\left[x^{2}+z^{2}\right]^{\frac{1}{2}}, \\
& \rho^{2}=\left[(x-\xi)^{2}+z^{2}\right]^{\frac{1}{2}} .
\end{aligned}
$$


It is seen that, in the space-frequency domain, the dipole pressure field given by $W_{z O}(x, z, k)$ is submitted to a m p 1 i t u d e we i g h t i n g (apodization) through the factor $\rho^{-3 / 2}$ as well as $\mathrm{p}$ h a s e c $\circ \mathrm{r} r$ e c$t i \circ n$ through the factor $\exp \left(2 j k^{\prime} \rho\right)$. Choosing $\xi=0$, the resulting phase factor is $\exp \left[-2 j\left(k-k^{\prime}\right) r\right]$. In absence of velocity errors $k^{\prime}$ equals $k$, the contributions to the integral in $\mathrm{Eq}$. (III-4c) have equal (viz. zero) phase for a11 $x$ and hence $U_{Z O}\left(\xi, z, \omega_{i}\right)$ has maximum value for $\xi=0$.

Next, we Fourier transform Eq. (III-4c) to the $s p a c e-t i m e$ domain for $\xi=0$, using the shift theorem

$$
F(\omega) \exp (-j k r) \stackrel{F T}{\Longleftrightarrow} f\left(t-\frac{r}{c}\right) .
$$

Then:

$$
\mathrm{U}_{\mathrm{ZO}}(0, \mathrm{z}, \mathrm{t})=\frac{\mathrm{z}^{2}}{\pi \sqrt{\mathrm{c}^{\prime} \mathrm{c}}} \int_{L_{\mathrm{x}}(\mathrm{z}=0)} \frac{1}{\mathrm{r}^{3}} \mathrm{f}\left\{t-2\left(\frac{\mathrm{r}}{\mathrm{c}^{\prime}}-\frac{\mathrm{r}}{\mathrm{c}}\right)\right\} \mathrm{dx}
$$

where $f(t)$ is the inverse Fourier transform of the function

$$
\mathbf{F}(\omega)=\omega \cdot
$$

Eq. (III-6a) expresses that, apart from amplitude weighting, inversion can in the space-time domain be interpreted as a $t \mathrm{r}$ a v e 1 t $i \mathrm{~m}$ e $\mathrm{c} \circ \mathrm{m} \mathrm{p}$ e $s$ a $t i \circ n$ procedure: for each aperture coordinate $x$, a fraction $2 \mathrm{r} / \mathrm{c}^{\prime}$ is subtracted from signal arrival time $2 \mathrm{r} / \mathrm{c}$. In the following, this is discussed in more detail.

The travel times from a secondary dipole source at $(\xi=0, z)$ are given by

$$
\begin{aligned}
\tau(x) & =\frac{\left(x^{2}+z^{2}\right)^{\frac{1}{2}}}{c / 2} \\
& =\frac{2 r}{c}
\end{aligned}
$$

and, hence, form the $h y p$ e b o 1 a drawn as a solid curve in Fig. III-4, with asymptotes

$$
\tau= \pm \frac{2 x}{c}
$$




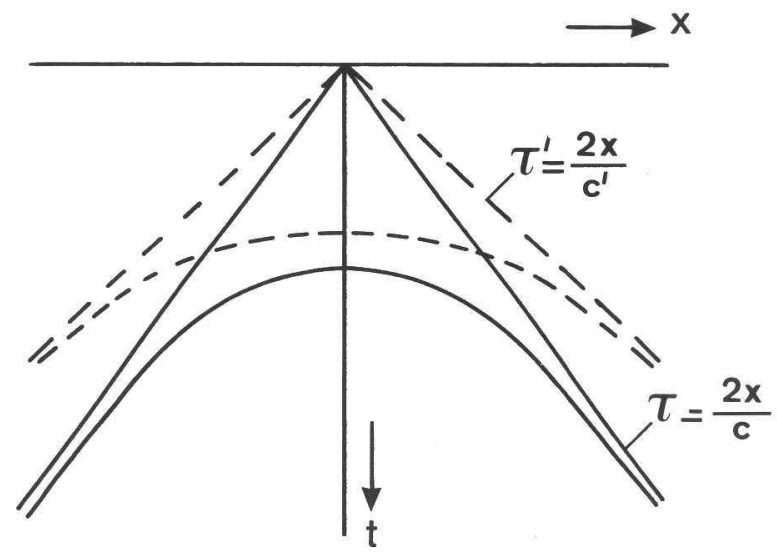

Figure III-4: Trave1 times and trave1 time compensation values: two hyperbolae.

solid curve: travel times $\tau(x), E q \cdot$ (III-7a),

dashed curve: compensation values $\tau^{\prime}(x), E q \cdot($ III-8a).

Eq. (III-6) expresses that application of a matched filter to zero-offset data in the wavenumber-frequency domain corresponds, in the space-time domain, with compensation of travel times, i.e. subtraction from $\tau(x)$ of a value $\tau^{\prime}(x)$ given by the dashed hyperbola in Fig. III-4:

$$
\tau^{\prime}(\mathrm{x})=\frac{2 r}{c^{\prime}}
$$

with asymptotes

$$
\tau^{\prime}= \pm \frac{2 \mathrm{x}}{\mathrm{c}^{\prime}}
$$

In absence of velocity errors $c^{\prime}$ equals $c$, all travel times are fully compensated and the resulting image has optimal lateral resolution determined by spatial bandwidth. If, however, $c^{\prime}$ differs from $c$ the travel times are $n o t$ fully compensated: a fraction $\Delta \tau$ remains, given by

$$
\begin{aligned}
\Delta \tau(x) & =\tau(x)-\tau^{\prime}(x) \\
& =2 r\left(\frac{1}{c}-\frac{1}{c^{\prime}}\right) .
\end{aligned}
$$

Fig. III-5 illustrates how this imperfect travel time compensation leads to deterioration of the imaged result. Fig. III-5a shows the zero-offset response 
of a single dipole diffractor positioned centrally below a line aperture (onesided aperture angle $45^{\circ}$ ). Fig. III-5b shows the diffractor image after matched filtering using correct velocity. Figs. III-5c,d,e,f display the diffractor image after processing with a relative velocity error of $-5 \%,+5 \%$, $-10 \%$ and $+10 \%$ respectively. The upper parts of the figures show the maximum amplitude per trace and can be interpreted as broadband directivity patterns. It is seen that, as in the space-frequency domain, velocity errors lead to lateral d is p e r s $i$ o $n$ of the image in the space-time domain: the image pattern broadens with increasing velocity error whereas the maximum amplitude decreases.

The author has amply discussed, together with a co-author, the effects of velocity errors on imaged results in two papers: De Vries and Berkhout (1983), De Vries and Berkhout (1984), the former of which is added to this thesis as appendix A and should be consulted for details. A few important features are summarized below.

Until now, we only considered inversion procedures, where downward extrapolation takes place to the depth level where the secondary sources are positioned. If the choice of the extrapolation step is free and denoted as $z^{\prime}$ to be distinguished from source depth $z$, the general expression for the travel time fraction which is $n$ o $t$ compensated after extrapolation reads, in paraxial approximation $\mathrm{x}<<\mathrm{z}, \mathrm{z}^{\prime}$ :

$$
\Delta \tau(x)=2\left(\frac{z}{c}-\frac{z^{\prime}}{c^{\prime}}\right)+x^{2}\left(\frac{1}{c z}-\frac{1}{c^{\prime} z^{\prime}}\right),
$$

cf. appendix A, Eq. (3).

Consideration of Eq. (III-10) leads to the following conclusions:

(1) The $f i r s t$ term at the right hand side vanishes if

$$
z^{\prime}=\frac{c^{\prime}}{c} z
$$

Then, the vertical travel time $(x=0)$ is fully compensated and an image is found at or around $t=0$, but localized at a wrong depth. In practice, this situation appears when mapping techniques are applied which only take the extrapolated data at (or around) $t=0$ into account (e.g. Stolt, 1978). 
de
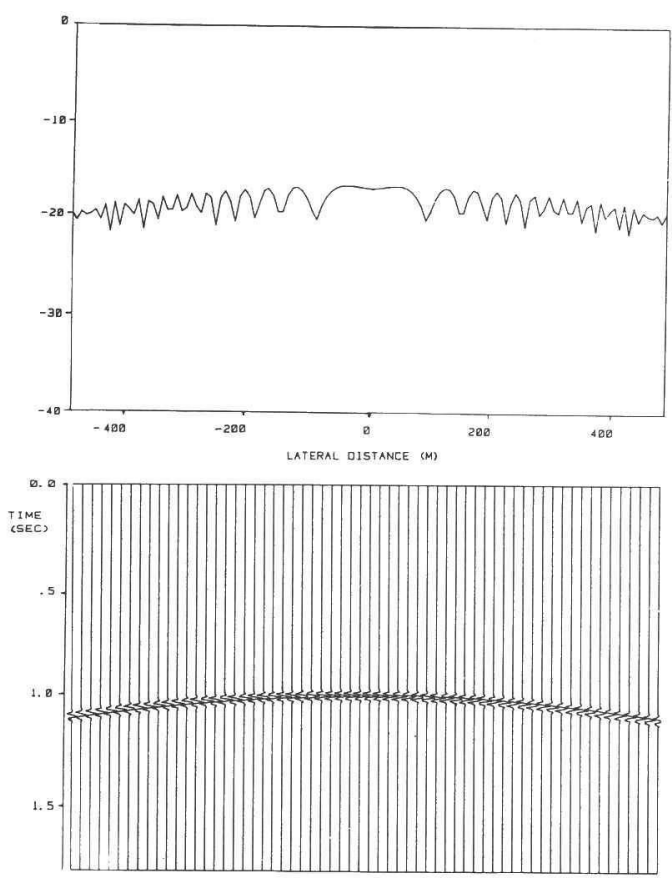

C C
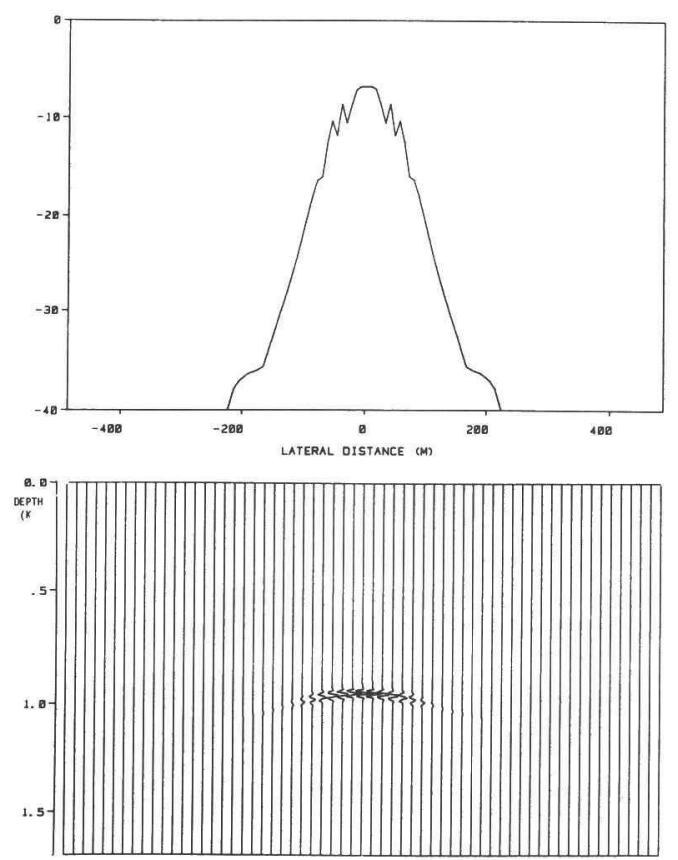
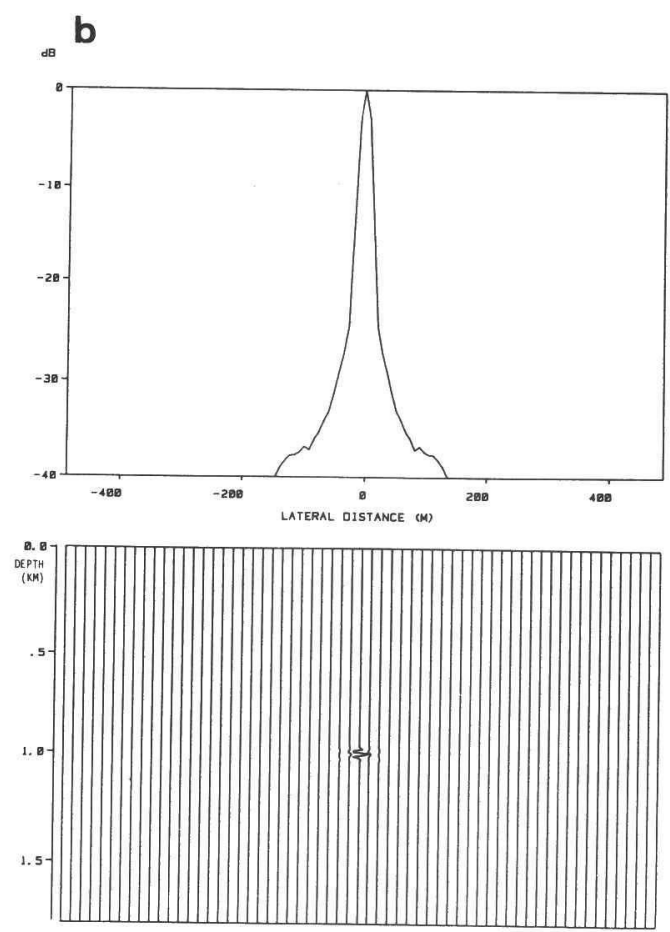

d d
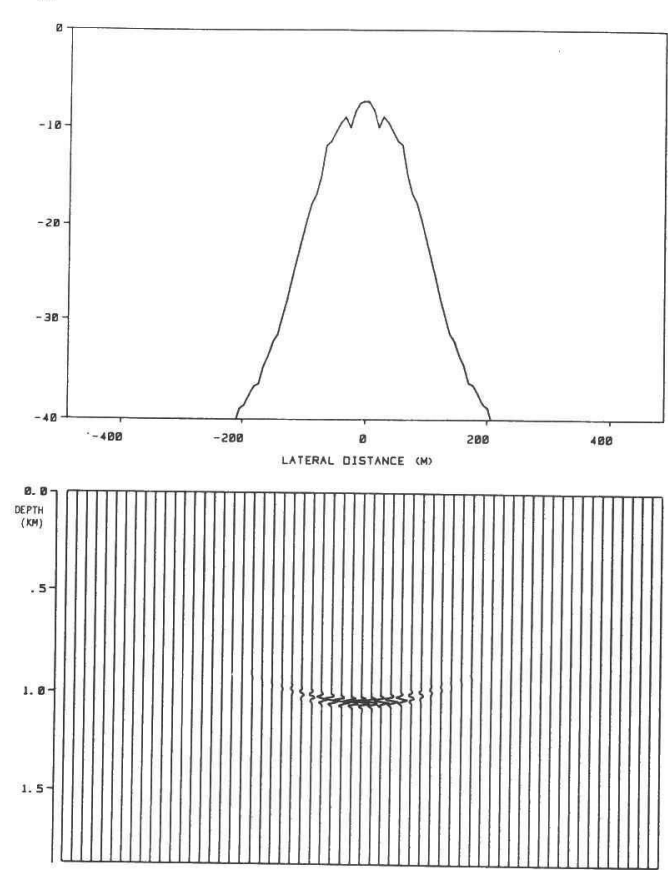

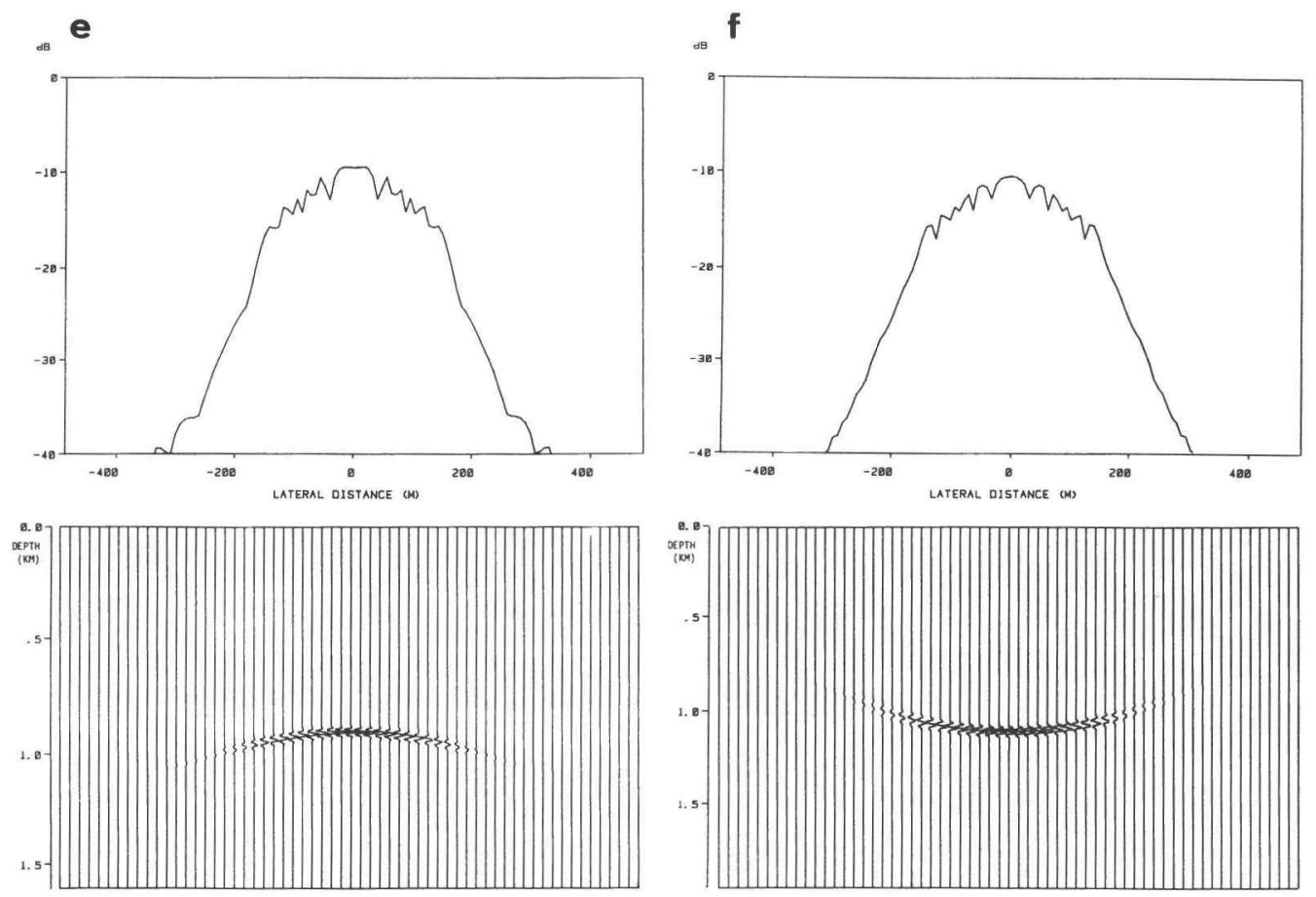

Figure III-5: Influence of velocity errors on the image of a single diffractor (one-sided aperture angle $45^{\circ}$ ):

a: zero-offset response

b: image obtained with correct velocity

c, d: id. with $-5 \%$ and $+5 \%$ relative velocity error

e,f: id. with $-10 \%$ and $+10 \%$ relative velocity error

The upper figure parts show the corresponding directivity patterns. 

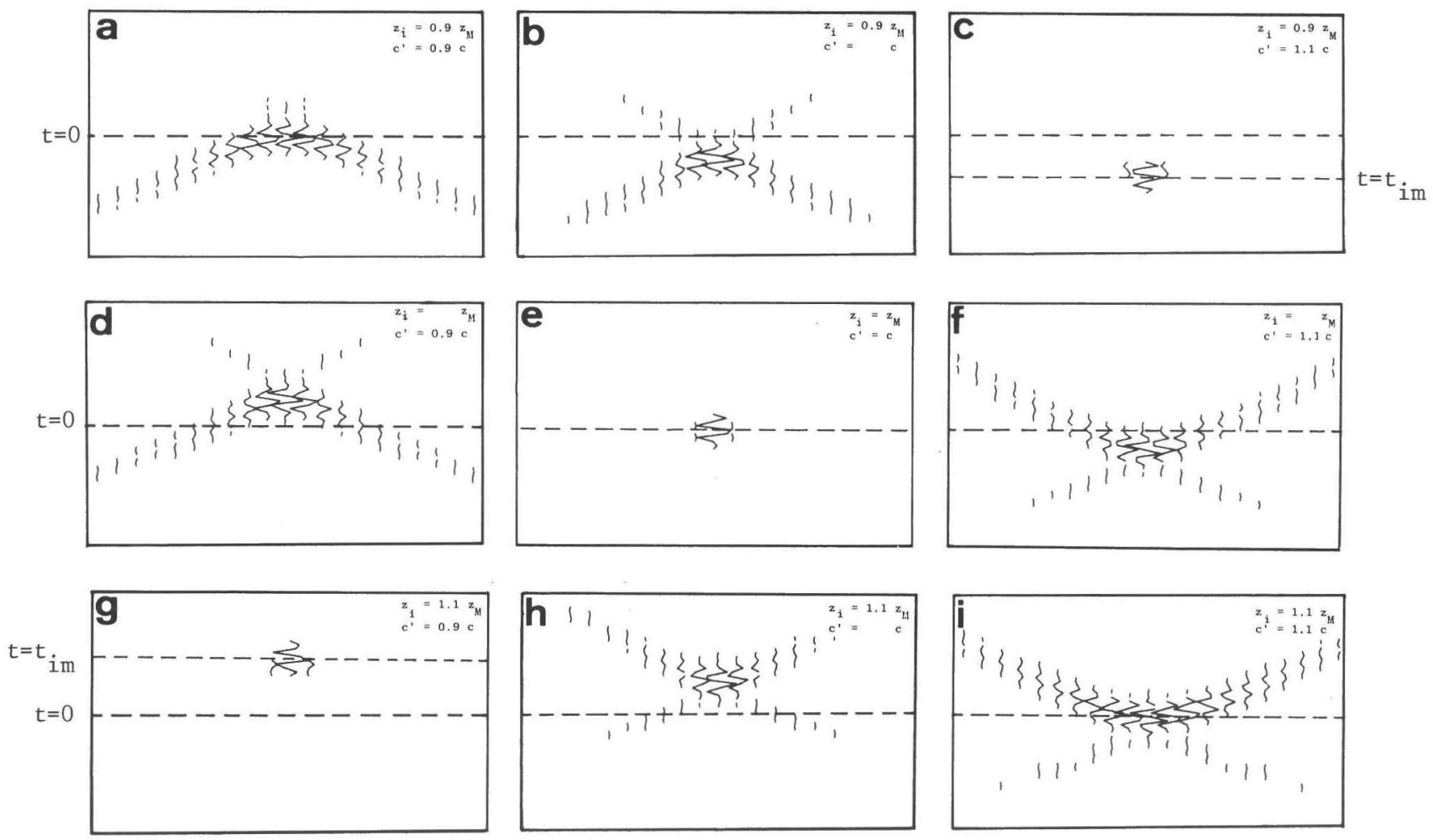

Figure III-6: Inverted response of a dipole diffractor after downward extrapolation to various depth levels with various velocity values. 
(2) The $\mathrm{s}$ e $\mathrm{c}$ o $\mathrm{n} d$ term at the right hand side vanishes if

$$
z^{\prime}=\frac{c}{c^{\prime}} z
$$

Then, the uncompensated travel time fraction $\Delta \tau$ is independent of $\mathrm{x}$, yielding an image with optimal lateral resolution shifted from $t=0$ to

$$
t=t_{i m}=\left[\left(\frac{c^{\prime}}{c}\right)^{2}-1\right] \frac{2 z^{\prime}}{c^{\prime}}
$$

and positioned at an erroneous depth according to Eq. (III-12). Hence, velocity errors are $e x c h$ a $n g$ e d with depth errors to get an optimal image. This image, however, is only obtained if, using a recursive 'stripping' technique, the extrapolated result is time-windowed around $\mathrm{t}=\mathrm{t}_{\mathrm{im}}$ and not, as usual, around $\mathrm{t}=0$. From the value of $\mathrm{t}_{i \mathrm{~m}}$, $\mathrm{c}$ can be determined as was also suggested by Yilmaz and Chambers (1980).

Fig. III-6 illustrates the above phenomena. The inverted response of a dipole diffractor is given for various choices of $z^{\prime}$ and various velocity errors. Special attention should be paid to the following points of interest:

(1) The figures of the middle row $(d, e, f)$ give the inverted response at diffractor depth, $z^{\prime}=z$. It is clearly shown that, apart from time errors, the data is u n d e r migrated ('moustache'-shape) for $c^{\prime}<c$ (d) and o v e r migrated ('smile'-shape) for $c^{\prime}>c(f)$.

(2) The figures on the diagonal $(a, e, i)$ give the images as mapped around $t=0$, i.e. after full compensation of vertical time: $z^{\prime} / c^{\prime}=z / c$. The effects of undermigration for $c^{\prime}<c$ (a) and overmigration for $c^{\prime}>c$ (i) are even stronger than for $z^{\prime}=z$ above.

(3) The figures on the anti-diagonal $(c, e, g)$ show the images resulting after recursive stripping: $z^{\prime}=\left(c / c^{\prime}\right) z$. For all values of $z^{\prime}$, an image with optimal lateral resolution is found around $t=t{ }_{i m}$.

\section{III.4. EXAMPLES}

To show the effects of velocity errors on the imaged results of more complicated structures, the images are given of the same configurations as in section II.4, but now also with a relative velocity error of $-10 \%$ and $+10 \%$. 

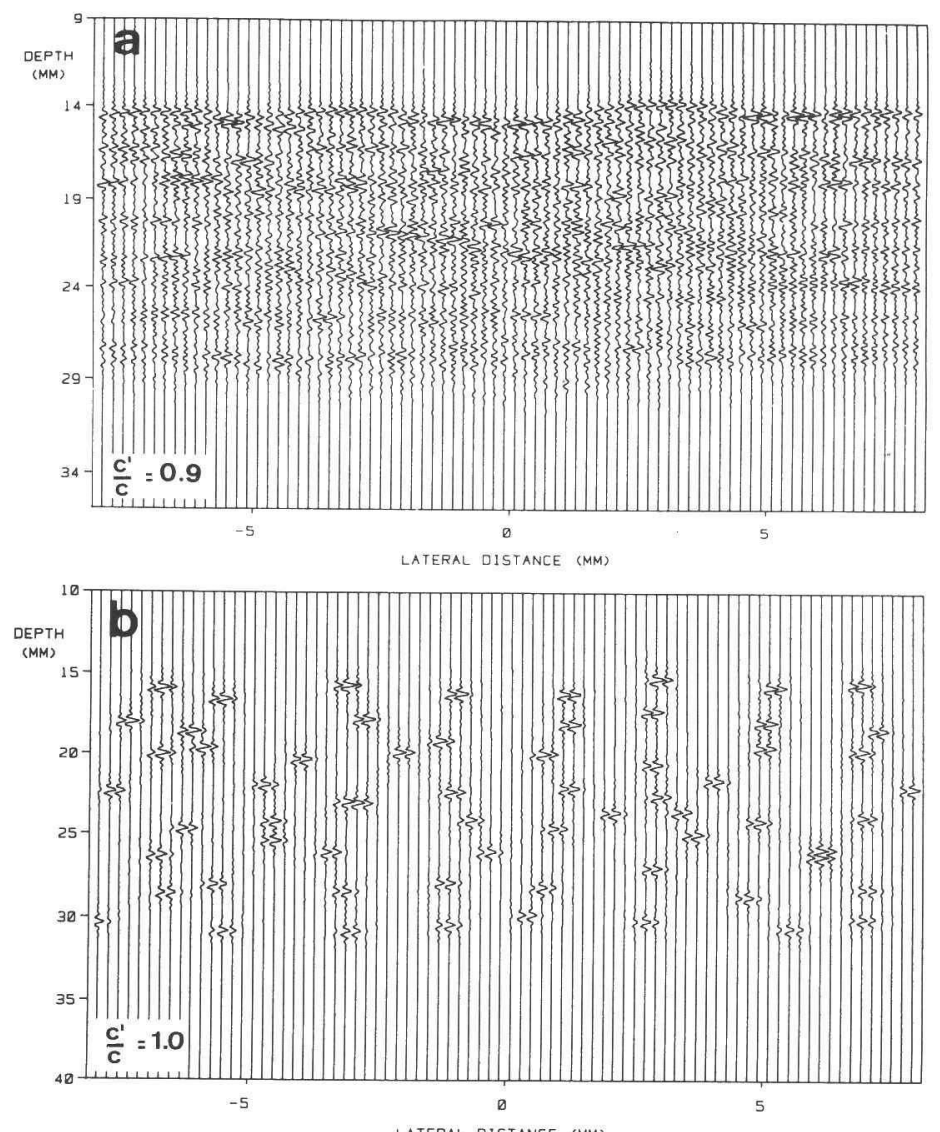

LATERAL DISTANCE (MM)

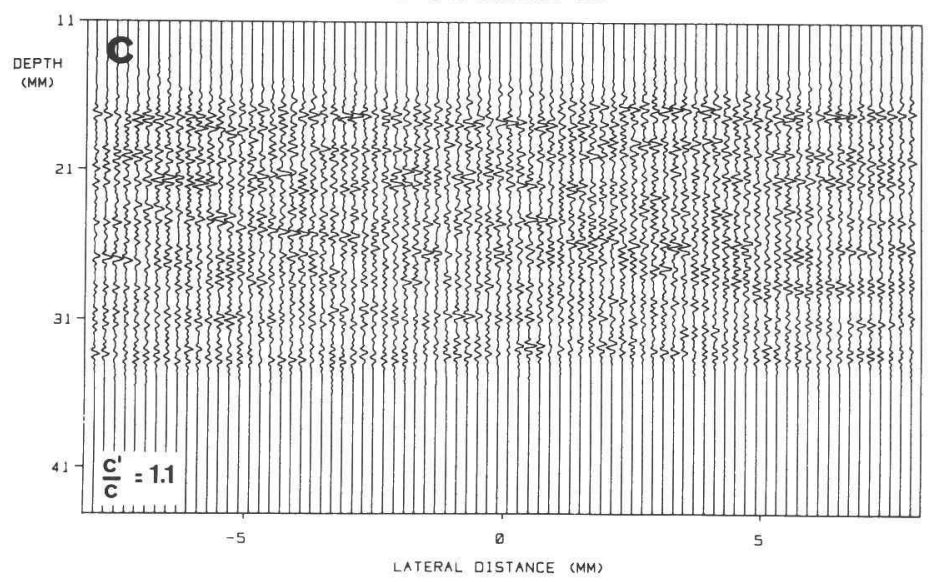

Figure III-7: Image of the diffractor distribution specified in Fig. II-10.

a: $c^{\prime} / c=0.9$

b: $c^{\prime} / c=1.0$

c: $c^{\prime} / c=1.1$ 


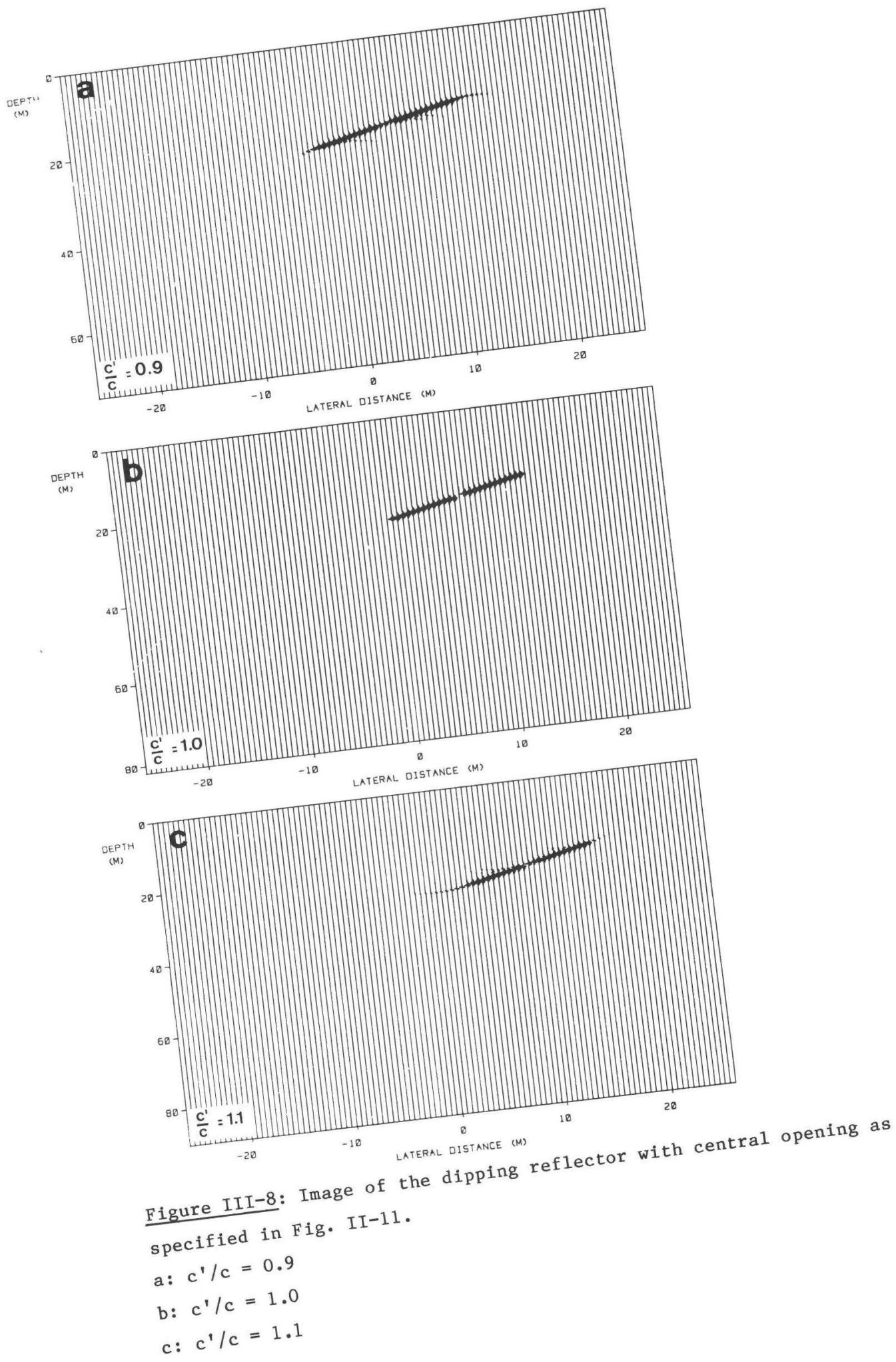


Fig. III-7 shows the mapped image of a 64 point diffractor distribution, Fig. III-8 the mapped image of a dipping reflector with a central opening.

It is seen that velocity errors yield two effects:

(1) deterioration of 1 a $\mathrm{t}$ e $\mathrm{r}$ a $1 \mathrm{r}$ e s $01 \mathrm{u} \mathrm{t} i \circ \mathrm{n}$, especially at places where diff $\mathrm{r}$ a $\mathrm{c} i \circ \mathrm{n}$ energy is generated, i.e. at point diffractors and edges of reflectors,

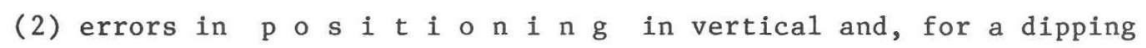
reflector, also in horizontal direction. 


\section{CHAPTER IV:}

\section{ENTROPY}

\section{IV.1 INTRODUCTION}

After showing, in the previous chapters, the importance of velocity information in echo-acoustics, and before showing in the next chapter how so-called minimum entropy norms can be successfully applied for velocity analysis, we shall discuss in this chapter the concept and properties of e $\mathrm{n} t \mathrm{r}$ o $\mathrm{y}$ and, especia11y, mi n i m u m e $\mathrm{m}$ r o p y. After some notes about the history of the entropy idea, the concept of entropy as introduced by Shannon (1948) in communication theory will be exposed. It will be shown how, in a simple and logical way, this entropy can be related to the concept of minimum entropy as found in geophysical 1iterature. On this concept, a generalized minimum entropy (ME) norm is defined which can be interpreted as a measure of resolving power of a single- or multi-trace data set, its value being determined by parameter sparsity and system dispersivity. This norm is compared with ME-norms as appearing in geophysical literature as a tool for deconvolution of seismic recordings - the limitations of which are indicated in this chapter as well. Next, the behaviour of our ME-norm is discussed under variation of echoacoustical data set properties as pulse bandwidth and phase, reflectivity density, interference and noise. To complete this chapter about entropy, an appendix is dedicated to maximum entropy spectral analysis, a well-known signal processing technique which is shown to have little more in common with our ME-norm than the word 'entropy'. 


\section{IV.2 SHANNON'S ENTROPY AND RELATED NORMS}

\section{IV.2.1 Historical notes}

'Entropy', being a more or less established concept in communication and information theory, has for a long time only been known in the field of thermodynamics. There, it was introduced in the 19th century (Clausius, 1875), giving a relation between increment of heat during a reversible process and absolute temperature.

In 1948, Shannon introduced in his already classic paper "A Mathematical Theory of Communication", as a measure of a priori u n c e r t a $i \mathrm{n} y$ about the realization of an event, the function

$$
H=-\sum_{i} p_{i} \log p_{i},
$$

$p_{i}$ being the probability of event $i$. This function in itself was not new: Boltzmann used its continuous analog as early as 1872 , as a probability measure of transitions of gas molecules. The reason for Shannon to call the function 'entropy' seems to have the following anecdotic character, as told by Tribus (1978), who interviewed Shannon about his personal reaction when he realized that he had identified a measure of uncertainty:

Shannon said that he had been puzzled and wondered what to call his function. 'Information' seemed to him to be a good candidate as a name, but 'Information' was already badly overworked. Shannon said he sought the advice of John von Neumann, whose response was direct, "You should call it 'entropy' and for two reasons: first, the function is already in use in thermodynamics under that name; second, and more importantly, most people don't know what entropy really is, and if you use the word 'entropy' in an argument you will win every time!"

Since Shannon gave his measure this name, discussions have been started about the relation, or even the possible identity, between the entropy in statistical thermodynamics on the one side, and the 'new' entropy in communication theory on the other. Brillouin (1953) showed a close relation and consistency, Jaynes (1957) proved a sort of identity. 
The great merit of Shannon is that he supplied the function given in Eq. (IV-1) with a universal meaning, thus making it possible to develop it further and to apply it in many fields of research. Tribus (1978) gives a survey of applications, which comprises diverging topics as land use planning, molecular biology and exploration seismology.

In the next paragraphs, we shall study Shannon's entropy function in some more detail, and see how it is related to maximum and minimum entropy concepts used in (e.g. echo-acoustical) signal processing.

\section{IV.2.2 Shannon's entropy: a measure of a priori uncertainty}

Shannon formulated his 'entropy', in discrete notation, as:

with:

$$
H=-\sum_{i=1}^{N} p_{i} \log p_{i},
$$

$$
\sum_{i=1}^{N} p_{i}=1 \text {, }
$$

where $\mathrm{N}$ is the number of possible events (e.g. results of an experiment) and $p_{i}$ is the probability of event $i$.

Let us consider two extreme situations:

a. Only o n e event is possible, which means that there is no a priori uncertainty, and realization of the event (e.g. execution of the experiment) does not yield any information, where we define 'information' as the difference between a priori and a posteriori uncertainty.

Hence:

$$
\begin{aligned}
& \mathrm{P}_{i}=0, \quad i \neq j \\
& \mathrm{p}_{i}=1, \quad i=j,
\end{aligned}
$$

as illustrated in Fig. IV-1.

Then, since by convention

$$
\lim _{i \rightarrow 0} p_{i} \log p_{i}=0,
$$

we find for the entropy the $\mathrm{m} i \mathrm{n} i \mathrm{~m} \mathrm{u}$ malue:

$$
\mathrm{H}=0 \text {. }
$$




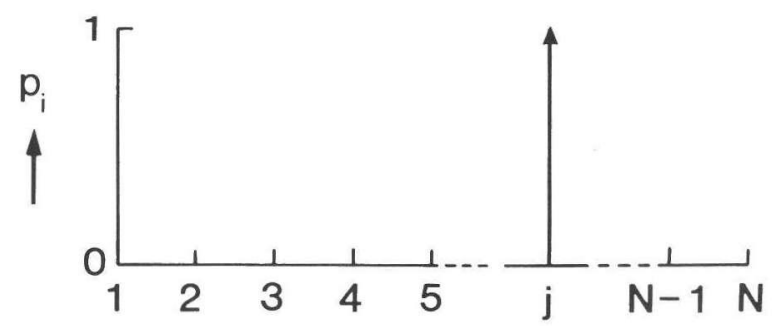

Figure IV-1: Probability distribution for minimum (zero) entropy.

b. Al1 $\mathrm{N}$ events have equal probability, which corresponds with a maximal a priori uncertainty and maximum information by realizing an event. From Eq. (IV-2b) follows for this case:

$$
\mathrm{p}_{\mathrm{i}}=\frac{1}{\mathrm{~N}}, \mathrm{i}=1, \ldots, \mathrm{N} \text {. }
$$

This is illustrated in Fig. IV-2.

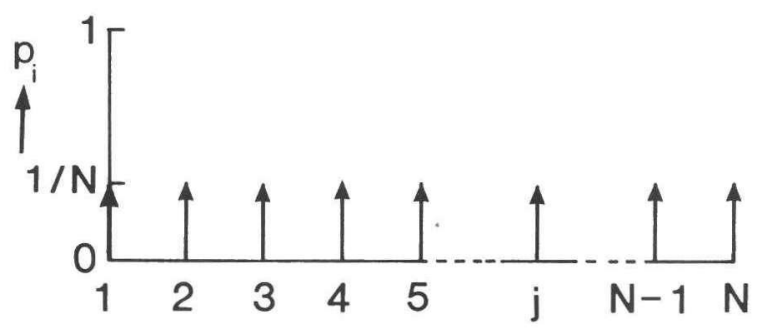

Figure IV-2: Probability density distribution for maximum entropy.

For the entropy we now find:

$$
\begin{aligned}
H & =-N \cdot \frac{1}{N} \log \left(\frac{1}{N}\right) \\
& =\log N
\end{aligned}
$$

which, under the constraint formulated in Eq. (IV-2b), is the $\mathrm{max} i$ m $u$ m value of $\mathrm{H}$. 
We conclude that a basic requirement for Shannon's entropy to be a measure of a priori uncertainty is fulfilled: no uncertainty corresponds with zero entropy, maximum uncertainty corresponds with maximum entropy. It should be noted that more properties are required (such as additivity and symmetry) to give a full characterization of entropy.

\section{IV.2.3 A measure of a priori certainty}

Let us now introduce another function $\mathrm{H}^{\prime}$ of $\mathrm{p}_{i}$, as follows:

$$
H^{\prime}=\frac{1}{N} \sum_{i=1}^{N}\left(N p_{i}\right) \log \left(N p_{i}\right) \text {, }
$$

with, according to Eq. (IV-2b):

$$
\sum_{i=1}^{N} N p_{i}=N \sum_{i=1}^{N} p_{i}=N .
$$

It is easily seen that there is a simple relation between $\mathrm{H}^{\prime}$ and $\mathrm{H}$ :

$$
\mathrm{H}^{\prime}=\log \mathrm{N}-\mathrm{H} \text {. }
$$

When entropy $\mathrm{H}$ equals zero, $\mathrm{H}^{\prime}$ has its maximum value $\log \mathrm{N}$, and vice versa. Since $\mathrm{H}$ is a measure of a priori uncertainty, $\mathrm{H}^{\prime}$ can be used as a criterion of a priori c e $r$ t a $i \mathrm{n} t$.

Table IV/1 shows values of $H$ and $H^{\prime}$ for some simple probability distributions with $N=8$, which number has also been chosen as the base of the logarithm.

It should be noted that:

(1) each probability distribution uniquely defines a value of $\mathrm{H}$ and a value of $\mathrm{H}^{\prime}$,

(2) $\mathrm{H}$ and $\mathrm{H}^{\prime}$ are $\mathrm{s} y \mathrm{~mm}$ e $t \mathrm{r}$ i c measures: the order of the probabilities has no influence on the values of $H_{\text {H }}$ and $H^{\prime}$ (cf. Table IV/l, c and $d, g$ and h),

(3) a sparse and peaked distribution yields a low value of $\mathrm{H}$ and a high value of $\mathrm{H}^{\prime}$,

(4) a dense and smooth distribution yields a high value of $\mathrm{H}$ and a low value of $\mathrm{H}^{\prime}$. 
Tab1e IV/1

Uncertainty and certainty measures for some simple probability distributions. (Only the values $0, .1, .5$ and 1 are represented in the distributions).

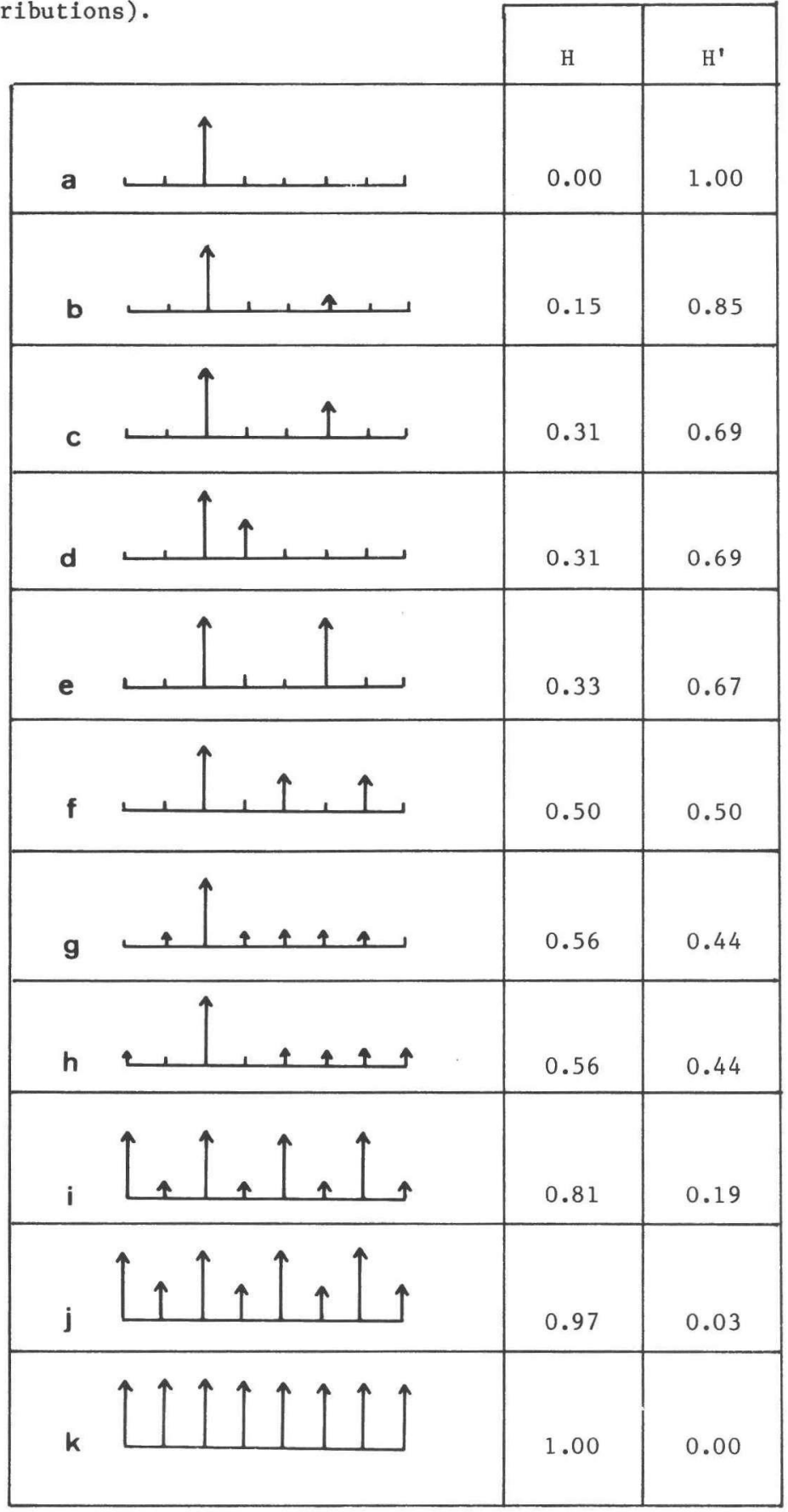




\section{IV.2.4 Entropy of an amplitude distribution: a measure of resolving power}

We now leave the field of statistics with its probability distributions, and consider instead an N-point data set described by some positive definite parameter $a_{i}$, such as:

- the absolute value of the data

- the squared value (i.e. a measure for the local energy)

- the RMS value

- the envelope

For simplicity we call $a_{i}$ the 'amplitude' of the data. We normalize the amplitudes to the sum of the amplitude distribution:

$$
a_{i}^{\prime}=\frac{a_{i}}{\sum_{i} a_{i}},
$$

and define:

$$
q_{i}=N a_{i}^{\prime}=\frac{a_{i}}{\frac{1}{N} \sum_{i} a_{i}}=\frac{a_{i}}{\overline{a_{i}}},
$$

so that $q_{i}$ is a scale-independent version of amplitude $a_{i}$.

Note that:

$$
\sum_{i=1}^{N} a_{i}^{\prime}=1
$$

and

$$
\sum_{i=1}^{N} q_{i}=N \text {, }
$$

which is entirely analogous to Eqs. (IV-2b) and (IV-8b) for probability $p_{i}$ and product $\mathrm{Np}_{i}$ respectively.

This means that a function $V$, defined as:

$$
v=\frac{1}{N} \sum_{i=1}^{N} q_{i} \log q_{i},
$$

is related to the normalized amplitude distribution - or, apart from a constant, to the amplitude distribution itself - in exactly the same way as certainty measure $\mathrm{H}^{\prime}, \mathrm{Eq}$. ( $\left.\mathrm{IV}-8 \mathrm{a}\right)$, is related to the corresponding probability 
density distribution ${ }^{1}$. Hence, a sparse and peaked amplitude distribution yields a high value of function $V$, a dense and smooth distribution a low value.

As in subsection IV.2.2, we now consider two extreme situations:

a. All amplitudes are zero, except one which has value A.

Hence:

$$
\begin{aligned}
& a_{i}=0, i \neq j \\
& a_{i}=A, i=j
\end{aligned}
$$

and:

$$
\begin{aligned}
& a^{\prime}=0, i \neq j \\
& a^{\prime}=1, i=j .
\end{aligned}
$$

This amplitude distribution is illustrated in Fig. IV-3.

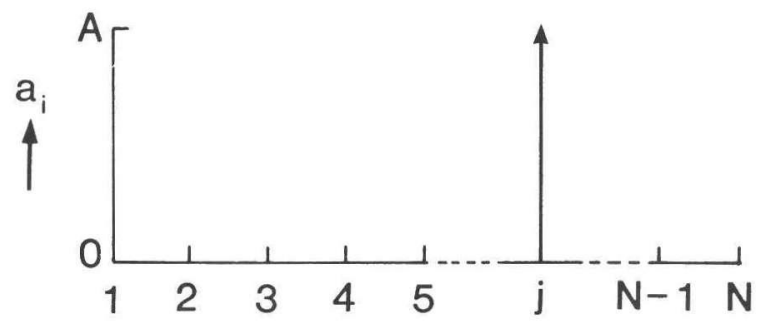

Figure IV-3: Amplitude distribution with minimum (zero) entropy.

Due to the complete analogy with Eq. (IV-3) and Fig. IV-1, it is obvious to call this amplitude distribution, with $\mathrm{V}=\log \mathrm{N}$, a m $\mathrm{i} \mathrm{n} \mathrm{im} \mathrm{u}$ (or: zero) e $\mathrm{n} t \mathrm{r} \circ \mathrm{p} y$ amplitude distribution. In geophysical literature dealing with deconvolution of seismic data sets, e.g. Wiggins (1978), this nomenclature is used indeed, but with a rather vague argumentation instead of the straightforward connection shown here.

1) The 'parsimonious norm' P as defined by Claerbout (1977) is the analog of entropy H, Eq. (IV-2), for such an amplitude distribution:

$$
P=-\sum_{i=1}^{N} a_{i}^{\prime} \log a_{i}^{\prime}
$$

See also the footnote on page 83 . 
b. All amplitudes have equal value A.

Hence:

$$
a_{i}=A, i=1, \ldots, N
$$

and:

$$
a_{i}^{\prime}=\frac{1}{N}, i=1, \ldots, N \text {, }
$$

which yields the discribution shown in Fig. IV-4.

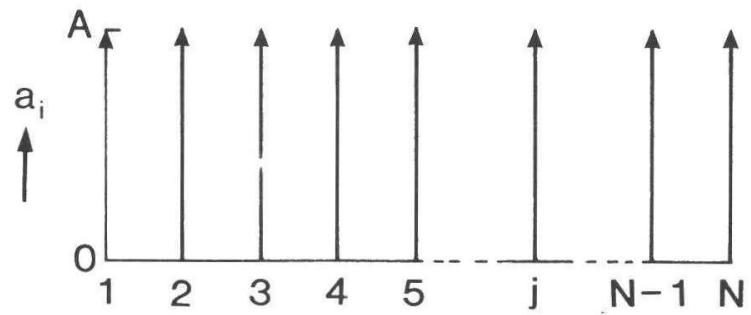

Figure IV-4: Amplitude distribution with maximum entropy

Based on the complete analogy with Eq. (IV-6) and Fig. IV-2, we call this distribution, with $\mathrm{V}=0$, a maximum entropy amplitude distribution.

In echo-acoustical practice, one-dimensional amplitude distributions as discussed above are often dealt with in the form of time traces describing, for the detector position considered, the response of a system to some source wavelet. The $s$ p a $r$ i $t$ y of such a trace is determined by the sparsity of the reflections in the system. The ' $c \circ \mathrm{m} \mathrm{a} \mathrm{c} \mathrm{t} \mathrm{n} \mathrm{e} \mathrm{s} \mathrm{s'} \mathrm{of} \mathrm{the} \mathrm{wavelet} \mathrm{after}$ travelling through the medium from source to detector determines whether a reflection appears peaked or smoothed in the trace pattern. Compactness is interpreted here as a quality inverse to $\mathrm{d} i \mathrm{~s}$ e $\mathrm{r} s \mathrm{i} \mathrm{n}$, being determined by properties of both the source and the medium of propagation. Reflection sparsity and wavelet compactness together describe the $\mathrm{r}$ e s o 1 $v$ i n $g$ p o w e $r$ of the trace:

high sparsity and high compactness (= low dispersion) yield a trace with a few high amp1itude peakes and, hence, high resolving power,

low sparsity and low compactness (= high dispersion) yield a trace with a 'smeared out' amplitude distribution and, hence, low resolving power. 
Generalizing the analogy mentioned above, we conclude that the entropy of an echo-acoustical data trace decreases with increasing resolving power, i.e. with increasing reflector sparsity and increasing wavelet compactness. Function V, defined in Eq. (IV-12a), i n creases with d e creasing entropy, and will therefore in the following be called a M i n $i \mathrm{~m} \mathrm{~m}$ E $\mathrm{n} t \mathrm{r}$ o $\mathrm{p}$ n $\mathrm{orm}$.

\section{IV.2.5 Entropy of an amplitude distribution related to uncertainty}

The interesting question arises if the entropy of an echo-acoustical trace, as defined in the previous subsection, has any relation with 'uncertainty', for which the original entropy as defined by Shannon is a criterion.

The answer is positive as far as we consider the uncertainty in the $i \mathrm{n} e \mathrm{r}-$ $p r e t a t i \circ n$ of the data. When the entropy is high, i.e. the amplitude distribution is smooth, we cannot decide if we have sparse reflections convolved with a dispersed wavelet, or dense reflections convolved with a compact pulse. This means high uncertainty in interpretation. On the other hand, when the entropy is low, i.e. the trace shows a few high amplitude peaks, interpretation can be done with low uncertainty.

We conclude that the entropy of an amplitude distribution, although defined on grounds of formal analogy, is also related to the entropy as defined by Shannon in terms of interpretation.

\section{IV.3 MINIMUM ENTROPY NORMS}

\section{IV.3.1 Influence of amplitude parameter $\mathbf{a}_{\mathbf{i}}$}

According to the conventions of the previous section we apply to an N-point data trace $y_{i}(i=1,2, \ldots, N)$, a minimum entropy $(M E)$ norm ${ }^{1}$ ) of the form:

$$
\mathrm{V}=\frac{1}{\mathrm{~N}} \sum_{i} \mathrm{q}_{\mathbf{i}} \log \mathrm{q}_{\mathbf{i}}
$$

with

$$
q_{i}=\frac{a_{i}}{\frac{1}{N} \sum_{i} a_{i}} \text {, }
$$

1) 'norm' is used here as in common language, not in topological sense. 
$a_{i}$ being a positive definite amplitude parameter of data $y_{i}$. Note that $y_{i}$ is 'mapped' in an unambiguous way into data $a_{i}$. It was seen that this norm $V$ can be interpreted as a measure of the resolving power of the data trace, since it yields a high value if the trace contains a few high peaks.

The actual values of $\mathrm{V}$, and the sensitivity for variations in $\mathrm{y}_{i}$, are evidently influenced by the choice of amplitude parameter $a_{i}$. The optimal choice of $a_{i}$ depends on the application considered.

In table IV/2, values of $\mathrm{V}$ (with log-base 8) are given for some simple 8-point data distributions and different choices of $a_{i}$. To enhance comparability, the values of $\mathrm{V}$ are normalized, for all choices of $a_{i}$, to 1.00 for the minimum entropy configuration (a). The following amplitude parameters are considered:

$$
\begin{aligned}
a_{i} & =\left|y_{i}\right|, \\
a_{i} & =a_{i, \exp }=1-\exp \left(-\frac{\left|y_{i}\right|}{\left|y_{i}\right|_{\max }}\right) \\
& =\frac{\left|y_{i}\right|}{\left|y_{i}\right| \max }-\frac{1}{2}\left(\frac{\left|y_{i}\right|}{\left|y_{i}\right|_{\max }}\right)^{2}+0\left\{\left(\frac{\left|y_{i}\right|}{\left|y_{i}\right|_{\max }}\right)^{3}\right\}, \\
a_{i} & =y_{i}^{2}, \\
a_{i} & =\operatorname{env}_{\left(y_{i}\right),} \\
a_{i} & =\operatorname{env}^{2}\left(y_{i}\right) .
\end{aligned}
$$

Here, env $\left(y_{i}\right)$ denotes the envelope of the data, defined as:

$$
\operatorname{env}\left(y_{i}\right)=\left[y_{i}^{2}+H^{2}\left\{y_{i}\right\}\right]^{\frac{1}{2}}
$$

$\mathrm{H}\left\{\mathrm{y}_{\mathbf{i}}\right\}$ being the Hilbert transform of $\mathrm{y}_{i}$. It should be noted that for $a_{i}=e n v\left(y_{i}\right)$, ME-norm $V$ is not symmetric in the sense of note (2) on page 67. Hence, in this case the analogy with Shannon's entropy concept is violated on a fundamental point. 
Table IV/2

ME-norm $V=\overline{q_{i} \log _{8}\left(q_{i}\right)}$ for different choices of $a_{i}=\bar{a}_{i} q_{i}$. (The distributions of $y_{i}$ contain values $0, .1, .5$ and 1 and are identical to the probability distributions in Table IV/1.)

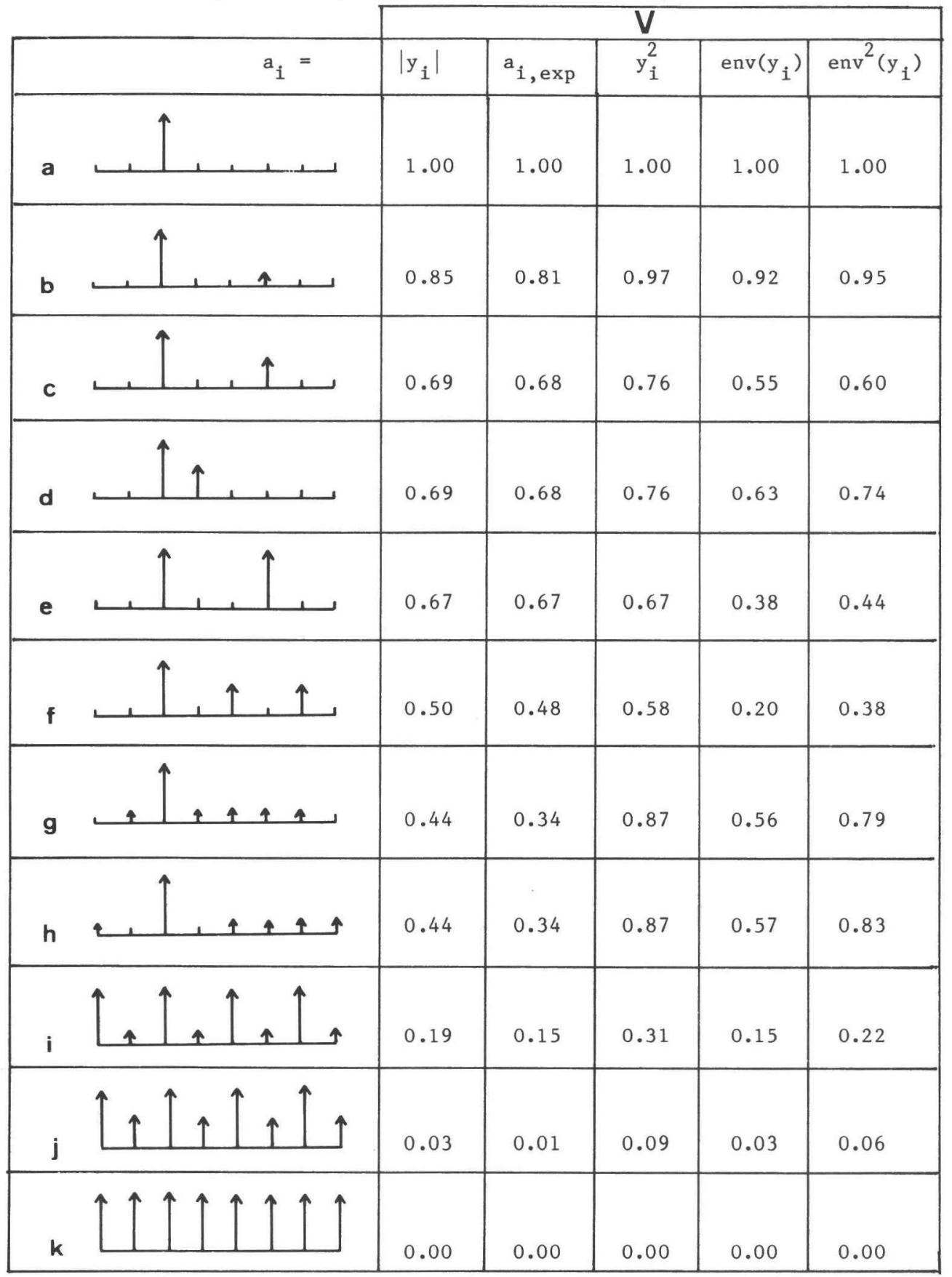


The following features are noteworthy:

(1) For amplitude parameter choice $a_{i}=\left|y_{i}\right|, V$ is very sensitive to the presence of additive small values (e.g. noise), as is seen by comparison of the values for configurations $a, b$ and $g$, or $i$ and $j$. For $a_{i}=$ $1-\exp \left[-\left|y_{i}\right| /\left|y_{i_{2}}\right|_{\max }\right]$ this effect is even stronger. On the contrary, the choice $a_{i}=y_{i}^{2}$ makes $V$ less sensitive to small values. Which choice is preferable depends on the application considered: if sma11 amplitudes must be detected, a high sensitivity of $\mathrm{V}$ to such amplitudes is desired; if influence of noise must be eliminated, $V$ should be chosen insensitive to lower amplitude values.

(2) The e n v e $10 \mathrm{p}$ e of a data configuration not only depends on the data values but also on their order. Therefore, in contrast with the other amplitude parameters, for $a_{i}=\operatorname{env}\left(y_{i}\right)$ and $a_{i}=e n v^{2}\left(y_{i}\right), v$ has different values for configurations $c$ and $d$, also for $g$ and $h$. In this way an ME-norm can discriminate between data sets with equal amplitude distributions but different intervals.

\section{IV.3.2 Generalization of the minimum entropy norm concept}

If some norm has to be a measure of resolving power of a data trace $y_{i}$, a necessary condition is that it emphasizes peaks in the amplitude distribution of $y_{i}$. If the amplitude is represented by a function $a_{i}$ as described above, a peak corresponds with a value of $a_{i}$ exceeding strongly the average value $\overline{a_{i}}$ and hence with a large value of $q_{i}=a_{i} / \overline{a_{i}}$. Emphasizing high values of $q_{i}$ is realized by multiplying $q_{i}$ with a weighting function $F\left(q_{i}\right)$ increasing monotonically with $q_{i}$.

In the ME-norm defined by Eq. (IV-15), this weighting function is given by

$$
F\left(q_{i}\right)=\log q_{i} \text {, }
$$

which indeed increases monotonically, but slowly, with $q_{i}$. To have a more flexible measure of resolving power that can be specified in dependence of the application considered, we generalize the norm $V$ given above to:

$$
\mathrm{V}=\frac{1}{\mathrm{~N}} \sum_{i} \mathrm{q}_{\mathrm{i}} \mathrm{F}\left(\mathrm{q}_{\mathrm{i}}\right)
$$

where the only requirement to function $\mathrm{F}\left(\mathrm{q}_{i}\right)$ is that it increases monotonically with $q_{i}$. 
Notes:

(1) For the generalized form of Eq. (IV-18), the full formal analogy between $\mathrm{V}$ and the entropy-related certainty measure $H^{\prime}$ given in Eq. (IV-8) no longer holds. Still, we shall persevere in calling $V$ a minimum entropy (ME) norm, according to the analogy in interpretation discussed in subsection IV.2.5. Moreover, we shall denote weighting function $F\left(q_{i}\right)$ as e $n t r \circ p y$ f u n c t $i \circ n$.

(2) For entropy functions other than $F\left(q_{i}\right)=\log q_{i}$, the values of $v$ are not restricted to the interval between zero and $\log \mathrm{N}$ as before. For, e.g., $F\left(q_{i}\right)=q_{i}^{p}$, the minimum value of $V$ is 1 and the maximum value is ${ }^{p}$. Still, the extreme values are obtained for the same data distributions as before.

An entropy function $F\left(q_{i}\right)$ increasing weakly with $q_{i}$ yields an ME-norm in which also the lower data values - i.e. the amplitude distribution below the peaks play a significant role. On the other hand, a function $F\left(q_{i}\right)$ increasing strongly with $q_{i}$ leads to an ME-norm, the value of which is determined by a few peaks only. To illustrate this, table IV/3 shows values of ME-norms $V$ as defined by Eq. (IV-18), for some simple 8-point data distributions, with the following choices of $F\left(q_{i}\right)$ :

$$
\begin{aligned}
& F\left(q_{i}\right)=\log _{8}\left(q_{i}\right), \\
& F\left(q_{i}\right)=q_{i}^{\frac{1}{2}}, \\
& F\left(q_{i}\right)=q_{i}, \\
& F\left(q_{i}\right)=q_{i}^{2}, \\
& F\left(q_{i}\right)=q_{i}^{4} .
\end{aligned}
$$

To eliminate the influence of the amplitude parameter $a_{i}$, this parameter has been chosen $\left|y_{i}\right|$ and the average value $\overline{a_{i}}$ has been taken equal for all situations. Then, the configurations considered represent the distributions of $y_{i}$ as well as $a_{i}$ and $q_{i}$. In order to enhance the comparability, again the values of $\mathrm{V}$ are normalized to 1.00 for the minimum entropy configuration (a). It is seen that the spread of lower amplitude values (cf. configurations $f, g$, h) strongly influences $V$ in case of 'weak' entropy functions as $\log q_{i}$ or $q_{i}^{\frac{1}{2}}$, whereas $V$ is invariant to such differences for $F\left(q_{i}\right)=q^{4}$. For the latter entropy function, $V$ is very sensitive to the number of high peaks: cf. configurations a, b, c. 
Table IV/3

ME-norm $V=\overline{q_{i} F\left(q_{i}\right)}$ for different choices of entropy function $\mathrm{F}\left(\mathrm{q}_{i}\right)$.

The data sets represent the distributions of (positive) variable $y_{i}$ as we11 as $a_{i}$ and $q_{i}$, since $a_{i}=\left|y_{i}\right|$ and $\bar{a}_{i}$ is constant.

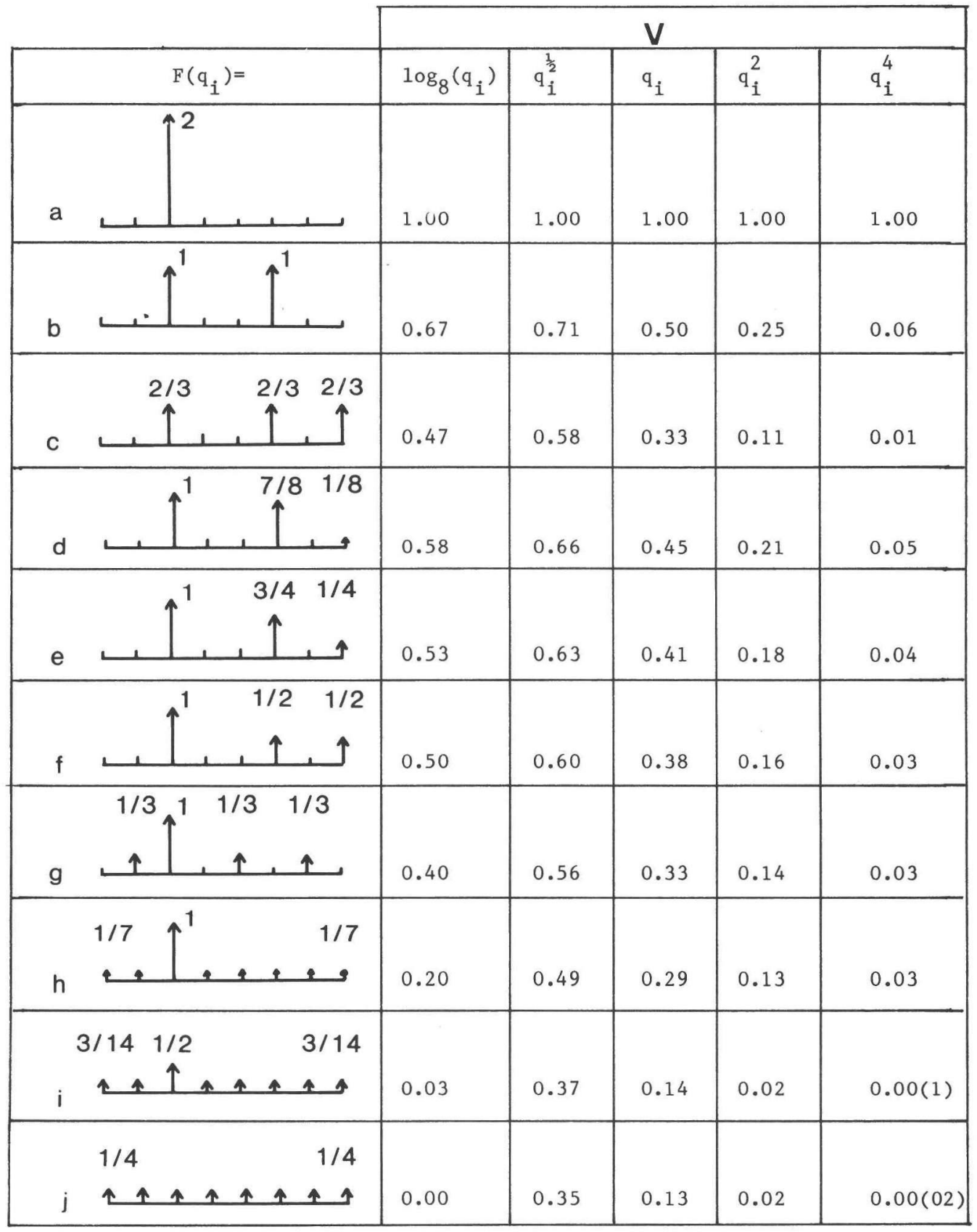


In echo-acoustics, a data set usually consists of some gather of related data traces. Moreover, the main goal of inversion techniques is to produce a wellresolved image of reflectors and diffractors, where noise and propagation effects are eliminated as much as possible. Therefore, it seems convenient to define a measure of resolving power for a multi-trace data set, to which the ME-norm defined by Eq. (IV-18) can be an appropriate basis. As a m u $1 \mathrm{t} i$ $\mathrm{t} \mathrm{r}$ a $\mathrm{c}$ e ME-norm for a data set comprising $M$ traces we introduce:

$$
V=\sum_{j=1}^{M} w_{j} V_{j} .
$$

$\mathrm{V}_{j}$ is the contribution of the $j$-th data trace, defined by Eq. (IV-18) and now written as:

$$
v_{j}=\frac{1}{N} \sum_{i=1}^{N} q_{j i} F\left(q_{j i}\right) .
$$

${ }^{w}{ }_{j}$ is a $t r a c e$ w e $i g h t$ i $g$ f a $c t o r$, giving the opportunity to enlarge the contribution of significant traces and suppress the influence of traces containing only insignificant data values or noise. (Note that the value of $\mathrm{V}_{j}$ is scale-independent, so that without weighting the contribution to $\mathrm{V}$ by traces containing significant and insignificant data would be of equal importance!)

In order to make the contribution to $\mathrm{V}$ of an individual trace proportional to its 'strength', the trace weighting factors $w_{j}$ should take into account the ratio of some 'strength' parameter per trace to the total value of that parameter calculated over the whole multi-trace data set. In table IV/4, the values of ME-norm $V$ according to Eq. (IV-19) are given, applied to the data sets of Fig. IV-5, for $a_{i}=y_{i}^{2}, F\left(q_{i}\right)=q_{i}$, and the following choices of $w_{j}$ : 


$$
\begin{aligned}
& \left.\mathrm{w}_{\mathrm{j}}=\mathrm{w}_{\text {unwgh }}=1 / \mathrm{M} \text { ( } \mathrm{M} \text { is number of traces }\right) \text {, } \\
& w_{j}=w_{\max , 1 i n}=\frac{\left|y_{j i}\right|_{\max , j}}{\sum_{j}\left|y_{j i}\right|_{\max , j}}, \\
& w_{j}=w_{\max , s q u}=\frac{\left(y_{j i}^{2}\right)_{\max , j}}{\sum_{j}\left(y_{j i}^{2}\right)_{\max , j}}, \\
& w_{j}=w_{\text {sum, } 1 \text { in }}=\frac{\sum_{i}\left|y_{j i}\right|}{\sum_{j} \sum_{i}\left|y_{j i}\right|} \text {, } \\
& w_{j}=w_{\text {sum, squ }}=\frac{\sum_{i} y_{j i}^{2}}{\sum_{j} \sum_{i} y_{j i}^{2}} .
\end{aligned}
$$

To enhance comparability, the maximum value of $V$ is normalized to 1.00 .

\section{$\underline{\text { Table IV } / 4}$}

Multi-trace ME-norm for the data sets of Fig. IV-5, for different trace weighting factors.

amplitude parameter: $a_{i}=\bar{a}_{i} q_{i}=y_{i}^{2}$,

entropy function : $F\left(q_{i}\right)=q_{i}$.

$\mathrm{V}_{\mathrm{a}}, \mathrm{V}_{\mathrm{b}}$ denote the ME-norm values obtained for the data sets of

Fig. IV-5a and $-5 b$ respectively.

\begin{tabular}{|l|c|c|}
\hline${ }_{j}$ & $v_{a}$ & $v_{b}$ \\
\hline$w_{\text {unwgh }}$ & 0.62 & 0.07 \\
$w_{\text {max }}$ 1in & 0.95 & 0.30 \\
${ }_{\text {max, squ }}$ & 1.00 & 0.57 \\
${ }_{\text {w }}$ sum, 1in & 0.88 & 0.09 \\
w $_{\text {sum, squ }}$ & 1.00 & 0.30 \\
\hline
\end{tabular}



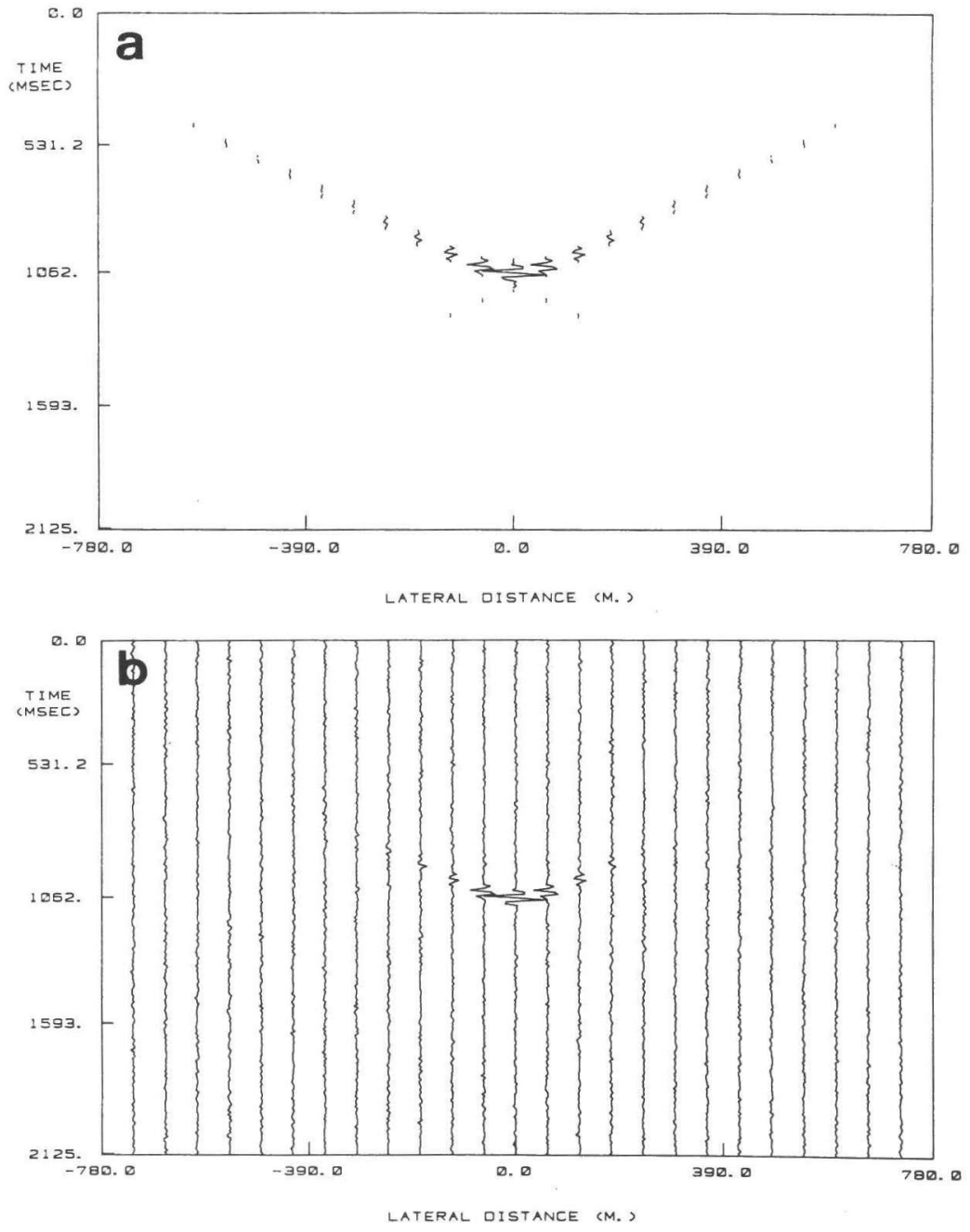

Figure IV-5: Two multi-trace data sets:

a: without noise

b: with noise, S/N-ratio $10 \mathrm{~dB}$ 
The following points should be noted:

(1) For the noise-free data set (Fig. IV-5a) most traces contain one wavelet. Since ME-norm $V_{j}$ is independent of the scale of the data, the values will be of the same order for all traces except the practically empty ones. Therefore, the value of multi-trace ME-norm V is of the same order for all trace weighting factor choices, see the $\mathrm{V}_{\mathrm{a}}$-column in tabel IV/4. Only for averaging without weighting, i.e. $w_{j}=1 / M$, when also the empty traces where $V_{j} \approx 0$ are fully taken into account, the value of $V$ is significantly lower.

(2) In the data set of Fig. IV-5b, the weaker wavelets are masked by noise. All traces except a few ones around the center now have a very low $\mathrm{V}_{j}-\mathrm{value}$, so that for any trace weighting factor $V$ is significantly lower than in the noise-free case.

(3) For the noisy data set, the presence in a trace of one wavelet rising significantly above the noise will have a relatively small influence on the $\mathrm{s} u \mathrm{~m}$ of $|\mathrm{y}|$ or $\mathrm{y}^{2}$ in that trace. If it is desirable to suppress the influence of the presence of noise it is preferable to use a trace weighting factor $w_{t}$ based on the $m$ a $x i m u m$ per trace of $|y|$ or, even better, $y^{2}$; see the $V_{b}$-column in table IV/4. Doing so, however, the danger arises that an 'occasional' high noise peak significantly influences the resulting $\mathrm{ME}-$ norm value.

\section{IV.3.3 Minimum entropy norms in literature}

Since the late seventies, measures of 'spikiness' or 'ordering' of a data set based on the minimum entropy concept appear in geophysical literature, to be used as a criterion for optimal deconvolution of a seismic registration: Minimum Entropy Deconvolution (MED), see Wiggins (1978), Ooe and U1rych (1979), Deeming (1981). In those papers, the relation between minimum entropy and the 'entropy' as defined by Shannon - see subsection IV.2.2 - is rather vague: if, anyhow, an argumentation about the terminology is given, it usually follows the line that 'entropy' is a kind of synonym to 'chaos', 'disorder', so that maximal ordering should correspond with minimum entropy. In fact, the word 'entropy' could entirely be missed in this context. Since, however, it is used after a11 and a relationship with the entropy concept as used in communication and information theory has to be indicated, the author prefers the argumentation given in subsections IV.2.4 and IV.2.5: a formal identity of the basic formulae, supported by common aspects of 'uncertainty'. 
In the following, we shall give a survey of ME-norms found in geophysical literature. Many of those norms are based on optimization criteria used in more general - i.e. not specifically seismic - data analysis procedures, where they were not associated with any 'entropy'-idea. The ME-norms discussed are not necessarily of the form we introduced in Eq. (IV-18), but they all have in common that they are non-linear functions of the amplitude of the data, emphasizing significant peaks.

Wiggins (1978) uses, as a criterion for optimal deconvolution of a seismic trace, the v a r i m a x -norm introduced by Kaiser (1958) as a tool for maximization of the variance of a set of orthogonal vectors, and evaluated by Cooley and Lohnes (1971):

$$
v_{j}^{\operatorname{varim}}=\frac{\sum_{i} y_{j i}^{4}}{\left(\sum_{i} y_{j i}^{2}\right)^{2}}
$$

which fits into our definition apart from the factor $\frac{1}{\mathrm{~N}}$ :

$$
v_{j}^{\text {varim }}=\sum_{i} q_{j i} F\left(q_{j i}\right),
$$

with:

$$
\begin{aligned}
& a_{j i}=y_{j i}^{2}, \\
& q_{j i}=\frac{y_{j i}^{2}}{y_{j i}^{2}}, \\
& F\left(q_{j i}\right)=q_{j i} .
\end{aligned}
$$

A multi-trace version of the varimax-norm with a trace-independent normalization factor is the $\mathrm{k} \mathrm{u} \mathrm{r} \mathrm{t} \mathrm{o} \mathrm{s} \mathrm{i} \mathrm{s} \mathrm{-norm,} \mathrm{introduced} \mathrm{by} \mathrm{Saunders} \mathrm{(1953,}$ 1961) and used for seismic deconvolution by Ooe and Ulrych (1979):

$$
v^{k u r}=\frac{\sum_{j} \sum_{i} y_{j i}^{4}}{\left(\sum_{j} \sum_{i} y_{j i}^{2}\right)^{2}}
$$


In order to get an ME-norm which, in MED-performance, yields better detection of small reflections than the varimax-and kurtosis-norms, claerbout (1977) 1 ) developed his e $x \mathrm{t}$ i n s i $\mathrm{M}$ E p o w e $r$ n o $r \mathrm{~m}$ :

$$
v_{j}^{e \cdot p}=\sum_{i} y_{j i}^{2} \log y_{j i}^{2}-\left(\sum_{i} y_{j i}^{2}\right) \log \frac{1}{N} \sum_{i} y_{j i}^{2},
$$

which takes values between zero and $\log \mathrm{N}$, just as our ME-norm with logarithmic weighting function, Eq. (IV-12). The norm is called 'extrinsic' because its value is additive in the sense that

$$
v_{j+k}^{e \cdot p}=v_{j}^{e \cdot p}+v_{k}^{e \cdot p}
$$

Another way to enlarge the detectability of small reflections is indicated by Ooe and Ulrych (1979): they modified the varimax- and kurtosis-norms by replacing the amplitude parameter $a_{j i}=y_{j i}^{2}$ by:

$$
a_{j i}=1-\exp \left[-\alpha \frac{y_{j i}^{2}}{\left(y_{j i}^{2}\right)_{\max , j}}\right] \text {, }
$$

where $\alpha$ is a positive constant to be chosen appropriately. For $\alpha \rightarrow 0$, the original varimax- and kurtosis-norms reappear.

1) In this reference, Claerbout also introduces his p a $r$ s $i$ o $n$ i $u s$ $\mathrm{n} \circ \mathrm{rm}$, which is not an ME-norm, but a norm increasing with entropy:

$$
P_{j}=\log \sum_{i}\left|y_{j i}\right|^{n}-\frac{\sum_{i}\left|y_{j i}\right|^{n} \log \left|y_{j i}\right|^{n}}{\sum_{i}\left|y_{j i}\right|^{n}} \quad(n>0)
$$

Its value is between zero (for minimum entropy as in Fig. IV-3) and $10 \mathrm{~g}$ (for maximum entropy as in Fig. IV-4). Note that for $a_{j i}=\left|y_{j i}\right|^{n}$ and $\sum_{i} a_{j i}=1$ this norm reads:

$$
P_{j}=-\sum_{i} a_{j i} \log a_{j i}
$$

which is fully analogous to Shannon's entropy H, Eq. (IV-2). 
In his interesting survey on minimum entropy deconvolution, Deeming (1981) considers the same generalized ME-norm formula that we gave in Eq. (IV-18):

$$
v_{j}=\frac{1}{N} \sum_{i} q_{j i} F\left(q_{j i}\right)
$$

with

$$
q_{j i}=\frac{a_{j i}}{\overline{a_{j i}}},
$$

but only considers the amplitude parameter

$$
a_{j i}=y_{j i}^{2}
$$

\section{IV.4 SENSITIVITY OF MINIMUM ENTROPY NORMS TO VARIATIONS IN ELEMENTARY DATA TRACE PROPERTIES}

We have seen that ME-norms are a measure of resolving power of an echoacoustical data set, which is determined by two main factors:

(1) the $\mathrm{c} \circ \mathrm{mpa} \mathrm{ct} \mathrm{nes}$ of the wavelet with which the reflections are convolved, determined by $d i s p$ e $s i o n$ effects due to source and medium of propagation. Compactness is quantified by $\mathrm{b}$ a $\mathrm{n} \mathrm{d} \mathrm{w} \mathrm{d} d \mathrm{t}$ h and $\mathrm{p} h$ a $\mathrm{s}$ e spectrum of the wavelet;

(2) the s p a r s i t y or, inversely, the density of reflections.

Consequently, the value of an ME-norm of a data set will be significantly influenced by variations in those elementary parameters. Moreover, interference between adjacent wavelets may occur, also affecting the value of ME-norm $V$. Measured data are always more or less polluted with coherent and/or incoherent $\mathrm{n}$ o i s e ; in other words: some distribution of detrimental amplitudes is added to the useful data, thus disturbing the information of interest and correspondingly diminishing the value of ME-norm $V$.

Quantitatively, the influence on $\mathrm{V}$ of the effects mentioned above will depend on the choice of amplitude parameter $a_{i}$, entropy function $F\left(q_{i}\right)$ and trace weighting factor $w_{j}$. Qualitatively, however, the trends are the same for any choice of these norm parameters. Therefore, we shall discuss the sensitivity of $V$ to the above effects only for amplitude parameter $a=y^{2}$ and a few choices of 


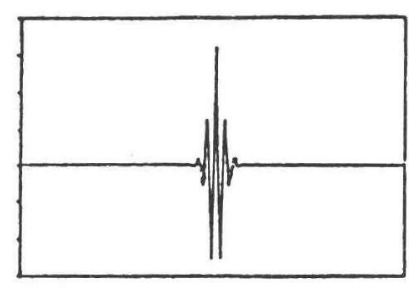

$s_{0}(t)$
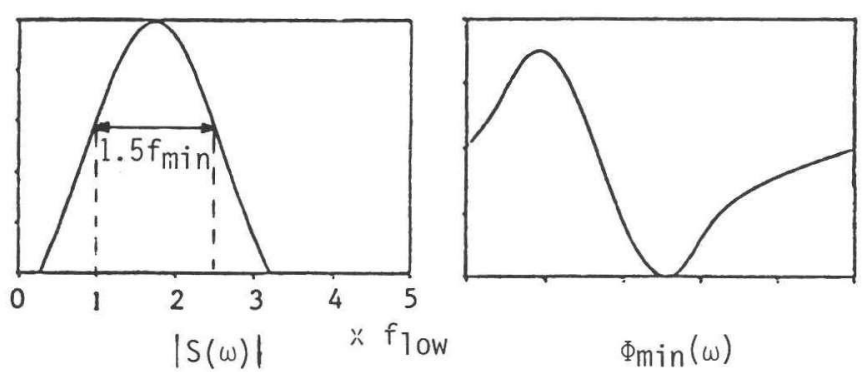

$\Phi_{\min }(\omega)$

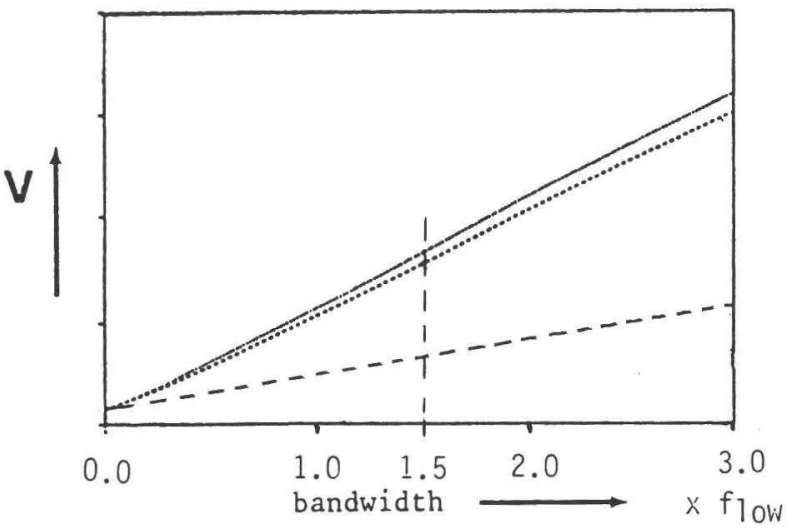

Figure IV-6: Influence of bandwidth and phase on ME-norm V. smá11 figures:

wavelets $s(t)$ with identical amplitude spectrum $|S(\omega)|$ and phase spectra $\Phi(\omega)$ which are zero, minimum and mixed respectively.

1arge figure:

ME-norm $V$ as a function of bandwidth

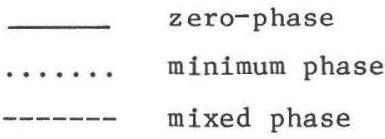

amplitude parameter: $a=y^{2}$.

entropy function : $F(q)=q$.

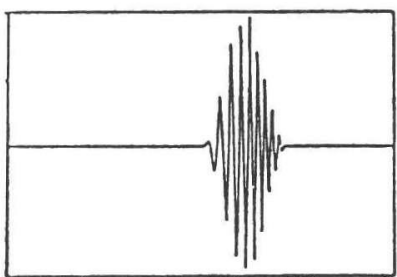

$\mathrm{s}_{\text {mixed }}(\mathrm{t})$

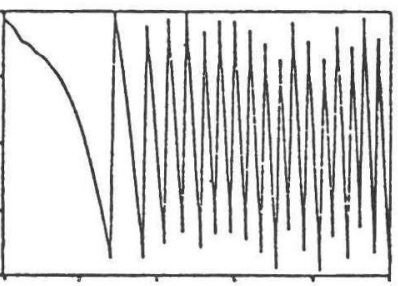

$\Phi_{\text {mixed }}(\omega)$ 
$F(q)$, omitting the subscripts $i$ for notation simplicity. Moreover, we consider one single data trace.

In Fig. IV-6 the effects on $V$ of the wavelet compactness parameters, i.e. b a n d w i d $t h$ and $p h$ a $s$, are illustrated for $F(q)=q \cdot V$ is calculated for a data trace containing only one wavelet, as a function of the bandwidth of this wavelet. For each bandwidth, three phase spectra are considered, corresponding with zero-phase, minimum-phase and mixed-phase wavelet character. It is seen that $\mathrm{V}$ increases monotonically with increasing bandwidth (i.e. increasing compactness and decreasing length), the slope being largest for zero-phase and smallest for mixed-phase wavelets. For one bandwidth value, the Hanningwindowed amplitude spectrum, the phase spectra and the corresponding time wavelets are displayed as well. The well-known fact that, for a given amplitude spectrum, the zero-phase spectrum corresponds with the shortest possible wavelet length (e.g. Berkhout, 1974), is clearly illustrated. The general conclusion from Fig. IV-6 is that ME-norm $V$ i n creases with i $n$ creasing wavelet compactness, i.e. d e creasing dispersion.

The dashed curves in Fig. IV-7 show, for $F(q)=\log _{e}(q), q^{\frac{1}{2}}, q$ and $q^{2}$ respectively, the values of $\mathrm{V}$ as a function of reflection $\mathrm{d} e \mathrm{n} s \mathrm{i} t \mathrm{y} d$, i.e. the percentage of points filled with unit dirac pulses in a trace containing for the rest only zero's. As expected, $V$ decreases monotonically with $d$, with a decay rate depending on the entropy function $\mathrm{F}(\mathrm{q})$. The solid curves in Fig. IV-7 also give $V$ as a function of $d$, but now after convolution of the unit dirac pulses with a zero-phase wavelet of bandwidth $4 \mathrm{f}_{\min }(\mathrm{e} . \mathrm{g} .: 10-50 \mathrm{~Hz}$, or $1-5 \mathrm{MHz}$ ). Since in this case $\mathrm{i} n \mathrm{t}$ e $\mathrm{r}$ f e $\mathrm{r}$ e $\mathrm{n} \mathrm{e}$ between neighbouring wavelets may occur, affecting the value of $\mathrm{V}$, for each value of $\mathrm{d}$ the result is given after averaging over 50 random pulse distributions. It is seen that now $\mathrm{V}$ decreases stronger with $d$ than in the previous broadband situation, for all entropy functions $F(q)$. Practically, one could say that there is a critical density value $d_{\text {crit }}$, depending on $F(q)$, above which the $v$-curve proceeds nearly horizontally - here, some appropriate slope criterion should be introduced - so that for reflection densities higher than $d_{\text {crit }}$ the ME-norm cannot give any indication about the real density value d. Within this limitation, we may draw from Fig. IV-7 the general and expected conclusion that ME-norm V d e creases with i n creasing reflection density, i.e. d e creasing reflection sparsity. 

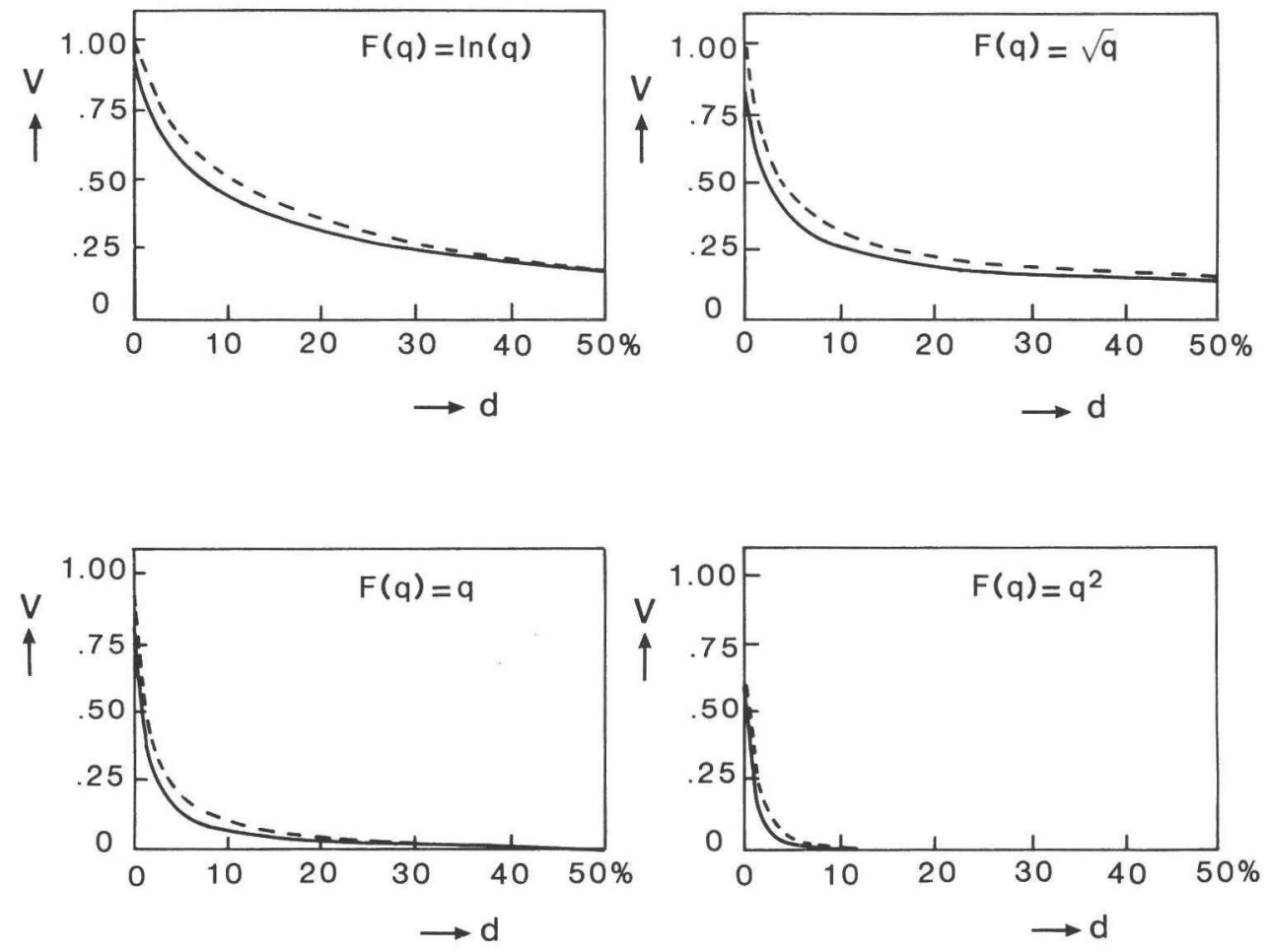

Figure IV-7: ME-norm V as a function of data density d, for different entropy functions $F(q)$.

- - unit dirac pulses, broadband;

: unit dirac pulses, convolved with a zero-phase wavelet of bandwidth $4 \mathrm{f}_{\min }$.

To eliminate fluctuations due to wavelet interference in the band-limited situation (solid curves), for each value of $d$ the result has been averaged over 50 random pulse distributions.

It is interesting to mention here another criterion in data analysis techniques: the so-called WT-criterion, stating that the maximum number of independent parameters in a data recording that can be determined equals the product of bandwidth and registration time. For the band-limited data considered in Fig. IV-7 W=0.4 $\mathrm{f}_{\mathrm{S}}$ ( $\mathrm{f}_{\mathrm{S}}$ is sample frequency) and $\mathrm{T}=256 / \mathrm{f}_{\mathrm{S}}$, so that $\mathrm{WT}=102.4$, corresponding with a density of $40 \%$. For this density value the ME-norm curves in Fig. IV-7 have zero slope. Hence, in terms of the WT-criterion, ME-norms as considered here have a weak discriminating power. This clearly indicates the 
fundamental limitations of ME-norms as a tool for deconvolution of seismic traces, as applied by Wiggins (1978) and other authors mentioned in section IV.3.

The role of $i \mathrm{n} t$ e $\mathrm{r}$ e $\mathrm{r}$ e $\mathrm{n} c \mathrm{e}$ between wavelets in a band-1imited data trace was already mentioned above. To get some insight into these interference effects, the following experiment was done.

A set of 80 dirac-pulses was generated with amplitude values uniformly distributed between -0.5 and +0.5 . This set was randomly placed in a 1024-point data trace - note the low density: $d \approx 7 \%$, so that we have a really sparse data configuration - and then convolved with a band-1imited zero-phase wavelet $\left(W=3 f_{\min }=0.3 f_{s}\right)$. For the same set of 80 dirac-pulses this procedure was repeated 100 times, so that we got 100 data traces with the same parameters but different interference effects. Fig. IV-8 shows the first 20 pulses, before and after convolution, of one realization. For these 100 data traces, ME-norm V was calculated for $a=y^{2}$ and $F(q)=q$, normalized to 1.00 for the mean value. This
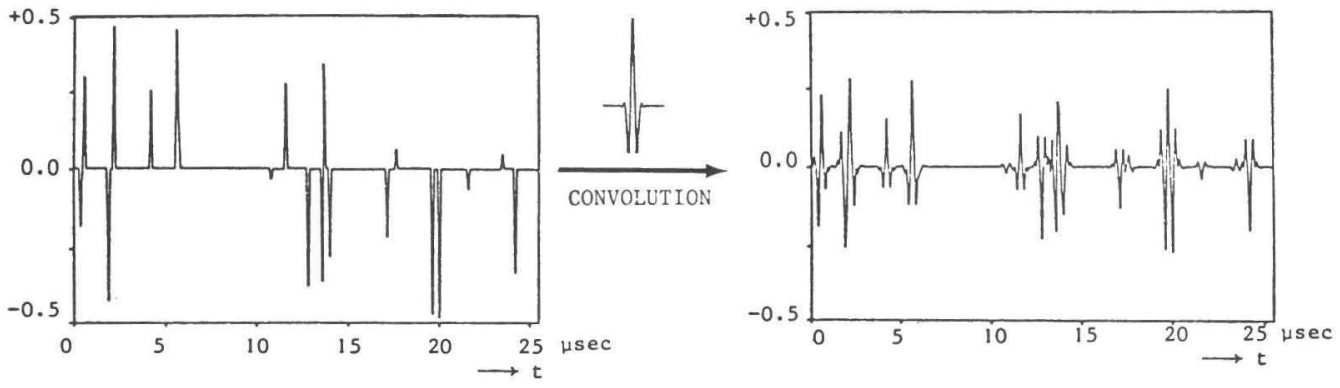

Figure IV-8: Realization of a trace containing 20 zero-phase wavelets with uniformly distributed amplitudes between [ $-0.5,+0.5]$ and random place in the trace. tracelength: 256 points wavelet : bandwidth $1-4 \mathrm{MHz}$, two-sided cosine window $80 \%$. 
mean value $\bar{V}$ and standard deviation $\sigma$ are given in the first row of table IV/5. Then, all traces were split up into two parts of 512 points. For the resulting 200 traces, ME-norm V was calculated, again normalized on the mean value of the

$\underline{\text { Table IV } / 5}$

Normalized mean and standard deviation of ME-norm $V$ for sets of band-1imited data traces of different lengths.

\begin{tabular}{|c|c|c|c|}
\hline tracelength & number of traces & mean value $\overline{\mathrm{V}}$ & standard deviation $\sigma$ \\
\hline \multirow{2}{*}{1024} & 100 & 1.00 & 0.07 \\
512 & 200 & 1.01 & 0.15 \\
256 & 400 & 1.03 & 0.28 \\
128 & 800 & 1.06 & 0.44 \\
\hline
\end{tabular}

origina1 100 traces. Mean value and standard deviation are given in the second row of table IV/5. The splitting procedure was repeated two more times, the corresponding results complete table IV/5. We see that mean value $\bar{V}$ slowly increases with decreasing trace length, whereas standard deviation $\sigma$ increases much stronger. This means that - as intuitively expected - interference effects cause larger fluctuations in ME-norm $V$ for short traces than for longer ones, since in long traces the effects of interference tend to cancel out. It should be noted, however, that the fluctuations in $V$ are not only due to interference, but to a truncation effect as well: in general, splitting up a trace into two parts of equal length does not yield equal division of the number of pulses.

Finally, we consider the influence of the presence of $n \circ i s$ on the value of $\mathrm{V}$. We take into account incoherent (white) noise as well as coherent noise having the same amplitude spectrum as the signal and appearing as 'grass' in impulse response recordings. As a signal, we use a sparse unit dirac-pulse trace (density 10\%) convolved with a zero-phase wavelet with varying bandwidth. As in Fig. IV-6, $V$ is calculated (here for $a=y^{2}$ and $F(q)=q$ on $1 y$ ) as a function of bandwidth, now averaged over 10 random realizations of the sparse pulse configuration and in the presence of noise. The results are given in Fig. IV-9 for incoherent (a) and coherent (b) noise and different signal-to-noise ratios. Comparing the noise-free results (S/N-ratio $\infty$ ) to the zero-phase curve of Fig. IV-6, we clearly see the effects of interference. Comparing Figs. IV-9a and $b$, we see that the influence of coherent and incoherent noise on $V$ is not 


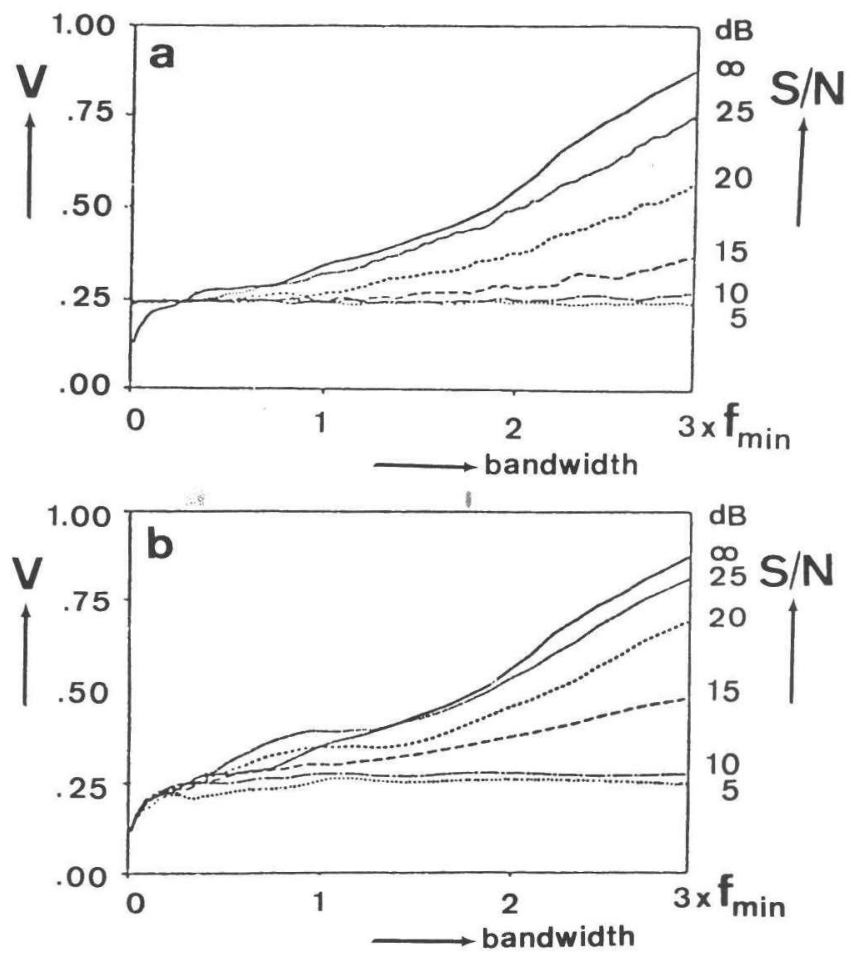

Figure IV-9: ME-norm V calculated as a function of bandwidth, using different signal-to-noise ratios.

a: incoherent noise

b: coherent noise

The zero-phase wavelet is convolved with a sparse trace of unit dirac pulses (density: 10\%). Results are averaged over 10 realizations. amplitude parameter: $a=y^{2}$ entropy function : $F(q)=q$

significantly different. In both cases, the increase of $\mathrm{V}$ with increasing bandwidth (i.e. decreasing wavelet dispersion) is detrimentally affected by increase of noise level; for $\mathrm{S} / \mathrm{N}-\mathrm{ratio} \leq 10 \mathrm{~dB}$, the influence of wavelet dispersion even totally disappears. In general we conclude that the presence of noise diminishes the discriminating power of ME-norms. 


\section{IV.5 MAXIMUM ENTROPY SPECTRAL ANALYSIS}

In the previous sections of this chapter we discussed minimum entropy norms to be used as measures of resolving power of a data set. Obviously related, and more current in literature about data analysis, is the concept of $\mathrm{m}$ a $i$ $\mathrm{m} \mathrm{u}$ m entropy, commonly used in the context of spectral analysis techniques. This maximum entropy concept, however, concerns probability density distributions of (stochastic) data and $\mathrm{n} \circ \mathrm{t}$ amplitude distributions. It is therefore directly dealing with entropy as defined by Shannon, Eq. (IV-2), and has very little to do with the minimum entropy norms we considered above. Merely in order to make this chapter on entropy more complete, we discuss the principles of Maximum Entropy Spectral Analysis (MESA) in appendix B.

\section{IV.6 CONCLUSIONS}

In this chapter norms of the form

$$
v=\frac{1}{N} \sum_{i} q_{i} F\left(q_{i}\right)
$$

have been introduced which quantify the resolving power of a data set. As in geophysical literature, these norms are denoted as m i n i m u m e n t r $\mathrm{p} y$ (ME) norms. It was shown that a justification of this name is found in the analogy, in formulation as well as in interpretation, with the entropy concept known from communication theory.

It was shown how, for a given reflection sparsity, ME-norms are a measure of wavelet dispersion, being determined by bandwidth and phase spectrum. On the other hand, for a given wavelet dispersion, ME-norms are a measure of reflection sparsity.

Final1y, it was shown that decreasing bandwidth (leading to increasing wavelet interference), decreasing sparsity and the presence of noise generally deteriorate the discriminating power of ME-norms, giving a fundamental limitation to their applicability in minimum entropy deconvolution techniques as proposed in the literature. 


\section{CHAPTER V:}

\section{MINIMUM ENTROPY IN RELATION TO VELOCITY ANALYSIS}

\section{V.1 INTRODUCTION}

In chapter III, it was shown that velocity errors lead to lateral dispersion of the spatial wavelets which represent inverted zero-offset data in the spacefrequency domain. In chapter IV, minimum entropy (ME) norms were introduced in the space-time domain, as a measure of resolving power or, for a given sparsity, as a measure of dispersion of a data set. In this chapter, these elements will be brought together. First it is shown that the concept of ME-norms as a measure of resolving power also holds in the space-frequency domain. Hence, correct velocity in the inversion process, corresponding with minimal spatial dispersion of the inverted data, yields maximum values of ME-norms applied to the inverted data in the space-frequency domain. This leads to introduction of 'Minimum Entropy Velocity Analysis' (MEVA), valid for inverted zero-offset data within certain limits of diffractor sparsity in lateral and vertical directions. It will be shown that MEVA applies to data sets other than zero-offset as well, and may also be used in the space-time domain after data transposition.

\section{V.2 APPLICABILITY OF MINIMUM ENTROPY NORMS IN THE SPACE-FREQUENCY DOMAIN}

In chapter IV we applied ME-norms to data sets in the space-time domain as a measure of resolving power along the time axis. It was seen that this resolving power is determined by the sparsity of the parameters together with the compactness of the convolving temporal wavelet. The compactness may be interpreted as an inverse measure of the dispersion of the wavelet determined by bandwidth and phase. 
More specifically, one trace $p(t)$ of a o $n$ e -dimensional pressure recording can be written as:

$$
\begin{aligned}
p(t) & =\sum_{m} a_{m}^{\prime}\left(t-\tau_{m}\right) * r_{m}(t)+n(t) \\
& =s(t) * \sum_{m} a_{m}\left(t-\tau_{m}\right) * r_{m}(t)+n(t),
\end{aligned}
$$

where:

$s(t) \quad$ is the temporal source wavelet,

$r_{m}(t) \quad$ is the reflectivity of the $m^{t h}$ boundary,

$a_{m}\left(t-\tau_{m}\right)$ is the attenuation filter, representing absorption and transmission losses between the surface and the $\mathrm{m}^{\text {th }}$ boundary,

$\tau_{m} \quad$ is the two-way travel time between the surface and the $\mathrm{m}^{\text {th }}$ boundary,

$\mathrm{n}(\mathrm{t}) \quad$ is the additive noise.

After Fourier transformation, the source wavelet can be specified by an amplitude spectrum and a phase spectrum in the frequency domain:

$$
s(t) \underset{F T}{\Rightarrow} S(\omega)=|s(\omega)|\{\exp j \phi(\omega)\} \text {. }
$$

In general, reflection and attenuation coefficients are frequency-dependent. The properties in the frequency domain can be specified by Fourier transformation of the time domain filters $r_{m}(t)$ and $a_{m}(t)$ :

$$
\begin{aligned}
& r_{m}(t) \underset{\mathrm{FT}}{\Rightarrow} \mathrm{R}_{\mathrm{m}}(\omega)=\left|\mathrm{R}_{\mathrm{m}}(\omega)\right| \exp \left\{j \psi_{\mathrm{R}}(\omega)\right\}, \\
& \mathrm{a}_{\mathrm{m}}(\mathrm{t}) \underset{\mathrm{FT}}{\mathrm{FT}} \mathrm{A}_{\mathrm{m}}(\omega)=\left|\mathrm{A}_{\mathrm{m}}(\omega)\right| \exp \left\{j \psi_{\mathrm{A}}(\omega)\right\} .
\end{aligned}
$$

Hence, assigning depth coordinates $z_{0}$ to the acquisition plane and $z_{m}$ to the $\mathrm{m}^{\text {th }}$ boundary, the one-dimensional model given in Eq. (V-1) can be written, after Fourier transformation to the frequency domain and under assumption of a noise-free situation:

$$
\begin{aligned}
P\left(z_{o}, \omega\right) & =\sum_{m} A^{\prime}\left(\Delta z_{m}, \omega\right) R\left(z_{m}, \omega\right) \exp \left\{-j \omega \tau_{m}\right\} \\
& =S(\omega) \sum_{m} A\left(\Delta z_{m}, \omega\right) R\left(z_{m}, \omega\right) \exp \left\{-j \omega \tau_{m}\right\} \cdot
\end{aligned}
$$


Here, the subscripts $m$ for the functions $R_{m}$ and $A_{m}$, $A_{m}^{\prime}$ have been replaced by variables $z_{m}$ and $\Delta z_{m}=\left|z_{m}-z_{0}\right|$ respectively, in order to indicate explicitly that the former function applies to a depth level, the latter to a depth interval.

If the medium and/or the configuration of sources and detectors is two- or three-dimensional, the wave propagation between surface and boundaries can no longer be described by one-dimensional functions A as above. Instead, propagation through a layer is now described by a two- or three-dimensional spatial wavelet $\mathrm{W}$, which in general is a complex function in the space-frequency domain:

$$
\mathrm{W}=\mathrm{W}(\mathrm{x}, \mathrm{y}, \Delta \mathrm{z}, \mathrm{w}) \quad(\text { three-dimensional }) \quad(\mathrm{V}-5 \mathrm{a})
$$

or

$$
\mathrm{W}=(\mathrm{x}, \Delta \mathrm{z}, \mathrm{w}) \quad(\text { two-dimensional })
$$

In general, $W$ is treated as a function of lateral coordinate(s) $x$ (and $y$ ) with depth interval $\Delta z$ and frequency component $\omega_{i}$ as parameters. Hence, confining our discussion to the two-dimensional situation we write:

$$
\begin{aligned}
W & =W\left(x, \Delta z, \omega_{i}\right) \\
& =\operatorname{Re}\{W(x)\}+\left.j \operatorname{Im}\{W(x)\}\right|_{\Delta z, \omega_{i}} \\
& =\left.|W(x)| \exp \{j \phi(x)\}\right|_{\Delta z, \omega_{i}} .
\end{aligned}
$$

After Fourier transformation to the wavenumber-frequency domain, the two-dimensional spatial wavelet can be specified by a spatial amplitude spectrum and a spatial phase spectrum:

$$
W\left(x, z_{z}, \omega_{i}\right) \underset{F T}{\Rightarrow} \tilde{W}\left(k_{x}, \Delta z, \omega_{i}\right)=\left.\left|\widetilde{W}\left(k_{x}\right)\right| \exp \left\{j \Phi\left(k_{x}\right)\right\}\right|_{\Delta z, \omega_{i}} \cdot \quad(V-7)
$$

In subsection II.3.1 we derived that, using the spatial wavelet concept, a t w o -dimensional multi-trace zero-offset pressure recording in acquisition plane $z=z_{0}$ can be formulated in the space-frequency domain as follows:

$$
\begin{aligned}
P_{Z 0}\left(x, z_{0}, \omega_{i}\right) & =\sum_{m} W^{\prime}\left(x, \Delta_{m}, \omega_{i}\right) * R_{Z 0}\left(x, z_{m}, \omega_{i}\right)+N\left(x, z_{0}, \omega_{i}\right) \\
& =S\left(\omega_{i}\right) \sum_{m} W_{Z 0}\left(x, \Delta z_{m}, \omega_{i}\right) * R_{Z 0}\left(x, z_{m}, \omega_{i}\right)+N\left(x, z_{0}, \omega_{i}\right),
\end{aligned}
$$


where:

$S\left(\omega_{i}\right) \quad$ is the spectral component of the source wavelet for frequency $\omega_{i}$, as given by Eq. (V-2),

$\mathrm{W}_{\mathrm{ZO}}\left(\mathrm{x}, \Delta_{\mathrm{m}}, \mathrm{w}_{\mathrm{i}}\right)$ is the band-1imited spatial wavelet $\left(\left|\mathrm{k}_{\mathrm{x}}\right| \leqq \mathrm{k}_{\mathrm{x} \text {, max }}\right)$ determining, for zero-offset acquisition, the two-way wave propagation between acquisition $p l a n e ~ z=z_{0}$ and depth plane $z=z_{m}$, for frequency $\omega_{i}$, with $\Delta z_{m}=\left|z_{m}-z_{0}\right|$,

$\mathrm{R}_{\mathrm{ZO}}\left(\mathrm{x}, \mathrm{z}_{\mathrm{m}}, w_{\mathrm{i}}\right)$ is the zero-offset reflectivity distribution in plane $z=z_{m}$, for frequency $\omega_{i}$,

$\mathrm{N}\left(\mathrm{x}, \mathrm{z}_{0}, w_{i}\right)$ is the additive noise distribution in the acquisition plane, with frequency $\omega_{i}$.

Now, two remarks of fundamental importance should be made:

(1) The one-dimensional model formulated in Eqs. $(V-1)-(V-4)$ is fully consistent with the two-dimensional model given in Eq. (V-8), since Eq. (V-4) can be written in the convolution representation of Eq. (V-8) defining:

$$
\mathrm{W}_{Z 0}\left(\mathrm{x}, \Delta_{\mathrm{m}}, \omega_{i}\right)=\delta(\mathrm{x}) \mathrm{A}\left(\Delta_{\mathrm{m}}, \omega_{i}\right) \exp \left\{-j \omega_{i} \tau_{m}\right\}
$$

(2) There is a strong formal analogy between Eqs. $(V-1)$ and $(V-8)$, which is specified in table $\mathrm{V} / 1$.

\section{Table V/1}

Analogous elements in Eqs. $(\mathrm{V}-1)$ and $(\mathrm{V}-8)$.

\begin{tabular}{|l||l|}
\hline time domain, Eq. $(V-1)$ & space-f requency domain, Eq. $(V-8)$ \\
\hline$p(t)$ & $P_{Z O}\left(x, z_{o}, \omega_{i}\right)$ \\
$a_{m}^{\prime}\left(t-\tau_{m}\right)=s(t) * a_{m}\left(t-\tau_{m}\right)$ & $W^{\prime}\left(x, \Delta_{m}, \omega_{i}\right)=S\left(\omega_{i}\right) W_{Z 0}\left(x, \Delta_{m}, \omega_{i}\right)$ \\
$r_{m}(t)$ & $R_{Z O}\left(x, z_{m}, \omega_{i}\right)$ \\
$n(t)$ & $N\left(x, z_{o}, \omega_{i}\right)$ \\
\hline
\end{tabular}

The analogy is also seen in the interpretation of the two equations. Eq. (V-1) means:

In modeling temporal response $p(t)$, a sequence of reflectivities $r_{m}(t)$ is convolved with temporal wavelets

$$
a_{m}^{\prime}\left(t-\tau_{m}\right)=s(t) * a_{m}\left(t-\tau_{m}\right)
$$


After Fourier transformation, these temporal wavelets can be specified by temporal amplitude and phase spectra according to Eqs. $(\mathrm{V}-2,3)$.

Eq. (V-8) means:

In modeling zero-offset spatial response $\mathrm{P}_{\mathrm{ZO}}\left(\mathrm{x}, \mathrm{z}_{0}, \omega_{i}\right)$, where $\omega_{i}$ is a parameter, a zero-offset reflectivity distribution $\mathrm{R}_{\mathrm{ZO}}\left(\mathrm{x}, \mathrm{z}_{\mathrm{m}}, \omega_{i}\right)$ is convolved with a complex spatial wavelet $W^{\prime}\left(x, \Delta_{z}, w_{i}\right)=S\left(w_{i}\right) W_{Z 0}\left(x, \Delta_{m}, \omega_{i}\right), m=1,2, \ldots, M$

for each depth level $\mathrm{z}_{\mathrm{m}}$. After Fourier transformation, these spatial wavelets can be specified by spatial amplitude and phase spectra according to Eq. (V-7).

From the formal analogy between data in the time domain and in the spacefrequency domain, we may draw an important conclusion:

A11 considerations, dedicated in chapter IV to ME-norms applied to data in the space-time $(x, t)$ domain (i.e. functions of $t i m e$ for a number of $s p a c e$ coordinates), are also valid for ME-norms applied to zero-offset data in the space-frequency $(x, \omega)$ domain (i.e. functions of $s$ p a $c$ e coordinate for a number of $f \mathrm{r}$ e $q \mathrm{u}$ e $\mathrm{n}$ c $\mathrm{y}$ values).

Examples of such considerations:

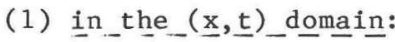

The value of an ME-norm applied to a temporal response (at $x_{=} x_{0}$ ) is a measure of the vertical resolving power of the response, giving insight how well different events can be detected separately in the $t$ - and $z$-directions. similarly, in the $(x, \omega)$ domain:

The value of an ME-norm applied to a spatial response (for $\omega=\omega_{i}$ ) is a measure of the lateral resolving power of that response, giving insight how well different events can be detected separately in the $\mathrm{x}$-direction.

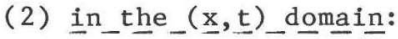

The value of an ME-norm applied to a temporal response (at $x_{=} x_{0}$ ) is determined by the compactness of the temporal wavelets together with the sparsity of the (vertical) reflector distribution. 
similiarly, in the $(x, \omega)$ domain:

The value of an ME-norm applied to a spatial response ( for $\omega=\omega_{i}$ ) is determined by the compactness of the spatial wavelets together with the sparsity of the reflectivity distribution which is now extended in two dimensions ( $x$ and $z$ ).

Or, in particular, for one boundary at $z=z_{1}$ :

The value of an ME-norm applied to a spatial response (for $\omega=\omega_{i}$ ) is determined by the compactness of the spatial wavelet together with the sparsity of the zero-offset reflectivity distribution at depth $z_{1}$.

(3) in_the_( $\underline{x}, \underline{t})$ _domain:

The compactness of the temporal wavelet, being an inverse measure of dispersion, is determined by temporal bandwidth and temporal phase spectrum. similarily, in the $(x, \omega)$ domain:

The compactness of the spatial wavelet, being an inverse measure of spatial dispersion, is determined by spatial bandwidth and spatial phase spectrum.

(4) in_the_( $\underline{x}, \underline{t})$ _domain:

For a multi-trace data set (i.e. temporal responses at $x_{1} x_{1}, x_{2}, \ldots, x_{N}$ ), multi-trace ME-norms can be defined, weighting the contributions of each individual trace with a suitable trace weighting factor. similararly, in the $(x, \omega)$ domain:

Defining a set of spatial responses for frequency sequence $\omega=\omega_{1}, \omega_{2}, \ldots, \omega_{M}$ a multi-channel data set, multi-channel ME-norms can be defined, weighting the contributions of each individual channel with a suitable channel weighting factor. Intuitively, it seems appropriate to relate these weighting factors, in some way, to the ratio of the amplitude spectrum $|S(\omega)|$ of the temporal wavelet and the noise spectrum $|N(\omega)|$.

Besides all similarities mentioned above, there is a basic difference between data in the $(x, t)$ and the $(x, \omega)$ domain: data in the $(x, t)$ domain are real, whereas data in the $(x, \omega)$ domain generally are complex. ME-norms were applied to temporal (rea1) data after transformation to some positive definite amplitude parameter, see section IV.2. In order to maintain the full analogy, we shall apply ME-norms to spatial (complex) data after transformation to some real positive definite amplitude parameter, for which the mo d u 1 u $s$ (or its powers) is suitable. Hence, discussing dispersion of a spatial wavelet, sparsity of a spatial reflectivity distribution, resolving power of a spatial 
response, etc., we in fact relate these properties to the moduli of these spatial functions.

To illustrate the above arguments, we sha11 now apply ME-norms to the spatial wavelet $\mathrm{W}_{\mathrm{ZO}}$ appearing in Eq. $(\mathrm{V}-8)$. In subsection II.3.1 we showed that a good approximation of this band-1imited spatial wavelet, describing the two-way wave propagation between surface $z=z_{0}$ and a depth plane at $z=z_{m}$ in a twodimensional zero-offset pressure recording, is given by the two-dimensional farfield version of the homogeneous extrapolation operator with 'half-velocity' (or double frequency) substitution:

$$
\begin{aligned}
W_{Z O}\left(x, \Delta z_{m}, w_{i}\right) & \approx W\left(x, \Delta z_{m}, 2 \omega_{i}\right) \\
& =\Delta z_{m} \sqrt{\frac{j k}{\pi}} \frac{\exp (-2 j k r)}{r^{3 / 2}},
\end{aligned}
$$

with

$$
\Delta z=\left|z_{0}-z_{m}\right|
$$

and

$$
r^{2}=(\Delta x)^{2}+\left(\Delta z_{m}\right)^{2}
$$

see Fig. V-1.

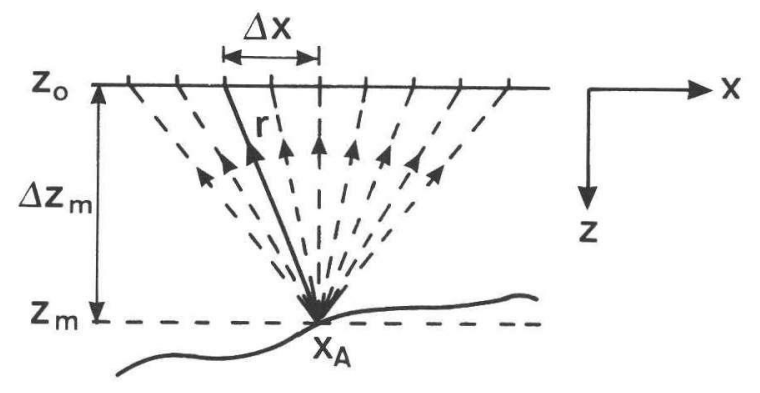

Figure V-1: Geometry to Eq. $(\mathrm{V}-10)$.

Note that $2 \mathrm{k}\left(=\omega_{i} /(\mathrm{c} / 2)\right)$ has replaced $\mathrm{k}$ in the original operator as specified by Eq. $(I I-5)$.

It should be recalled here that, in this two-dimensional zero-offset mode1, any boundary is represented by a configuration of secondary line sources with 

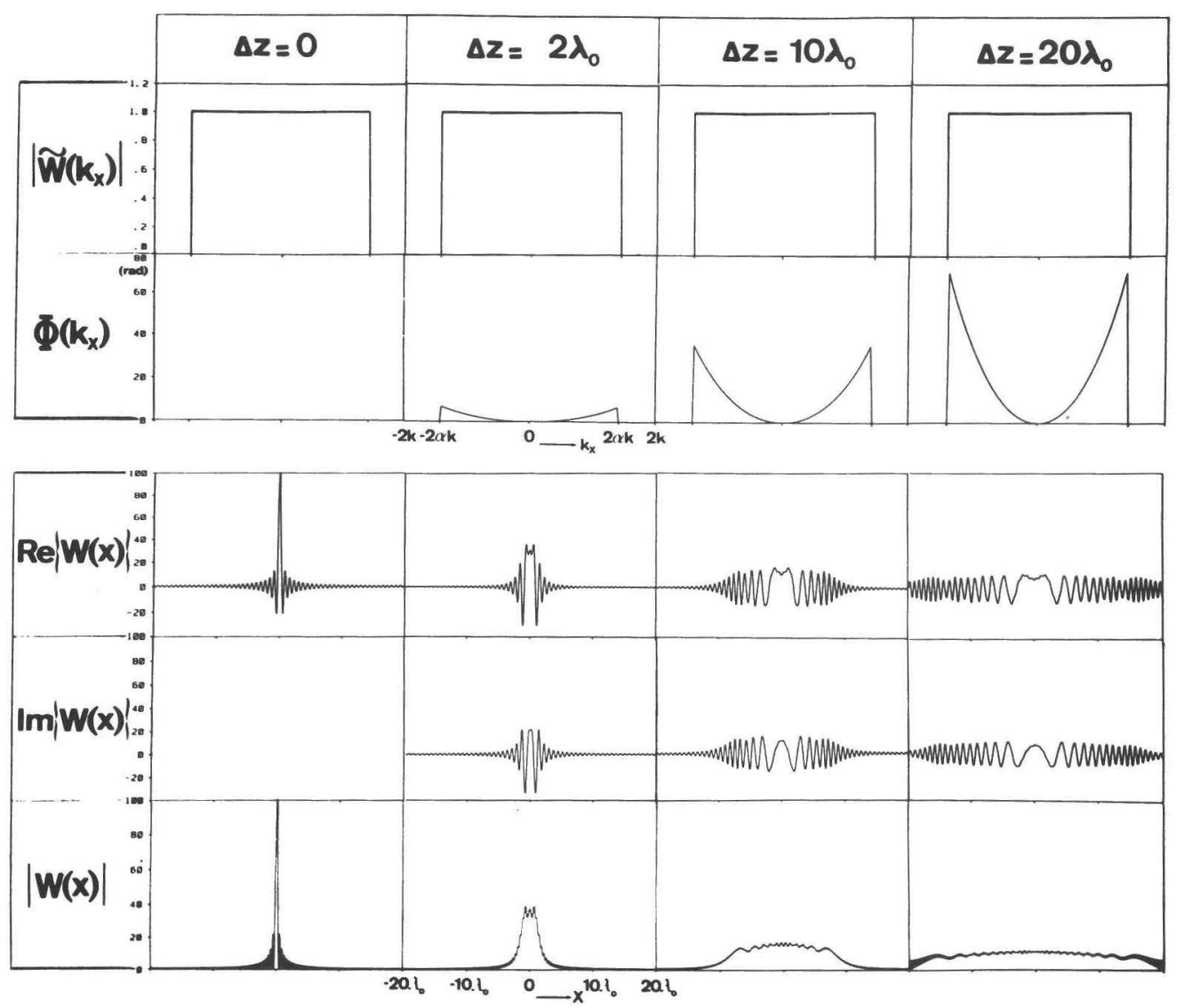

Figure V-2: Band-limited spatial wavelets describing zero-offset wave propagation.

bandwidth: $\left|k_{x}\right| \leqq 2 \alpha_{k}, \alpha=\frac{1}{2} \sqrt{2}$

propagation intervals: $\Delta_{z}=0,2 \lambda_{0}, 10 \lambda_{0}, 20 \lambda_{0} ; \lambda_{0}=2 \pi / k$

From top to bottom, amplitude spectra, phase spectra, real parts, imaginary parts and modulus (i.e. directivity) patterns of spatial wavelets are displayed. 
dipole character. The strength of each dipole is given by the local reflectivity. Be aware that this a $m$ a $t h$ e $m$ a $i c$ a 1 representation and not a physical description of the boundary.

We saw (subsection II.3.1) that the spatial Fourier transform of Eq. (V-10) is given by:

$$
\widetilde{W}\left(k_{x}, \Delta z, 2 \omega_{i}\right)=\exp \left(-j k_{z} \Delta z\right)
$$

with

$$
\mathrm{k}_{z}^{2}=4 \mathrm{k}^{2}-\mathrm{k}_{\mathrm{x}}^{2}
$$

This means that the spatial phase angle $\left|\mathrm{k}_{\mathrm{z}} \Delta_{\mathrm{z}}\right|$ increases with propagation interval $\Delta \mathrm{z}$ for a given $\mathrm{k}_{\mathrm{x}}$-value.

This is illustrated in Fig. V-2 where, for a temporal frequency $\omega_{0}$, the above spatial wavelet is considered within a limited spatial bandwidth given by (see Fig. V-2a)

$$
\begin{aligned}
& \left|\widetilde{W}\left(k_{x}\right)\right|=1,\left|k_{x}\right| \leqq 2 \alpha k \\
& \left|\widetilde{W}\left(k_{x}\right)\right|=0,\left|k_{x}\right|>2 \alpha k,
\end{aligned}
$$

where $\mathrm{k}=\omega_{\mathrm{o}} / \mathrm{c}$ and $\alpha$ is a parameter here chosen $\frac{1}{2} \sqrt{2}$. For the propagation interval $\Delta \mathrm{z}$ the values $0,2 \lambda_{0}, 10 \lambda_{\mathrm{o}}$ and $20 \lambda_{\mathrm{o}}$ have been chosen, where

$$
\lambda_{0}=\frac{2 \pi c}{\omega_{0}}=\frac{2 \pi}{k} .
$$

Besides the spatial amplitude and phase spectra according to Eq. (V-11), Fig. V-2 shows, as a function of $x$, the real parts, the imaginary parts and the modulus patterns (directivity patterns) of the corresponding spatial wavelets.

It is seen that, as expected, $i \mathrm{n}$ creasing propagation interval $\Delta \mathbf{z}$ leads to an i n creasing spatial phase spectrum and to $i$ n creasing spatial dispersion of the wavelet in the $\mathrm{x}$-domain. Minimum dispersion is found for the zero-phase wavelet. Moreover, it is seen that for non-zero-phase wavelets the real and imaginary parts have a sweep character, which corresponds with what we found for time wavelets, see Fig. IV-6. 
ME-norms of the form

$$
v=\overline{q_{i} F\left(q_{i}\right)}
$$

are applied to the modulus patterns of Fig. V-2. Note that the data are already presented in the form of a positive definite amplitude parameter, so that a transformation as suggested in subsection IV.3.1 is not necessary and hence

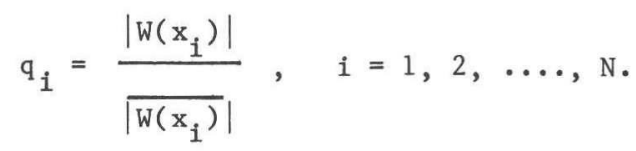

Using entropy functions $F(q)=q^{\frac{1}{2}}, q$ and $q^{2}$ respectively, we find the values of $\mathrm{V}$ given in table $\mathrm{V} / 2$. For each entropy function, the value of $\mathrm{V}$ for $\Delta z=0$ is normalized to 1.00 .

\section{Table V/2}

Normalized values of ME-norms $V$ for the modulus patterns of the spatial wavelets specified in Fig. $v-2$.

\begin{tabular}{|l|l|l|l|}
\cline { 2 - 4 } \multicolumn{1}{c|}{} & \multicolumn{3}{|c|}{$V=\frac{1}{N} \sum_{i} q_{i} F\left(q_{i}\right)$} \\
\hline$\Delta z$ & $F(q)=q^{\frac{1}{2}}$ & $F(q)=q$ & $F(q)=q^{2}$ \\
\hline \hline 0 & 1.00 & 1.00 & 1.00 \\
\hline $2 \lambda_{0}$ & 0.72 & 0.40 & 0.10 \\
\hline $10 \lambda_{0}$ & 0.43 & 0.13 & 0.01 \\
\hline $20 \lambda_{0}$ & 0.35 & 0.08 & $0.00(3)$ \\
\hline
\end{tabular}

It is seen that, for all entropy functions, the values of $\mathrm{V}$ decrease with increasing $\Delta z$, i.e. with increasing wavelet dispersion, as expected. The decrease is strongest for high-power entropy functions.

For a given choice of spatial bandwidth parameter $\alpha$ - see Eq. (V-12) - the spatial bandwidth increases linearly with temporal frequency, as was illustrated in the $\left(k_{x}, k\right)$ diagram of Fig. III-la. 


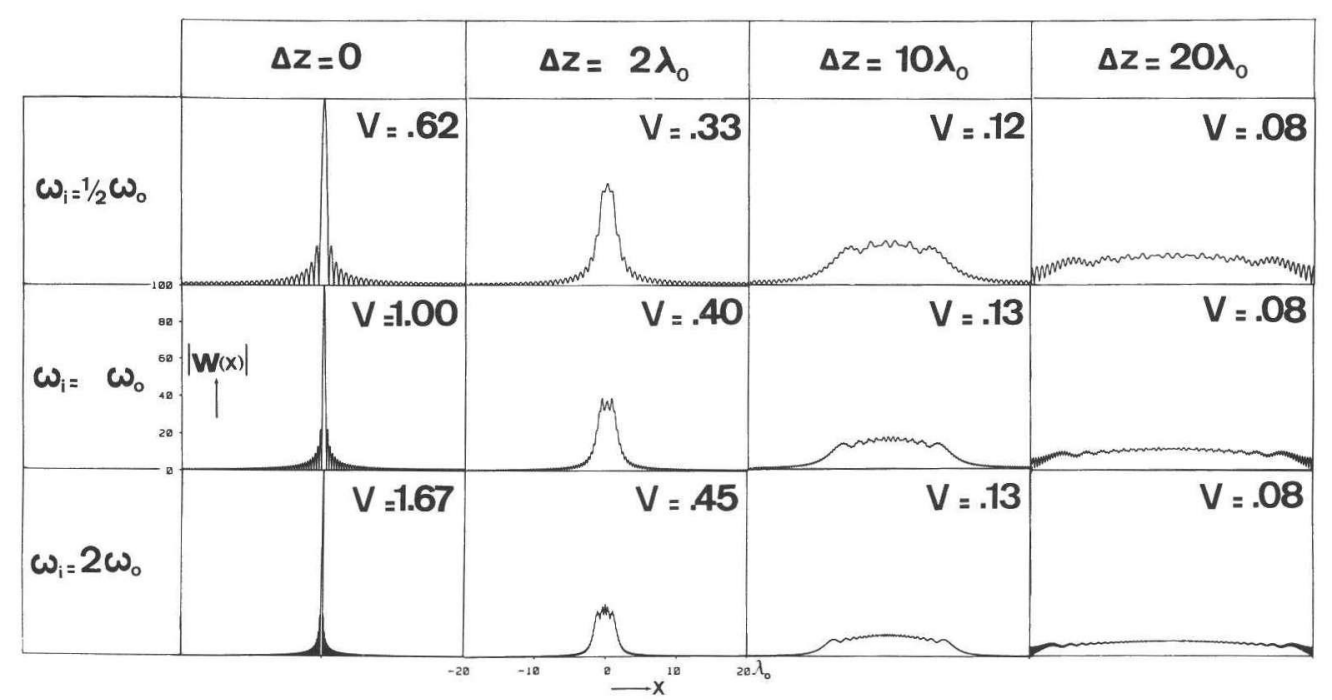

Figure V-3: Influence of temporal frequency on the directivity patterns of spatial wavelets as specified in Fig. V-2. From top to bottom, directivity patterns are displayed for $\omega_{i}=\frac{1}{2} \omega_{0}$, $\omega_{0}$ and $2 \omega_{0}$ respectively, $\omega_{0}$ being the frequency considered in Fig. V-2. The modulus value for $x=0$ is normalized to 100 for each f requency .

ME-norm values, applying amplitude parameter $a=|W|$ and entropy function $F(q)=q$, are given in the figures, normalized to the value for $\omega_{i}=\omega_{0}, \Delta z=0$. 

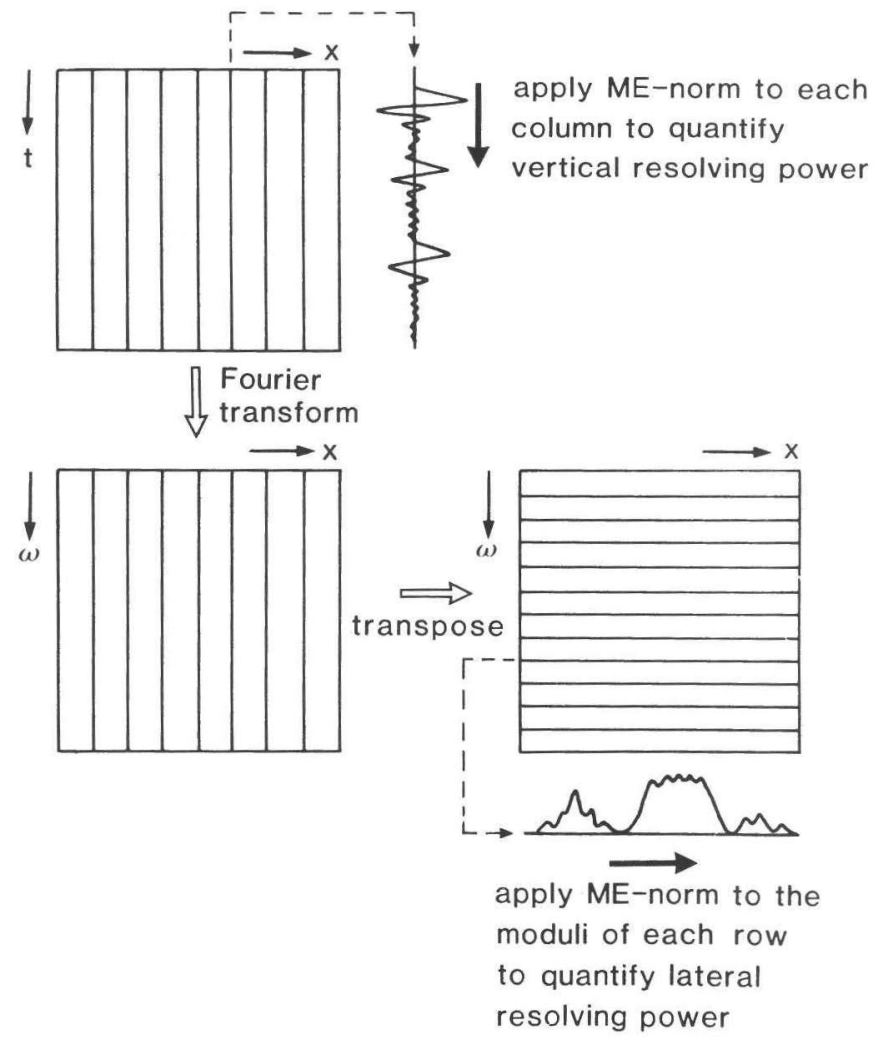

Figure V-4: Schematic application of ME-norms to a two-dimensional data set. 
In Figs. V-3 the directivity patterns of spatial wavelets are shown for the same value of $\alpha$ and the same depth intervals $\Delta z$ as in Fig. $V-2$, but now for temporal frequencies $\frac{1}{2} \omega_{0}, \omega_{0}$ and $2 \omega_{0}$ respectively. The modulus value for $x=0$ is normalized to 100 for each frequency. It is seen that dispersion decreases (i.e. compactness increases) with increasing temporal frequency and corresponding spatial bandwidth. In the figures, the values of ME-norm $V$ are given, for the amplitude parameter of Eq. $(V-15)$ and for entropy function $F(q)=q$, normalized to the value for $\omega_{i}=\omega_{0}, \Delta z=0$. As expected, $V$ increases with increasing frequency, but less strongly for increasing $\Delta z$.

Above, we applied ME-norms to single spatial wavelets. To complete this section, we shall now apply ME-norms to simulated two-dimensional zero-offset data sets. Generally, such data sets contain information about the geometry of the underlying two-dimensional medium, especially when the recorded data is processed with some inversion operator in order to eliminate propagation effects, as discussed in chapter II. The information about the medium can be qualified by resolving power of the data in vertical as well as in lateral direction. As we saw earlier in this section, the resolving power in $v e r-$ $t i c$ a 1 direction can be specified by applying an ME-norm in the $s$ p $c e-$ $t i \mathrm{~m} e$ domain along the time axis. The value of this norm which we denote as $V_{x}(t)$ is determined by temporal wavelet dispersion and vertical reflectivity sparsity. The 1 a $t$ e $r$ a 1 resolving power can be specified by applying an ME-norm in the $s \mathrm{p}$ a $\mathrm{c}$ e $-\mathrm{f} \mathrm{r}$ e $\mathrm{q} u$ e $\mathrm{n} \mathrm{y}$ domain along the space axis. The value of this norm which we denote as $v_{\omega}(x)$ is determined by spatial wavelet dispersion and the sparsity of the zero-offset reflectivity distributions in the sub-surface planes. Hence, ME-norms $V_{x}(t)$ and $V_{\omega}(x)$ can be usefu11y applied according to the scheme given in Fig. V-4.

In this way, we first consider the influence of $b$ a $n d w i d t$ on the values of $V_{x}(t)$ and $V_{\omega}(x)$ for the zero-offset image of a regular two-dimensional diffractor distribution embedded in a homogeneous medium. See Fig. V-5, where four image patterns are considered, in which two temporal and two spatial bandwidths are combined. Both temporal and spatial wavelets are zero-phase. Under the figures the values of $V_{x}(t)$ and $V_{\omega}(x)$ are given, normalized to the values for full temporal and full spatial bandwidth (Fig. V-5a). As expected, $V_{x}(t)$ is strongly diminished by temporal bandwidth limitation and not significantly influenced by variation of spatial bandwidth. For $V_{\omega}(x)$ the opposite holds: its value is independent of temporal bandwidth and decreases with decreasing spatial bandwidth. 


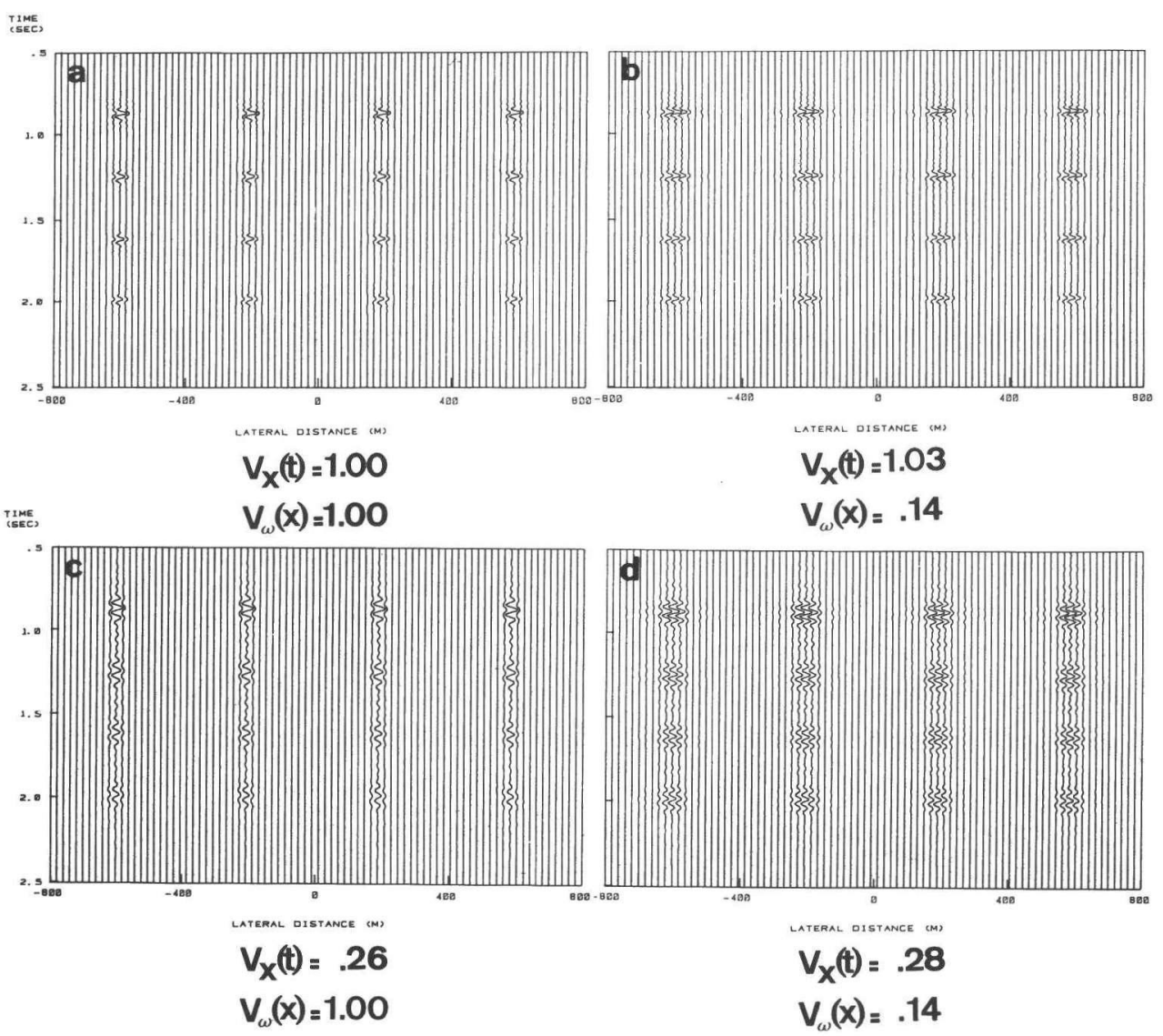

Figure V-5: Image patterns and corresponding ME-norm values of a two-dimensional diffractor grid as a function of temporal and spatial bandwidth.

a: image with full temporal and full spatial bandwidth

b: image with full temporal and limited spatial bandwidth

c: image with limited temporal and full spatial bandwidth

d: image with limited temporal and limited spatial bandwidth.

amplitude parameter: $a=|y|$

entropy function : $F(q)=q^{2}$.

Fig. V-6 concerns the influence on $v_{x}(t)$ and $v_{\omega}(x)$ of $s$ a $r s$ i $y$ variation of a diffractor grid, in vertical as well as in 1ateral direction. The images of the configurations are simulated for given zero-phase temporal and spatial wavelets. Here, $v_{x}(t)$ and $v_{\omega}(x)$ are normalized to the values for reference 
(high) sparsity in both directions (Fig. V-6a). As expected, $V_{x}(t)$ decreases with decreasing vertical sparsity (increasing vertical density) being insensitive to lateral sparsity variations. Contrarily, $v_{\omega}(x)$ is insensitive to vertical sparsity variation and decreases with decreasing lateral sparsity (increasing latera1 density).
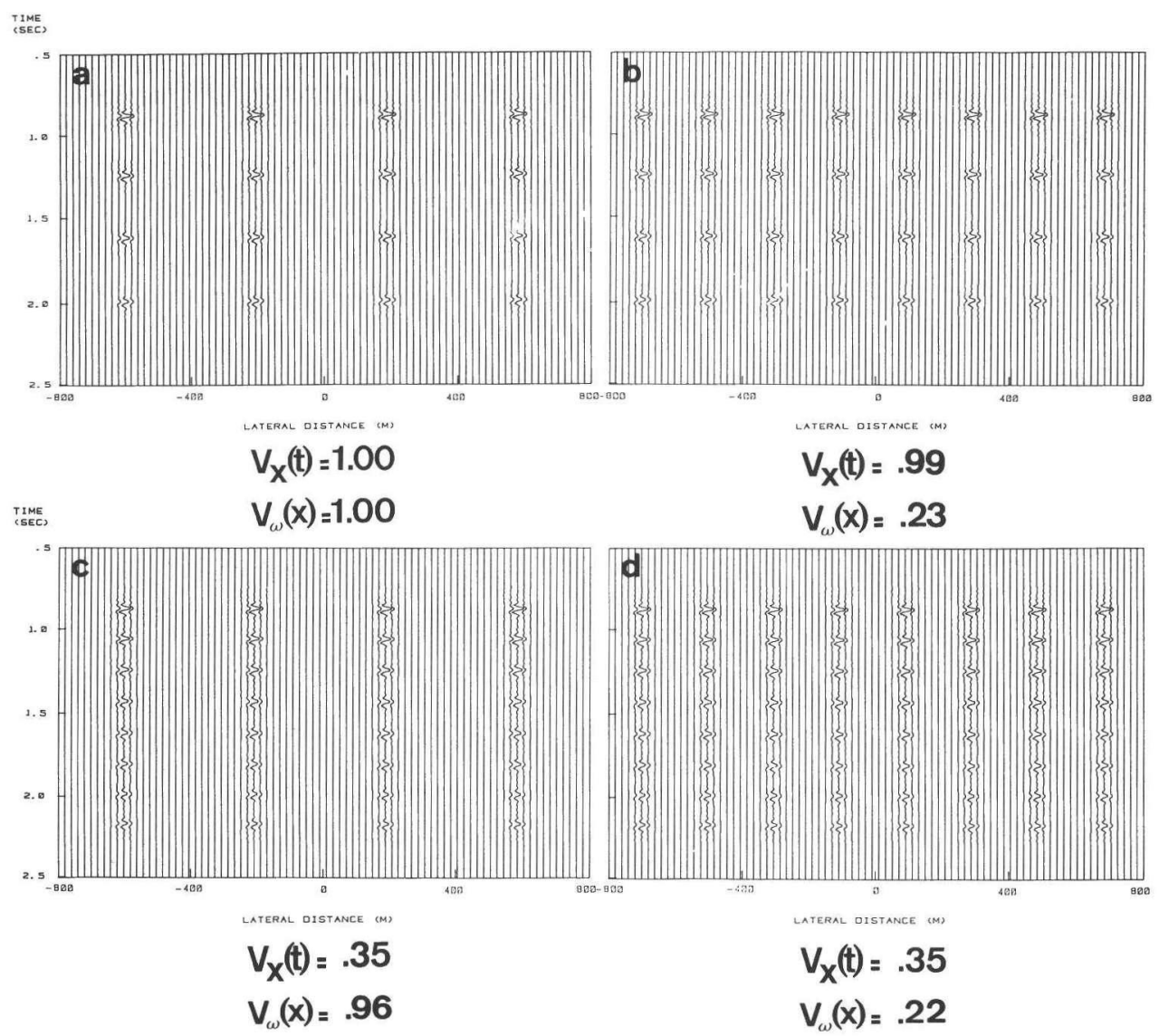

$V_{\omega}(x)=.22$

Figure V-6: Image patterns and corresponding ME-norm values of a two-dimensional diffractor grid as a function of vertical and lateral sparsity.

a: image with reference (high) vertical and lateral sparsities

$\mathrm{b}$ : image with half lateral sparsity (double lateral density)

c: image with half vertical sparsity (double vertical density)

$\mathrm{d}$ : image with half lateral and vertical sparsities

amplitude parameter: $a=|y|$

entropy function : $F(q)=q^{2}$ 


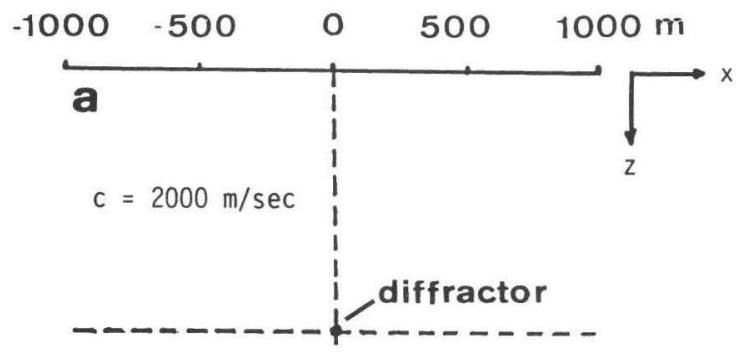

CORRECT VELOCITY

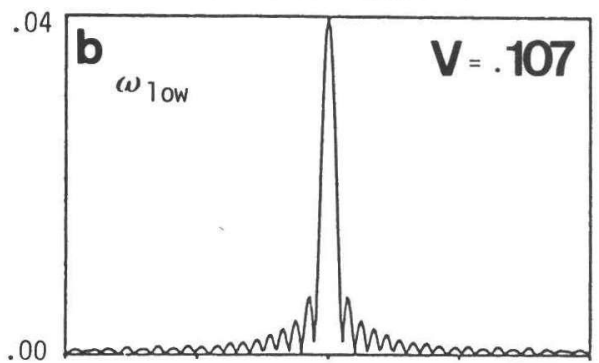

1.00
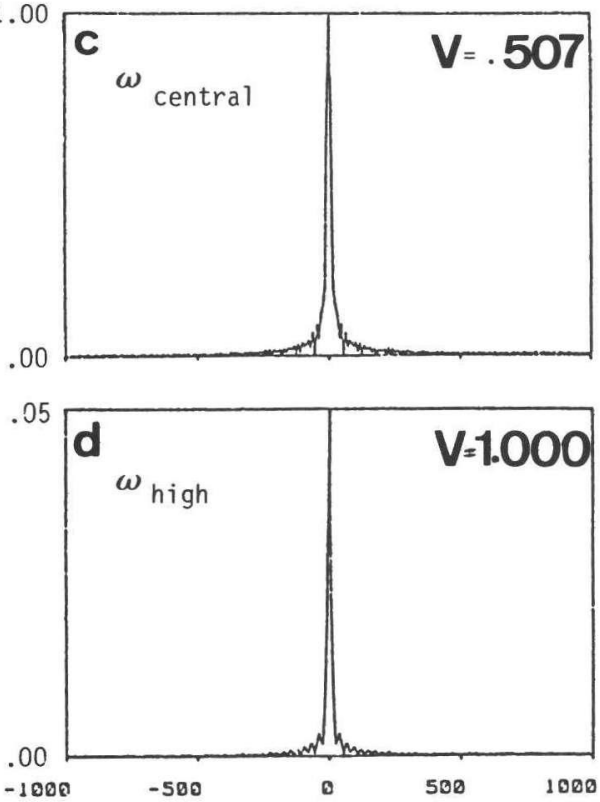

VELOCITY ERROR $10 \%$
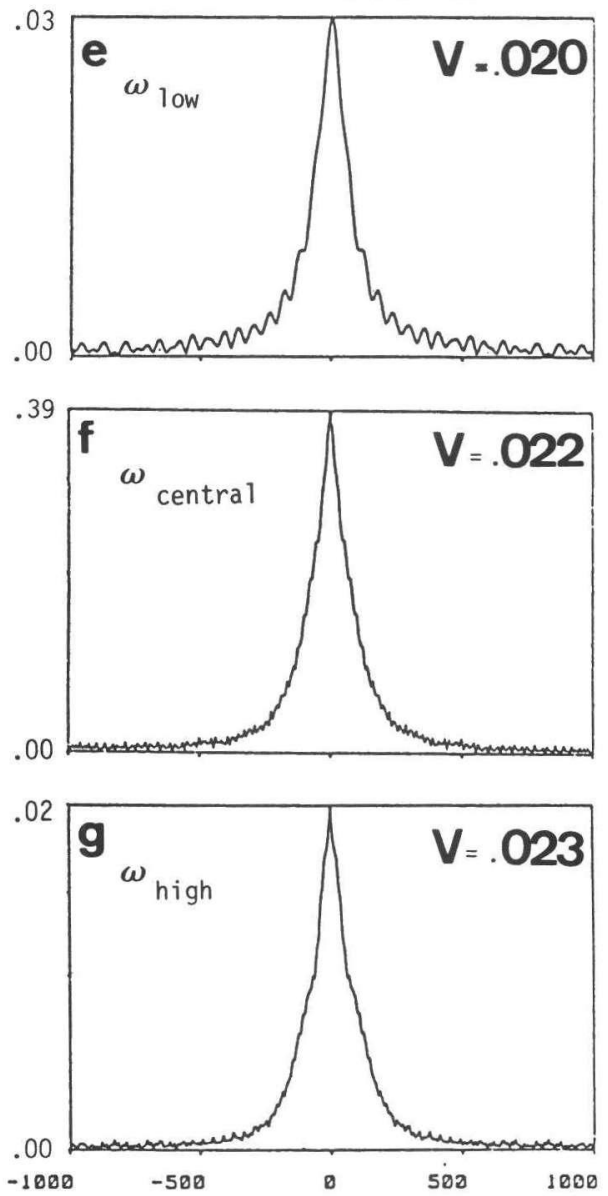

Figure V-7: Modulus patterns of $\mathrm{U}_{\mathrm{ZO}}(\mathrm{x})$, i.e. directivity patterns of an inverted diffractor response, for different frequency components, without $(b, c, d)$ and with $(e, f, g)$ velocity error. The values of ME-norm $\mathrm{V}$ are normalized to the maximum value occurring (d). amplitude parameter: $\mathrm{a}=\left|\mathrm{U}_{\mathrm{ZO}}\right|$ entropy function : $F(q)=q^{2}$ 


\section{V.3 MINIMUM ENTROPY VELOCITY ANALYSIS}

\section{V.3.1 Velocity analysis using minimum entropy norms in the space-frequency}

\section{domain}

In section III.2 we considered band-1imited spatial wavelet $\mathrm{U}_{\mathrm{ZO}}\left(\mathrm{x}, \Delta_{\mathrm{z}}, \omega_{i}\right)$ defined as:

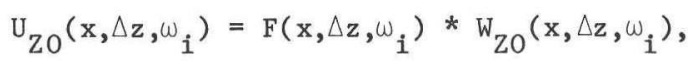

representing zero-offset data from a reflectivity distribution at depth $\Delta \mathrm{z}$ below the acquisition plane after inversion with matched filtering operator $F$. It was shown that the lateral dispersion of this spatial wavelet increases if an erroneous velocity value is inserted into operator F, see Fig. III-2. Since, according to what we concluded in the previous section, $i$ creasing dispersion of a spatial wavelet corresponds with d e creasing ME-norm values, we may expect that the maximum value of an ME-norm applied to spatial wavelet $\mathrm{U}_{\mathrm{ZO}}$ will be found if no velocity errors are present.

The above hypothesis is illustrated and verified in Fig. V-7 where, in simulation, the inverted response is considered of an elementary diffractor positioned at a depth level $z=1000 \mathrm{~m}$ below an aperture length of $2000 \mathrm{~m}$, the medium having a propagation velocity of $2000 \mathrm{~m} / \mathrm{s}$, see Fig. V-7a. Note that this inverted response can be represented by spatial wavelet $U_{z 0}\left(x, z, \omega_{i}\right)$. The directivity pattern of the inverted diffractor response is given for three frequency components $\left(\omega_{i}=\omega_{10 w}, \omega_{\text {central }}, \omega_{\text {high }}\right)$ of a cosine-shaped amplitude spectrum, for correct velocity (Figs. V-7b,c,d) and a velocity error of $10 \%$ (Figs. V-7e,f,g ${ }^{1}$ ). The corresponding values of ME-norm V, normalized to the highest value occurring (Fig. V-7d) are given in the figures. As expected, the values of $\mathrm{V}$ are smaller in case of velocity error. Moreover, it is seen that in the error-free situation the value of $V$ increases with increasing frequency, as it should be, whereas this effect is hardly found when a velocity error is present.

1) The response considered is generated in the $(x, t)$ domain. Due to the specific signal processing applied, the side lobes of the directivity patterns for correct velocity - which should be represented by sinc-functions - are 'randomized', so that these patterns are not fully comparable with those in Fig. III-2. 


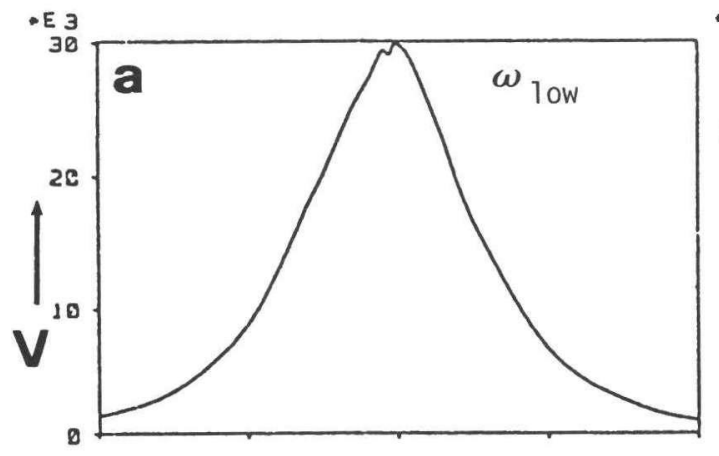

- E 3

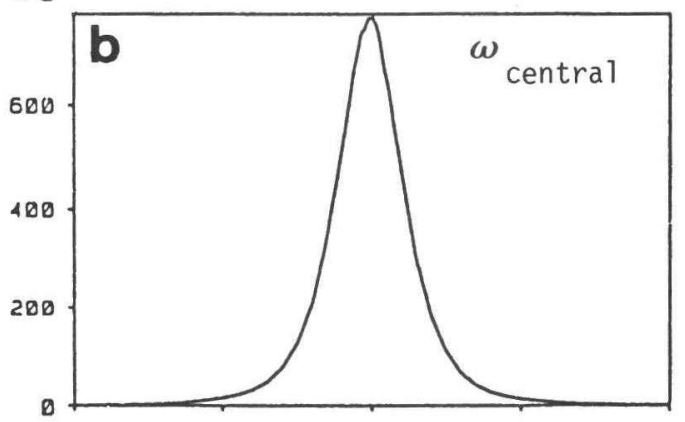

- 6

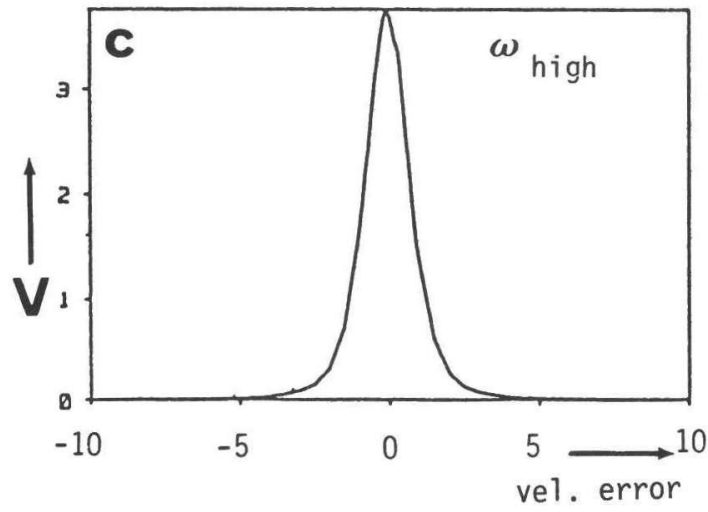

*E 3

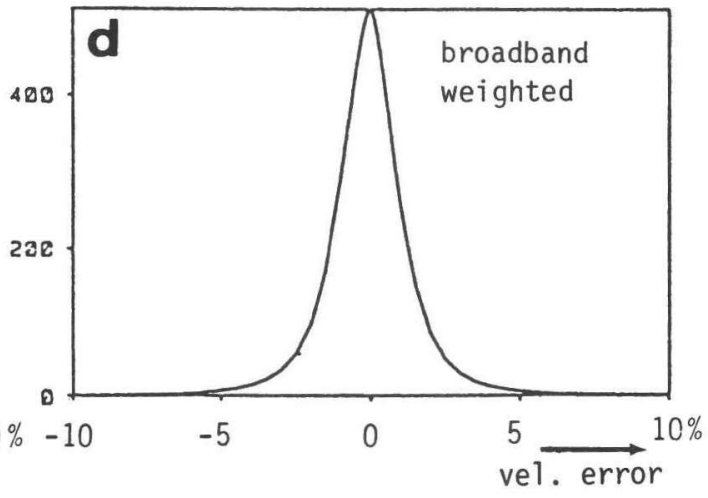

Figure IV-8: ME-norm V as a function of relative velocity error, applied to $\left|\mathrm{U}_{\mathrm{ZO}}(\mathrm{x})\right|$. Results are given for single frequency components $(\mathrm{a}, \mathrm{b}, \mathrm{c})$ and summed over all frequencies after weighting with the temporal amplitude spectrum (d). Noise-free situation. amplitude parameter: $a=\left|\mathrm{U}_{\mathrm{ZO}}\right|$
entropy function : $\mathrm{F}(\mathrm{q})=\mathrm{q}^{4}$ 
For the same configuration, the variation of ME-norm $V$ for a continuous velocity error range is given in Fig. $\mathrm{V}-8$, where the relative velocity error is varied between $-10 \%$ and $+10 \%$. Figs. V-8a,b,c give the V-curves for the spatial wavelets $\mathrm{U}_{\mathrm{ZO}}\left(\mathrm{x}, \mathrm{z}, \omega_{\mathrm{i}}\right)$ corresponding with low, central and high frequency respectively. Fig. V-8d gives $V$ as a multi-channe1 ME-norm, summed over all frequencies weighted with the cosine-shaped amplitude spectrum. Here, and in following velocity detection examples, entropy function $\mathrm{F}(\mathrm{q})$ has been chosen $\mathrm{q}^{4}$, since optimal discrimination between velocity error effects appears to require a high-power entropy function. (See also subsection VI.3.1 of the next chapter.)

In Fig. V-8 we see, as expected:

(1) ME-norm $V$ shows a maximum for $\Delta c=0$, if taken as a single-frequency as well as a multi-channel norm.

(2) For higher frequencies, the V-curves become narrower, which means a more accurate detection of the correct velocity value.

(3) For higher frequencies, ME-norm V obtains a higher maximum value (compare the scales of the vertical axes), so that in the multi-channel norms (Fig. $\mathrm{V}-8 \mathrm{~d})$ the contributions of the higher frequencies dominate, resulting in a relatively narrow V-curve.

In Fig. V-9 the calculations of Fig. V-8 are repeated, but now after addition of $n \circ i s$ e to the simulated recording (signal-to-noise ratio $0 \mathrm{~dB}$ ). It is seen that from frequency components with a low signal-to-noise ratio (Figs. $\mathrm{V}-9 \mathrm{a}, \mathrm{c})$ no velocity information can be obtained, but only from components where this ratio is high (Fig. V-9b). An appropriate spectral weighting function is in this situation:

$$
w(\omega)=\frac{|S(\omega)|^{2}}{|S(\omega)|^{2}+|N(\omega)|^{2}},
$$

$N(\omega)$ being the noise spectrum; see Fig. V-9d.

From the above results, the following general and highly important conclusion can be drawn: Application of minimum entropy norms to inverted zero-offset data in the
space-frequency domain is a tool for velocity analysis. 

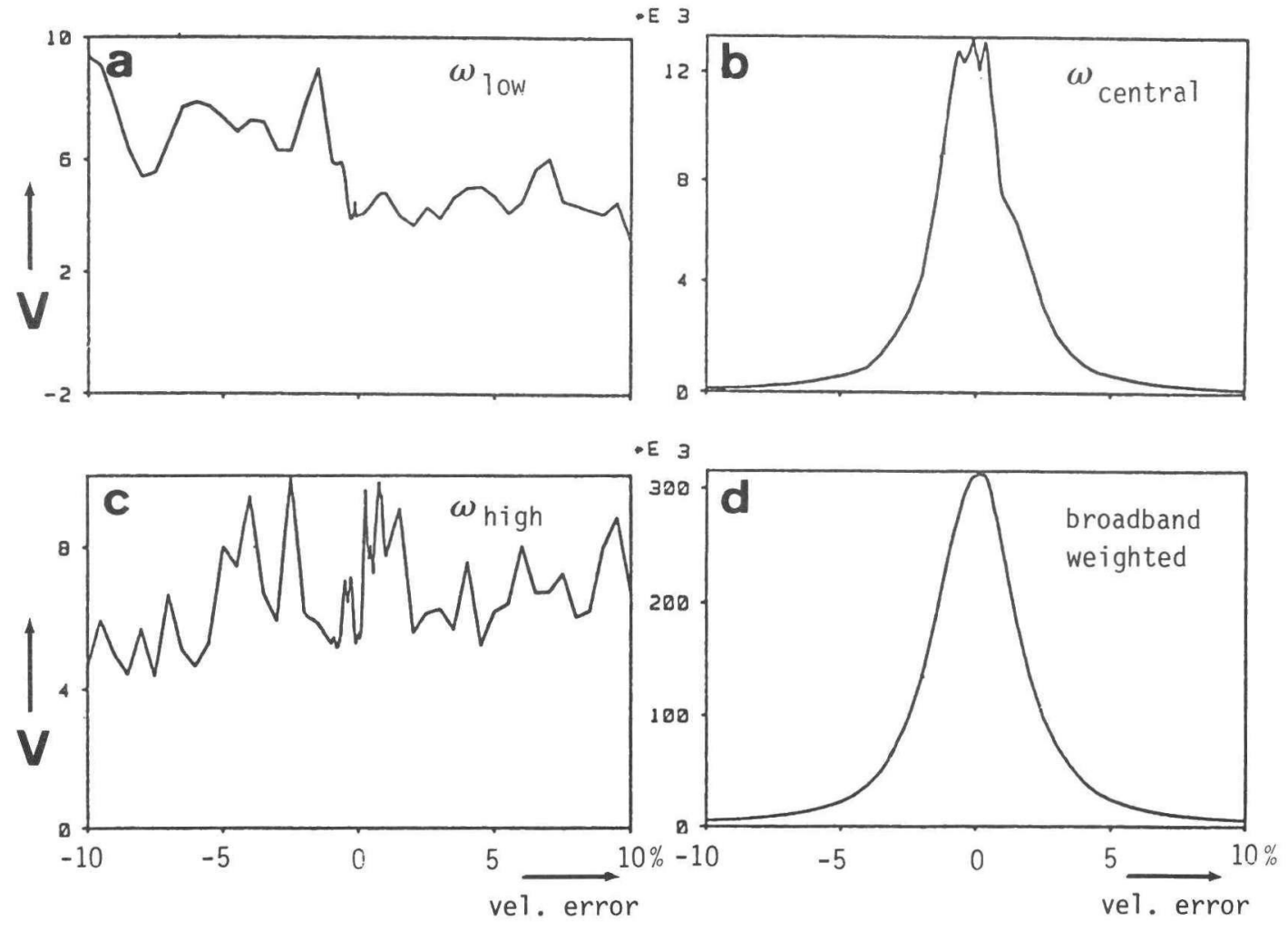

Figure V-9: $M E-n o r m ~ V$ as a function of relative velocity error, applied to $\left|\mathrm{U}_{\mathrm{ZO}}(\mathrm{x})\right|$. Results are given for single frequency components $(a, b, c)$ and summed over all frequencies (d) after weighting with the function of Eq. (V-17).

signal-to-noise ratio: $0 \mathrm{~dB}$

$\begin{array}{ll}\text { amplitude parameter } & : \mathrm{a}=\left|\mathrm{U}_{\mathrm{ZO}}\right| \\ \text { entropy function } & : \mathrm{F}(\mathrm{q})=\mathrm{q}^{4}\end{array}$ 
We shall call this technique $\underline{M} i \mathrm{n} i \mathrm{~m}$ m $\underline{\mathrm{E}} \mathrm{n} \mathrm{r} \circ \mathrm{p}$ y $\underline{\mathrm{V}} \mathrm{e} 1$ o c $\mathrm{i} y$ A $\mathrm{n}$ a 1 y $\mathrm{s} i \mathrm{~s}$, abbreviated as MEVA.

More specifically, we conclude from Figs. V-8,9 that MEVA can in principle be performed by applying an ME-norm to one spectral component of the inverted spatial wavelet. This component should have a high signal-to-noise ratio and, preferably (for detectability purposes), a high frequency. To further enhance the detectability, the contributions of a number of strong spectral components should be added, preferably after weighting with a factor based on the signalto-noise ratio, e.g. as given by $\mathrm{Eq} \cdot(\mathrm{V}-17)$. It should be mentioned that, due to the $\circ \mathrm{r} \mathrm{t}$ h $\mathrm{g} \circ \mathrm{n}$ a $1 \mathrm{i} \mathrm{t}$ of the spectral components, the spatial wavelets are independent of each other and, hence, yield independent contributions to the multi-channel ME-norm. Note that, if the addition step is omitted, MEVA estimates velocity as a function of frequency, which is relevant for media with frequency-dependent properties (e.g. absorption).

To conclude this subsection, we shall make some remarks on the applicability of MEVA. Above, as input data for MEVA was used the band-1imited spatial wavelet $\mathrm{U}_{\mathrm{ZO}}$, representing the inverted zero-offset response of a $\mathrm{s} i \mathrm{n} g \mathrm{e}_{\mathrm{dif}}$ fractor. In practice, zero-offset (or stacked) data contain diffraction energy from a spatial distribution of diffractors. Then, MEVA is applied to inverted zero-offset responses to be written in the form:

$$
\sum_{m} \sum_{i} U_{z 0}\left(x-x_{i}, \Delta z_{m}, \omega_{i}\right) .
$$

For data as such, the value of ME-norm $V$ not only depends on the dispersion (compactness) of spatial wavelets $\mathrm{U}_{\mathrm{ZO}}$, but also on diffractor $\mathrm{s}$ p a $\mathrm{r}$ s $\mathrm{i}$ $t \mathrm{y}$ in lateral as well as in vertical direction. Below a certain sparsity ME-norms become insensitive for variations in wavelet dispersion and, hence, MEVA will not work.

Since inversion of $c \circ m$ m o $n$ of $\mathrm{f}$ e $t$ data can be described by a spatial wavelet $\mathrm{U}_{\mathrm{CO}}$ having similar properties as $\mathrm{U}_{\mathrm{ZO}}$, MEVA can as we11 be applied to inverted common offset data.

In subsection II.3.1, the analogy was indicated between the spatial wavelets describing a hyperbolic zero-offset diffractor response and, on the other hand, a hyperbolic $c \circ \mathrm{m}$ o $\mathrm{n}$ m $\mathrm{i} d \mathrm{p}$ o $\mathrm{n} t$ reflector response. This analogy 

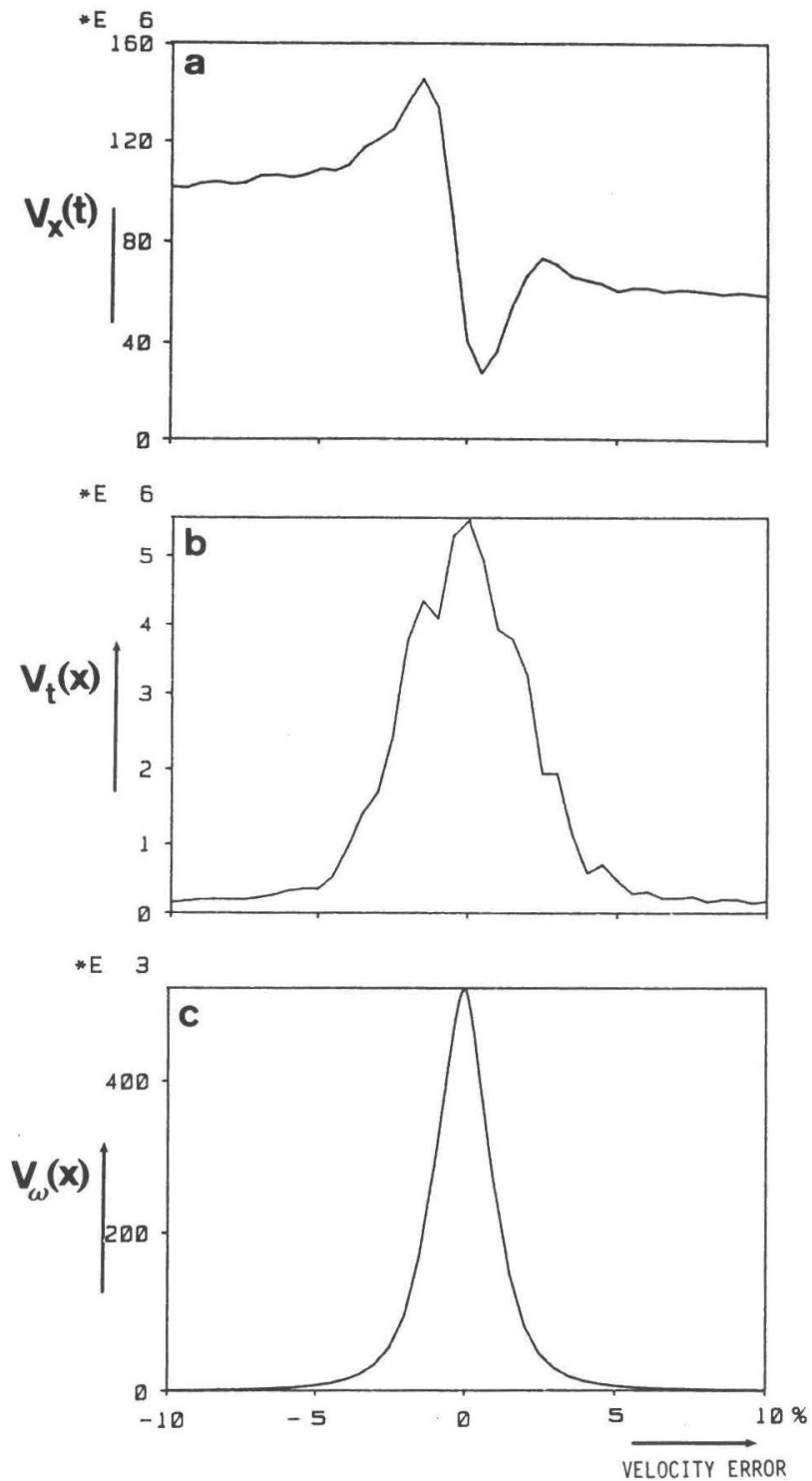

Figure V-10: ME-norms V, as a function of relative velocity errors, applied to an inverted diffractor response, calculated in three different ways:

a: $V_{x}(t)$ : in space-time domain along time axis

$b: v_{t}(x)$ : id., along space axis

c: $V_{\omega}(x)$ : in space-frequency domain along space axis amplitude parameter: $a=|y|,\left|U_{\mathrm{ZO}}\right|$ entropy function : $F(q)=q^{4}$ 
holds after inversion, and for velocity analysis purposes $\mathrm{U}_{\mathrm{ZO}}$ and $\mathrm{U}_{\mathrm{CMP}}$ may be interchanged. Hence, MEVA can also be applied to inverted CMP responses from a distribution of reflectors - i.e. largely continuous layer boundaries - to be written in the form:

$$
{ }_{\mathrm{CMP}}\left(\mathrm{x}, \Delta \tau_{\mathrm{m}}, \omega_{i}\right),
$$

where $\Delta \tau_{\mathrm{m}}$ represents the two-way zero-offset travel time to the $\mathrm{m}^{\text {th }} \mathrm{reflec}^{-}$ tor. In this way, MEVA forms an alternative to other velocity analysis techniques concerning CMP reflection data, see subsection I.4.2. It should be noted that MEVA can be applied to a multiple set of CMP gathers without the necessity to form one gather by stacking.

In the next chapter, several applications of MEVA to simulated as well as measured data sets will be evaluated.

\section{V.3.2 Velocity analysis using minimum entropy norms in the space-time domain}

The question arises if MEVA is also possible on inverted results in the $\mathrm{s}$ p a c e - $t \mathrm{i} \mathrm{m} e$ domain. In Fig. III-5 it was seen how the lateral extension (dispersion) of a diffractor image $i$ n creases with $i$ n creasing velocity error. Hence, consistently with what we did in the space-frequency domain, velocity information may be obtained by applying ME-norms to the data in lateral direction, i.e. to the $t r$ a $n s$ p o s e d data sets in the space-time domain. Doing so, we may expect a d e creasing ME-norm value for $i$ creasing velocity error, since the number of significant data points in the transposed traces increases and hence the resolving power decreases with increasing velocity error.

Fig. V-10 compares multi-trace(-channe1) ME-norms V applied to the inverted diffractor response corresponding to the geometry of Fig. V-7a, as a function of relative velocity error, calculated in three different ways: $V_{x}(t)$ in the space-time domain along the time axis (Fig. V-10a), $V_{t}(x)$ in the space-time domain along the space axis (Fig. $V-10 b$ ) and $V_{\omega}(x)$ in the space-frequency domain along the space axis (Fig. V-10c, identical to Fig. V-8d). Since vertical dispersion is not basically affected by velocity errors, $V_{x}(t)$ does not show a clear correlation with velocity error. As expected, both $V_{t}(x)$ and $V_{\omega}(x)$ have maximum value for correct velocity. Due to the orthogonality of the spectral 
components, the detectability of $v_{\omega}(x)$ is higher than that of $v_{t}(x)$. Therefore, $v_{\omega}(x)$ will generally prevail above $v_{t}(x)$ in velocity analysis techniques.

\section{V.4 CONCLUSIONS}

We have shown that application of minimum entropy norms to spatially inverted data forms an attractive tool for velocity analysis. MEVA should preferably be applied in the space-frequency domain but can also be used in the space-time domain after data transposition.

MEVA is applicable to inverted zero-offset or stacked data, where it is used to extract velocity information especially from diffraction data. This application can be extended to inverted common offset data.

Moreover, it was shown that MEVA can also be applied to inverted common midpoint reflection data, thus forming an interesting alternative to existing CMP velocity analysis techniques. 


\section{CHAPTER VI:}

\section{APPLICATIONS}

\section{VI.1 INTRODUCTION}

In this chapter, Minimum Entropy Velocity Analysis (MEVA) will successively be applied to media which have been selected from the field of echo-acoustical technology where velocities play an important role: seismics and medical diagnostics. Since in non-destructive material testing the measurement techniques are still analog and one-dimensional, applications in this field are not included here. Since the MEVA concept has primarily been developed in order to extract velocity information from diffraction energy, we first consider the applicability of MEVA to simulated diffractor distributions.

The chapter concludes with the formulation of practical recommendations for MEVA application.

\section{VI.2 SIMULATED ZERO-OFFSET DATA FROM DIFFRACTOR DISTRIBUTIONS}

\section{VI.2.1 Distributions of elementary diffractors}

First, we discuss the results of MEVA applied to the diffractor distribution earlier represented in Fig. II-10: 64 diffractors are randomly distributed in a homogeneous bulk medium (velocity $c=2000 \mathrm{~m} / \mathrm{s}$ ) below a line aperture, see Fig. VI-la for geometrical details. The noise-free zero-offset response at the aperture (Fig. VI-1b) has been simulated using a source pulse with a cosinewindowed amplitude spectrum between 0.5 and $3 \mathrm{MHz}$. Hence, the average diffrac-

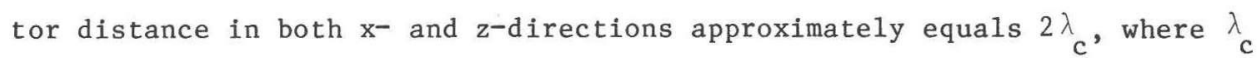




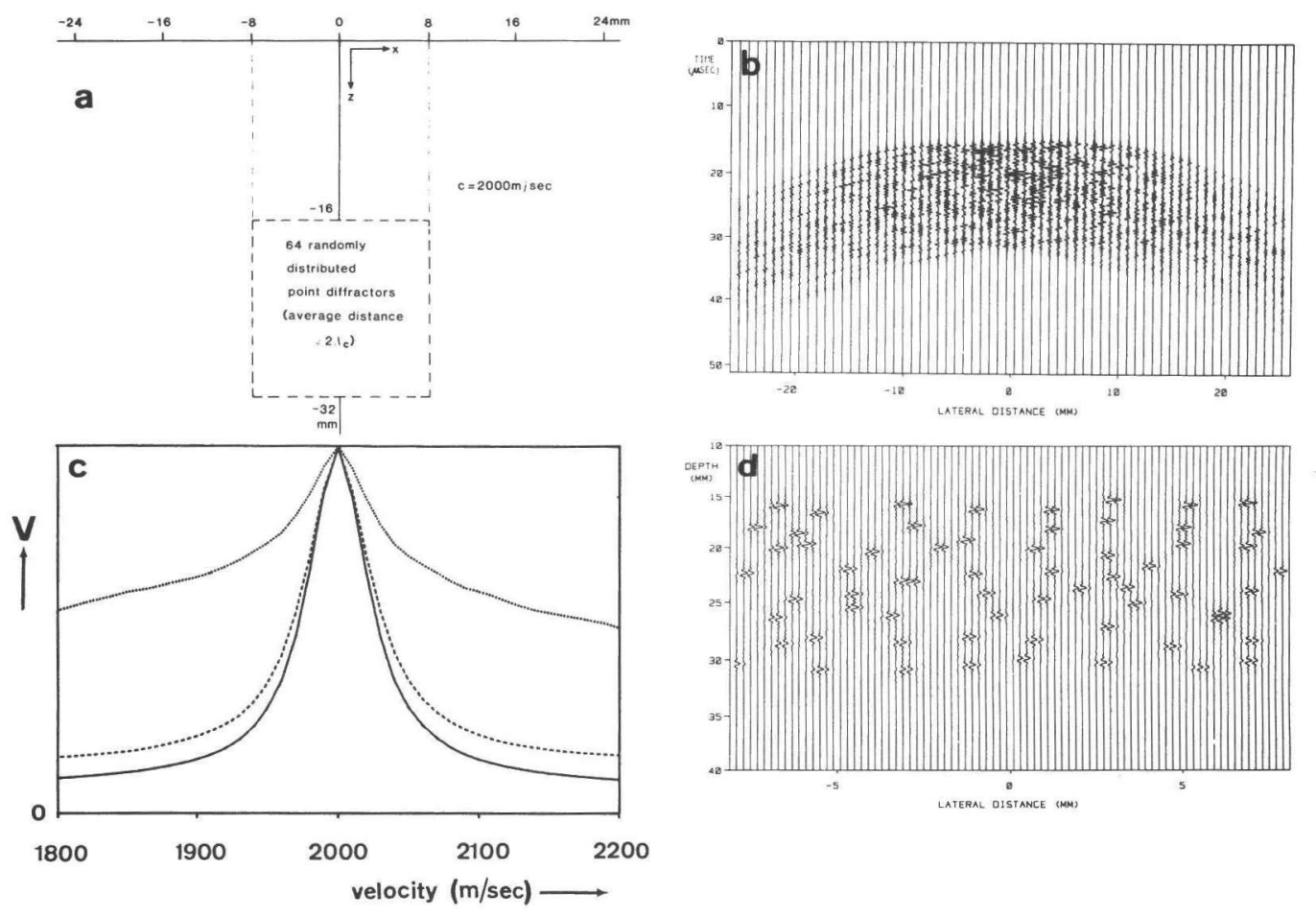

Figure VI-1: MEVA applied to a configuration of 64 diffractors random$1 y$ distributed in a homogeneous medium. Average diffractor distance in $x$ - and $z$-directions is approx. $2 \lambda_{c}, \lambda_{c}$ being the central wavelength of the source pulse.

a. geometry

b. zero-offset response

c. ME-norms as a function of velocity error

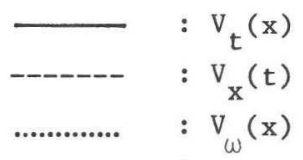

d. image obtained with correct velocity 
represents the wavelength of the central component of the source pulse spectrum.

Fig. VI-1c shows the results of MEVA applied to the inverted response. The downward extrapolation velocity has been varied between 1800 and $2000 \mathrm{~m} / \mathrm{s}$. Respectively, the three curves represent, as a function of velocity, ME-norm $v_{t}(x)$ calculated in the space-time domain along the space axis, $v_{x}(t)$ calculated in the space-time domain along the time axis, and $V_{\omega}(x)$ calculated in the space-frequency domain along the space axis. In all examples in this chapter, amplitude parameter $a=y^{2}$ and entropy function $F(q)=q^{4}$ have been applied.

It is seen that application of MEVA to a two-dimensional diffractor distribution leads to results which differ from what we found earlier for a single diffractor:

(1) ME-norms as calculated in the three ways considered, i.e. $v_{t}(x), v_{x}(t)$ and $\mathrm{V}_{\omega}(\mathrm{x})$, all show a maximum value for correct velocity. Inversion with this velocity value yields the optimally resolved image shown in Fig. VI-1d (identical to Fig. II-10d).

(2) $v_{t}(x)$ and also $v_{x}(t)$ have a higher velocity detectability than $v_{\omega}(x)$.

These phenomena can be explained as follows.

ad_- $(1)$ :

As discussed before, introduction of velocity errors in the inversion operator causes increasing lateral extension of each diffractor image. If the diffractors have different vertical coordinates, increasing lateral extension also causes an increasing number of 'significant' data points in each time (or depth) trace and, hence, increasing entropy along those traces. This is schematically illustrated in Fig. VI-2, and can also be clearly seen in Fig. III-7 where the image of the present diffractor distribution is displayed for relative velocity errors of $-10 \%$ and $+10 \%$.

Hence, for two-dimensional diffractor distributions as considered here, entropy along the time axis as well as along the space axis is minimum in case of correct velocity, yielding maximum values for $v_{x}(t)$ as well as $v_{t}(x)$. 


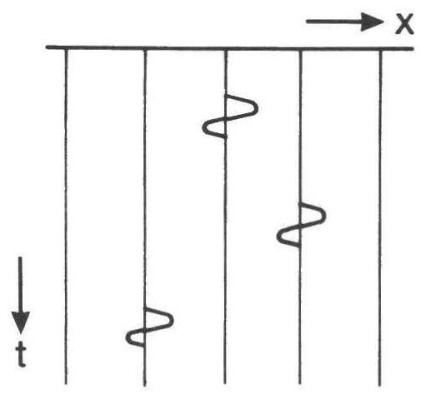

a

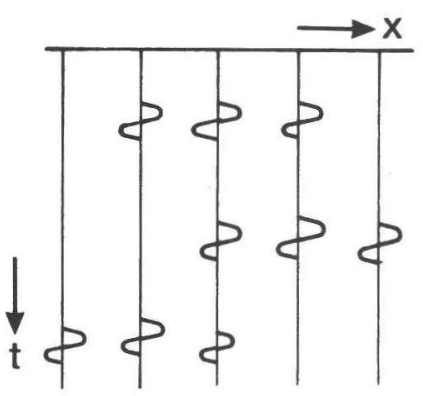

b

Figure VI-2: Inverted result (schematic) of a two-dimensional diffractor distribution.

a. correct velocity: minimum entropy in $x^{-}$and t-directions

b. incorrect velocity: increased entropy in $x^{-}$and $t$-directions

a-d_- 2 ):

In calculating ME-norm $V_{t}(x)$, dispersion effects in each transposed time trace are considered separately. However, in calculating $\mathrm{V}_{\omega_{0}}(\mathrm{x})$ for some spectral component $\omega_{0}$, total spectral strength for $\omega_{=} \omega_{0}$ is considered as a function of lateral coordinate $x$. Since only the a in $p 1 i t$ u d e spectrum is taken into account, the depth from which a contribution originates (or the corresponding time delay) is of no importance in the calculation. This means that the three schematic patterns of diffractor images given in Fig. VI-3 yield identical amplitude distributions $\left|\mathrm{P}\left(\mathrm{x}, \omega_{0}\right)\right|$ for spectral component $\omega_{0}$ and, hence, identical values of $\mathrm{V}_{\omega_{0}}(\mathrm{x})$.

It should be realized that the pattern of Fig. VI-3b may represent the optimal image of a distribution of 5 diffractors. Inversion of the same configuration with an erroneous velocity may - apart from hyperbolic curvature and amplitude effects - lead to an approximation of the pattern of Fig. VI-3c. Hence, large differences in $V_{\omega}(x)$ for correct and erroneous velocity values are not to be expected for such configurations.

Generally, the phenomenon illustrated in Fig. VI-3 can be formulated as follows: in calculating ME-norm $\mathrm{V}_{\omega}(\mathrm{x})$, a two-dimensional diffractor distribution is interpreted as a o n e-dimensional distribution, see Fig. VI-3a. The lateral sparsity of this one-dimensional distribution can be significantly smaller than the lateral sparsity for each depth level in the original twodimensional distribution. This leads to decreasing sensitivity for variations in lateral dispersion, i.e. for velocity errors. 


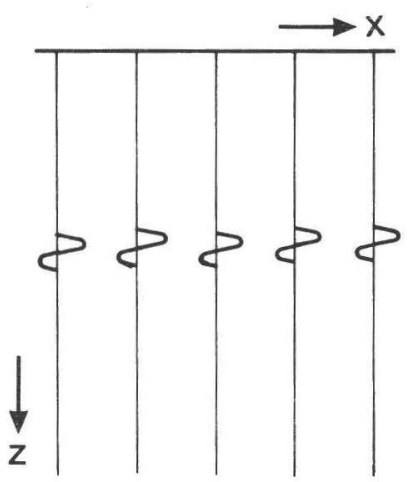

a

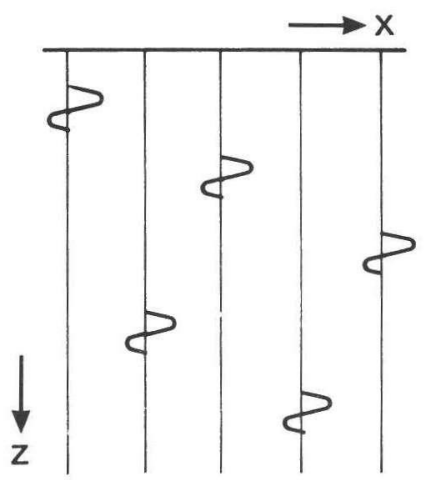

b

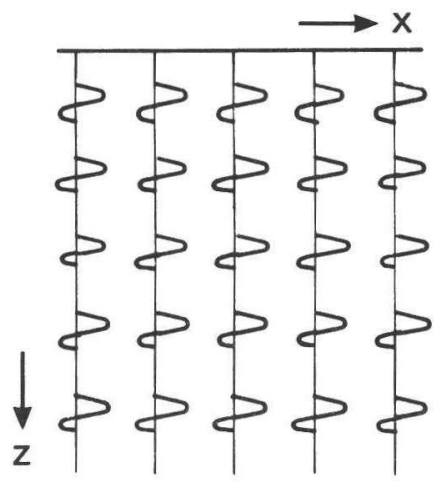

C

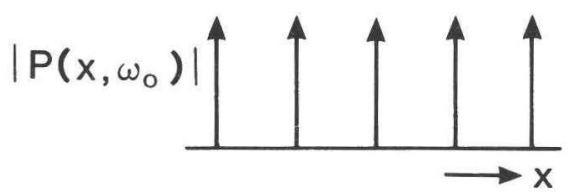

d

Figure VI-3: Three diffractor image configurations ( $a, b, c)$ yielding the same amplitude distribution for spectral component $\omega_{0}(d)$ and identical values of $M E-n o r m V_{\omega_{0}}(x)$.

For the diffractor distribution considered in Fig. VI-1, the above argument is illustrated in Fig. VI-4. For central spectral component $\omega_{c}$ the amplitude distribution $\left|P\left(x, \omega_{c}\right)\right|$ is given for correct downward extrapolation velocity (Fig. VI-4a) and a relative velocity error of $10 \%$ (Fig. VI-4b). It is seen that both patterns have a high-entropy character. Particularly, the dispersion at the $\mathrm{e} d \mathrm{~g}$ e $\mathrm{s}$ of the diffractor area shows significant dependence of the applied velocity value, causing the peak of $V_{\omega}(x)$ to occur at the correct velocity as shown in Fig. VI-lc. 


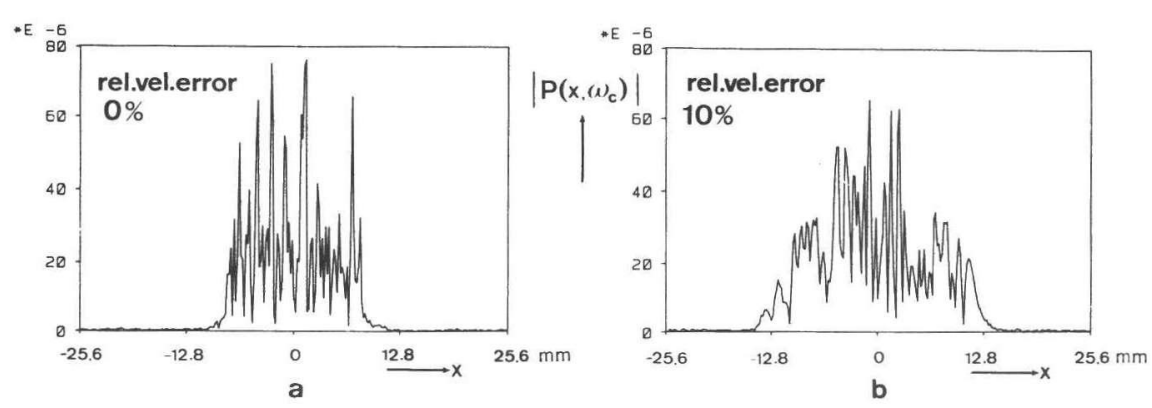

Figure VI-4: Amplitude distribution $\left|\mathrm{P}\left(\mathrm{x}, \omega_{\mathrm{c}}\right)\right|$ of the diffractor distribution of Fig. VI-1 for central spectral component $\omega_{c}$. a. correct velocity b. $10 \%$ relative velocity error

Conclusions:

(1) For two-dimensional $(x, z)$ diffractor distributions, MEVA can successfully be applied in the space-time domain (transposed a n d non-transposed) as well as in the space-frequency domain. Considering efficiency, application in the space-time domain is attractive, since Fourier transformation can be omitted.

(2) For two-dimensional diffractor distributions, the prevalence of $v_{\omega}(x)$ over $v_{t}(x)$ and $v_{x}(t)$ disappears, since decrease of 'effective' lateral sparsity can lead to decreasing sensitivity to velocity errors.

In the previous simulation example the average diffractor distance was approximately twice the central wavelength of the source pulse. A similar simulation experiment on MEVA application has been done for the two-dimensional diffractor configuration shown in Fig. VI-5a, where the average distance in both directions has been reduced to the central wavelength of the pulse. Note that in contrast to the previous case where dimensions and frequencies as common in the field of medical diagnostics were used, we now consider seismic ranges: the aperture size is $2400 \mathrm{~m}$, the source spectrum contains frequencies between 10 and $50 \mathrm{~Hz}$. The bulk medium velocity has been chosen $1500 \mathrm{~m} / \mathrm{s}$.

ME-norm $v_{t}(x)$ - which also for this configuration proves to have the highest detectability - has been calculated as a function of velocity in three different ways: 

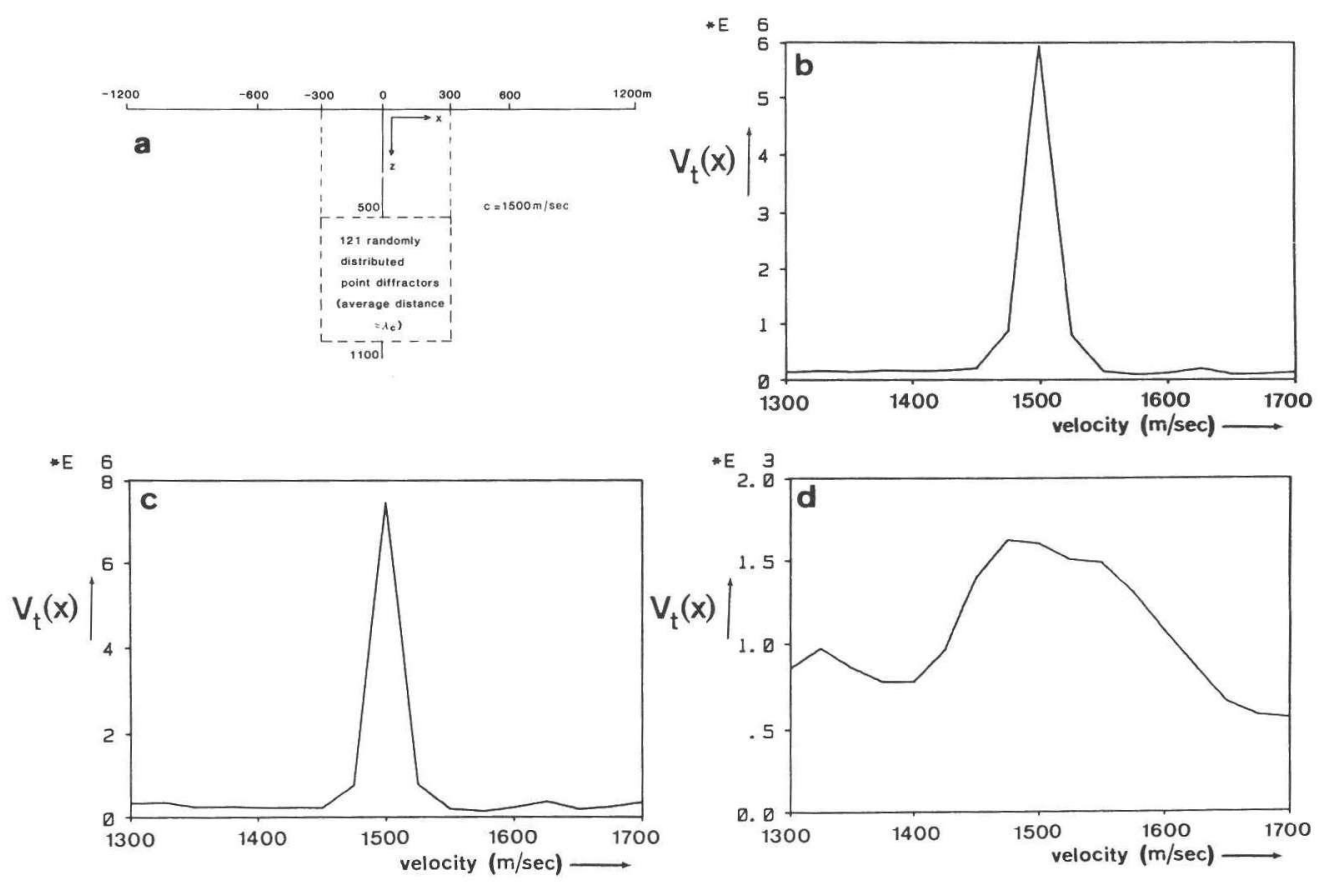

Figure VI-5: MEVA applied to a configuration of 121 diffractors random1y distributed in a homogeneous medium. Average diffractor distance in $x$ - and $z$-directions is approx. $1 \lambda_{c}, \lambda_{c}$ being the central wavelength of the source pulse.

a. geometry

b. ME-norm $V_{t}(x)$ as a function of velocity; no windowing

c. id., with windowing after inversion

d. id., with windowing before inversion 
(1) inversion of the complete zero-offset response and ME-norm calculation on the complete inverted data set (Fig. VI-5b),

(2) inversion of the complete response and ME-norm calculation on a limited part of the inverted data (windowing after inversion, Fig. VI-5c),

(3) inversion of a limited part of the response and ME-norm calculation over the inverted result (windowing before inversion, Fig. VI-5d).

The applied windows are indicated in Fig. VI-5a.

It is seen that

(1) application of MEVA after inversion of the complete response yields an accurate detection of the correct velocity,

(2) windowing of the inverted data - i.e. neglection of the inverted diffraction phenomena at the edges of the diffractor area - has no significant influence,

(3) windowing before inversion eliminates a useful amount of recorded diffraction energy and, hence, deteriorates the velocity detectability.

Additional simulation experiments have been performed on diffractor distributions with average distances significantly smaller than the central wavelength of the source pulse. In accordance with the general assumption that one wavelength forms the limit of spatial resolution (see also Fig. VI-5), MEVA fails to provide information for these situations.

Conclusionions:

(1) MEVA yields accurate velocity detection for two-dimensional diffractor distributions with average distances (in both directions) of at least the central wavelength of the source pulse.

(2) Windowing $b$ e $f \circ r$ e inversion may significantly deteriorate the results of MEVA, whereas windowing a $\mathrm{f}$ e $r$ inversion does not have a significant influence.

\section{VI.2.2 Diffracting discontinuities on reflectors}

We now consider the configuration earlier represented in Figs. I-1 and II-11, consisting of a finite reflector with a central opening. The width of the opening is approximately 1.5 times the central wavelength of the source pulse. The propagation velocity of the surrounding medium equals $2000 \mathrm{~m} / \mathrm{s}$. See Fig. VI-6a for more geometric details. It should be noted that the present configuration is relevant for seismic applications, where discontinuous layer boundaries are considered. 


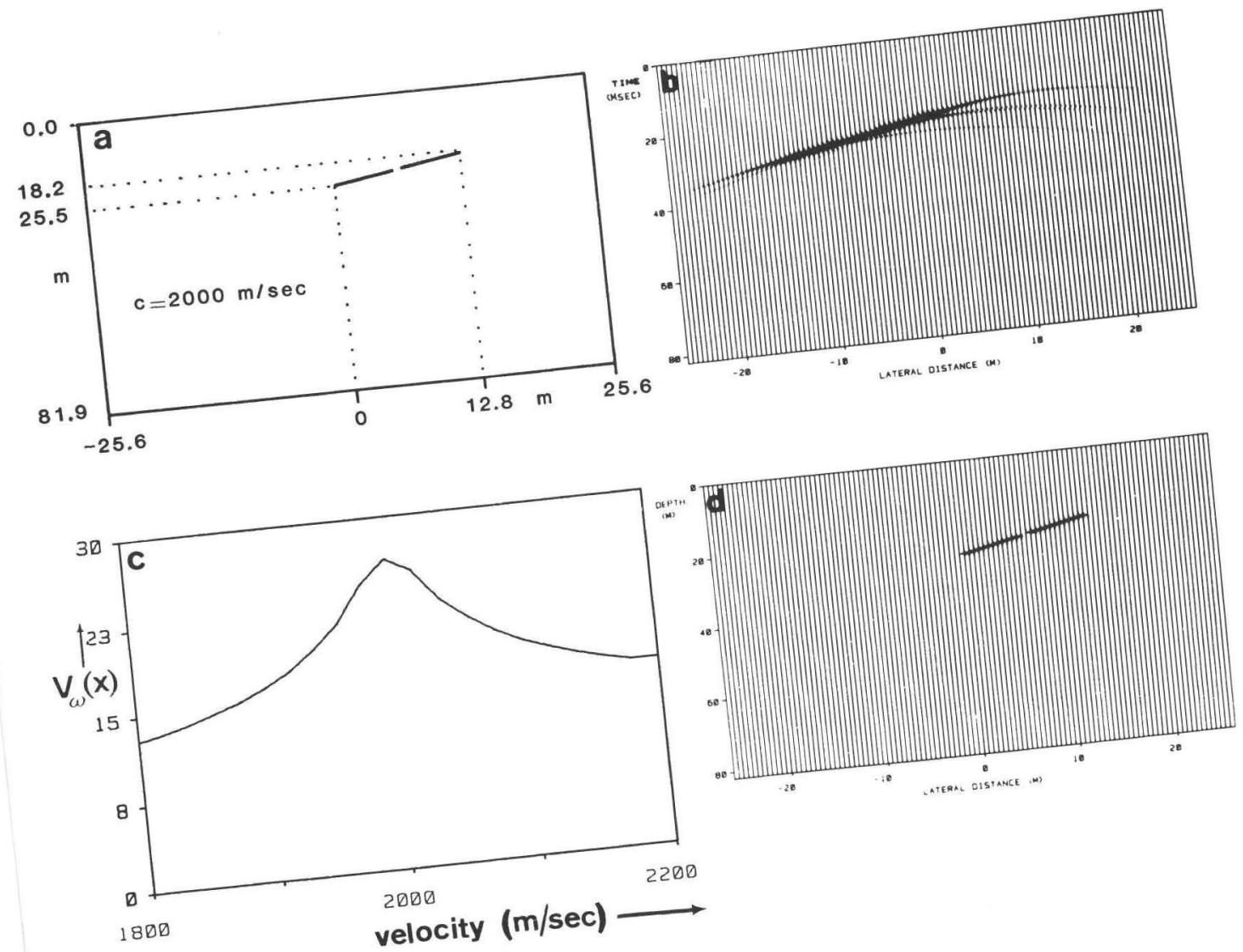

wire VI-6: MEVA applied to finite reflector with a central opening. Opening width: approx. $1.5 \lambda_{c}\left(\lambda_{c}\right.$ is the central wavelength of the source pulse).

a. geometry

b. zero-offset response

c. $M E-n o r m V_{\omega}(x)$ as a function of velocity

with correct velocity

d. image 
The continuous parts of the reflector may be considered as an array of elementary diffractors with zero interval width, in digital processing techniques approximated by a finite distance determined by the spatial sampling interval. In modeling the zero-offset response of such a continuous diffractor array, the hyperbolic responses of the individual diffractors interfere such that no diffraction phenomenon - in the sense of scattering - appears. At lateral discontinuities like edges and other types of significant reflectivity changes this interference is incomplete, so that only such points effectively appear as secondary sources of diffraction energy. This is illustrated in Fig. VI-6b, where the noise-free zero-offset response of the model described in Fig. VI-6a is shown: four diffraction curves are clearly seen, generated by the four discontinuities in the model.

Hence, for MEVA application the above model is highly equivalent to a sparse configuration of four diffractors. However, in the zero-offset response the 'useful' diffraction energy is low in respect to the 'useless' reflection energy, which in the MEVA context is equivalent with a low signal-to-noise ratio. As expected on the above arguments, results of MEVA qualitatively resemble those for a single diffractor: $V_{x}(t)$ fails to give any velocity information, whereas $V_{t}(x)$ and $V_{\omega}(x)$ show a peak value for correct velocity. $\mathrm{V}_{\omega}(\mathrm{x})$ has a better detectability than $\mathrm{V}_{t}(\mathrm{x})$, since the 'overall' lateral dispersion of the inverted pattern is taken into account in $v_{\omega}(x) \cdot V_{\omega}(x)$ is given as a function of velocity in Fig. VI-6c, the image obtained with correct velocity being displayed in Fig. VI-6d. The low signal-to-noise ratio of diffraction energy due to the abundant amount of reflection energy suggests that better MEVA results are to be expected after preprocessing such that most reflection energy is removed from the data. Then, as for the two-dimensional diffractor distributions discussed in subsection VI.2.1, MEVA is expected to work satisfactorily in the space-time domain as well.

\section{Conclusions:}

(1) In zero-offset responses from highly continuous reflectors, as found in layered media, only the contributions from lateral discontinuities and other types of large lateral reflectivity changes appear in the form of diffraction energy.

(2) Hence, for MEVA application such layered media are equivalent with diffractor distributions, the sparsity of which is determined by the geometry of the 1ateral reflectivity discontinuities. 
(3) In the zero-offset response of layered media with sparse diffraction energy sources, the ratio between diffraction energy and reflection energy is often low, which in the MEVA context is equivalent with a low signal-tonoise ratio. This suggests that MEVA results should improve after preprocessing such that most reflection energy is removed from the data.

(4) Applying MEVA to zero-offset data from layered media with sparse diffraction energy sources, ME-norm $V_{\omega}(x)$ gives optimal velocity detectability. However, it is expected that MEVA will also yield satisfactory results in the space-time domain after preprocessing such that reflection energy is eliminated.

\section{VI.3 SEISMIC APPLICATIONS}

\section{VI.3.1 Simulated CMP data from one horizontal boundary}

In subsection V.3.1, the applicability of MEVA to common midpoint reflection data was indicated, based on the analogy of the spatial wavelets describing propagation in a CMP reflector response and a zero-offset diffractor response. Hence, all arguments and conclusions given in section V.3 for application of MEVA to the zero-offset response of a single diffractor also hold for the CMP response of a horizontal boundary. It was suggested that MEVA forms an alternative to existing CMP velocity analysis techniques.

To support this suggestion, we here compare the result of MEVA with the result of the well-known velocity analysis technique described by Taner and Koehler (1969), expressing for each velocity estimate the coherency of the normal moveout corrected data in terms of 'semblance' $E$. The comparison is made for simulated CMP data from a horizontal boundary below a homogeneous layer ( $c=$ $1500 \mathrm{~m} / \mathrm{s}$ ) at a depth of $500 \mathrm{~m}$, the maximum half offset being $768 \mathrm{~m}$. The noisefree situation as well as a signal-to-noise ratio of $0 \mathrm{~dB}$ in the response has been considered. The source pulse used has a cosine-windowed amplitude spectrum between 10 and $60 \mathrm{~Hz}$.

Fig. VI-7 shows the results. It is seen that ME-norm $V_{\omega}(x)$ - with amplitude parameter $a=y^{2}$ and entropy function $F(q)=q^{4}$ - yields a significantly better velocity detectability than semblance $E$ for the noise-free situation. For the situation with noise, the detectability of the two norms is approximately equal. Further experiments have shown that for entropy functions $F(q)$ containing a lower power of $\mathrm{q}$, the prevalence of the ME-norm disappears. On the other 

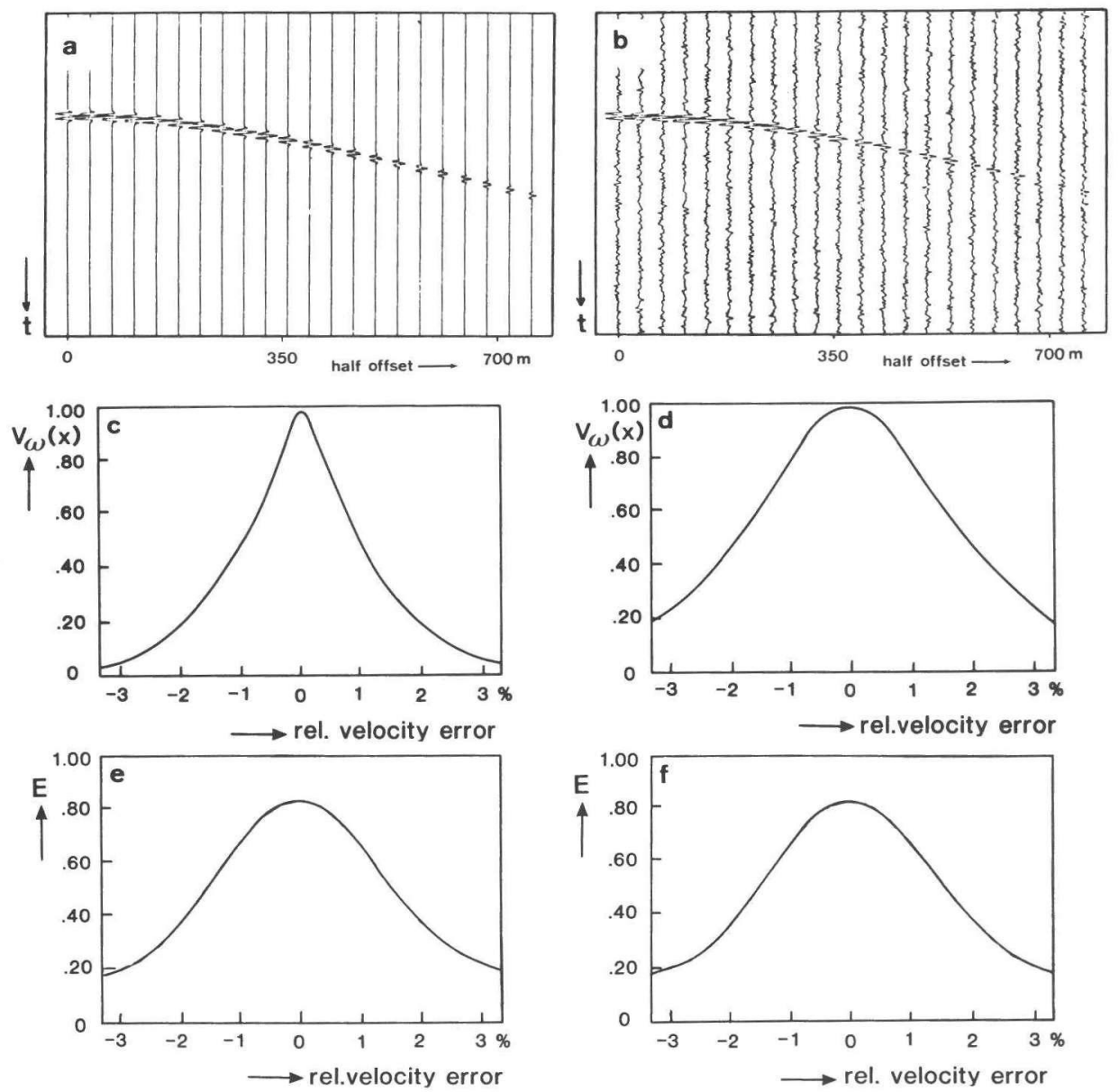

Figure VI-7: Comparison of MEVA with velocity analysis based on coherency.
a. CMP response from a horizontal layer, noise-free
b. CMP response, id., S/N-ratio $0 \mathrm{~dB}$
c. ME-norm $V_{\omega}(x)$ as a function of velocity for response a
d. id., for response b
e. semblance $E$ as a function of velocity for response a
f. id., for response b 
hand, for higher power values of $\mathrm{q}$ in the entropy function, ME-norms become too sensitive for occasional (e.g. noise) peaks, so that $F(q)=q^{4}$ seems an appropriate choice for velocity analysis applications.

\section{Conclusions:}

(1) MEVA is a good alternative to existing CMP velocity analysis techniques.

(2) Application of ME-norms $V_{\omega}(x)$ using a strong entropy function yields a better velocity detectability than application of coherency techniques. $\mathrm{F}(\mathrm{q})=\mathrm{q}^{4}$ appears to be an appropriate choice.

\section{VI.3.2 Simulated CMP data from a mode1 of three dipping layers}

Fig. VI-8a shows the model considered, consisting of three dipping 1ayers with velocities $c_{1}=2000 \mathrm{~m} / \mathrm{s}, c_{2}=2500 \mathrm{~m} / \mathrm{s}$ and $c_{3}=3000 \mathrm{~m} / \mathrm{s}$. The raypath pattern for CMP modeling is drawn in the model. Fig. VI-8b shows the CMP response with a signal-to-noise ratio $0 \mathrm{~dB}$, in which three hyperbolic reflection patterns can be distinguished. The source pulse has a cosine-windowed amplitude spectrum between 10 and $70 \mathrm{~Hz}$.

An estimate of the stack of the three boundary responses is now obtained by successively migrating the CMP gather, in a non-recursive way, with a number of velocity values spanning a range within which the three stacking velocities are to be expected. For each velocity value, MEVA is applied to the inverted data such that $V_{\omega}(x)$ is calculated separately within three time windows. The windows are chosen such that in each window the stack estimate of one boundary response is to be expected. The zero-offset travel times in the CMP gather yield information for proper window choice. In Figs. VI-8d, f,h the three windows are indicated.

Figs. VI-8c,e,g show the values of $V_{\omega}(x)$ as a function of velocity within the three windows. The velocity value corresponding with the maximum of $v_{\omega}(x)$ within window $n(n=1,2,3)$ is interpreted as an estimate of the stacking velocity $v_{s t, n}$ for the CMP response of the $n^{\text {th }}$ boundary. Fig. VI-8d, $f, h$ show the migrated results obtained with the three stacking velocity estimates. It is seen that

(1) within each window, a well-resolved stack estimate is obtained for the corresponding boundary response, using the stacking velocity estimate found with MEVA,

(2) the focussing quality of the stack estimates in terms of lateral 

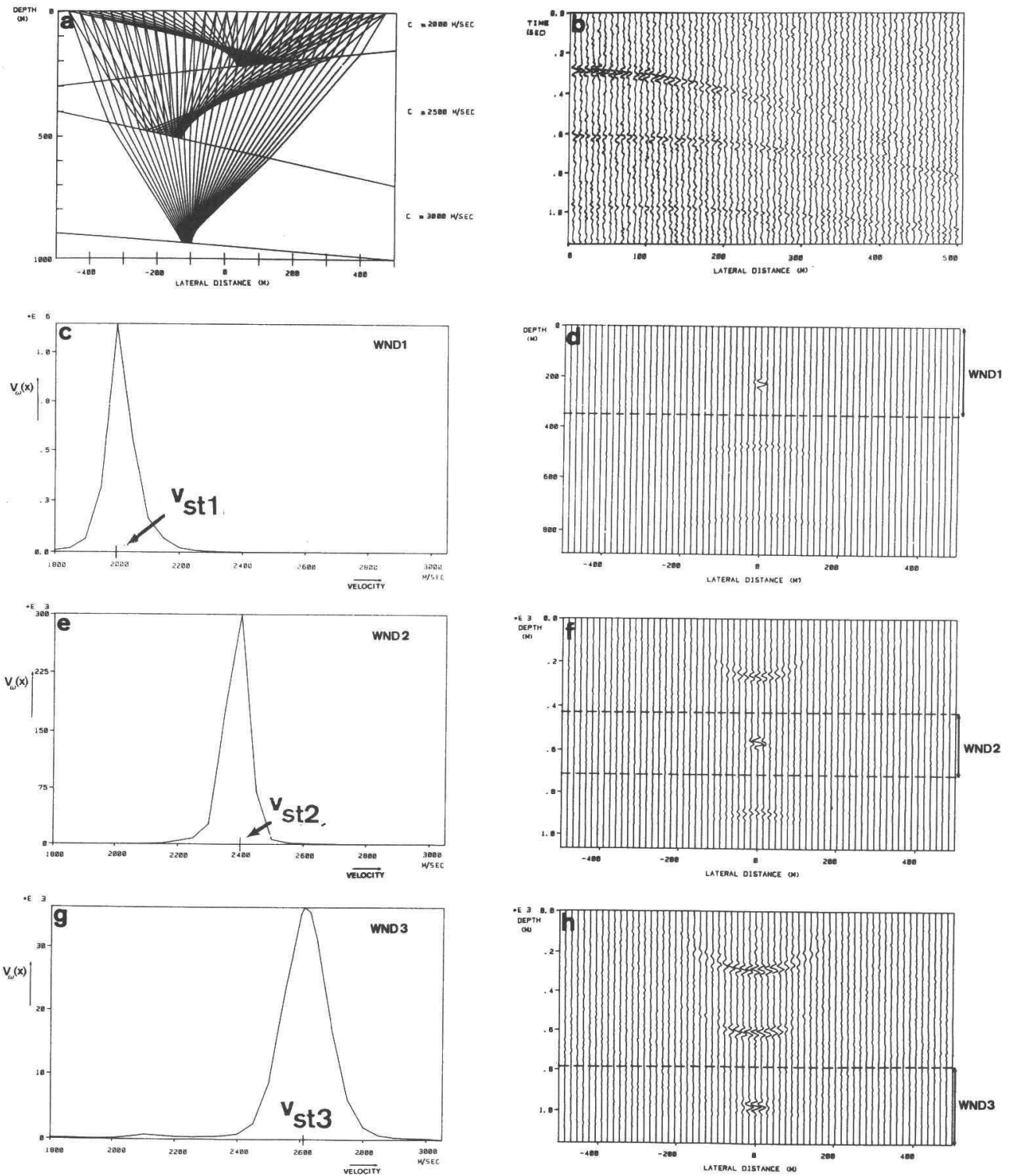
Figure VI-8: MEVA applied to three dipping layers.

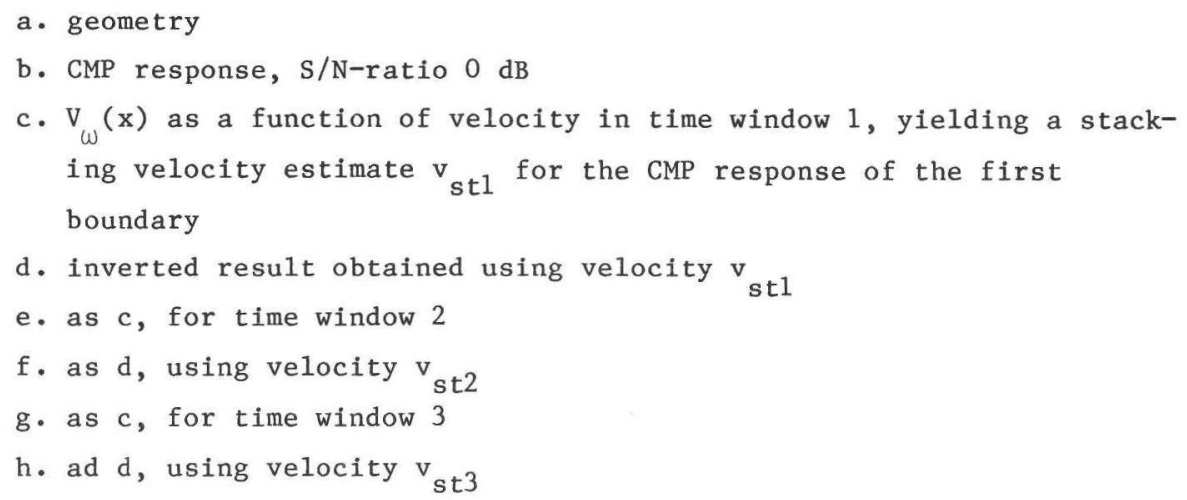



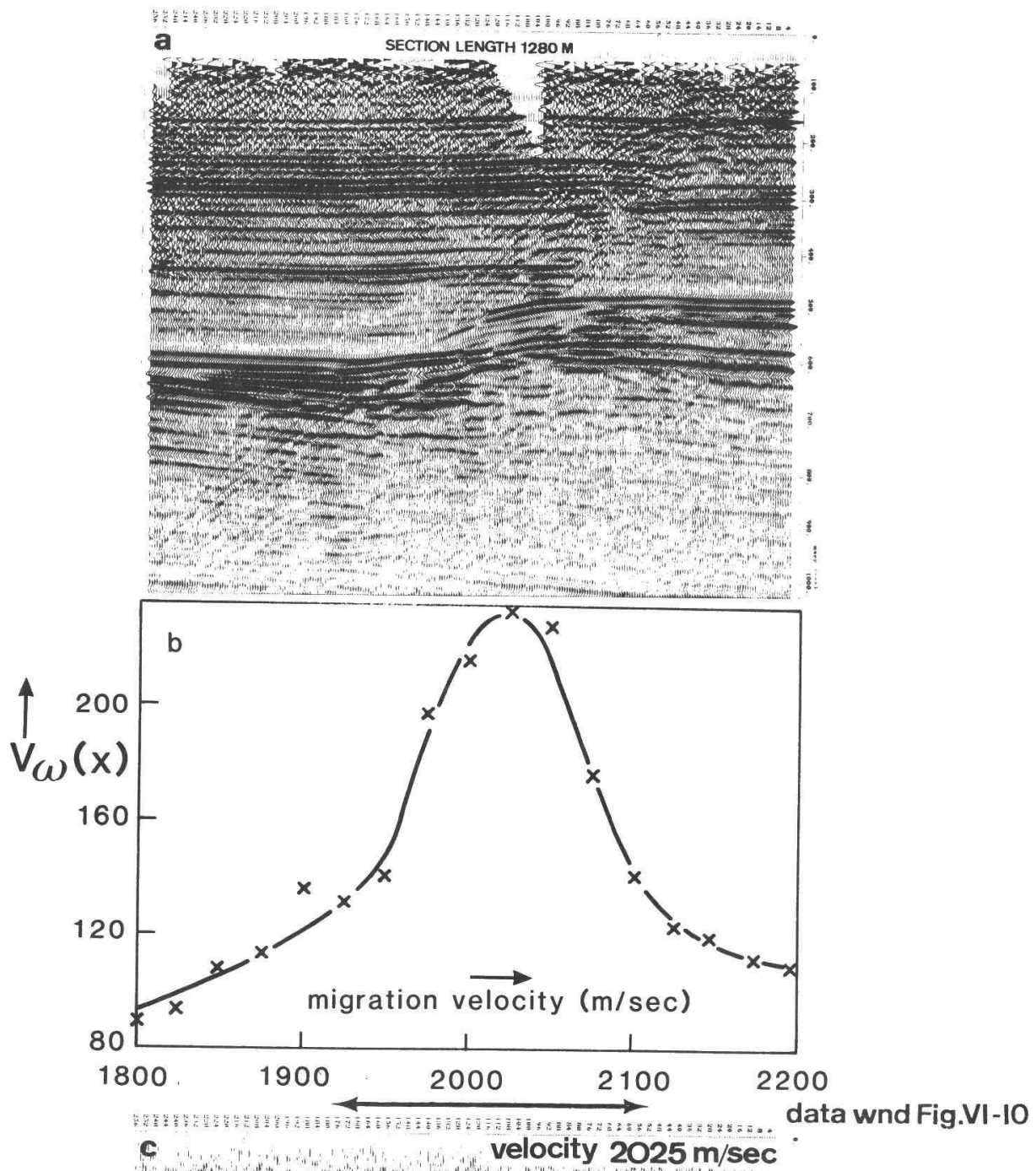

c

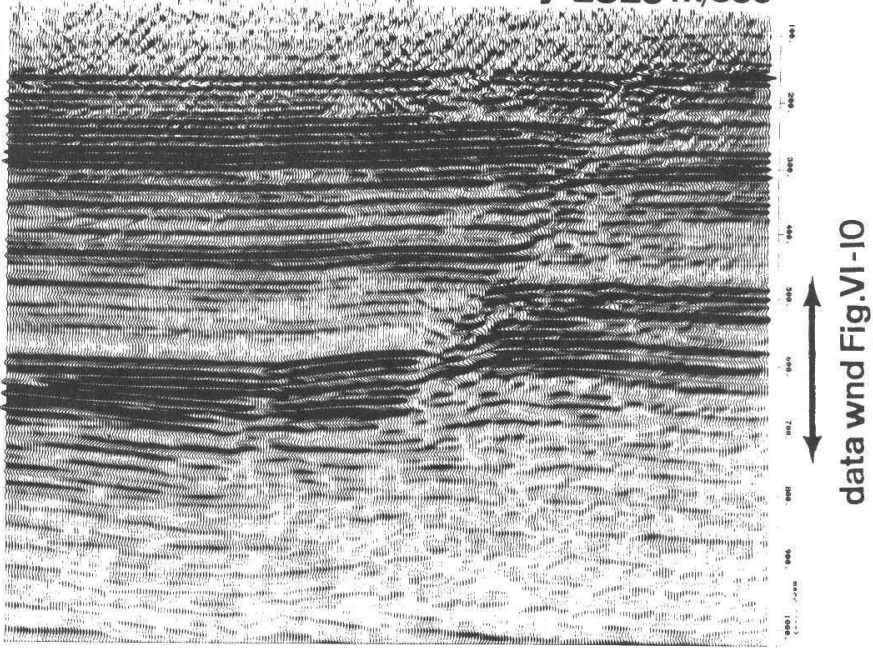



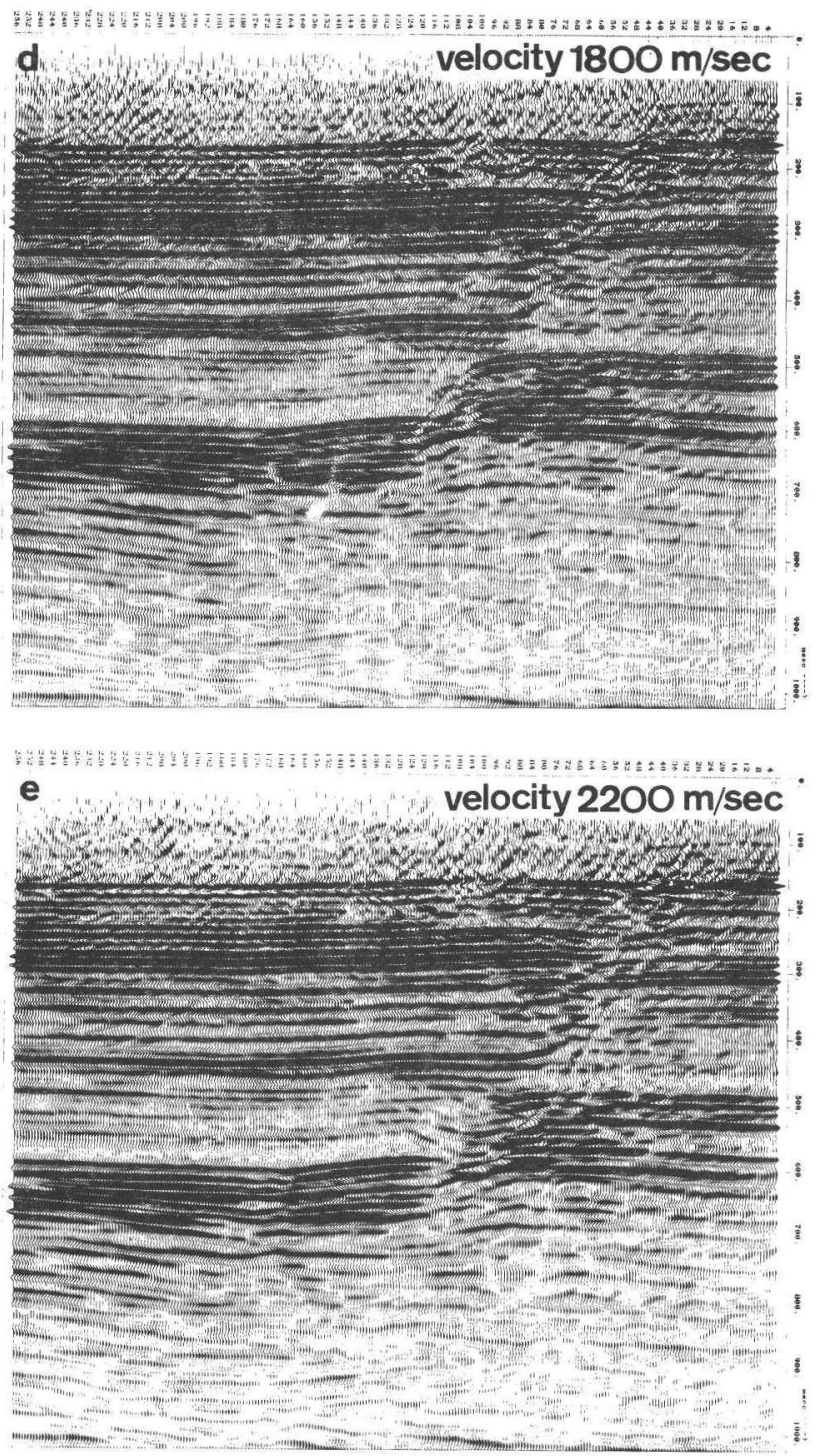

Figure VI-9: MEVA applied to stacked field data

a. stacked data

b. $v_{\omega}(x)$ as a function of velocity, calculated for the full inverted data set

c. migrated result obtained with velocity $2025 \mathrm{~m} / \mathrm{s}$

d. id., obtained with velocity $1800 \mathrm{~m} / \mathrm{s}$

e. id., obtained with velocity $2200 \mathrm{~m} / \mathrm{s}$ 
'compactness' decreases with depth. This well-known phenomenon is caused by decrease of spatial bandwidth with depth.

Hubral (1976) gives a formula with which, for dipping layers, interval velocities can be estimated from stacking velocities. Applying this formula - which is an extension of the famous Dix-formula - the results given in table VI/l are obtained.

\section{Table VI/1}

Interval velocities calculated from stacking velocities estimated with MEVA, using Hubral's formula.

\begin{tabular}{|c|l|l|l|l|}
\hline 1ayer $\mathrm{n}$ & $\mathrm{v}_{\mathrm{st}, \mathrm{n}}(\mathrm{m} / \mathrm{s})$ & $\mathrm{c}_{\mathrm{n}}(\mathrm{m} / \mathrm{s})$ & $<\mathrm{c}_{\mathrm{n}}>(\mathrm{m} / \mathrm{s})$ & rel.error \\
\hline 1 & 2000 & 2000 & 2000 & $0 \%$ \\
2 & 2400 & 2500 & 2555 & $2 \%$ \\
3 & 2610 & 3000 & 3026 & $1 \%$ \\
\hline
\end{tabular}

It is seen that, for the present model where interval velocities increase with depth, these velocities are estimated with an accuracy of a few percents. This accuracy is of the same order as obtained with velocity analysis techniques based on coherency, see Taner and Koehler (1969).

\section{Conclusions:}

(1) In estimating stacking velocities from CMP gathers obtained from dipping layer configurations, MEVA is a successful alternative to velocity analysis using coherency techniques. This is confirmed by the equal accuracy with which interval velocities can be estimated from stacking velocities obtained with the two techniques.

(2) In order to estimate stacking velocities for successive boundary responses, MEVA must be applied to the inverted data within different time windows.

\section{VI.3.3 Stacked field data}

Fig. VI-9a shows a set of stacked field data in which a high amount of diffraction energy is clearly visible. Hence, this data set is very appropriate to determine optimal migration velocities using MEVA.

Fig. VI-9b shows ME-norm $\mathrm{V}_{\omega}(\mathrm{x})$ as a function of migration velocity, using the full migrated data set and the full spectrum of the source pulse for the 
ME-norm calculation. The maximum value of $v_{\omega}(x)$ is found for a velocity of $2025 \mathrm{~m} / \mathrm{s}$, yielding the migrated pattern of Fig. VI-9c where, due to focussing of the diffraction energy, different faults are clearly visible. Note that MEVA has indicated the velocity value which yields an optimal o v e r a 11 resolving power in lateral direction for the complete data set. For comparison, migrated patterns obtained with velocity values $1800 \mathrm{~m} / \mathrm{s}$ and $2200 \mathrm{~m} / \mathrm{s}$ are shown in Fig. VI-9d,e. It is seen that now the faults are imaged less sharply due to undermigration of diffraction energy in the former case and overmigration in the latter.

MEVA was also applied to the inverted data within a space-time window indicated in Fig. VI-9c. Then, $V_{\omega}(x)$ varies as a function of velocity as shown in Fig. VI-10a: a maximum is found for velocity value $2060 \mathrm{~m} / \mathrm{s}$. This means that for optimal migration (in the sense of optimal lateral resolution) of the subsurface area within the window, a higher velocity is required than for optimal migration of the full data set. Obviously, for the latter case also the lower interval velocities of the upper layers play a significant role.

In subsection V.3.1 it was suggested that processing time required for MEVA in the space-frequency domain can be reduced by considering only a few frequency components with high signal-to-noise ratio. For the data considered here, the source pulse has a spectrum roughly between 5 and $120 \mathrm{~Hz}$, with high energy components between 30 and $80 \mathrm{~Hz}$. Hence, MEVA was also applied to the inverted data within the above window for the latter frequency range only. Disappointing$1 y$, the technique now failed to work, and also when frequencies between 80 and $120 \mathrm{~Hz}$ were taken into account. A good result, however, giving a maximum of $v_{\omega}(x)$ for the same velocity value as in the broadband case with even higher detectability, was obtained when only the $1 \circ \mathrm{w}$ frequencies between 5 and $30 \mathrm{~Hz}$ were considered, see Fig. VI-10b. Even when only one low-frequency component $(15 \mathrm{~Hz})$ is included in the ME-norm calculation, MEVA yields a consistent result, see Fig. VI-10c.

The above effects of bandwidth limitation in ME-norm calculation can be explained by assuming that diffraction energy - generally containing strong highfrequency components - is $1 \circ \mathrm{w}-\mathrm{p}$ a s s filtered by the stacking process, due to destructive interference of high-frequency components during addition of the normal moveout corrected data. 

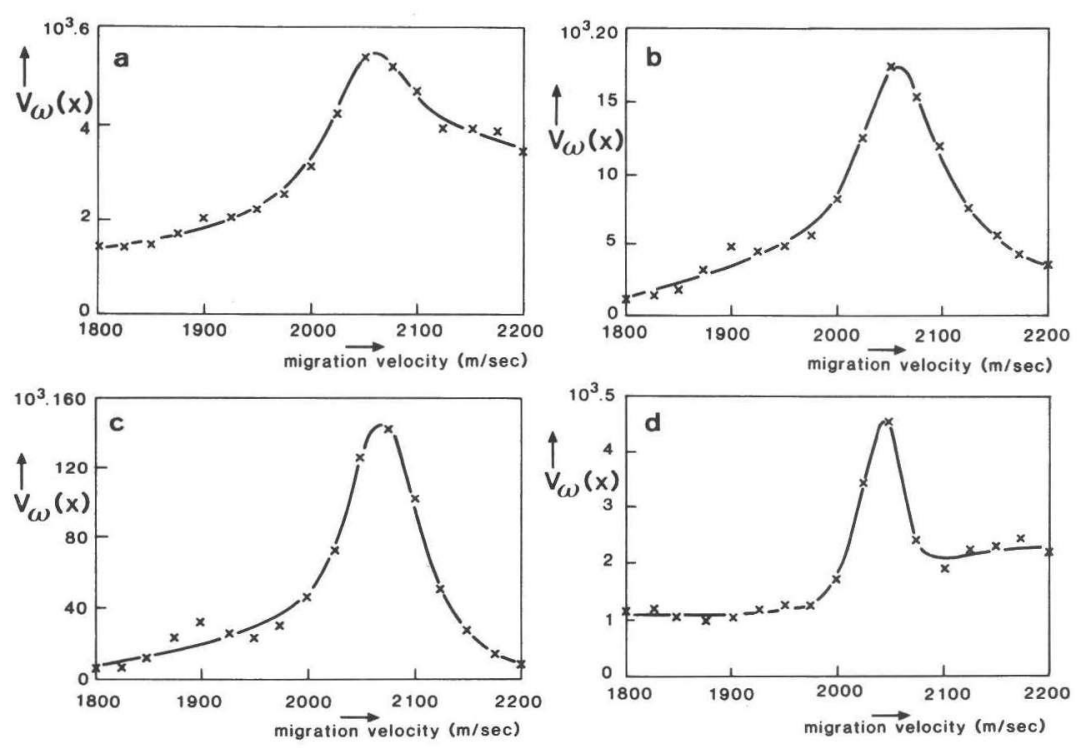

Figure VI-10: MEVA applied to a limited part of the inverted stacked data (for data window see Fig. VI-9c).

a. broadband result $(5-120 \mathrm{~Hz})$

b. result for $5-30 \mathrm{~Hz}$ frequency band

c. result for single frequency component $15 \mathrm{~Hz}$

d. result for corresponding near-trace data, $30-80 \mathrm{~Hz}$ frequency band

This assumption was confirmed by an additional experiment, where MEVA was applied to the $n$ e a $r-t r$ a $c$ e data of the present multi-trace data set, i.e. for each source position the normal moveout corrected recording of the nearest detector (common offset) is taken. With stacked data, near-trace data have in common that they approximate zero-offset data. A basic difference, however, is that addition of traces has not taken place so that - at the cost of a low signal-to-noise ratio - destructive interference effects are avoided. Applying the same data window as before and once more considering the highenergy frequency range of the source pulse $(30-80 \mathrm{~Hz})$, MEVA yields the result shown in Fig. VI-10d. It is seen that the maximum value of $v_{\omega}(x)$ is found for the same velocity as in the stacked data case, but now for frequencies where 
MEVA failed before. Hence, it can be concluded that stacking causes low-pass filtering of diffraction energy indeed.

\section{Conclusions:}

(1) For stacked data, MEVA forms a tool for estimating migration velocities.

(2) MEVA can be applied to limited parts of the inverted data set, determining optimal migration velocity for each data window separately.

(3) In the space-frequency domain, MEVA processing time can be reduced by considering a sma11 bandwidth only. For stacked data, low frequencies are generally preferred, since, due to the stacking process, the diffraction energy has been low-pass filtered.

\section{VI.4 MEDICAL APPLICATIONS}

\section{VI.4.1 Zero-offset data measured on a phantom}

A 'phantom' is a physical model containing medium properties and discontinuities which are representative for human tissue configurations. The phantom considered here consists of nylon wires and anechoic holes ('cysts') embedded in a graphite doped gel. The geometry is shown in Fig. VI-1la. Zero-offset measurements have been performed on this phantom within an aperture indicated in Fig. VI-11a, during a registration time such that the response of the third

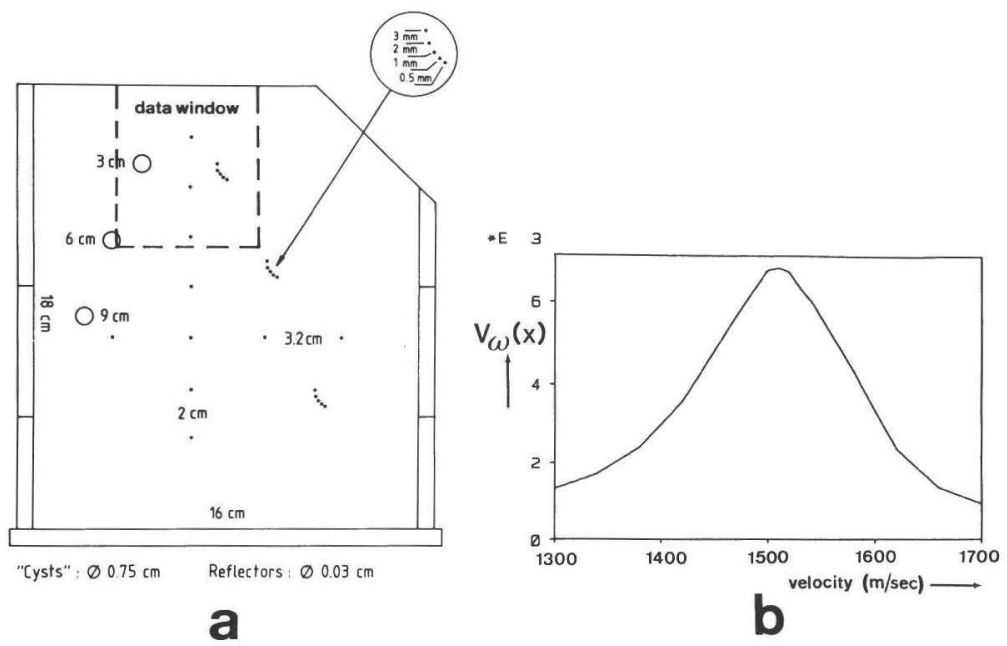

Figure VI-11: MEVA applied to zero-offset data measured on a phantom. a. geometry and data window

b. $v_{\omega}(x)$ as a function of velocity 
wire of the vertical row was just recorded. A transducer was used generating a pulse with a spectrum between 2 and $4 \mathrm{MHz}$.

MEVA was applied to this data set after inversion using all pulse spectrum components, yielding $V_{\omega}(x)$ as a function of velocity as shown in Fig. VI-12b. The maximum value of $V_{\omega}(x)$ is found for velocity value $1505 \mathrm{~m} / \mathrm{s}$, thus being an estimate of the bulk medium velocity. Further experiments showed that MEVA gives consistent results - yielding bulk velocity estimates within a range of $\pm 1 \%$ around $1500 \mathrm{~m} / \mathrm{s}$ - as long as at least one wire response is present in the ME-norm calculation data window. Without such response, the bulk medium with its very dense distribution of graphite particles represents a far too low reflection sparsity to be appropriate for MEVA application.

\section{VI.4.2 Zero-offset data measured on real tissue}

Zero-offset measurements have been performed on a specimen of animal tissue (rumpsteak), fixed in an underwater measurement basin. Recordings were made over an aperture length of $25.6 \mathrm{~mm}$, using a pulse with a spectrum between 1 and $4 \mathrm{MHz}$. Registration time was $0.1 \mathrm{~ms}$. MEVA was applied to the full inverted data set.

Fig. VI-12a shows $V_{\omega}(x)$ as a function of the velocity value used in the nonrecursive inversion process, calculated with consideration of the total pulse bandwidth. A maximum is found for a velocity value $1535 \mathrm{~m} / \mathrm{s}$. Figs. VI-12b,c show that the same results hold if the frequency range considered is reduced to a band around the central frequency, or even to the single central component!

Fig. VI-12d finally shows the image of the specimen.

\section{Concuuㅗㅗㅇㅡ:}

MEVA can successfully be applied to tissue configurations for estimation of bulk velocities and migration velocities.

\section{VI.5 PRACTICAL RECOMMENDATIONS FOR MEVA APPLICATION}

We conclude with the formulation of some practical recommendations for successful MEVA application, based on the discussions in this chapter. 

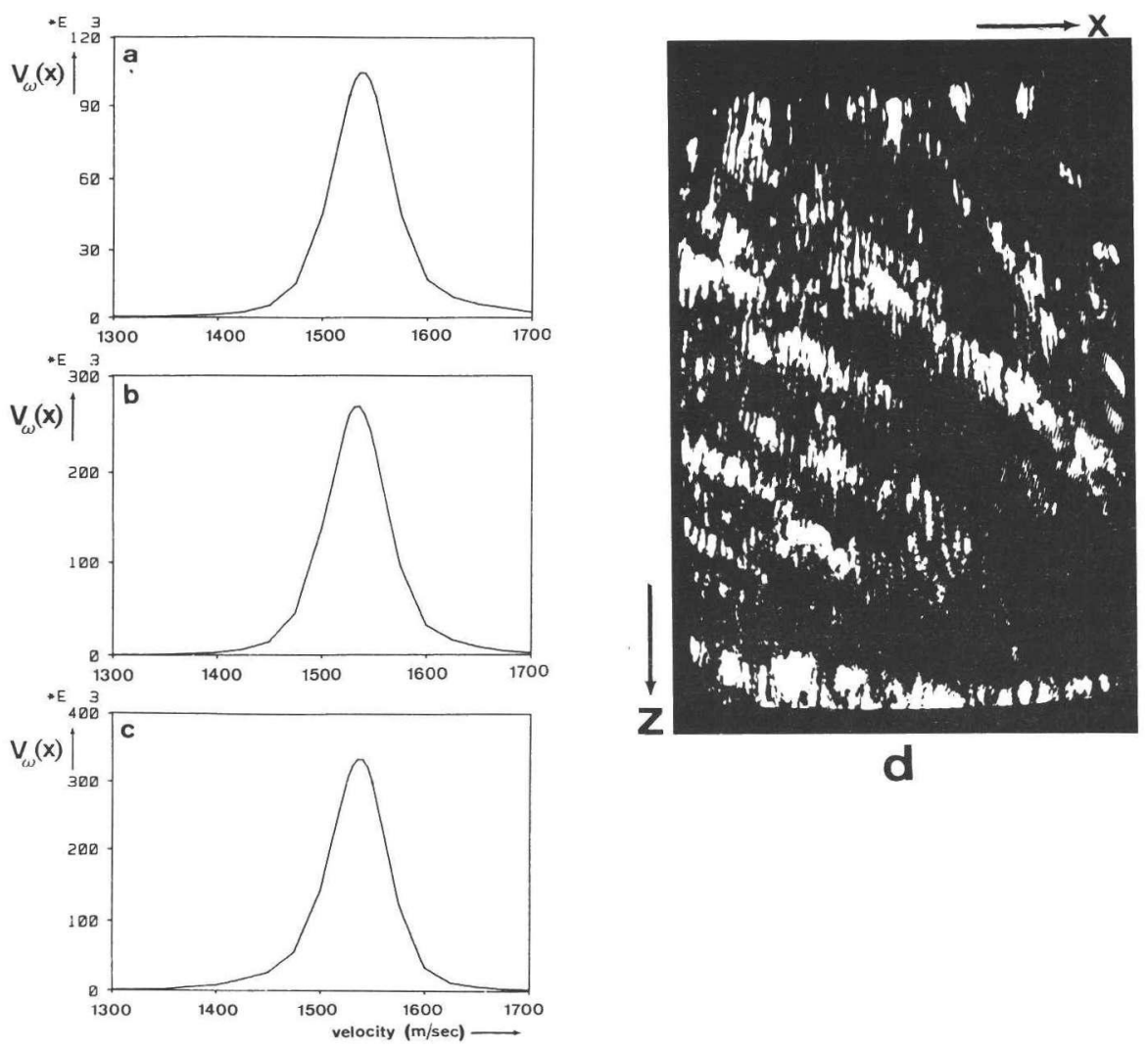

Figure VI-12: MEVA applied to real tissue.
a. broadband result $(1-4 \mathrm{MHz})$
b. result for $2.25-2.75 \mathrm{MHz}$ frequency band
c. result for single frequency component $2.5 \mathrm{MHz}$
d. image of specimen

(1) To obtain a high velocity detectability, entropy function $F(q)$ is appropriately chosen $\mathrm{q}^{4}$ in all MEVA applications.

(2) Since the basic criterion of MEVA is the 1 a $t$ e $r$ a 1 dispersion of an inverted data set, MEVA should principally be applied in the spacefrequency domain or the space-time domain along the $\mathrm{s} p$ a $\mathrm{c}$ e axis. Generally, in the space-frequency domain a higher velocity detectability is obtained. However, MEVA performed in the space-time domain is more attractive from an efficiency point of view. In addition, if the medium under consideration contains low-sparsity diffractor distributions extending in 
both lateral and vertical directions, MEVA is $\mathrm{p} \mathrm{r}$ e $\mathrm{f}$ e $\mathrm{r}$ a b 1 y applied in the space-time domain where, in such cases, the data can be used without transposition as we11 (i.e. MEVA application along the $t \mathrm{im} e$ axis is possible).

(3) If MEVA is applied to zero-offset or stacked data, an estimate of the optimal $\mathrm{m} i \mathrm{~g} r$ a $\mathrm{t}$ o $\mathrm{n}$ velocity is obtained. Local variations of optimal migration velocity can be determined by wi n d o w i n g the data a $f \mathrm{t} e \mathrm{r}$ inversion.

(4) If MEVA is applied to CMP data, an estimate of $\mathrm{s} t$ a $\mathrm{c} \mathrm{k} i \mathrm{n} \mathrm{g}$ velocities is obtained. To obtain the stacking velocity estimate for one specific boundary response, MEVA should be applied within a $t i \mathrm{~m} e \mathrm{w} i \mathrm{n} d \mathrm{o} w$ of the inverted data around the related zero-offset time.

(5) If MEVA is applied to zero-offset or stacked data from 1 a y e $r$ e d media, it is advantageous to include a preprocessing step eliminating a large amount of reflection energy. The result will resemble the response of a two-dimensional diffractor distribution to which MEVA can be applied in the space-time domain as well.

(6) If MEVA is applied in the space-frequency domain, processing time can be reduced by considering only a limited number of spectral components with high signal-to-noise ratio. The contributions of these spectral components may in practice be added without weighting. For stacked data, low-frequency components should be chosen, since stacking introduces low-pass filtering of diffraction energy. 


\section{APPENDIX A:}

\section{A note on the effect of velocity errors in computerized acoustic focusing techniques}

D. de Vries and A. J. Berkhout

Delft University of Technology, Department of Apy lied Physics, Group of Acoustics, P. O. Box 5046, $2600 \mathrm{GA}$ Delft, The Netherlands

(Received 12 April 1982; accepted for publication 22 March 1983)

In computerized acoustic focusing procedures, the values of the propagation velocities of the media between (secondary) sources and registration area must be inserted into the wave-field extrapolation operator. These values are often only approximately known. In this discussion, the effect of velocity errors will be evaluated for several acoustic focusing techniques, active as well as passive.

PACS numbers: 43.60.Gk, 43.85.Ta

\section{INTRODUCTION}

In computerized acoustic focusing procedures, recorded wave-field data are processed with an extrapolation operator (see e.g., Refs. 1 and 2). The authors have shown earlier ${ }^{3}$ that for optimal focusing in terms of axial and lateral resolution, an operator should be applied derived from acoustic wave theory, performing phase correction and amplitude weighting in the space-frequency domain. When correct velocity values are used, the result is a spatially bandlimited, zero-phase image. In the space-time domain, phase correction is replaced by delay correction, so that unweighted focusing can also be described as a procedure compensating for travel times between source and receivers. If the propagation velocities between source and receivers are not accurately known and erroneous values are inserted into the extrapolation operator, the position and the resolution of the focused results are affected in an unfavorable way, as will be shown below.

\section{THEORY}

We consider a point source in an absorption-free, homogeneous medium (velocity $c$ ) with coordinates $(0,0, z)$ in a Cartesian system. The travel times to a recording aperture in plane $z=0$ are given by

$$
\tau(\rho)=\left[(z / c)^{2}+(\rho / c)^{2}\right]^{1 / 2} ; \quad \rho^{2}=x^{2}+y^{2} .
$$

This expression describes a hyperbola in the $\rho-t$ plane, shown as the solid curve in Fig. 1. In paraxial approximation, i.e., $\rho \ll z$, Eq. (1a) may be approximated by a parabolic expression:

$$
\tau(\rho) \approx z / c+\rho^{2} / 2 c z
$$

For optimal focusing of the recorded data, i.e., for reconstruction of an image of the point source with correct position and optimal resolution, the travel times given by Eqs. $(1 \mathrm{a}, \mathrm{b})$ must be correctly compensated for all $\rho$. In general, however, either $z$ or $c$ or both are not known and for a given estimate the time corrections read, in paraxial approximation:

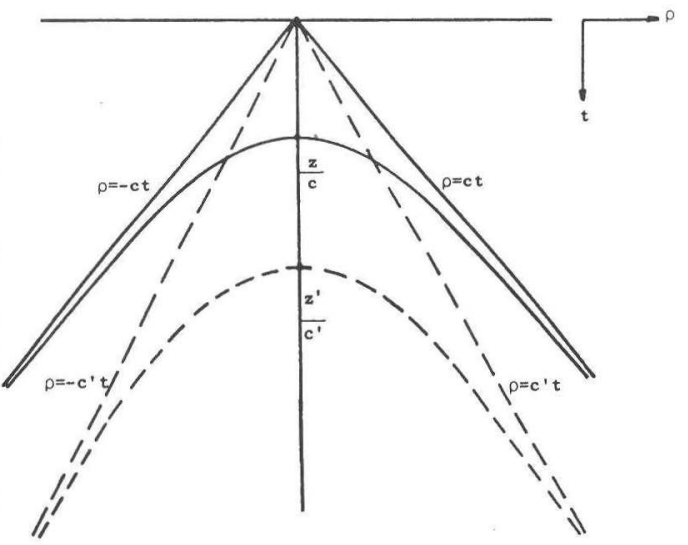

FIG. 1. Travel times and travel-time corrections: two hyperbolae. Solid curve: travel times $\tau(0)$; Dotted curve: travel-time corrections $\tau^{\prime}(\rho)$. 


$$
\tau^{\prime}(\rho) \approx z^{\prime} / c^{\prime}+\rho^{2} / 2 c^{\prime} z^{\prime},
$$

see the dashed curve in Fig. 1. Hence, in general a raypathdependent fraction of the travel times remains uncompensated:

$$
\tau(\rho)-\tau^{\prime}(\rho)=\left(\frac{z}{c}-\frac{z^{\prime}}{c^{\prime}}\right)+\frac{\rho^{2}}{2}\left(\frac{1}{c z}-\frac{1}{c^{\prime} z^{\prime}}\right) .
$$

It is seen from Eq. (3) that, for $c^{\prime} \neq c$, no value of $z^{\prime}$ can be found such that $\tau(\rho)-\tau^{\prime}(\rho)=0$ for all $\rho$, and vice versa.

Now, assuming that $c^{\prime}$ is the available-however wrong - estimate of $c$, we can vary our focusing depth $z^{\prime}$ to optimize our result. We shall discuss two different choices.

\section{(a) Assume $z^{\prime}$ is chosen such that}

$$
z^{\prime}=\left(c^{\prime} / c\right) z
$$

Now, the first term in Eq. (3) vanishes, which means that the vertical travel time $\tau(0)$ is correctly compensated, but a raypath-dependent fraction $\Delta \tau(\rho)$ of all other travel times remains uncompensated:

$$
\Delta \tau(\rho)=\frac{\rho^{2}}{2 c^{2}}\left[1-\left(\frac{c}{c^{\prime}}\right)^{2}\right] \frac{1}{\tau(0)} .
$$

The resulting distortion in the image depends on velocity
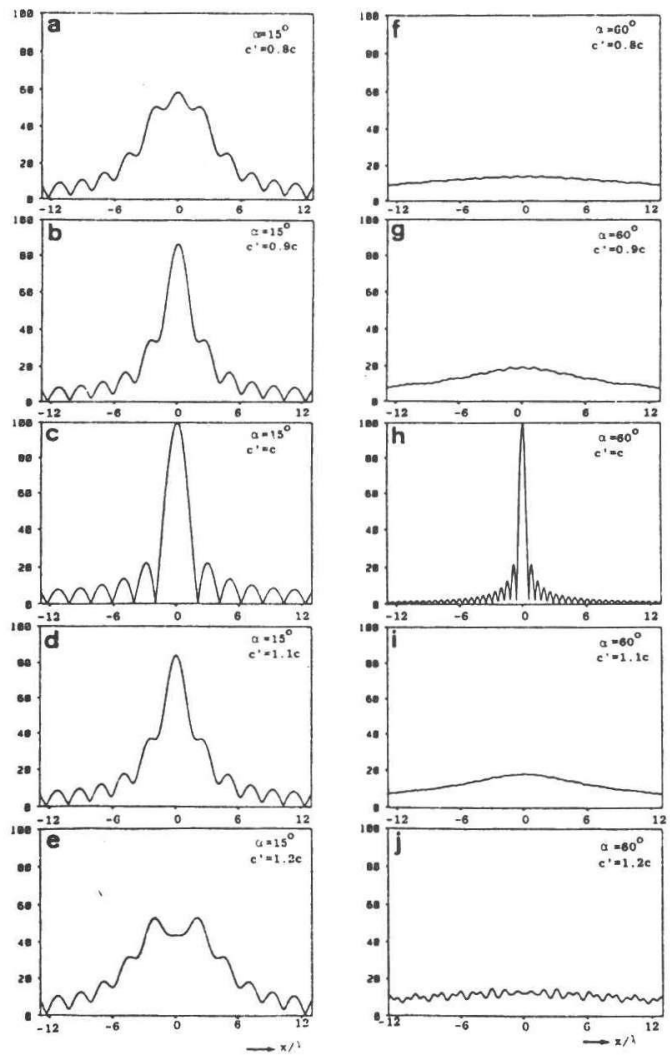

FIG. 2. Focused image of a harmonic dipole line source, when focusing depth $z^{\prime}$ is chosen according to Eq. (4). (a)-(e) give results for an aperture angle of $15^{\circ}$ and various relative velocity errors, (f)-(j) for an angle of $60^{\circ}$. error and aperture angle. In Fig. 2, simulation results are given for a dipole line source generating a harmonic field. It is seen that, especially for large aperture angles, relatively small velocity errors destroy the focused image completely for this choice of focusing depth.

(b) Assume $z^{\prime}$ is chosen such that

$$
z^{\prime}=\left(c / c^{\prime}\right) z
$$

In this case, the second term in Eq. (3) vanishes. This means that after correction a raypath-independent fraction of the travel times remains uncompensated:

$$
\Delta \tau=\left[\left(c^{\prime} / c\right)^{2}-1\right] z^{\prime} / c^{\prime} .
$$

Thus, in the paraxial region to which the validity of Eq. (3) is restricted, all recorded signals are "lined up" after time correction and summation in an appropriate time-window yields maximum output. This means that the focused image has optimal lateral resolution for all velocity errors but it is positioned at a wrong depth given by Eq. (6). This is illustrated in Fig. 3, which also shows that even for large aperture angles where the paraxial approximation does not hold, the
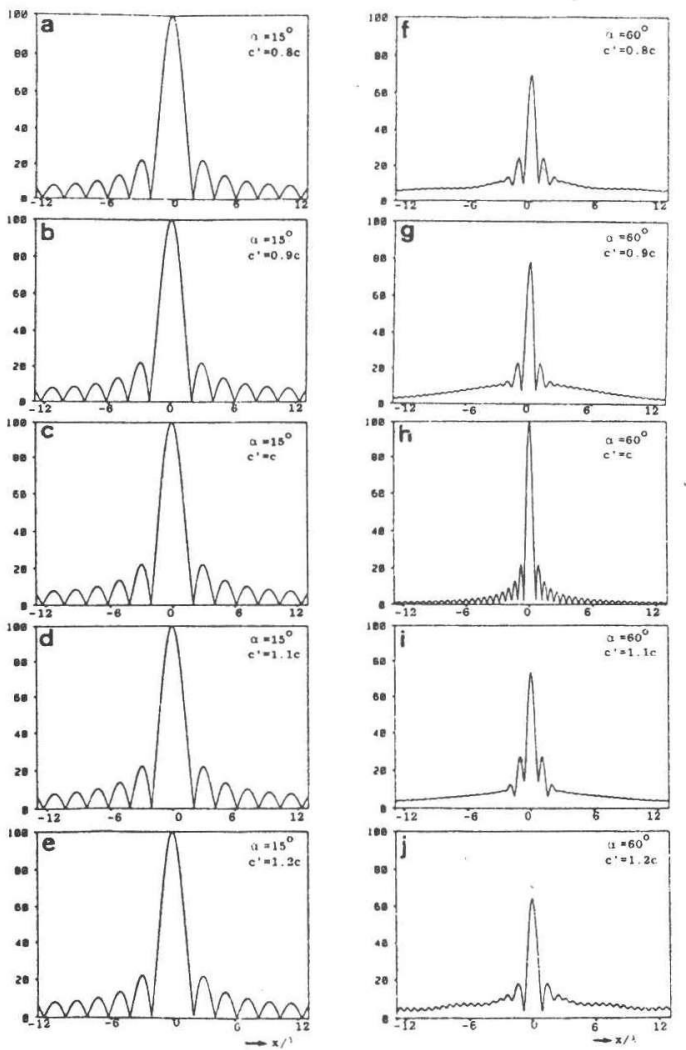

FIG. 3. Focused image of a harmonic dipole line source, when focusing depth $z^{\prime}$ is chosen according to Eq. (6). Parameters as in Fig. 2. 
resolution of the image is hardly affected by velocity errors for this choice of focusing depth.

\section{APPLICATIONS}

In this section we shall apply the above theory to four acoustic imaging systems:

(a) Active acoustic imaging systems based on pulse-echo techniques: (1) using short pulses (high-resolution registration); (2) using lengthy pulses (low-resolution registration).

(b) Passive acoustic imaging systems: (1) detection of impulsive sources; (2) detection of continuous sources.

In this discussion, three time variables should be discriminated:

- travel time, $\tau(\rho)$ :

the time interval between signal generation at the (secondary) point source and signal arrival at the detector with aperture coordinate $\rho$. If the source is situis.ed on the $z$ axis, $\tau(0)$ is called vertical travel time.

- registration time, $T(\rho)$ :

the time interval between start of the recording and signal arrival at the detector.

- travel time correction $\tau^{\prime}(\rho)$ :

correction applied to travel time $\tau(\rho)$ in order to image (focus) the recorded data.

\section{A. High-resolution pulse-echo techniques}

In these techniques, recording is started at the moment that a source on the surface sends a short pulse into the underlaying medium. Diffracted pulses are recorded in the aperture area with high temporal resolution. If sources and detectors are placed in zero-offset or common midpoint configuration, the one-way travel times $\tau(\rho)$ from diffractor to detector can be accurately determined from registration times $T(\rho)$ :

$$
\tau(\rho)=T(\rho) / 2
$$

and can be displayed as a hyperbola in the $\rho-t$ domain with the vertical travel time

$$
\tau(0)=z / c
$$

as apex (see Fig. 4). In these techniques, imaging includes correction to the travel times according to a hyperbola with

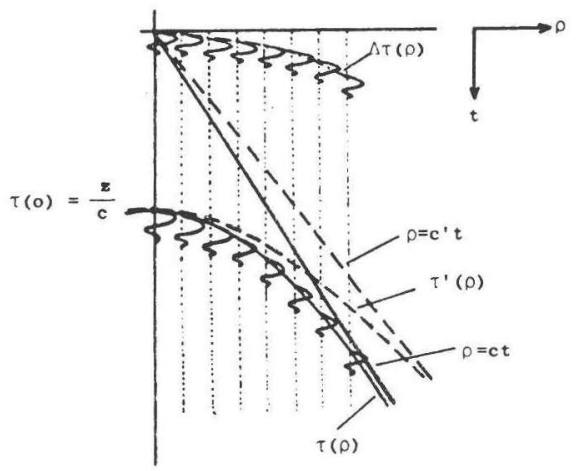

FIG. 4. Imaging procedure in pulse-echo techniques assuming short pulses.

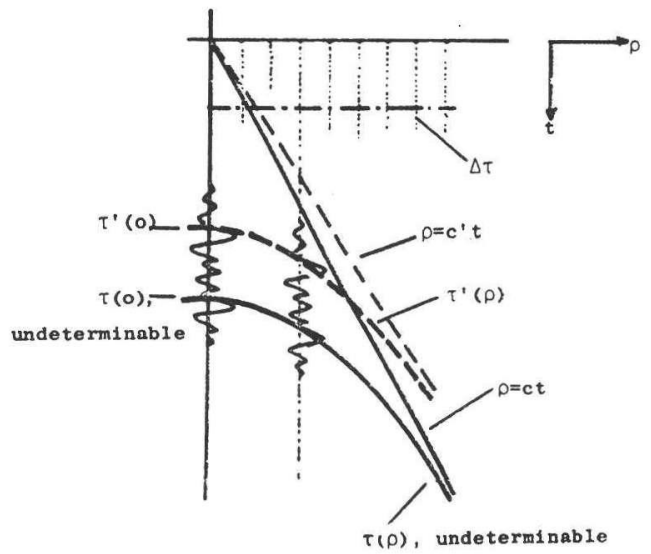

FIG. 5. Imaging procedure in pulse-echo techniques assuming lengthy pulses.

apex $\tau(0)$ which corresponds to choosing focusing depth $z$ according to Eq. (4).

Consequently, the vertical travel time $\tau(0)$ is fully corrected and a raypath-dependent travel time fraction $\Delta \tau(\rho)$, given by Eq. (5), remains uncompensated for $\rho \neq 0$. Full focusing, i.e., maximum output amplitude and correct source depth localization, only occurs when $c^{\prime}=c$. Thus this procedure can be used for velocity determination by varying $c^{\prime}$ and using the output amplitude of the imaged result as a criterion to be maximized.

\section{B. Low-resolution pulse-echo techniques}

As in the previous case, recording is started when a pulse is generated into the medium, but now, due to source properties, absorption, etc., the received pulses have a lengthy character, causing a low temporal resolution in the recorded data. Hence travel times $\tau(\rho)$ cannot be accurately determined from registration times $T(\rho)$. Especially vertical travel time $\tau(0)$ is not well defined (see Fig. 5).

Therefore, the apex $\tau^{\prime}(0)$ of the hyperbola describing the travel-time corrections to be applied for imaging, can be arbitrarily chosen within the length of the recorded signal.

The imaged result is now localized at depth

$z^{\prime}=c^{\prime} \tau^{\prime}(0)$.

By varying $\tau^{\prime}(0)$, the amplitude of the imaged result can be maximized. If $c^{\prime}=c$, this leads to $\tau^{\prime}(0)=\tau(0)$, i.e., correct source localization and full focusing. If, however, $c^{\prime} \neq c$ the maximum amplitude is found for

$$
\tau^{\prime}(0)=\left(c / c^{\prime}\right)^{2} \tau(0),
$$

which corresponds with choosing focusing depth $z^{\prime}$ corresponding to Eq. (6):

$$
z^{\prime}=\left(c / c^{\prime}\right) z \text {. }
$$

In other words: velocity errors are exchanged for vertical travel-time errors and depth errors.

Figure 5 illustrates this situation. Within the paraxial region the travel-time correction hyperbola $\tau^{\prime}(\rho)$ has the same shape as the (unknown) travel-time hyperbola $\tau(\rho)$. 
After time correction all recorded signals are "lined up." A raypath-independent travel-time fraction $\Delta \tau$, given by $\mathrm{Eq}$. (7), remains uncompensated. Note that in this situation an accurate estimate of the medium velocity can not be determined from the imaged result.

\section{Passive imaging in search of impulsive sources (crack detection)}

In these "wait and listen" techniques, recording is started at an arbitrary moment. If in the underlaying medium a "crack" arises, this leads to registration of usually short pulses in the aperture area. As under Sec. IIA, registration times $T(\rho)$ form a well-defined hyperbola, but obviously $T(0)$ has no relation with vertical travel time $\tau(0)$. This means that $\tau(0)$ can by no means be determined.

Therefore we introduce

$$
\theta(\rho)=\tau(\rho)-\tau(0)=\rho^{2} / 2 c z \text {. }
$$

Imaging is now performed by applying a time correction

$$
\theta^{\prime}(\rho)=\rho^{2} / 2 c^{\prime} z^{\prime} \text {. }
$$

By choosing $z^{\prime}$ according to Eq. (6):

$$
z^{\prime}=\left(c / c^{\prime}\right) z,
$$

full-time correction is applied to every raypath and full focusing takes place, but the depth on which the crack is localized is only correct if $c^{\prime}=c$. In conclusion, velocity errors are exchanged for depth errors according to Eq. (14).

\section{Passive imaging in search of continuous sources (e.g., gas leakage detection)}

In this technique, the source may already be active at the moment the recording is started. Thus, as in the previous case, there is no relation between registration time $T(0)$ and vertical travel time $\tau(0)$. The situation is similar to the one discussed under Sec. IIB (see also Fig. 5), except that now the choice of vertical travel-time correction $\tau^{\prime}(0)$ is absolutely free. Again, the image amplitude is maximized by choosing $z^{\prime}$ according to Eq. (6). Correct source localization is only possible if $c^{\prime}=c$, since also in this technique exchange takes place between velocity and depth errors.

\section{CONCLUSION}

It has been shown that the effect of velocity errors on the result of (computerized) acoustic focusing techniques may be quite large, and depends strongly on the application and the optimization technique being used.

'J. F. Claerbout, Fundamentals of Geophysical Data Processing (McGrawHill, New York, 1976).

${ }^{2}$ R. H. Stolt, Geophysics 43 (1), $23-48$ (1978).

${ }^{3} \mathrm{D}$. de Vries and A. J. Berkhout, "Wave theoretical approach to acoustic focusing," J. Acoust. Soc. Am. 70, 740-748 (1981). 


\section{APPENDIX B:}

\section{MAXIMUM ENTROPY SPECTRAL ANALYSIS}

In chapter IV we discussed $m$ i $\mathrm{n} i \mathrm{~m} \mathrm{u}$ m entropy norms to be used as measures of resolving power of a data set. Obviously related, and more current in literature about data analysis, is the concept of $\mathrm{m} \mathrm{a} \mathrm{x} \mathrm{i} m \mathrm{um}$ entropy, commonly used in the context of syectral analysis techniques. This maximum entropy concept, however, concerns $\mathrm{p} r$ o b a b i l i t y d e n s i t y distributions of stochastic data and $n$ o $t$ amplitude distributions.

Hence, it has very little to do with the minimum entropy norms we introduced $^{1)}$, but is directly related to Shannon's entropy, reading in discrete form:

$$
\mathrm{H}=-\sum_{i} \mathrm{p}_{i} \log \mathrm{p}_{i}
$$

with

$$
\sum_{i} p_{i}=1
$$

$p_{i}$ being the probability of event $i$. Merely in order to make the discussion on entropy more complete, we shall now briefly explain the principles of Maximum Entropy Spectral Analysis (MESA) as introduced by Burg in 1967 and applied and refined by himself and many others.

We consider a stationary, stochastic signal $y(t)$, characterized by some probability density distribution. A finite and discretely sampled registration of $y(t)$ is available, from which the discretized autocorrelation function $R_{m}=$ $\mathrm{R}(\mathrm{m} \Delta \tau)$ has been calculated for a limited range of m-values: $|\mathrm{m}| \leqq \mathrm{M}$. Now, an estimation of the powe r s p e c t r u m $S(f)$ of $y(t)$,

1) The concept of minimum-cross-entropy introduced by Shore (1981) is not related to our minimum entropy norms, but is a variant on maximum entropy, used for estimation of spectra of multiple signals. 
which is related to the autocorrelation function $R_{m}$ through discrete Fourier transformation:

$$
S(f)=\Delta \tau \sum_{m=-\infty}^{\infty} R_{m} \exp [-j 2 \pi f m \Delta \tau]
$$

has to be made from the restricted number $(2 M+1)$ of known values of $R_{m}$.

In many spectral analysis techniques, the unknown values of $R_{m}$ are assumed to be zero, which in fact a is very harsh assumption. Therefore, Burg (1967) proposed to find a spectral estimate $\langle S(f)>$ which is consistent with the known values of $R_{m}$, making as few assumptions as possible about the unknown values. This can be realized by requiring that $\langle S(f)\rangle$, with the constraint of consistency with the known values of $R_{m}$, corresponds to the most random signal $y(t)$ and hence is $\mathrm{maximal} 1 \mathrm{y} w \mathrm{~h} i \mathrm{e}$. It was already proven by Shannon (1948) that, for a stationary stochastic signal with given probability density distribution, the entropy $\mathrm{H}$ is maximum if the signal spectrum is white. Hence, the spectral estimate $\langle\mathrm{S}(\mathrm{f})\rangle$ as proposed above is found by $\mathrm{m}$ a $\mathrm{x}$ i m $\mathrm{i}$ z i n g - under the constraint of consistency with the known $R_{m}$-values - the e $n t r o p$ of the signal.

In many discussions about MESA, the stochastic signals considered are assumed to be bandimited $(-\mathrm{B} \leqq \mathrm{f} \leqq \mathrm{B})$, and have a $\mathrm{g}$ a u s s i a $\mathrm{n}$ distribution with an expectation equal to zero, which in practice often (but not always) is approximately true.

For gaussian processes with zero mean it can be derived - see e.g. Haykin and Kessler (1979) - that entropy $H$ is simply related to the autocorrelation m a $\mathrm{t} r \mathrm{i} \times \mathbf{R}$ of the process:

$$
\mathrm{H}=\frac{1}{2} \ln [\operatorname{det} \mathbf{R}] \text {. }
$$

The above authors suggest that, for reasons of convergence, it is more appropriate to introduce in this context the entropy $r$ a $t e h$ :

$$
\begin{aligned}
h & =\frac{1}{2} \ln [\operatorname{det} \mathbf{R}]^{\frac{1}{M+1}} \\
& =\lim _{M \rightarrow \infty} \frac{H}{M+1} .
\end{aligned}
$$


Note that, since $R_{m}=R_{-m}$, $M+1$ denotes the number of $d i f f$ e $\mathrm{e} \mathrm{n} t$ values of the autocorrelation function in the range considered. Haykin and Kessler (1979) also derive a relation, based on Szegö's theorem, between autocorrelation matrix $\mathbf{R}$ and power spectrum $\mathrm{S}(\mathrm{f})$ :

$$
\lim _{M \rightarrow \infty}[\operatorname{det} \mathbf{R}]^{\frac{1}{M+1}}=2 \operatorname{Bexp}\left\{\frac{1}{2 B} \int_{-B}^{B} \ln [S(f)] d f\right\},
$$

where $2 \mathrm{~B}$ denotes the limited signal bandwidth.

Substituting Eq. $(\mathrm{B}-5)$ in $\mathrm{Eq} \cdot(\mathrm{B}-\zeta)$ we find a relation between entropy rate $\mathrm{h}$ and power spectrum $S(f)$ :

$$
h=\frac{1}{2} \ln [2 B]+\frac{1}{4 B} \int_{-B}^{B} \ln [S(f)] d f
$$

Using the relation between power spectrum and autocorrelation function given in Eq. (B-2), and assuming that sampling is done at the Nyquist rate such that

$$
\Delta \tau=\frac{1}{2 B}
$$

we rewrite $\mathrm{Eq} \cdot(\mathrm{B}-6)$ as

$$
h=\frac{1}{4 B} \int_{-B}^{B} \ln \left[\sum_{m=-\infty}^{\infty} R_{m} \exp (-j 2 \pi f m \Delta \tau)\right] d f,
$$

thus giving a relation between entropy rate and the values of $\mathrm{R}_{\mathrm{m}}$ which are known for $|\mathrm{m}| \leqq M$ and unknown for $|\mathrm{m}| \geqq M+1$.

MESA provides an estimate of power spectrum $S(f)$ by maximizing its whiteness and, hence, the corresponding entropy rate within the constraint of consistency with the known values of $R_{m}$. This is done by postulating:

$$
\frac{\partial \mathrm{h}}{\partial \mathrm{R}_{\mathrm{m}}}=0, \quad|\mathrm{~m}| \geqq M+1
$$


By working out Eqs. $(B-8,9)$, as is done quite instructively by Haykin and Kessler (1979), the following expression is found for the maximum entropy power spectrum estimate $\langle\mathrm{S}(\mathrm{f})\rangle$ :

$$
\langle\mathrm{S}(\mathrm{f})\rangle=\frac{\mathrm{P}_{M}}{2 B\left|1+\sum_{\mathrm{m}=1}^{M} a_{m} \exp (-2 j \pi f \mathrm{~m} \Delta \tau)\right|^{2}},
$$

where $\mathrm{P}_{\mathrm{M}}$ is the output of a prediction-error filter of order $\mathrm{M}$ and $\mathrm{a}_{\mathrm{m}}$ are the corresponding filter coefficients chosen in consistence with the known values $R_{m}$ of the autocorrelation function.

Here, as in the definition of resolving power measures, reference to 'entropy' could be omitted: Berkhout (1970) indicated the analogy with prediction error filtering and Van den Bos (1971) proved the equivalence with least-squares fitting of an al1-pole autoregressive model.

Finally it should be mentioned that also in information theory some authors prefer to avoid the word 'entropy', denoting Shannon's measure and related norms as 'information measures' (e.g. Boekee and Van der Lubbe, 1980). 


\section{REFERENCES}

AKI, K. and RICHARDS, F.G. (1980), Quantative seismology. Theory and methods, vol. II, W.H. Freeman \& Co., Allen Cox ed., San Francisco, par. 12.1.

BERKHOUT, A.J. (1970), Minimum phase in sampled-signal theory, thesis, Delft University of Technology, The Netherlands.

BERKHOUT, A.J. (1974), "Related properties of minimum-phase and zero-phase time functions", Geophys. Prosp. vo1. 22, $683-709$.

BERKHOUT, A.J. (1980), Seismic migration. Imaging of acoustic energy by wave field extrapolation. Elsevier Sc. Publ. Cy., Amsterdam-New York-Oxford.

BERKHOUT, A.J. (1982), Seismic migration. Imaging of acoustic energy by wave field extrapolation. A. Theoretical Aspects. 2nd ed. E1sevier Sc. Publ. Cy., Amsterdam-New York-Oxford.

BERRYHILL, J.R. (1979), "Wave-equation datuming", Geophysics vol. 44, $1329-1339$.

BLACKBURN, G. (1980), "Errors in stacking velocity - true velocity conversion over complex geologic situations", Geophysics vol. 45, 1465 - 1488.

BLEISTEIN, N. and COHEN, J.K. (1982), "The velocity inversion problem - Present status, new directions", Geophysics vo1. 47, 1497 - 1511.

BOEKEE, D.E. and VAN DER LUBBE, J.C.A. (1980), "The R-norm information measure", Information and Control vol. 45, 136 - 155.

BOLtZMANN, L. (1872), "Weitere Studien uiber das Wärmegleichgewicht unter Gasmolekulen", Wiener Sitzungsberichte vol. 66, 275 sqq. 
BRILLOUIN, L. (1953), "The negentropy principle of information", J. App1. Phys. vo1. $24,1152-1163$.

BROWN, R.J.S. (1969), "Normal-moveout and velocity relations for flat and dipping beds for long offset", Geophysics vol. 34, 180 - 195.

BURG, J.P. (1967), "Maximum Entropy Spectral Analysis", paper pres. at $37_{\text {th }}$ ann. meeting SEG, Oklahoma City.

CLAERBOUT, J.F. (1977), "Two methods of deconvolution: power spectrum smoothing and parsimonious deconvolution", Stanford Exploration Project Report no. $14,207-226$.

CLAUSIUS, R. (1875, trans1. to French par FOLIE, F. 1888), Théorie méchanique de 1a chaleur, vol. I, Hector Manceau ed., Mons, chp. 4, 138 - 140.

CLAYTON, R.W. and McMECHAN, G.A. (1981), "Inversion of refraction data by wave field continuation", Geophysics vol. 46, 860 - 868 .

COHEN, J.K. and BLEISTEIN, N. (1979), "Velocity inversion procedure for acoustic waves", Geophysics vol. 44, 1077 - 1087.

COOLEY, W.W. and LOHNES, P.R. (1971), Multivariate data analysis, Wiley, New York, N.Y., $144-149$.

DAMERON, D.H. (1979), "Determination of the acoustic velocity in tissues using an inhomogeneous media model", IEEE transac. on sonics and ultrasonics SU-26, $69-74$.

DAMERON, D.H. (1980), "An inhomogeneous media model for the determination of acoustic parameters in tissues", IEEE transac. on sonics and ultrasonics SU-27, $244-248$.

DEEMING, T.J. (1981), "Deconvolution and reflection coefficient estimation using a generalized minimum entropy principle", paper pres. at. 51st meeting SEG, Los Angeles. 
DE VRIES, D. and BERKHOUT, A.J. (1981), "Wave theoretical approach to acoustic focusing", J. Acoust. Soc. Am. vol. 70, $740-748$.

DE VRIES, D. and BERKHOUT, A.J. (1982), "Minimum Entropy as a tool for velocity analysis", Proc. 52nd ann. meeting SEG, Dallas, 32 - 34.

DE VRIES, D. and BERKHOUT, A.J. (1983), "A note on the effect of velocity errors in computerized acoustic focusing techniques", J. Acoust. Soc. Am. vo1. $74,353-356$.

DE VRIES, D. and BERKHOUT, A.J. (1984), "Influence of velocity errors on the focusing aspects of migration", accepted for publication in Geophys. Prosp.

DIEBOLD, J.B. and STOFFA, P.L. (1981), "The trave1-time equation, tau-p mapping, and inversion of common midpoint data", Geophysics vol. 46, $238-254$.

DIX, C.H. (1955), "Seismic velocities from surface measurements", Geophysics vol. $20,68-86$.

DOHERTY, S.M. and CLAERBOUT, J.F. (1974), "Velocity analysis based on the wave equation", Stanford Exploration Project Report no. 1, 160 - 178.

DOHERTY, S.M. and CLAERBOUT, J.F. (1976), "Structure independent velocity estimation", Geophysics vo1. $41,850-881$.

DOHR, G.P. and STILLER, P.K. (1975), "Migration velocity determination: part II. Applications", Geophysics vol. 40,6 - 16,

DURBAUM, H. (1954), "Zur Bestimmung von Wellengeschwindigkeiten aus Reflexions-seismischen Messungen", Geoph. Prosp. vo1. 2, 151 - 167.

EVERETT, J.E. (1974), "Obtaining interval velocities from stacking velocities when dipping horizons are included", Geoph. Prosp. vol. 22, 122 - 142.

GARDNER, G.H.F., FRENCH, W.S. and MATZUK, T. (1974), "Elements of migration and velocity analysis", Geophysics vol. 39, $811-825$. 
GRAY, S.H. and BLEISTEIN, N. (1980), "One-dimensional velocity inversion for acoustic waves: Numerical results", J. Acoust. Soc. Am. vol. 67, 1141 - 1144 .

GRAY, S.H. (1981a), "One-dimensional velocity inversion for acoustic waves: Numerical results II", J. Acoust. Soc. Am. vol. 69, 31 - 34 .

GRAY, S.H., COHEN, J.K. and BLEISTEIN, N. (1980), "Velocity inversion in a stratified medium with separated source and receiver", J. Acoust. Soc. Am. vol. $68,234-240$.

GRAY, S.H. (1981b), "Velocity inversion in a stratified medium with separated source and receiver II", J. Acoust. Soc. Am. vol. 69, 661 - 664.

HAJNAL, Z. and SEREDA, I.T. (1981), "Maximum uncertainty of interval velocity estimates", Geophysics vo1. 46, 1543 - 1547.

HARLAN, W.S., CLAERBOUT, J.F. and ROCCA. F. (1983), "Extracting velocities from diffractions", Proc. 53rd ann. meeting SEG, Las Vegas, 574 - 577.

HAYKIN, S. and KESLER, S. (1979), "Prediction - error filtering and maximum entropy spectral estimation", in: Nonlinear Methods of spectral analysis, Springer Verlag, S. Haykin ed., Berlin-Heidelberg-New York, chp. 2.

HILLER, D. and ERMERT, H. (1982), "U1trasound computerized tomography using transmission and reflection mode", in: Acoustic Imaging vo1. 12, Plenum Press, Eric A. Ash and C.R. Hill eds., New York - London, 553 - 563.

HUBRAL, P. (1975), "Locating a diffractor below plane layers of constant interval velocity and varying dip", Geoph. Prosp. vol. 23, $313-322$.

HUBRAL, P. (1976), "Interval velocities from surface measurements in the threedimensional plane layer case", Geophysics vo1. 41, 233 - 242.

HUBRAL, P. (1980), "Computation of the normal moveout velocity in 3D laterally inhomogeneous media with curved interfaces", Geoph. Prosp. vo1. 28, $221-239$. 
HUBRAL, P. and KREY, Th. (1980), Interval velocities from seismic reflection time measurements, K.L. Larner ed., Tulsa-Oklahoma (S.E.G.).

JAYNES, E.T. (1957), "Information theory and statistical mechanics", Phys. Rev. vol. $106,620 \mathrm{sqq}$ and vol. $108,171 \mathrm{sqq}$.

KAISER, H.F. (1958), "The varimax criterion for analytic rotation in factor analysis", Psychometrika vo1. 23, 187 - 200.

KAVEH, M., SOUMEKH, M., LU, Z.Q., MUELLER, R.K. and GREENLEAF, J.F. (1982), "Further results on diffraction tomography using Rytov's approximation", in: Acoustic Imaging vo1. 12, Plenum Press, Eric A. Ash and C.R. Hill eds., New York - London, 599 - 608.

KENUE, S.K. and GREENLEAF, J.F. (1982), "Limited Angle Multifrequency diffraction tomography", IEEE transac. on sonics and u1trasonics SU-29, $213-217$.

KHATTRI, K., VIG, P.K. and RAO, N.D.J. (1980), "An optimized technique for determining stacking velocity from seismic reflection data", Geoph. Prosp. vol. $28,825-839$.

KREY, Th. (1976), "Computation of interval velocities from common reflection point move-out times for $n$ layers with arbitrary dips and curvatures in three dimensions when assuming small shot-geophone distances", Geoph. Prosp. vol. 24, $91-111$.

LARNER. K.L. and ROONEY, M. (1973), "Interval velocity computation for plane dipping multilayered media", paper pres. at $42 \mathrm{nd}$ ann. meeting SEG, Anaheim.

LEVIN, F.K. (1971), "Apparent velocity from dipping interface reflections", Geophysics vo1. 36, $510-516$.

LOIGNER, E. (1983), "A linear model for velocity anomalies", Geoph. Prosp. vol. $31,98-118$. 
LYNN, W.S. and CLAERBOUT, J.F. (1982), "Velocity estimation in laterally varying media", Geophysics vol. $47,884-897$.

MAY, B.T. and STRALEY, D.K. (1979), "Higher-order moveout spectra", Geophysics vol. $44,1193-1207$.

MESDAG, P.R., DE VRIES, D. and BERKHOUT, A.J. (1982), "An approach to tissue characterization based on wave theory using a new velocity analysis technique", in: Acoustic Imaging vol. 12, Plenum Press, Eric A. Ash and C.R. Hill eds., New York - London, 479 - 491.

MOL, C.R. (1981), U1trasound velocity tomography and dynamic cardiac geometry, thesis, University of Utrecht, The Netherlands.

OOE, M. and ULRYCH, T.J. (1979), "Minimum entropy deconvolution with an exponential transformation", Geophys. Prosp. vo1. 27, 458 - 473.

OWUSU, K., GARDNER, G.H.F. and MASSELL, W.F. (1983), "Velocity estimates derived from three-dimensional seismic data", Geophysics vol. 48, $1486-1497$.

RAZ, S. (1981a), "Direct reconstruction of velocity and density profiles from scattered field data", Geophysics vol. 46, $832-836$.

RAZ, S. (1981b), "Three-dimensional velocity profile inversion from finiteoffset scattering data", Geophysics vo1. 46, 837 - 842 .

ROBINSON, D.E., CHEN, F. and WILSON, L.S. (1982), "Measurement of velocity of propagation for ultrasonic pulse-echo data", U1trasound in Med. \& Biol. vo1. $8,413-420$.

SANZGIRI, S.M. (1977), "Space-time processing of backscattered field for ultrasonic tissue charaterization", Proc. IEEE U1trasonics Symposium, Symposium, $202-205$.

SATTLEGGER, J.W. (1975), "Migration velocity determination: part I. Philosophy", Geophysics vo1. 40, 1 - 5 . 
SATTLEGger, J.W., STiller, P.K. and ECHTERHOFF, J.A. (1976), "Dip-selective migration velocity determination", Geoph. Prosp. vol. 24, 650 - 659.

SAUNDERS, D.R. (1953), "Analytical method for rotation to orthogonal simple structure", Amer. Psychologist vol. 8, 428 (abstract).

SAUNDERS, D.R. (1961), "The rational for an Oblimax method of transformation in factor analysis", Psychometrika vol. 26, 317 - 324.

SChUltZ, P.S. and Claerbout, J.F. (1978), "Velocity estimation and downward continuation by wavefront synthesis", Geophysics vol. 43, $691-714$.

SCHULTZ, P.S. (1982), "A method for direct estimation of interval velocities", Geophysics vol. 47, $1657-1671$.

SHAH, P.M. (1973), "Use of wavefront curvature to relate seismic data with subsurface parameters", Geophysics vo1. 38, 812 - 825.

SHANNON, C.E. (1948), "A mathematical theory of communication", The Bell system Technical Journal vol. 27, $379-623$.

SHORE, J.E. (1981), "Minimum cross-entropy spectral analysis", IEEE transac. on acoustics, speech and signal processing ASSP-29, 230 - 237.

STOFFA, D.L., DIEBOLD, J.B. and BUHL, P. (1982), "Velocity analysis for wide aperture seismic data", Geoph. Prosp. vol. 30, 25 - 57.

STOLT, R.H. (1978), "Migration by Fourier transform", Geophysics vol. 43, $23-48$.

TANER, M.T. and KOEHLER, F. (1969), "Velocity spectra - digital computer derivation and application of velocity functions", Geophysics vol. 34, $859-881$.

TANER, M.T., COOK, E.E. and NEIDELL, N.S. (1970), "Limitations of the reflection seismic system: Lessons from computer simulations", Geophysics vol. $35,551-573$. 
TRIBUS, M. (1978), "Thirty years of information theory", in: The maximum entropy formalism, MIT Press, R.D. Levine and M. Tribus eds., CambridgeMassachusetts-London, 1 - 14 .

VAN DEN BOS, A. (1971), "Alternative interpretation of maximum entropy spectral analysis", IEEE transac. on information theory IT - 17, $493-494$.

WADE, G., ELliotT, S., KHOGEER, I., FlEShER, G., EISLER, J., MENSA, D., RAMESH, N.S. and HEIDBREDER, G. (1978), "Acoustic echo computer tomography", in: Acoustic Imaging vol. 8, Plenum Press, A.F. Metherel1 ed., New York, 565 - 576 .

WEGLEIN, A.B., BOYSE, W.E. and ANDERSON, J.E. (1981), "Obtaining threedimensional velocity information directly from reflection seismic data: An inverse scattering formalism", Geophysics vol. 46, 1116 - 1120.

WIGGINS, R.A. (1978), "Minimum entropy deconvolution", Geoexploration vol. 16, $21-35$.

YILMAZ, O. and CHAMBERS, R.E. (1980), "Migration velocity analysis by wave field extrapolation", paper pres. at 50th ann. meeting SEG, Houston. 


\section{SUMMARY}

In this thesis, a velocity analysis technique is proposed which, in the first place, has been developed for application to echo-acoustical d i f $\mathrm{f} \mathrm{r}$ a c t $i$ o n data, where most techniques known from literature fail to work successfully. Besides, the methode appears to apply to $r$ e $f 1$ e $c t i o n$ data as well, thus forming a1so an alternative to existing techniques.

The basic idea behind the proposed technique is that an $i \mathrm{n} v$ e $\mathrm{t}$ e $\mathrm{d}$ wave

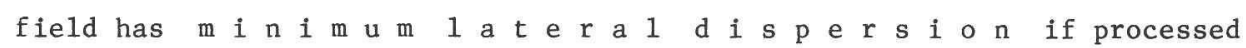
with $c \circ r$ e $t$ ve $1 \circ c i t y$. For a given sparsity of diffractors, lateral dispersion can appropriately be quantified by a norm

$$
\mathrm{v}=\frac{1}{\mathrm{~N}} \sum_{i=1}^{\mathrm{N}} \mathrm{q}_{i} \mathrm{~F}\left(\mathrm{q}_{\mathbf{i}}\right),
$$

where $N$ is the number of data points in lateral direction, $q_{i}$ is a normalized amplitude parameter and $\mathrm{F}\left(\mathrm{q}_{1}\right)$ is a function monotonically increasing with $q_{i}$, thus emphasizing amplitude peaks in the data. Since norms of this type have earlier been used in geophysics, for deconvolution purposes, under the name $m i n i m u$ e $n t r o p y$ norms, this name has been adopted and the

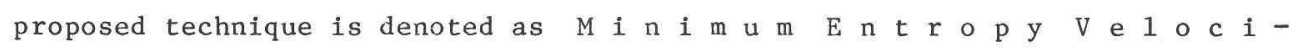
$t$ y A n a 1 y s i s (MEVA).

In chapter I, a survey is given of existing velocity analysis techniques and its applications, leading to the conclusion that, for diffraction data, techniques with general applicability are not available and new ways should be explored.

In chapter II, a wave theoretical model for modeling and inversion of primary compressional waves is discussed. In particular, the model is adapted to $z$ e $r$ o- of f s e $t$ data acquisition, since zero-offset - or, more in general, common offset - techniques are very suitable to extract information from diffraction data. The analogy between zero-offset propagation of $d$ i f $f r a c t i o n$ energy and common midpoint propagation of $r$ e $f 1$ e c $t i \circ n$ energy is illustrated. 
In chapter III, focussing quality of an inversion operator is described in terms of the $s p a t i a l$ wa ve 1 e $t$ obtained a f $t$ e $r$ i n v e r $\mathrm{s} i \mathrm{o}$. This spatial wavelet represents, in the space-frequency domain, the image of an elementary diffractor. It is shown that the 1 a $t$ e $r$ a 1 d i s $\mathrm{p}$ e r s $i$ o $n$ of the inverted spatial wavelet increases if a $v$ e 1 o c $i t y$ e $r \quad r \circ r$ is introduced into the inversion operator. This phenomenon will appear to form the basis of MEVA.

In chapter IV, the e n t r $\circ \mathrm{p} y$ concept is introduced. Shannon proposed entropy as a measure, based on probability density functions of stochastic variables, of a priori $u n c$ e $r t$ a $i n t y$ in communication theory. Maximum entropy corresponds with maximum uncertainty. By changing the sign, a measure of a priori $c$ e $r$ a $i n t y$ can be defined, having maximum value for minimum entropy. It is shown that for a one-dimensional data set (which is not necessarily stochastic), a measure of $r$ e s o $1 \mathrm{v}$ i n g p o w e r can be defined, the form of which is fully identical to the certainty measure mentioned above, except that probability is now replaced by amplitude. Due to the formal analogy, we call this measure a $m i n i m u m$ e $n t r o p y$ (ME) norm. The value of an ME-norm is determined by the sparsity of events (in our applications: echo's) as well as the dispersion of the pulses representing those events. Dispersion, in its turn, is determined by bandwidth and phase spectrum. ME-norms are also defined for a two-dimensional data set in the space-time domain. The sensitivity of ME-norms to variations in elementary data set properties as sparsity, bandwidth, phase spectrum and noise are discussed.

In chapter $\mathrm{V}$, the formal analogy between one echo-acoustical data trace in the time domain and one spectral component of a two-dimensional data set in the space-frequency domain is demonstrated. From this, the conclusion is drawn that all properties derived for ME-norms applied to data in the space-time domain, are also valid for ME-norms applied to data in the space-frequency domain. In the latter case, the role of temporal wavelets (pulses) is performed by $s$ p a $i$ a 1 wavelets. In the space-frequency domain, ME-norms quantify lateral resolving power, for a given sparsity of diffractors determined by 1 a $t$ e $r$ a 1 d $i s$ e r s $i$ o . Since in chapter III it was shown that lateral dispersion is affected by velocity errors, the basic validity of MEVA in the space-frequency domain is thus made clear. It is shown that MEVA can also be applied in the space-time domain, after data transposition, since 
lateral dispersion of spatial wavelets also appears in that domain as lateral extension of diffractor images. As an example, MEVA is applied to the zerooffset response of a single diffractor, which in the present context may also be interpreted as the common midpoint response of a reflector. In this way, principal applicability of MEVA to zero-offset diffraction data as we11 as CMP reflection data is demonstrated.

In chapter VI, applications of MEVA are discussed. Simulated as we11 as measured data are taken into consideration, related to the fields of seismics and medical ultrasound diagnostics. It is concluded that, applied to zero-offset diffraction data, MEVA is a successful tool for the determination of migration velocities. Applied to CMP reflection data, MEVA is an interesting alternative to existing techniques aiming at estimation of stacking velocities. 


\section{SAMENVATTING}

\section{SNELHEIDSANALYSE GEBASEERD OP 'MINIMUM ENTROPY'}

In dit proefschrift wordt een snelheidsanalysetechniek geïntroduceerd die in de eerste plaats werd ontwikkeld voor toepassing op echo-akoestische d i f f $\mathrm{r}$ a c t i e - data. Ontwikkeling van een dergelijke techniek was gewenst, omdat uit de literatuur bekende methoden slechts geschikt zijn voor behandeling van $r$ e f 1 e c t $i$ e-energie. Overigens blijkt de voorgestelde methode ook toepasbaar op reflectie-data, daarmee een alternatief vormend voor de bestaande technieken.

De basisgedachte achter de voorgestelde techniek is, dat een $\mathrm{g}$ e $\mathbf{I} \mathrm{n} v$ e $\mathrm{r}-$ t e e r d golfveld m i n $i$ m 1 e 1 a $t$ e $r$ a 1 e d i s p e r s i e heeft indien de inversie met de $j u$ is $t$ e $s \mathrm{n}$ e $1 \mathrm{~h}$ e d e $n$ is uitgevoerd. Voor een gegeven dichtheid van diffractoren kan laterale dispersie worden gekwantificeerd door een norm

$$
v=\frac{1}{N} \sum_{i=1}^{N} q_{i} F\left(q_{i}\right),
$$

waarin $\mathrm{N}$ het aantal data punten in laterale richting voorstelt, $\mathrm{q}_{1}$ een genormeerde amplitudeparameter is en $F\left(q_{i}\right)$ een functie die monotoon stijgt met argument $q_{i}$. Men ziet gemakkelijk in dat $v$ hoge waarden aanneemt indien de amplitudeverdeling een klein aantal hoge pieken bevat. Normen van dit type werden eerder toegepast in de geofysische signaalverwerking ter deconvolutie van seismische registraties onder de naam " m i n i m u m e $\mathrm{t}$ r o p y " normen. Deze naam is overgenomen en de voorgestelde snelheidsanalysetechniek wordt aangeduid als "Minimum Entropy Velocity Analysis" (MEVA).

In hoofdstuk I wordt een overzicht gegeven van uit de literatuur bekende snelheidsanalysetechnieken en hun toepassingsmogelijkheden. De conclusie wordt getrokken dat, voor diffractie-data, technieken met algemene toepasbaarheid niet beschikbaar zijn zodat naar nieuwe mogelijkheden moet worden gezocht. 
In hoofdstuk II wordt een fysisch model voor het modelleren en inverteren van compressiegolven besproken, gebaseerd op de akoestische golftheorie. Het model wordt speciaal toegespitst op zgn. " z e r o - o f f s e t " - technieken, waar bron en ontvanger zich op dezelfde plaats aan het oppervlak van het onderzochte medium bevinden. Dergelijke technieken zijn zeer geschikt om informatie te verkrijgen uit diffractiedata. De analogie tussen de voortplanting van

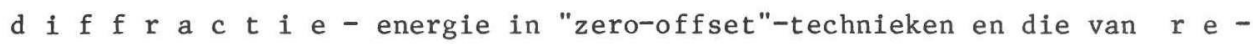
f 1 e c $t i$ e - energie in "common midpoint"-technieken (bron en ontvanger staan steeds evenver aan weerszijden van een gemeenschappelijk middelpunt) wordt aangegeven.

In hoofdstuk III wordt de focusseringskwaliteit van een inversieoperator beschreven met behulp van het "spatial wavelet" dat n a i n v e r s i e resulteert. Dit "spatial wavelet" is een ruimtelijke amplitudeverdeling die, in het ruimte-frequentie-domein, de afbeelding van een elementaire diffractor representeert. Aangetoond wordt dat de 1 a t e r a 1 e d i s p e r s i e van dit "spatial wavelet" toeneemt wanneer in de inversieoperator een snelheidsfout wordt geintroduceerd. Dit verschijnsel zal de basis blijken te vormen voor MEVA.

In hoofdstuk IV wordt het begrip e $\mathrm{n} \mathrm{r} \circ \mathrm{p} i$ e geintroduceerd. Shannon voerde entropie in als een mat voor a priori o $n \mathrm{z}$ e $\mathrm{k}$ r h e $\mathrm{i} d$ in de communicatietheorie. Hierbij is entropie gebaseerd op de waarschijnlijkheidsdichtheidsfuncties van stochastische variabelen. Maximale entropie correspondeert met maximale onzekerheid. Door het teken te veranderen, kan een maat voor a priori $\mathrm{z}$ e $\mathrm{k}$ e $\mathrm{r}$ h e $\mathrm{i} d$ worden gedefinieerd, die een maximale waarde heeft voor minimale entropie. Getoond wordt hoe, voor een één-dimensionale datareeks (die niet stochastisch hoeft te zijn) een mat voor $s c h$ e i $\mathrm{d}$ e $\mathrm{n} d \mathrm{v}$ e $\mathrm{r} \mathrm{m} \circ \mathrm{g}$ e $\mathrm{n}$ kan worden gedefinieerd die qua vorm geheel identiek is aan bovengenoemde zekerheidsmat; alleen is waarschijnlijkheid nu vervangen door amplitude. Vanwege de formele analogie noemen we deze maat een " m i n $\mathrm{i}$ m u m e n t r o p y " (ME)-norm. De waarde van een ME-norm wordt bepaald door de dichtheid van te registreren parameters (in deze toepassingen: echo's) alsmede door bandbreedte en fasespectrum van de tijdpuls die zo'n parameter in beeld brengt. Bandbreedte en fasespectrum bepalen tezamen de dispersie van de puls. Behalve voor een één-dimensionale datareeks worden ME-normen ook gedefinieerd voor twee-dimensionale datasets in het ruimte-tijd-domein. Tenslotte wordt de gevoeligheid van ME-normen besproken voor variaties in eigenschappen van de dataset, zoals parameterdichtheid, bandbreedte, fasespectrum en ruis. 
In hoofdstuk $\mathrm{V}$ wordt de formele analogie aangetoond tussen één echo-akoestische dataregistratie in het tijddomein en één spectrale component van een twee-dimensionale dataset in het ruimte-frequentie-domein. Hieruit wordt de conclusie getrokken dat alle eigenschappen die werden afgeleid voor ME-normen toegepast op data in het ruimte-tijd-domein, principieel ook gelden voor MEnormen toegepast op data in het ruimte-frequentie-domein. In het laatste geval wordt de rol van de tijdpulsen overgenomen door eerdergenoemde "spatial wavelets". In het ruimte-frequentie-domein zijn ME-normen een maat voor lateraal scheidend vermogen. Voor een gegeven diffractordichtheid wordt dit scheidend vermogen bepaald door 1 a $t$ e r a 1 e d i s p e r s i e. Aangezien in hoofdstuk III werd gedemonstreerd dat laterale dispersie wordt beïnvloed door sne1heidsfouten is hiermee de principiële toepasbaarheid van MEVA in het ruimtefrequentie-domein aangetoond. Voorts wordt aangegeven dat MEVA ook kan worden toegepast in het ruimte-tijd-domein via datatranspositie, aangezien laterale dispersie van "spatial wavelets" in dat domein tot uiting komt als laterale verbreding van diffractorafbeeldingen. Als voorbeeld wordt MEVA toegepast op de "zero-offset"-responsie van een enkele diffractor, die in deze context ook mag worden geinterpreteerd als de "common midpoint"-responsie van een reflector. Aldus wordt de principiële toepasbaarheid van MEVA op "zero-offset" diffractie-data zowel als "common offset" reflectie-data aangetoond.

In hoofdstuk VI worden toepassingen van MEVA besproken. Gesimuleerde zowe1 als gemeten data worden beschouwd, betrekking hebbend op de toepassingsgebieden seismiek en medische diagnostiek.

Geconcludeerd wordt dat MEVA met succes kan worden toegepast op "zero-offset" registraties van diffractie-data teneinde migratiesnelheden te bepalen. Toegepast op "common midpoint" reflectie-data vormt MEVA een interessant alternatief voor bestaande methoden om "stack"-snelheden te bepalen. 


\section{CURRICULUM VITAE}

van Diemer de Vries

3 januari 1945 - geboren te Munnekeburen, Friesland, als eerste zoon van Jeen de Vries en Elizabeth Bos

$1950-1956$

- lager onderwijs te Arnhem en Heemstede'

$1956-1963$

1962

1963

- middelbare schoolopleiding aan het Christelijk Lyceum te Haarlem

- eindexamen gyınasium- $\beta$

- staatsexamen gymnasium- $\alpha$

$1963-1971$

$1968-1971$

$1970-1971$

22 juni 1971

- studie voor natuurkundig ingenieur aan de Technische Hogeschool te De1ft

- 4e- en 5e-jaarswerk in de onderzoekgroep Akoestiek op het gebied van mechanische trillingsleer en zaalakoestiek, o.1.v. prof.dr.ir. C.W. Kosten en lector ir.

D.W. van Wulfften Palthe

- student-assistent in de onderzoekgroep Akoestiek

- doctoraal examen natuurkundig ingenieur

$1966-1967$

- praeses van het bestuur van sociëteit "De Koornbeurs" der S.S.R.-Delft

september 1968

- eerste contrabasles;

begin van vele activiteiten in het amateurmuziekleven, met name op het klassieke terrein

1971 - heden

- wetenschappelijk medewerker, resp. hoofdmedewerker in de onderzoekgroep Akoestiek;

research op het gebied der zaal- en bouwakoestiek, sinds 1978 ook op het gebied der echo-akoestiek

3 juli 1974

- getrouwd met Nel van Waert 


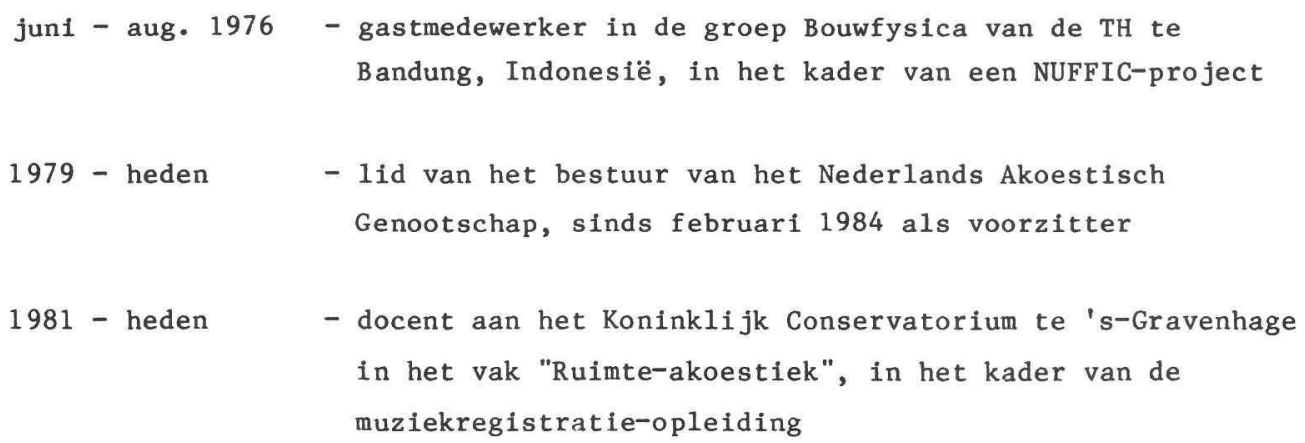

15 september 1982 - geboorte van zoon Tjalling 


8. Medisch onderzoek op consultatiebureau's voor zuigelingen en kleuters dient, in plaats van door huisartsen, te worden verricht door in preventieve benadering geschoolde jeugdartsen.

9. Het oproepen tot stakingen bij openbare diensten dient te worden bestraft als een economisch delict.

10. Het vaderschap leidt tot ingrijpende herziening van opvattingen betreffende wereldvraagstukken.

11. Een promovendus kan met tien stellingen volstaan. 


\author{
STELLINGEN \\ behorende bij het proefschrift \\ "Velocity Analysis based on Minimum Entropy"
}

1. "Minimum Entropy"-normen toegepast op geïnverteerde data in het $(x, \omega)$ domein kunnen, behalve voor snelheidsanalyse, in algemenere zin dienen als mat voor de focusseringskwaliteit van de gebruikte inversieoperator. (Dit proefschrift)

2. De beperkte toepasbaarheid van "Minimum Entropy"-normen voor deconvolutie van seismische data kan simpel worden angetoond door de maximal discrimineerbare reflectiedichtheid te vergelijken met het WT-criterium.

(Dit proefschrift)

3. Bij alle toepassingen van geluid als afbeeldingsmiddel dient men wel te beseffen dat Q́KOVElv "horen" betekent en dat de akoestische wetenschap zich derhalve steeds ook met hoorbaar geluid moet (blijven) bezighouden.

4. In de zaalakoestische adviespraktijk wordt doorgaans te weinig aandacht besteed aan luidheidsaspecten.

5. Aangezien van een lange dunne snaar de boventonen harmonischer zijn dan van een korte dikke snaar met dezelfde grondtoon, verdient toonomvangvergroting van de contrabas tot $C$ door middel van een verlengde E-snaar de voorkeur boven het toevoegen van een vijfde snaar.

6. Trillingen die door celli en contrabassen via de steunpen in een podium worden opgewekt veroorzaken een akoestisch nabijheidsveld dat slechts dient tot muzikale zelfbevrediging van de bespelers.

7. Een overschot aan ingenieurs is eenvoudig te voorkomen door goede beheersing van de Nederlandse taal als voorwaarde te stellen voor het verkrijgen van het ingenieursdiploma. 

Gebotekst Zoetermeer/1984 